\title{
Design and Performance of LoAd BeARING SheAR WALLS MADE FROM Composite Rice Straw Blocks
}

\author{
A Thesis \\ presented to \\ the Faculty of California Polytechnic State University, \\ San Luis Obispo
}

\author{
In Partial Fulfillment \\ of the Requirements for the Degree \\ Master of Science in Civil and Environmental Engineering
}

by

Kevin Robert Camann

December • 2009 
(C) 2009

Kevin Robert Camann

ALL RIGHTS RESERVED 


\section{Committee Membership}

TITLE:

AUTHOR:

DATE SUBMITTED:

COMMITTEE CHAIR:

COMMITTEE MEMBER: Charles Chadwell, Associate Professor

COMMITTEE MEMBER: Bing Qu, Assistant Professor
Design and Performance of Load Bearing Shear Walls

Made from Composite Rice Straw Blocks

Kevin Robert Camann

December 2009

Daniel C. Jansen, Associate Professor 


\section{Abstract \\ Design and Performance of Load Bearing Shear Walls \\ Made from Composite Rice Straw Blocks}

Kevin Robert Camann

Although rice straw and other grains have been used in building since pre-history, in the past two decades, there has been a move to utilize this rapidly renewable, locally available, agricultural byproduct as part of the sustainable construction movement. Up to this point, this has been done by simply stacking up the full straw bales. Stak Block, invented by Oryzatech, Inc., is a modular, interlocking block made of a composite of rice straw and binding agent that serves as an evolution in straw construction. This study investigates the feasibility of using these Stak Blocks as a structural system. The report was divided into four main parts: material testing, development of effective construction detailing, full-scale physical shear wall testing, and a comparison with wood framed shear walls.

The first section investigated the feasibility of using the Stak Blocks in a loadbearing wall application. Constitutive properties of the composite straw material such as yield strength and elastic stiffness were determined and then compared to conventional straw bale. Next, the decision was made to prestress the walls to create a more effective structural system. Various construction detailing iterations were evaluated upon the fullscale shear wall testing using a pseudo-static cyclic loading protocol. Finally, the available ductility of the prestressed Stak Block walls in a lateral force resisting application is quantified along with an approximation of potential design shear forces.

It was determined that the Stak Block material performed satisfactorily in gravity and lateral force resisting applications, in some respects better than conventional woodframed construction, and has great potential as a seismically-resistant building material.

Keywords: Stak Block, Straw Block, Straw Bale Construction, Prestressed Shear Wall, Green Construction, Sustainable Construction 


\section{Acknowledgements}

This project simply could not have taken place without Ben Korman and Jay Ruskey at Oryzatech, Inc. Their foresight and creativity in dreaming up these blocks made of rice straw is great to see in an industry where building codes and familiarity with conventional methods can sometimes stifle new ideas.

I will always remember my time at Cal Poly as an extremely positive one where I grew as an engineer and as a person. I was allowed to explore the passion and interest I always had in the sustainable construction movement while testing and building with unconventional materials. The "Learn by Doing” credo that Cal Poly advertises could not have rung truer to describe my experience over these past two years.

I cannot thank Dr. Dan Jansen enough for coming across this project and immediately thinking of me. Your shared enthusiasm in my thesis was not taken for granted and I immensely appreciated all of the time you helped me whether it was brainstorming construction ideas or setting up different laboratory tests. I would also like to thank Dr. Charles Chadwell, whose experience with the testing and modeling of shear walls was greatly appreciated. The same sentiment goes for Dr. Bing Qu, who provided great insight into the ductility and behavior seen in testing.

I would also like to recognize practicing engineers and architects such as Bruce King who were championing sustainable construction choices before it was the "en vogue" thing to do. Another thank you to fellow Civil Engineering graduate students such as Matt Porter and Horacio Heredia, as well as the many other students whose genuine interest in the project reminded me how "cool” of a thesis topic I ended up with.

My family I would like to thank for their unwavering support of me when I called to say I would be building houses out of straw. Three little pigs' jokes aside, it means so much to have you guys be so proud of what I do.

Last, but certainly not least, I would like to thank my future fiancé Beth. Without your calming presence and ability to keep me on task when I get distracted, this thesis would have literally taken years. For this dedication and unwavering love I will forever be grateful. 


\section{Table of Contents}

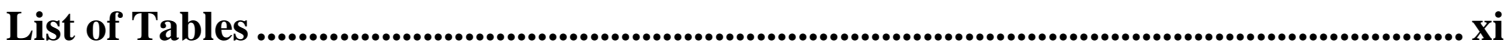

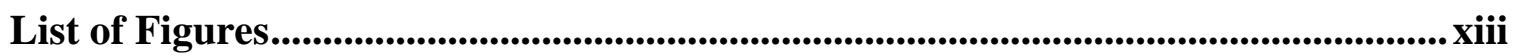

Chapter 1 Introduction .......................................................................................... 1

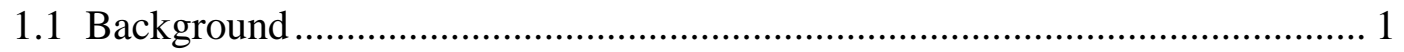

1.1.1 Historical Usage of Rice Straw............................................................... 2

1.1.2 Stak Block Product Manufacturing .......................................................... 2

1.1.3 Potential for Stak Block Production............................................................ 5

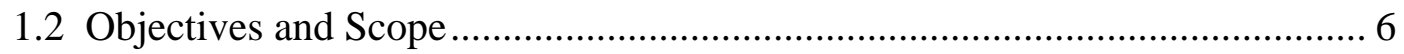

1.3 Organization and Contents......................................................................... 7

Chapter 2 Literature Review........................................................................................ 10

2.1 Straw Bale Construction ................................................................................ 11

2.1.1 Basic Testing of Straw Bales and Straw Bale Wall Systems ...................... 11

2.1.2 Investigations into Plastered Straw Bale Construction ............................... 14

2.1.3 In-Plane Cyclic Testing of Plastered Straw Bale Wall Assemblies ........... 15

2.1.4.1 Fire Testing .......................................................................................... 17

2.1.4.2 Thermal Properties ............................................................................... 17

2.2 Development of Testing Protocol and Model for Analyzing Timber Structures18

Chapter $3 \quad$ Materials Investigation ............................................................................ 21

3.1 Measurement of Straw Block Constitutive Properties....................................... 22

3.1.1 Measurement of Strain ................................................................................ 23

3.1.2 Testing Program Terminology ................................................................ 24

3.1.3 Compression Testing Protocol ................................................................. 27

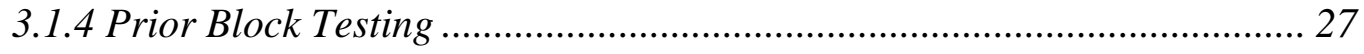


3.1.5 Comparison of Full Blocks to Half Blocks................................................. 28

3.1.6 Relation of Strength to Density ................................................................. 30

3.1.7 Cyclic Testing of Blocks ............................................................................. 34

3.1.8 Preliminary Compression Testing Results ............................................... 41

3.1.9 Relaxation Testing over Time.................................................................... 43

3.1.10 Block Material Properties Post-Shear Wall Testing............................... 46

3.1.11 Comparison to Straw Bale Testing........................................................... 49

3.2 Prestressing Materials Investigation ................................................................. 51

3.2.1 Tensile Testing Setup............................................................................. 52

3.2.2 Threaded Rod Testing ................................................................................ 53

3.2.3 Wire Rope Testing ............................................................................. 57

3.2.4 Prestressing Material Discussion ............................................................. 58

3.2.5 Potential for Bamboo Prestressing ............................................................ 59

3.3 Plywood Fastening Investigation..................................................................... 62

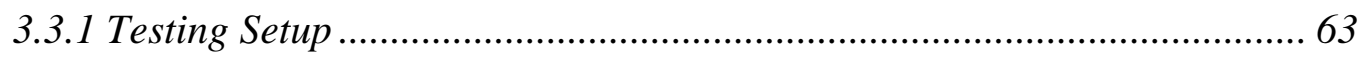

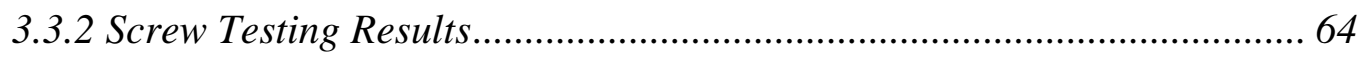

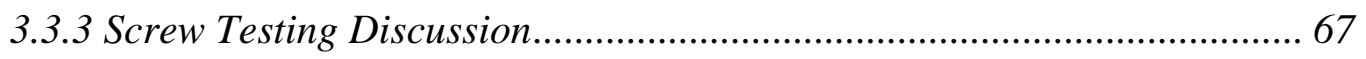

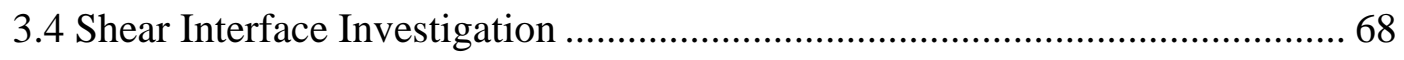

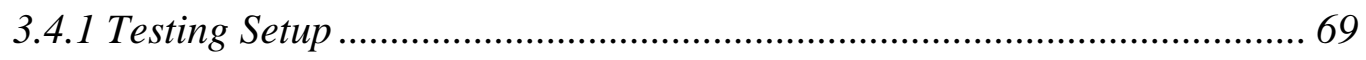

3.4.2 Comparison of Block-Block to Block-Wood Interface Shear Transfer ..... 70

Chapter 4 Testing of Full-Scale Shear Walls ........................................................... 72

4.1 Laboratory Setup for Shear Wall Testing ........................................................ 73

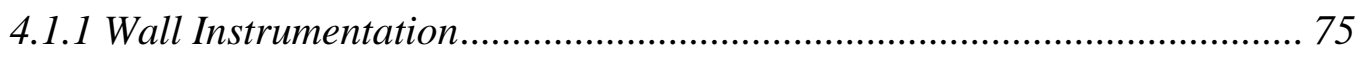

4.2 Full-Scale Shear Wall Testing Protocol............................................................. 77

4.3 Wall Prestressing ......................................................................................... 79

4.3.1 Decision to Prestress.................................................................................. 79

4.3.2 Wall Finite Element Analysis .................................................................... 82 


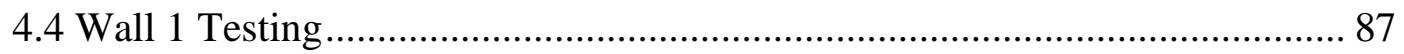

4.4.1 Wall 1 Configuration................................................................................... 87

4.4.2 Wall 1 Testing Results.............................................................................. 88

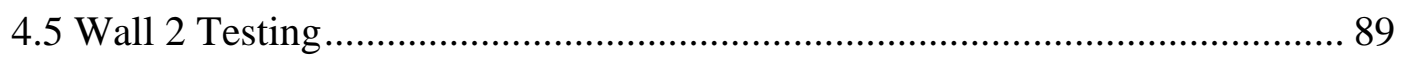

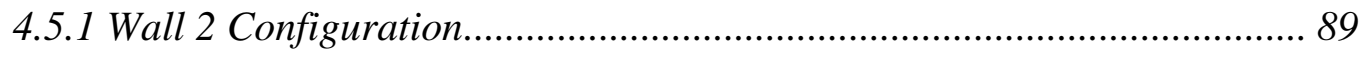

4.5.2 Wall 2 Testing Results........................................................................... 92

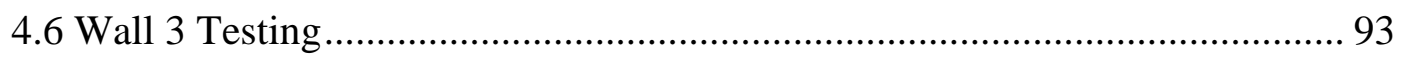

4.6.1 Wall 3 Configuration............................................................................ 93

4.6.2 Wall 3 Testing Results......................................................................... 96

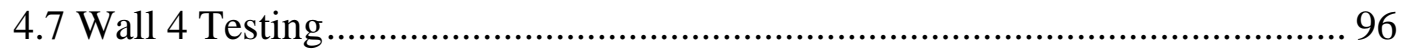

4.7.1 Wall 4 Configuration............................................................................ 96

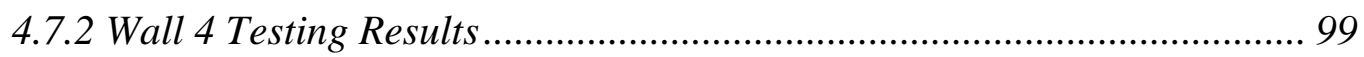

4.8 Summary of Test Results ................................................................................ 99

Chapter 5 Full-Scale Shear Wall Results ..................................................................... 101

5.1 Prestressing Tensile Forces during Testing ..................................................... 101

5.1.1 Prestressed Wall Behavior ..................................................................... 101

5.1.2 Calculation of Block Stresses during Testing ......................................... 104

5.1.3 Forces within Wire Rope versus Thread Bar .......................................... 108

5.1.4 Thread Bar Yielding and Elongation ...................................................... 109

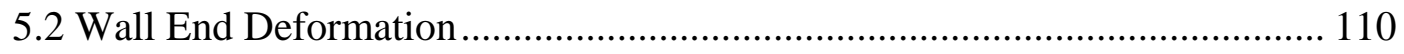

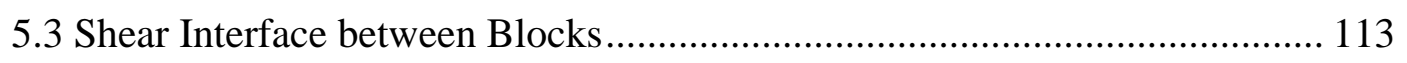

5.3.1 Sliding Between Blocks ....................................................................... 114

5.4 Shear Panel Rotation and Deformation.......................................................... 116

5.5 Shear Transfer along Sill and Top Plates........................................................ 119

5.5.1 Sill Plate Shear Transfer ...................................................................... 120

5.5.2 Top Plate Shear Transfer ........................................................................ 121 
5.5.3 Actuator to Top Plate Connection. 122

5.6 Failure of Screws and Nails Attaching Shear Panels...................................... 122

5.7 Global Relaxation of Shear Wall Specimens.................................................. 125

5.8 Development of Backbone Curves …………………..................................... 131

5.8.1 Prior Investigations into Backbone Curve Construction .......................... 132

5.8.2 Backbone Curve Generation for Straw Block Specimens ......................... 132

5.8.3 Backbone Comparison to Wood Framed Walls ........................................ 136

5.9 Hysteretic Dissipation of Energy …………………..................................... 139

Chapter 6 Development of Constitutive Modeling ................................................... 148

6.1 Hysteretic Backbone Analysis ......................................................................... 149

6.1.1 Linear Approximation of Backbone ........................................................ 149

6.1.2 Backbone Characteristics ...................................................................... 151

6.1.3 Comparison with Stud Wall Backbone Properties.................................... 152

6.1.4 Peak-Oriented Hysteretic Model........................................................... 154

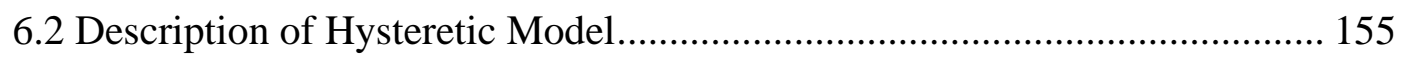

6.3 Discussion of Ductility Demand Limit States................................................... 157

6.3.1 ASCE/SEI 41 Limit States ................................................................. 158

6.4 Potential Shear Wall Design Strengths ........................................................ 161

6.4.1 Drift at Design Strength Approximation ................................................... 162

6.4.2 Force at 2.5\% Drift Approximation ........................................................ 163

6.4.3 Hysteretic Energy Dissipated at 2.5\% Drift Approximation.................... 165

6.4.4 Design Strength Approximation Results .................................................. 167

Chapter 7 Conclusions and Recommendations ........................................................ 169

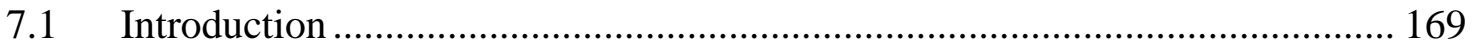

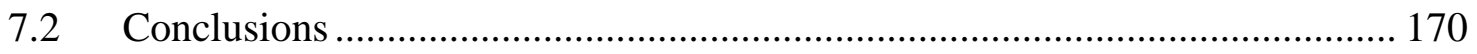

7.2.1 Materials Testing Conclusions................................................................ 170 
7.2.2 Structural Wall Construction Conclusions ........................................... 171

7.2.3 Structural Wall Testing Conclusions .................................................. 172

7.3 Recommendations for Future Work ......................................................... 174

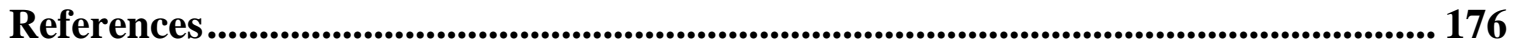




\section{List of Tables}

Table 1-1: Annual Rice Straw Production (US Dept. of Agriculture, 2008) ..............6

Table 2-1: Ash et al. (2003) Testing Results .......................................................16

Table 3-1: Summary of Compression Testing Program .......................................27

Table 3-2: Comparison to Prior Block Testing ..........................................................28

Table 3-3: Comparison of Half to Whole Blocks ................................................... 30

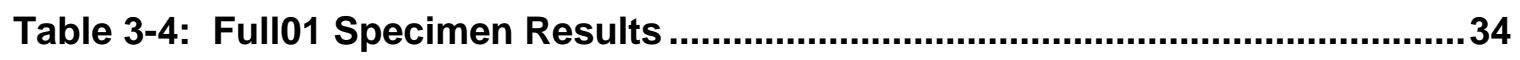

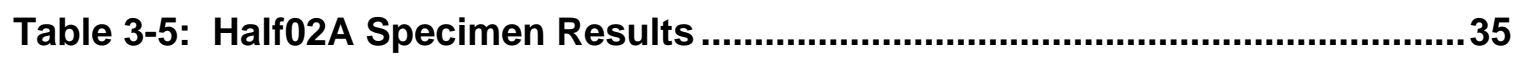

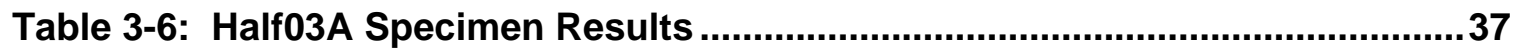

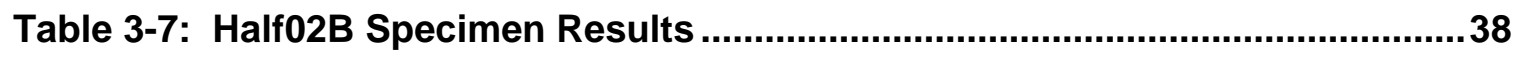

Table 3-8: Half03B Specimen Results .............................................................. 40

Table 3-9: Half04D Specimen Results ................................................................... 40

Table 3-10: Summary of Preliminary Material Testing Results.............................42

Table 3-11: Estimated Relaxation Losses........................................................44

Table 3-12: Used Block Testing Program ......................................................... 47

Table 3-13: Used Block Test Results ............................................................... 47

Table 3-14: Block Modification Effects on Strength and Stiffness..........................48

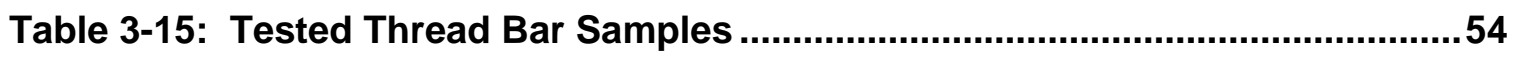

Table 3-16: ASTM F1554 Threaded Bar Grades......................................................54

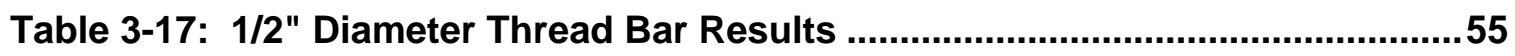

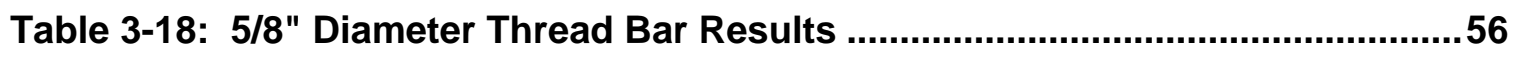

Table 3-19: Embodied Energy of Materials Used ...........................................60

Table 3-20: Bamboo Material Properties................................................................61

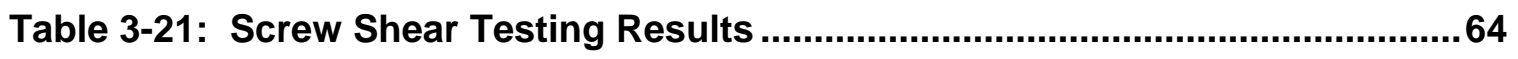

Table 3-22: Shear Interface Testing Results.....................................................70

Table 4-1: Shear Wall Testing Program................................................................. 72

Table 4-2: CUREE-Caltech Loading Protocol for a 97 inch Tall Wall ......................78

Table 4-3: Material Properties Used in FEM.......................................................... 82

Table 4-4: FEM Calculated Deflections at Top of Wall............................................ 86

Table 4-5: Shear Wall Testing Summary .............................................................100 
Table 5-1: Maximum Forces within Prestressing in Walls 1 and 2........................105

Table 5-2: Maximum Forces within Prestressing in Walls 3 and $4 \ldots \ldots \ldots \ldots \ldots \ldots . . . . . . . . . . . . .108$

Table 5-3: Wall 2 Panel Vertical Deformations ................................................... 118

Table 5-4: Sill Plate Slips for Various Details ................................................. 121

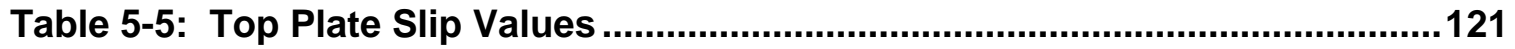

Table 5-6: Prior Shear Wall Testing Descriptions ...............................................137

Table 6-1: Positive Backbone Characteristics..........................................................151

Table 6-2: Percentage Difference in Backbone Characteristics .............................151

Table 6-3: Positive Backbone Ductility Parameters ................................................152

Table 6-4: Peak-Oriented Model Parameters ..........................................................155

Table 6-5: Calculated Ductility Demand Limit States per ASCE/SEI 41 ................160

Table 6-6: Design Shear based on Drift @ Code-Allowable Shear.........................163

Table 6-7: Design Shear based on Forces @ 2.5\% Drift..........................................165

Table 6-8: Design Shear based on Dissipated Energy @ 2.5\% Drift ......................166 


\section{List of Figures}

Figure 1-1: Straw Block Specimen ................................................................

Figure 1-2: Straw/Binding Agent Tumbler ..........................................................4

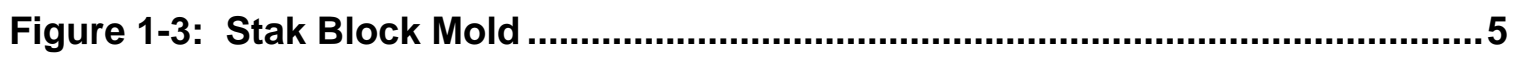

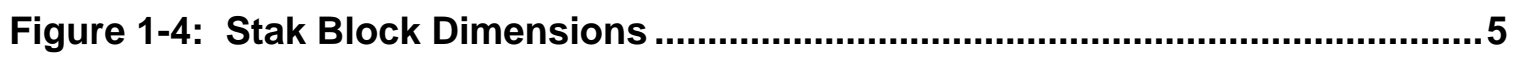

Figure 2-1: Bou-Ali (1993) Straw Bale Compressive Stress-Strain Relationships. 12

Figure 2-2: Northridge Earthquake Damage Example (FEMA News Photo, 1994).19

Figure 3-1: Sample Compression Testing Configuration .....................................23

Figure 3-2: Typical Failure at Higher Strains .................................................. 24

Figure 3-3: Elastic Modulus Approximation ....................................................... 26

Figure 3-4: Stress-Strain Behavioral Definitions..................................................26

Figure 3-5: Comparison of Half to Full Block Strengths .....................................29

Figure 3-6: Density versus Elastic Moduli .......................................................... 31

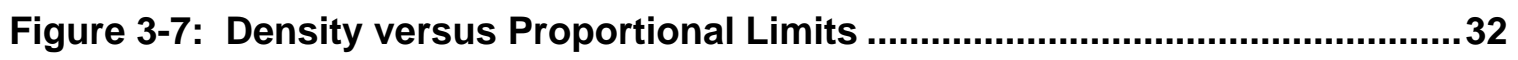

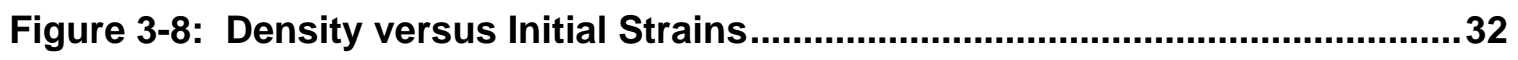

Figure 3-9: Full01 Specimen Results .......................................................... 34

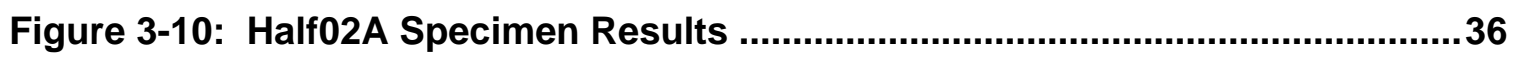

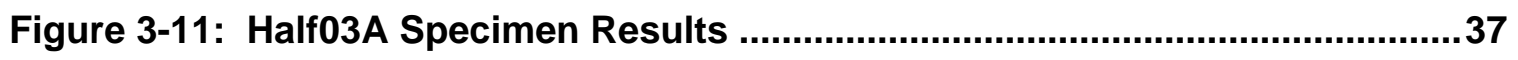

Figure 3-12: Half02B Proportional Limit Cycles ....................................................38

Figure 3-13: Half02B Specimen Results ........................................................ 39

Figure 3-14: Half04D Specimen Results ......................................................... 41

Figure 3-15: Summary of Preliminary Material Testing Results ........................... 42

Figure 3-16: Relaxation Testing Results ............................................................ 44

Figure 3-17: Effects of Atmospheric Changes on Block Relaxation .......................45

Figure 3-18: Comparison of Straw Blocks to Bales at High Strains.......................50

Figure 3-19: Comparison of Straw Blocks to Bales up to $12 \%$ Strain.....................51

Figure 3-20: Sample Prestressing Tensile Testing Configuration.........................53

Figure 3-21: 1/2" Diameter Thread Bar Results.................................................55

Figure 3-22: 5/8" Diameter Thread Bar Results....................................................56

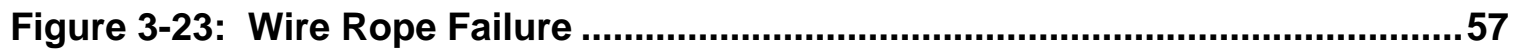


Figure 3-24: Comparison of Thread Bar to Wire Rope Prestressing.......................58

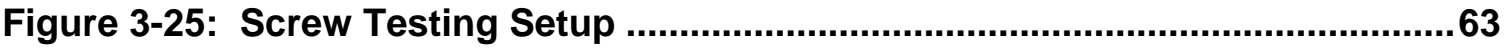

Figure 3-26: Nail Comparison Testing Setup........................................................64

Figure 3-27: Screw and Nail Shear Testing Results..............................................65

Figure 3-28: Bilinear Approximation of Screw Yield Points....................................66

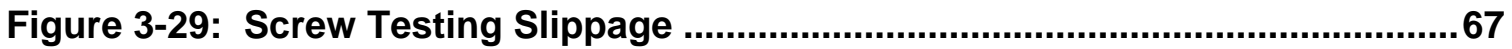

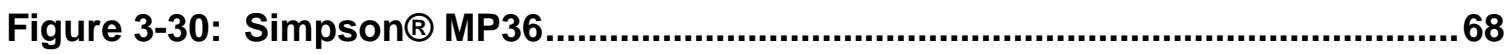

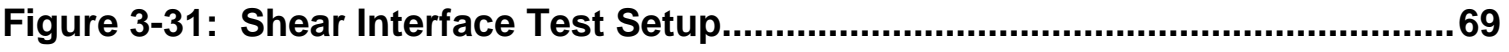

Figure 3-32: Shear Interface Testing Results ......................................................70

Figure 4-1: Laboratory Setup, Shear Walls 1 and 2 ...........................................73

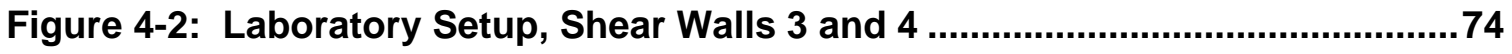

Figure 4-3: Moveable Foundation Detail ................................................................ 75

Figure 4-4: Shear Wall Measurement Instrumentation ..........................................76

Figure 4-5: Full CUREE-Caltech Loading Protocol ................................................ 79

Figure 4-6: FEM with Single Top Plate Loaded at Ends Only .............................83

Figure 4-7: FEM with Double Top Plate Loaded at Ends Only ................................. 84

Figure 4-8: FEM with Loading at Four Points ........................................................ 85

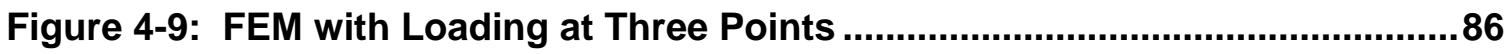

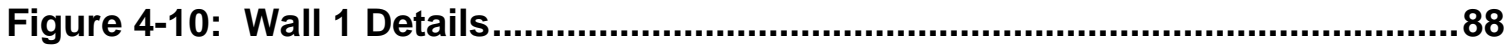

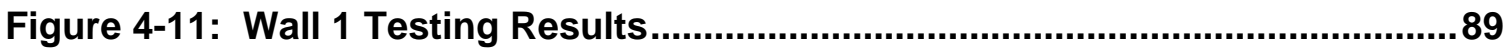

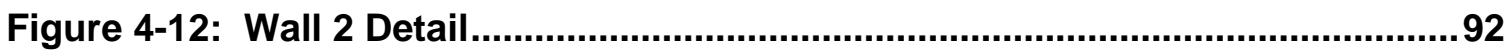

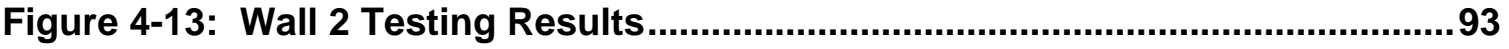

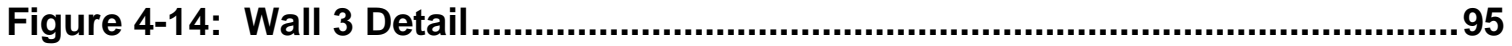

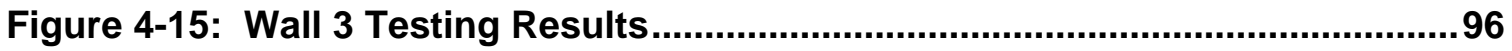

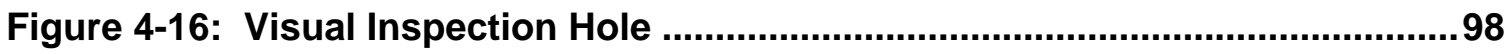

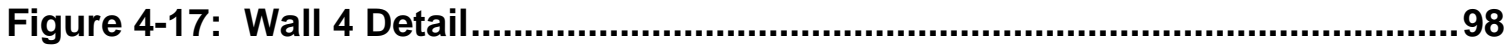

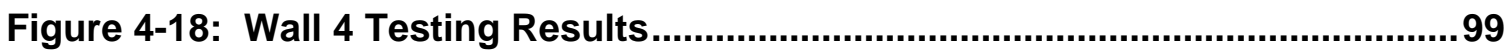

Figure 5-1: Free Body Diagram on Straw Wall .................................................. 102

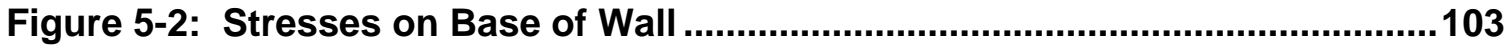

Figure 5-3: Summing Stresses at Base of Wall ........................................................104

Figure 5-4: Damage at Corners of Shear Panels ................................................... 107 
Figure 5-5: Thread Bar Elongation Due to Large Forces.....................................109

Figure 5-6: Wall 3 End Deformations ....................................................................... 111

Figure 5-7: Wall 4 End Deformations ............................................................... 111

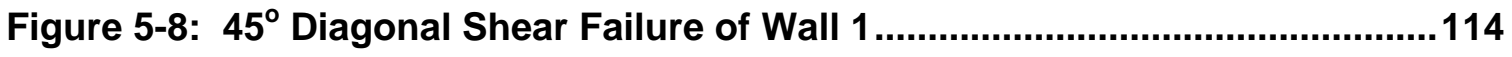

Figure 5-9: Typical Block Damage from Sliding ......................................................115

Figure 5-10: Gap Formed Opposite Panel Edges................................................116

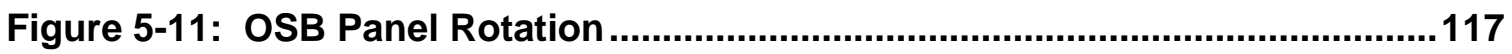

Figure 5-12: Wall 2 Panel Instrumentation...................................................... 117

Figure 5-13: Wall 2 Panel Diagonal Deformations....................................................118

Figure 5-14: Screw Head Protrusion Following Testing.........................................123

Figure 5-15: Straw Block Damage from Screw Movement .................................... 124

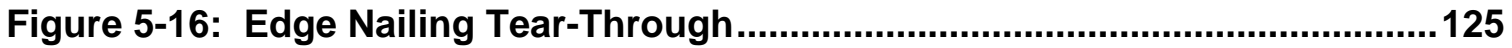

Figure 5-17: Wall 1 Relaxation Prior to Testing......................................................126

Figure 5-18: Wall 2 Relaxation Prior to Testing.................................................... 128

Figure 5-19: Wall 3 Relaxation Prior to Testing............................................. 129

Figure 5-20: Wall 4 Relaxation Prior to Testing......................................................130

Figure 5-21: Wall 1 Backbone Curve Construction............................................... 133

Figure 5-22: Wall 2 Backbone Curve Construction............................................... 133

Figure 5-23: Wall 3 Backbone Curve Construction................................................. 134

Figure 5-24: Wall 4 Backbone Curve Construction................................................134

Figure 5-25: All Four Walls' Positive Backbones.................................................... 135

Figure 5-26: All Four Walls' Negative Backbones..................................................136

Figure 5-27: Prior Shear Wall Testing Backbone Comparison ............................. 137

Figure 5-28: Example Hysteretic Energy Calculation .......................................... 140

Figure 5-29: Dissipated Hysteretic Energy for Wall 1 ..........................................140

Figure 5-30: Dissipated Hysteretic Energy for Wall 2 ...........................................141

Figure 5-31: Dissipated Hysteretic Energy for Wall 3 ........................................141

Figure 5-32: Dissipated Hysteretic Energy for Wall 4 .......................................142

Figure 5-33: Dissipated Hysteretic Energy for All Wall Specimens ......................143

Figure 5-34: Normalized Hysteretic Energy for All Wall Specimens .......................144

Figure 5-35: NHE for Wood Shear Walls from Dodge (2008).................................145 
Figure 5-36: NHE for Wood Shear Walls from Heredia (2010).............................145

Figure 5-37: NHE for Wood Shear Walls from Ibarra et al. (2005)........................146

Figure 6-1: Linear Representation of Backbone Curve .........................................149

Figure 6-2: Example Backbone Curve for Typical Hysteretic Models...................150

Figure 6-3: Basic Rules for Peak-Oriented Hysteretic Models................................154

Figure 6-4: Types of Nonlinear Wall Responses ..................................................159

Figure 6-5: Capacity Curve with ASCE/SEI 41 Performance Limit States ............160

Figure 6-6: Backbone Comparison @ Code-Allowable Shear ...............................163

Figure 6-7: Backbone Comparison @ 2.5\% Drift..........................................................164 


\section{Chapter 1}

\section{Introduction}

\subsection{Background}

Anthropologists believe that straws and grasses have been used in the form of adobe bricks or cob to build structures since pre-history, about 40,000 years (Chiras, 2000). The use of modular straw bale as a viable building material began in latenineteenth Nebraska where an emergency one-room schoolhouse was constructed (Wilson, 1995). This method provided an energy efficient, locally available, easily built, and practically free structure. These ideals are at the crux of the modern "green" construction movement. The United States Green Building Council (2005) has developed a rating system and standards program called the Leadership in Energy and Environmental Design (LEED). There is potential for a construction project using Stak Blocks to achieve up to thirteen points in this system (with 26 total needed for LEED certification, 33 for LEED Silver, and so on...) based on ideas such as energy efficiency, local sourcing, and rapidly renewable building products. 


\subsubsection{Historical Usage of Rice Straw}

Straw is appealing as a building material because it is a byproduct of the harvesting of grains such as wheat, rice, oats, barley and rye. In California, the usage or disposal of straw is changing. It used to be that rice straw was either burnt in the field or baled for later controlled burning. As recently as 1990, nearly 1.1 million tons of rice straw and an additional 124,000 tons of wheat and barley straw were burned in the state of California. This burning released 62,000 tons of Carbon Monoxide (CO) into the atmosphere. For comparison purposes, California power plants expelled 26,000 tons of CO over the same year (Jenkins, 1991).

Realizing something had to be done; the state of California passed the California Rice Straw Burning Reduction Act of 1991 which “mandates that rice straw burning in the Sacramento Valley be phased down starting in 1992 and, beginning in September 2001, allowed only under specified conditions for disease control.” (State of California Health and Safety Code, 1991). Now it is common to disk and plow straw under the soil for decomposition, or bale it for sale for a multitude of uses from animal bedding to straw bale construction. The "soil incorporation" process is estimated to cost the farmer approximately $\$ 40$ per acre (California Rice Commission, 2009) and still is not as effective as burning in limiting disease and weeds. This change in attitude and farming practice amounts to an abundance of a rapidly renewable potential building material available in California.

\subsubsection{Stak Block Product Manufacturing}

The art of structural design and the materials utilized are in constant flux and evolution. It is of this evolution that the Stak Block, an interlocking compressed straw 
block, came to be developed as a refinement of the straw bale. The Stak Block is a product patented by Oryzatech, Inc. (U.S. Patent 6,951,080 B2) using the culm (the stem or stalk) of a grass, in this case the cereal grain rice, to create a 12”x24”x12” structural block (Figure 1-1).

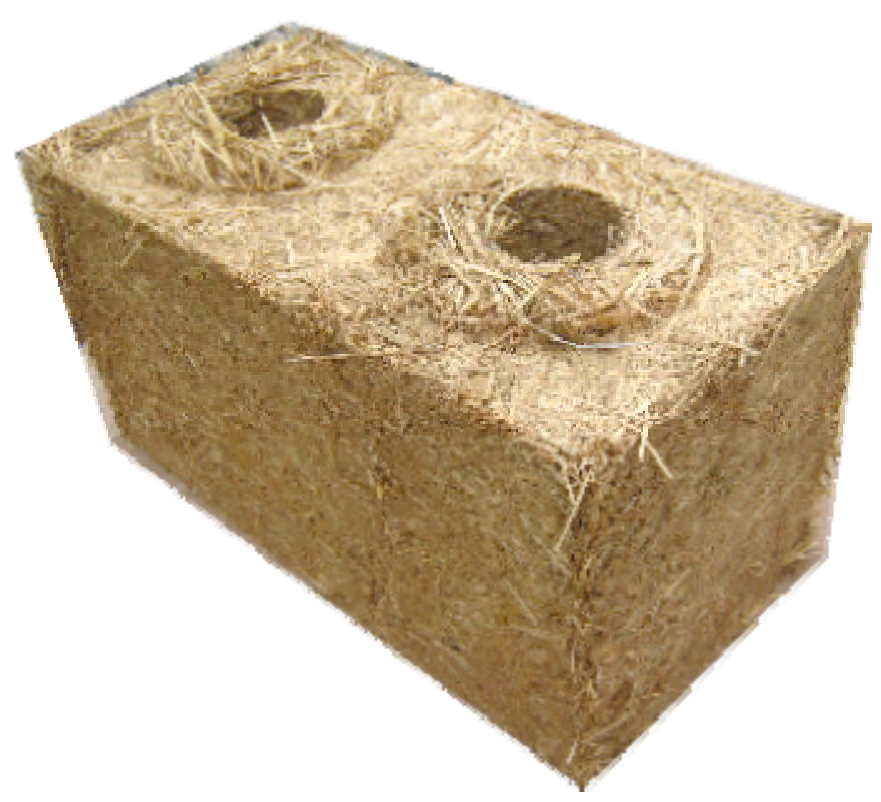

Figure 1-1: Straw Block Specimen

Oryzatech’s manufacturing process for each block starts with pulling loose straw from a rice bale and mixing in a large tumbler (shown in Figure 1-2) with a small amount of Methyl Diisocyanate Polyurethane (MDI) acting as a binding agent and moisture inhibitor (the same glue used to make oriented strand board or engineered lumber Ijoists). Rice straw is used in lieu of other grains because of its high silica content which provides superior strength and durability. The amount of MDI used in the process is so minute that the loose straw does not appear to be moist and the only way to verify even coating is with a chemical spray that turns red when combined with the MDI. 


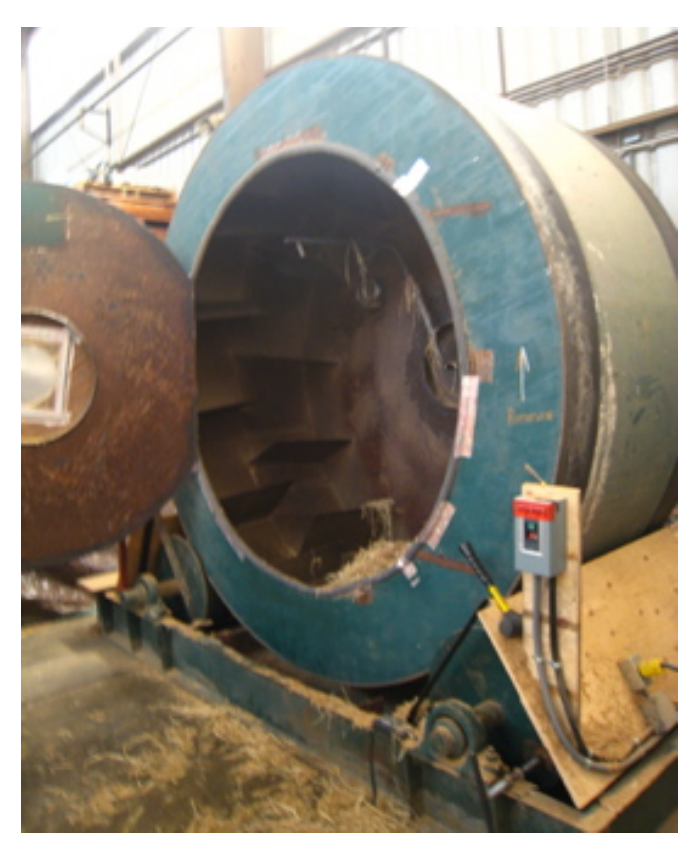

Figure 1-2: Straw/Binding Agent Tumbler

The next step in the procedure is placing approximately 30 pounds of straw into a mold attached to a hydraulic press. The machinery then compresses the straw mixture to a desired pressure and then the mold is assembled and placed into an oven for about an hour. The temperature in the oven is just high enough to allow for the binding agent to set. The cylindrical voids were incorporated to reduce the volume and allow for the blocks to heat up and cool down evenly and quickly. The steel mold (shown in Figure 13) is then removed and the finished product is a Stak Block, detailed in Figure 1-4. The current manufacturing process allows for four blocks to be produced at one time at a cost of approximately \$7-15 per block. If a more automated system were used, it is estimated the price per block would drop to $\$ 7.50-\$ 8.50$. 


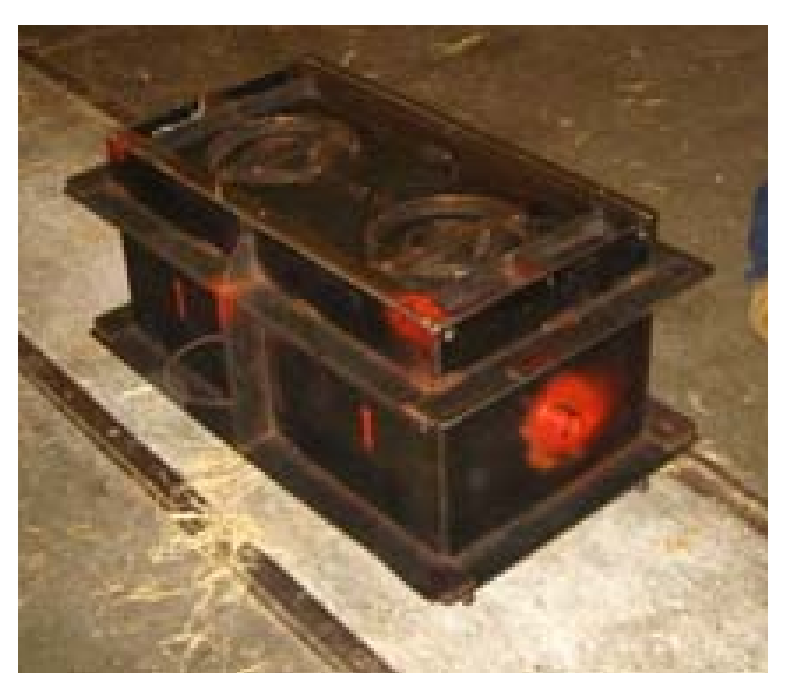

Figure 1-3: Stak Block Mold
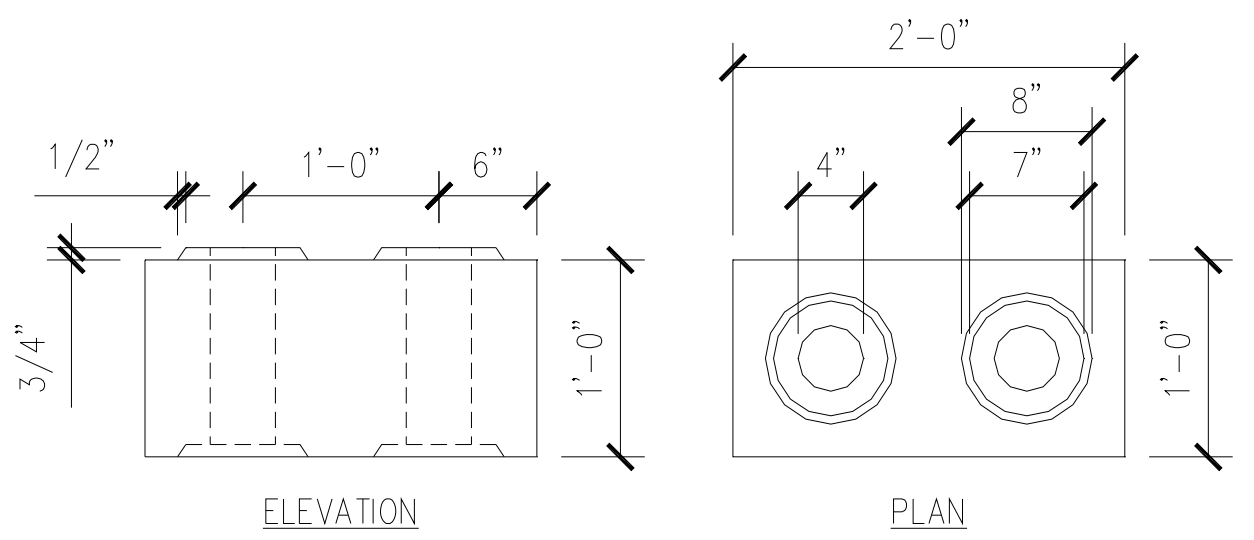

Figure 1-4: Stak Block Dimensions

\subsubsection{Potential for Stak Block Production}

This manufacturing process allows for the reappropriation of a current agricultural byproduct as a primary structural element in new construction projects. For example, in 2008, enough straw was produced in California alone (US Dept. of Agriculture, 2008) to hypothetically manufacture over 120 million straw blocks for construction (Table 1-1) which would be enough to build the exterior walls of 200,000 1,000 sq ft single story homes. 
Table 1-1: Annual Rice Straw Production (US Dept. of Agriculture, 2008)

\begin{tabular}{ccc} 
& Nationwide & California \\
\hline \hline $\begin{array}{c}\text { Area Harvested } \\
\text { (million acres) } \\
\text { Grain Yield } \\
\text { (tons/acre) }\end{array}$ & 2.976 & 0.517 \\
$\quad 3.423$ & -- \\
$\begin{array}{c}\text { Straw Yield } \\
\text { (tons/acre) }\end{array}$ & 3.347 & -- \\
$\begin{array}{c}\text { Total Straw } \\
\text { (million tons) }\end{array}$ & 9.96 & 1.73
\end{tabular}

While it is not realistic to divert all produced straw to block manufacturing, the fact that so much material is readily available for use in construction is very encouraging.

The question remains though: why not simply use baled straw in construction instead of producing the blocks investigated in this report? The use of straw bales in construction has become more and more commonplace over the past two decades; recently local building codes have even begun adopting provisions for construction with straw bales.

On a basic level, there are two categories of straw bale construction: load bearing, where the bales are used to carry vertical loads in compression; and post and beam, where the bales are used as infill between timber framing members that carry the structural loads. The latter type is more common in construction due to uncertainty surrounding the load bearing and lateral force resisting ability of a straw bales and their tendency to creep over time and cause cracking of the architectural finishes.

\subsection{Objectives and Scope}

The objective of this work is to develop and test the detailing for a combined load bearing and shear force resisting structural system using Stak Blocks. The use of this straw-derived building material would be a viable from a structural and constructability 
standpoint in regions of the United States where both straw is prevalent and where moderate to extreme seismicity may be expected.

Various mechanical properties of the Stak Blocks are to be investigated to the extent of developing details of the structural wall systems. Materials associated with the prestressing of the wall system as well as interfaces between conventional building materials and the new straw composite surface are to be tested and quantified. The results from these material tests are to provide insight in the development and subsequent iterative process of detailing and constructability advancement.

Four 8 foot by 8 foot walls using various iterations and levels of detailing will be constructed and tested. The behavior and performance of each wall will be monitored to assess the viability of prestressing the system as well as various construction decisions and methods. Testing of the four walls should provide insight into the best methods and even possible pitfalls in future construction using Stak Blocks.

The data collected during testing will allow for the comparison to similarly detailed and tested timber framed shear walls. These test results are to be used to predict the performance of theoretical buildings (residential structures) under various earthquakes, both recorded ground motions and probabilistic design earthquakes. The findings from this analysis will be used to compare the ductility performance of structures constructed with Stak Blocks to conventional timber stud framed walls to evaluate the feasibility of using straw blocks as a structural system.

\subsection{Organization and Contents}

The research described herein investigates how the straw blocks can be used as both the vertical and lateral force resisting systems. First, in Chapter 2, findings from 
previous studies on the use of straw bale as a building material are compared to one another. These tests include the strength and stiffness of unplastered and plastered bales as well as the in-plane cyclic behavior of constructed straw bale wall systems. Also, literature will be presented on the development of a loading protocol in which the behavior of a design-level earthquake is most accurately simulated.

Next, in Chapter 3, a materials investigation is performed to determine material properties of the blocks ranging from basic stress-strain behavior to measurements of yield strength and elastic stiffness. Additionally, the relationships between strength and density as well as more in-depth characteristics such as cyclic loading behavior and longterm relaxation will be reported. The materials investigation will also delve into various prestressing material options and their associated strengths as well as fastening methods to attach sheathing and the shear interface strength between interlocking Stak Blocks.

Chapter 4 will describe the testing setup, instrumentation, and pseudo-static CUREE-Caltech loading protocol used in cyclic shear wall testing. Additionally, a discussion of the decision to prestress the wall system will be presented as well as complete construction details for each of the four wall specimens. Finally, a basic testing summary for each wall tested will be included.

Chapter 5 will present the results from the full-scale shear wall testing program. Results from the various detailing design iterations will be quantified and compared with data collected during testing. In addition to this, basic visual observations and failure modes of different components will be presented for each wall system tested. The results from full-scale wall relaxation during the time prior to testing will be discussed and compared to the findings from the individual block testing. Next, the dissipation of 
system hysteretic energy will be calculated and included to allow for comparison between all four wall specimens. Finally, from the force-displacement hysteresis data, phenomenological backbone curves were generated for each wall test. These backbone curves will then be compared to the data from similarly detailed and tested wood framed shear walls to assess the available ductility supply.

Next, Chapter 6 will illustrate the development of a model that could be used to perform a nonlinear analysis. The simplified model for the straw block walls will allow for comparison with similar wood framed walls. Various limit states that can be used to quantify ductility and seismic performance will be compared and contrasted. This analysis will allow for the feasibility of using Stak Blocks as a load bearing and lateral force resisting system to be studied. Additionally, preliminary design shear values for the Stak Block walls will be determined using various methodologies. Chapter 7 will conclude by summarizing the findings of this report and will provide recommendations for future research using this new straw block building material. 


\section{Chapter $2 \quad$ Literature Review}

One of the consequences of using a newly patented, still-in-development building material such as Stak Block is the complete lack of published research on the topic. Small scale research had been performed in the same laboratory used in this study to determine the most effective mix proportions for manufacturing purposes, but no prior investigation into the structural behavior of these blocks was available. As mentioned in Chapter 1, the Stak Block was developed because of constructability issues surrounding conventional straw bales. Because of their irregular shapes, lack of quality control, and relatively poor performance in testing, building departments and contractors treat the straw bales with trepidation.

Given that the Stak Block is seen as an evolution or improvement of straw bales, the small amount of research that has been conducted on bales and will be presented for comparison purposes. Additionally, the aim of Stak Blocks is to be used in lieu of standard timber framed construction in smaller scale projects and because of this, the 
following chapter provides some information regarding the testing, performance, and analysis of stud framed shear walls.

Although straw has been used as a building material in the form of adobe for thousands of years and as modular bales for over one hundred, research and testing has only followed the resurgence of straw bales in the past twenty years. While much of this research has focused on full-scale load-bearing plastered straw bale walls, some of the earlier research investigated the strength of straw bales on a material level.

In the interest in moving towards code acceptance of a proprietary material such as the straw blocks being investigated in this study, a comparison to conventional timber framed construction was performed. Several research publications from projects involving timber structures and their dynamic behavior provide a basis from which testing procedures were developed for this study.

\subsection{Straw Bale Construction}

\subsubsection{Basic Testing of Straw Bales and Straw Bale Wall Systems}

Bou-Ali (1993) investigated wheat straw bales and straw-bale wall systems loaded axially, in-plane, and out-of-plane. Single unplastered bales were loaded flat (perpendicular to their largest face) and on edge (parallel to their largest face). For flat use, an ultimate compressive strength of 84 psi was reported at over $50 \%$ strain as well as “elastic” moduli ranging from 78 to 211 psi and a Poisson’s Ratio of 0.30. Figure 2-1 below shows the stress-strain results for the four flat loaded bales reported by Bou-Ali. 


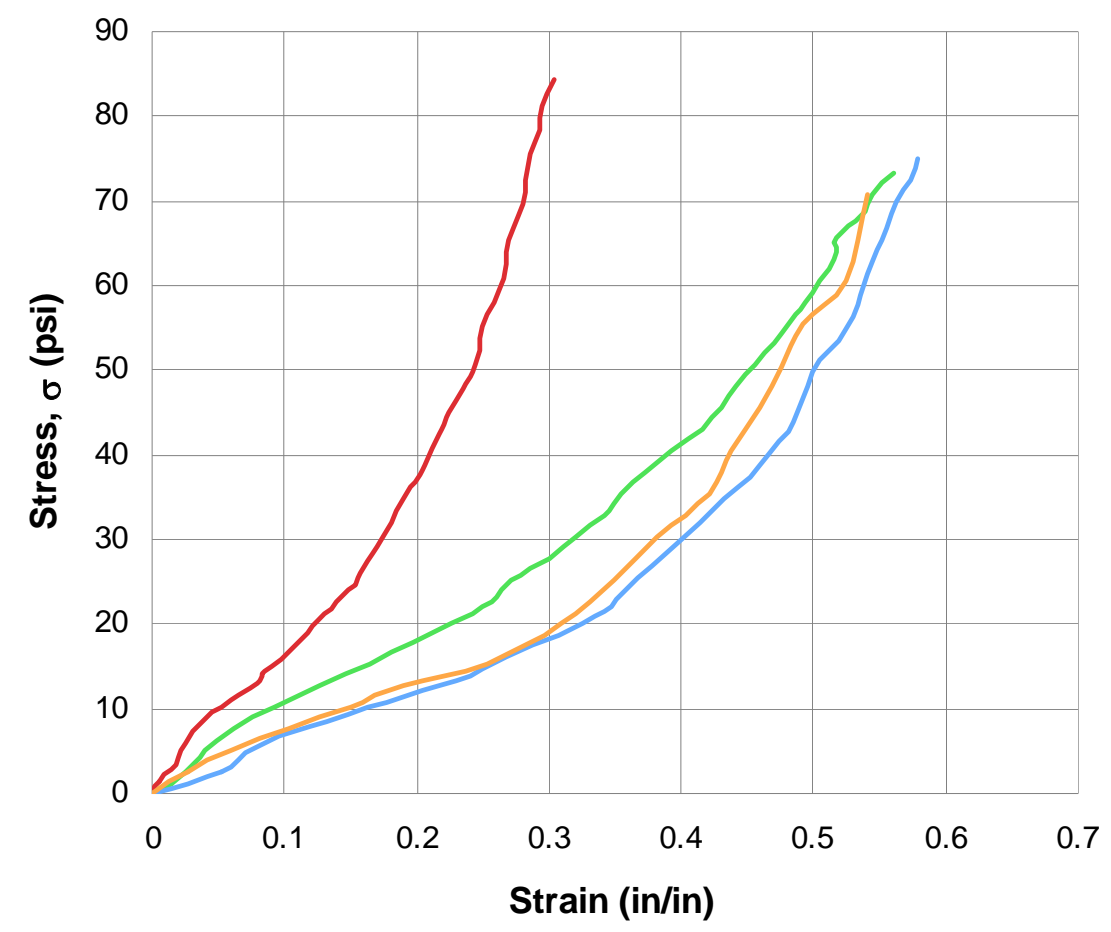

Figure 2-1: Bou-Ali (1993) Straw Bale Compressive Stress-Strain Relationships

The plot above shows the clear strain-hardening behavior exhibited by the straw bales. One issue with the results Bou-Ali reported is the elastic moduli values ranging from 78 to 211 psi appear to have been measured well beyond the "elastic" region. Also the strengths reported were the ultimate strengths were string breaking occurred. These strengths were obtained at strains around 50\% which is unreasonable in any type of construction. A more reasonable allowable stress could have been obtained around 2, 5, or even $10 \%$.

The bales loaded on edge failed suddenly at a load of 21 psi due to the rupturing of the binding strings but had a similar range of elastic moduli as those loaded flat. The author also investigated the relation between bale density and compressive strength. It 
was reported that with straw bales, there is a positive correlation between density and stress at failure.

The second phase of Bou-Ali's (1993) testing program was the construction of nine full scale wall panels measuring 12 feet long by 8 feet high. Three walls were loaded axially to a load of 1317 plf (due to testing machine limitations) and had vertical deflections ranging from 6.9” to 7.8”. All wall specimens experienced a buckling failure mode and concluded that straw bale walls had a load bearing strength comparable to that of masonry in pounds per linear foot.

The next six wall tests were loaded laterally; three in-plane and three out-ofplane. The walls were all unplastered and were reinforced with \#4 rebar pins spaced at 24”. The out-of-plane walls were loaded with incremental distributed pressures ranging from 5 psi (which showed no deflection) to 23 psi (which showed up to one inch of deflection). Walls were tested in-plane in a similar manner of incremental load increases. The results of the three wall specimens varied a great deal with one wall loaded to 2135 pounds and deflecting 6.0 inches at the top plate, while another, constructed to the same specifications, only deflected 2.3 inches under the same load. Bou-Ali concluded that the walls could safely withstand a lateral load of 26 psf, which was reported to be equivalent to a $100 \mathrm{mph}$ wind gust. The author also commented on the variability seen in baling techniques and sourcing and thus warned against extrapolating the reported findings to other straw bales.

Stephens and Budinger (2000) performed compressive testing on “supercompressed” bales that are made for overseas export. These bales are about twice their normal density due to specialized heavy hydraulic machinery used in the 
manufacturing process. These bales were loaded on edge and exhibited classic linear behavior up to a proportional limit of 17 psi with 0.5 inches of deflection (over 24” height or 2\% strain). An elastic modulus of 992 psi was reported, which shows the effects of density on strength and stiffness.

Blum (2002) investigated wall assemblies made of both wheat and oat bales. The wheat bale walls buckled under an average load of 319 plf and the oat performed much better buckling under 617 plf. The author also reported that the wheat bale walls exhibited drastic stiffness creep, losing half of their resistance under a load left in place for three days.

\subsubsection{Investigations into Plastered Straw Bale Construction}

Due to the fact that the standard of practice in construction is to plaster straw bale walls, the majority of testing has focused on this method. Zhang (2000) reported an initial set phase (3 to $4 \%$ of the wall height) during testing in which the "fluff" between bales has to be compressed before applying any plaster. Platts and Chapman (1996) addressed this "fluff" by prestressing the wall specimens to 700 plf, which reduced the height of the wall by 3”, or 3\%, before applying stucco. These wall specimens were loaded to an initial load of 2944 plf (calculated service load for two stories) and then to 4500 plf (the limit of the testing apparatus). The testing showed that the tensioning of the mesh greatly increased the shear resistance as well as compression properties of the bales. Prestressing also allowed for final leveling of the walls and provided substantial construction stability and safety.

Vardy (2009) performed a study collecting the majority of available data on straw bale testing, both plastered and unplastered, as well as their own testing program. The 
author determined that using accepted construction practices, typical residential loads will not yield plastered straw bales. Further investigations into mix design sought to determine effects on the wall system from having stucco of various strengths. The testing results from this investigation for plastered straw bales loaded flat yielded ultimate loads ranging from 1500 to upwards of 6000 plf in compression. The author also developed an equation to calculate the design strength of a plastered straw bale wall based on assumptions on the strength of the plaster, a specific strain at failure, and a predictable elastic modulus for the straw bale. This equation yielded a design force of approximately 1400 plf.

Ruppert and Grandsaert (1999) tested three types of barley bale wall assemblies by varying the bale thickness and type of reinforcing mesh on which the plaster was applied. Counter-intuitively, the 24” bale walls failed at an average of 3415 plf, while the 18” bale walls with the exact same detailing failed at 6156 plf. This dramatic difference was not understood and has yet to be explained, but shows the inherent variability in straw bale composition. Faine and Zhang (2002) constructed one and two-story walls of wheat bales detailed in a manner that the much stiffer plaster skins were free to slide at the supports. The one-story wall yielded at 1233 plf and failed under an ultimate load of 2467 plf while the two-story wall yielded at 1919 plf and failed at 3221 plf.

\subsubsection{In-Plane Cyclic Testing of Plastered Straw Bale Wall Assemblies}

Ash et al. (2003) performed in-plane cylclic tests on a series of six plastered straw bale wall assemblies. Of the six walls tested, three were constructed using earthen plaster with light, medium, and heavy reinforcing detailing and the other three had cement 
stucco skins with the same varied details. The lighter detailing was to be used as a baseline comparison to the medium (designed to yield at the base) and heavy (confined first coursed to shift the flexural yielding higher on the wall). Each wall was eight feet by eight feet and was precompressed overnight by attaching weights to the header beam to simulate dead load to at least 200 plf before applying the nominal $11 / 2$ ” thick stucco skin.

The bales used in the walls varied in density from 6.4 to 8.0 pcf (in construction, the standard recommended minimum of $7.0 \mathrm{pcf}$ ). The lightly detailed earthen plaster wall was tested using a monotonic loading protocol, while the other five walls were tested using a cyclic testing protocol with peak displacements at prescribed drift increments followed by multiple trailing cycles. The results of the testing program are found below (Table 2-1).

Table 2-1: Ash et al. (2003) Testing Results

\begin{tabular}{cccc} 
Wall Detailing & $\begin{array}{c}\text { Peak Load } \\
\text { (kips) }\end{array}$ & $\begin{array}{c}\text { Drift @ } \\
\text { Peak Load } \\
\text { (\%) }\end{array}$ & $\begin{array}{c}\text { Load @ } \\
2.5 \% \text { Drift } \\
\text { (kips) }\end{array}$ \\
\hline \hline Light, earth plaster & 3.2 & 4 & 2.85 \\
Medium, earth plaster & 4.7 & 1 & 3.7 \\
Heavy, earth plaster & 6.1 & 1.5 & 5.4 \\
Light, cement stucco & 6.4 & 1.5 & 6 \\
Medium, cement stucco & 19 & 2 & 17.6 \\
Heavy, cement stucco & 17.9 & 2 & 10
\end{tabular}

The results above show that the cement stucco performed better than the earth plaster. Also, it was reported that ductile tension failures of the reinforcing mesh were obtained only with the cement stucco skinned walls. The earth plaster skinned walls predominantly saw slippage of the top and base plates relative to the bales and subsequent crushing of the plaster. The heavier detailed walls saw failures of the mesh 
near the attachment to the sill plate as well as cross grain bending failure of the plate itself. Overall, all walls displayed stable response at high drift levels with no indications of collapse imminent.

\subsubsection{Fire Testing}

Beyond structural investigations, various tests have been performed on nonstructural material properties of straw bale. Inertek (2007) performed fire tests per ASTM E 119-05a on a $10 \mathrm{ft}$ by $10 \mathrm{ft}$ non-loadbearing wall constructed with $7.5 \mathrm{pcf}$ wheat straw bales clad in 1” of cement stucco. The wall successfully met the conditions of acceptance stipulated by ASTM E119-05a to achieve a fire endurance rating of 2 hours. Although concerns about flammability will surface when straw construction is discussed, the tests have proven that the exterior layer to char, and because of the density of the straw bales, no oxygen can reach the interior core of the bales and allow thorough burning.

\subsubsection{Thermal Properties}

One of straw's biggest selling points is its superior thermal insulation capacity. McCabe (1994) tested unplastered rice and wheat bales with a hot plate and thermal couples to find R-values ranging from 2.38 to 3.15 per inch of thickness (R-54 to R-71 for three string bales, R-39 to R-52 for two string). Watts, et al (1995) performed in situ tests on plastered straw bale walls and calculated an average R-value of 1.48 per inch (R28.4 for 18.4” thick wall and R-33 for 23” thick). Since the tests were performed in situ, density of the bales could not be measured. Christian (1998) conducted more tests focusing on closely replicating the actual conditions of walls in home constructions. 
These wheat bales had higher moisture contents than typically seen in laboratory testing, but an R-value of 1.45 per inch was determined (R-33 for 23” bales).

From these various thermal tests, the California Energy Commission officially regards an R-value of 30 for plastered straw bale walls. Although higher values have been reported, Stone (2003) has stated that above R-30, the windows, floor and doors overshadow the straw’s superior insulation. For comparison purposes, conventional wood framed construction with fiberglass insulation is assumed to have an R-value ranging from 12 to 19 , so straw bale is a much more energy efficient option.

\subsection{Development of Testing Protocol and Model for Analyzing Timber Structures}

As part of the CUREE-Caltech Woodframe Research Project, Krawinkler et al. (2001) developed a pseudo-static loading protocol to analyze the seismic response of timber structural systems. The loading protocol would simulate the behavior of a structure subject to design level earthquakes, or those with a probability of exceedence of $10 \%$ in 50 years.

The CUREE-Caltech Woodframe Research Project was initiated following the January 1994 Northridge earthquake in the Los Angeles area of California. According to the Federal Emergency Management Agency, approximately 114,000 residential and commercial structures were damaged and 72 deaths were attributed to the earthquake. Damage costs were estimated at \$25 billion (National Science Foundation, 2009). Figure 2-2 shows an example of the damage seen in residential structures. 


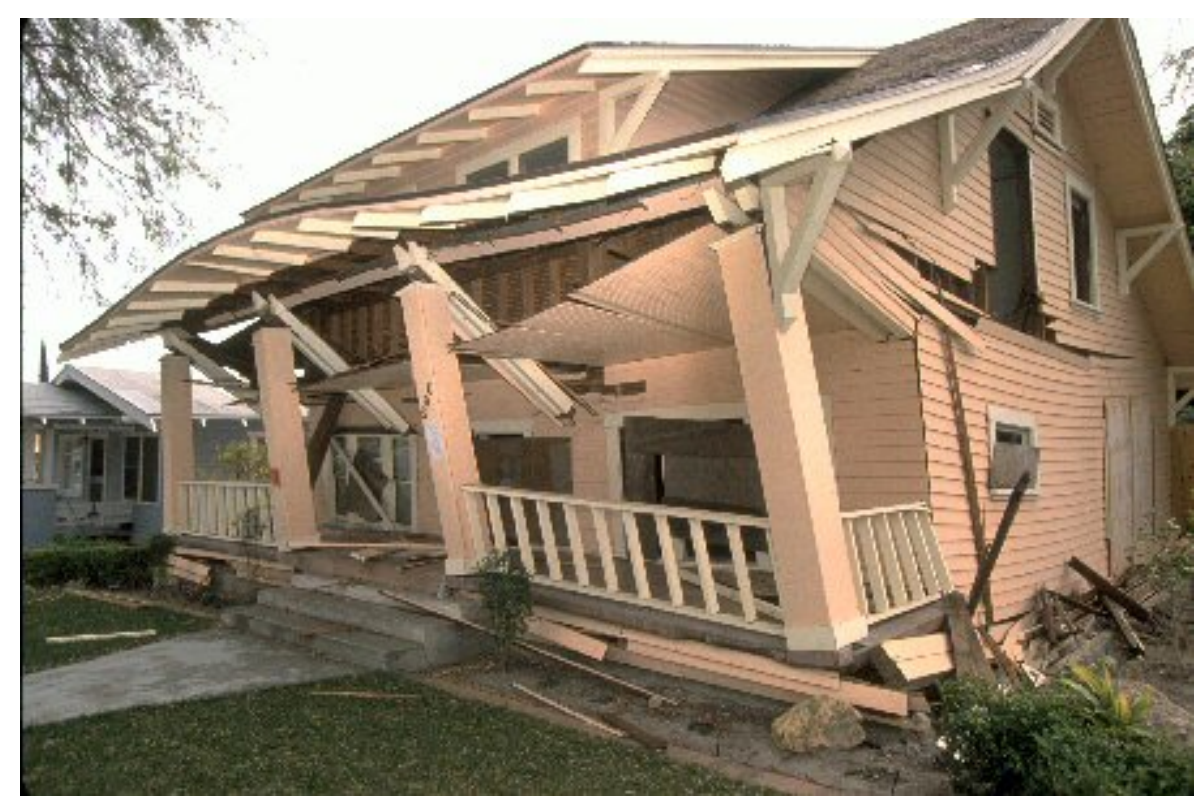

Figure 2-2: Northridge Earthquake Damage Example (FEMA News Photo, 1994)

The CUREE-Caltech Woodframe Research Project aimed to develop

methodology for both pseudo-static and dynamic testing of timber systems. The research sought to simulate realistic loading conditions in the laboratory in the testing of timber structures and their associated structural components. The methodologies developed would allow for practical laboratory investigations and proper design of structures to mitigate loss in future seismic events.

The loading history developed would consist of three categories of deformation cycles: initiation, primary, and trailing. The initiation cycles are executed at the beginning of the loading history and serve to check loading equipment, measurement devices, and to remove residual stress accumulated during installation into the testing apparatus. Primary cycles (larger than all preceding cycles) would provide the significant movement and multiple secondary cycles (equal to $75 \%$ of the amplitude of the preceding primary cycle) would simulate intermediate movement. 
The authors noted that their research findings showed the developed pseudo-static loading protocol was able to closely match the dynamic response of a structural system during a design-level earthquake. Gatto and Uang (2003) performed tests on wood frame shear walls using various loading protocols and also found the CUREE protocol to be most consistent with seismic behavior.

Ibarra et al. (2005) calibrated and developed hysteretic models that incorporate strength and stiffness deterioration. These properties or features of a structure are critical for demand predictions as a system approaches collapse during an earthquake event. Simplified linear hysteretic models were developed based on experimental testing of steel, plywood, and reinforced concrete specimens. The CUREE-Caltech pseudo-static loading protocol, as well as the previously developed Sequential Phased Displacement protocol, was used in testing.

Backbone curves were developed that were defined by the elastic (initial) stiffness, the yield strength, and the strain-hardening stiffness. If cyclic deterioration was evidenced during testing, a softening branch begins at the peak deformation and usually has a negative value. Various models were investigated looking at materials testing showing no cyclic deterioration and those that exhibited deterioration in the form of hysteretic energy dissipation. This cyclic deterioration was defined by the parameter $\beta$. In each tested system, observed behavior closely matched the developed hysteretic model. 


\section{Chapter 3 Materials Investigation}

In order to investigate the feasibility of using the Stak Blocks in structural applications, basic material properties first had to be determined. A series of tests was performed to determine constitutive properties such as their available stresses beyond serviceability, stress-strain relationship, and behavior under cyclic loading as well as the inherent variability in a manufactured product of natural materials.

For comparison purposes, the reported strengths of conventional straw bales served as a baseline in looking at the results of the compressive testing. Although not much research has been performed on straw bales in the areas of long-term creep or relaxation, this investigation is vital in dealing with a relatively "soft" material in conjunction with more rigid timber structures.

Beyond the materials investigation included in this report, constructability issues and details must be figured out for this new building material and method. These construction details and the iterations involved in this project will be discussed later, but various materials used in this structural system also had to be tested to obtain material 
properties. These material properties allowed for various behaviors in this structural system to be quantified and provided insight into the response of the shear wall.

Testing of all of the following properties, from compressive to screw shear strength was performed on a 120 kip capacity MTS test frame in the Cal Poly Advanced Materials Laboratory. Since no ASTM testing method has been established for straw bale or straw derived materials, a simple compressive testing program was established to investigate the desired properties.

\subsection{Measurement of Straw Block Constitutive Properties}

For compressive testing, a 3/4 inch plywood sheet measuring 13 inches x 25 inches was placed underneath the specimen to ensure a flat and even surface to provide uniform load distribution. Since the top of each block has two studs that provide interlocking shear strength when stacked, another 1/2 inch plywood sheet with two holes cut to allow for the surface adjacent to the raised studs to be loaded. One more plywood sheet was placed on top of the plate with holes to provide an even loading surface. A custom-made steel frame consisting of 3/8 inch thick plate and three tube steel members was used to provide rigidity to evenly transfer load from the cylindrical test head to the entire surface of the block. 


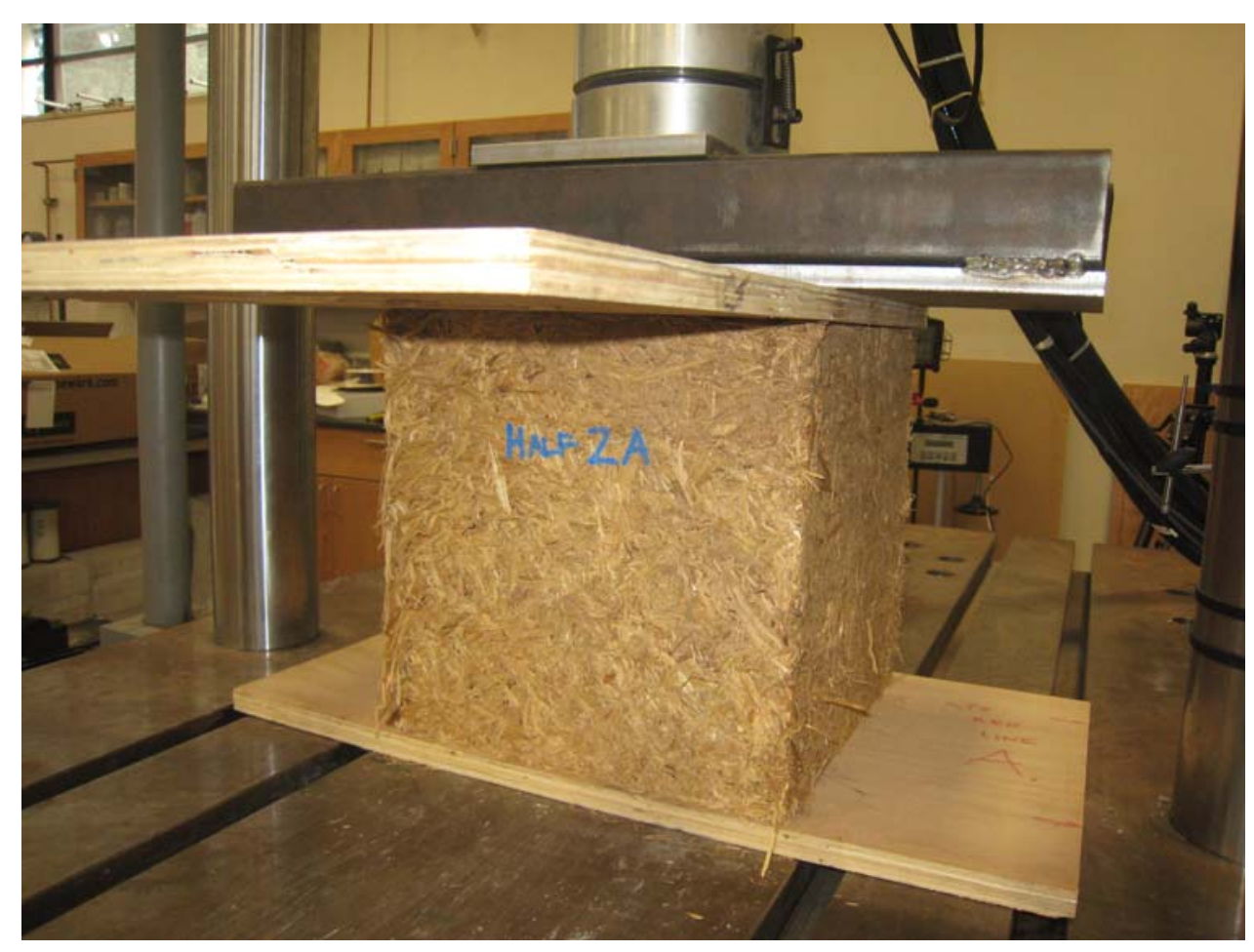

Figure 3-1: Sample Compression Testing Configuration

The compressive tests described below all were performed using a displacement controlled test program that would load at 0.12 inches per minute up to a prescribed displacement or strain and then unload to zero displacement. Cyclic tests use this same load rate for the subsequent cycles. All tests were performed using the block sitting upright; that is in the manner they would be loaded in construction with the cylindrical voids vertical.

\subsubsection{Measurement of Strain}

The strain values reported below are simply the overall deflection of the block instead of localized strains measured with strain gauges. After yielding, the blocks began to bulge or ripple in failure planes. The location and size of these bulges appeared to be dependent on how the straw was placed in the mold during the manufacturing process. 
Because of this uneven deformation over the entire volume of the blocks, the overall strain measured from the test machine head displacement was used. This bulging phenomenon can be seen in Figure 3-2.

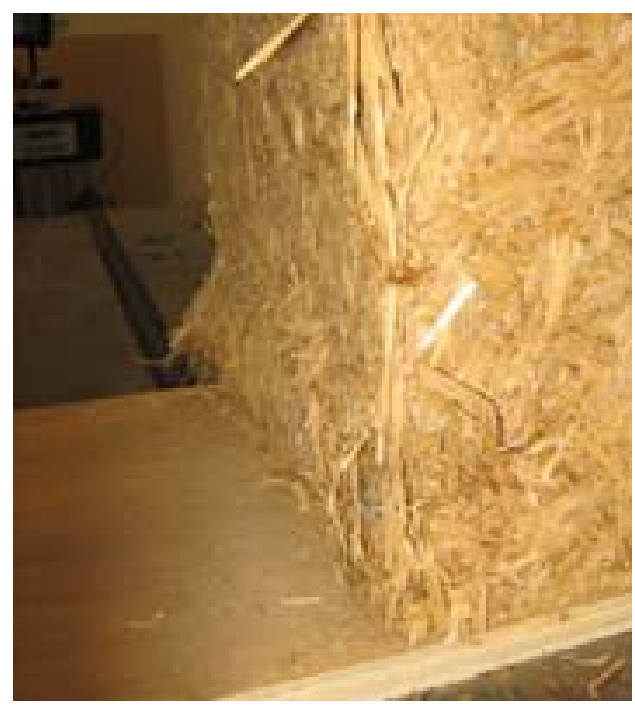

Figure 3-2: Typical Failure at Higher Strains

\subsubsection{Testing Program Terminology}

In the following report, various terms will be used repeatedly to describe phenomena observed in the compressive testing of the straw blocks. First, all of the following strength values have the subscript "gross" or "g”. This is referring to the gross area of a tested block specimen. Although the blocks are 12 inches by 24 inches (288 square inches), each block has two 4 inch diameter cylindrical voids. Each block actually has a cross sectional area of 262.9 square inches after subtracting out these voids. So, on a material level, the strengths reported below could be increased 9.5\% to properly describe the strength of the straw with binding agent.

The reason the gross area was used is twofold: 1) for simplicity in future calculations. The wall is exactly 12 inches thick, and in calculations the structural 
demand on a wall as well as its strength are typically given in pounds per lineal foot. Therefore, loads reported can be easily converted to wall strength per lineal foot. 2) it is actually the gross area that would be physically loaded. Although the straw and binding agent may have a "net” strength, the entire block used in a hypothetical wall assembly would carry load over its “gross” area, voids included.

Another important definition comes from the stress-strain behavior observed in the tested blocks. Each block was found to have an initial "toe" where voids within the block are closing up, and settlement is occurring on a material level. During this phase, the material exhibits strain-hardening behavior until it reaches a linear elastic zone. This linear elastic zone was observed for all specimens and is where the elastic modulus was determined from the slope of the stress-strain curve. Using Microsoft Excel's linear regression tools, the slope of this linear region was determined. This slope represents the elastic modulus of that sample in pounds per square inch. An example linear approximation of Young's modulus (in this case, 877 psi) is found in Figure 3-3.

The sample eventually starts to soften at higher strains and this point where the elastic modulus deviates from the linear region will be called the "proportional limit”. This proportional limit represents the yielding of the straw block in that after this point, the stiffness is drastically reduced. These definitions are represented in Figure 3-4 in an example stress-strain plot. 


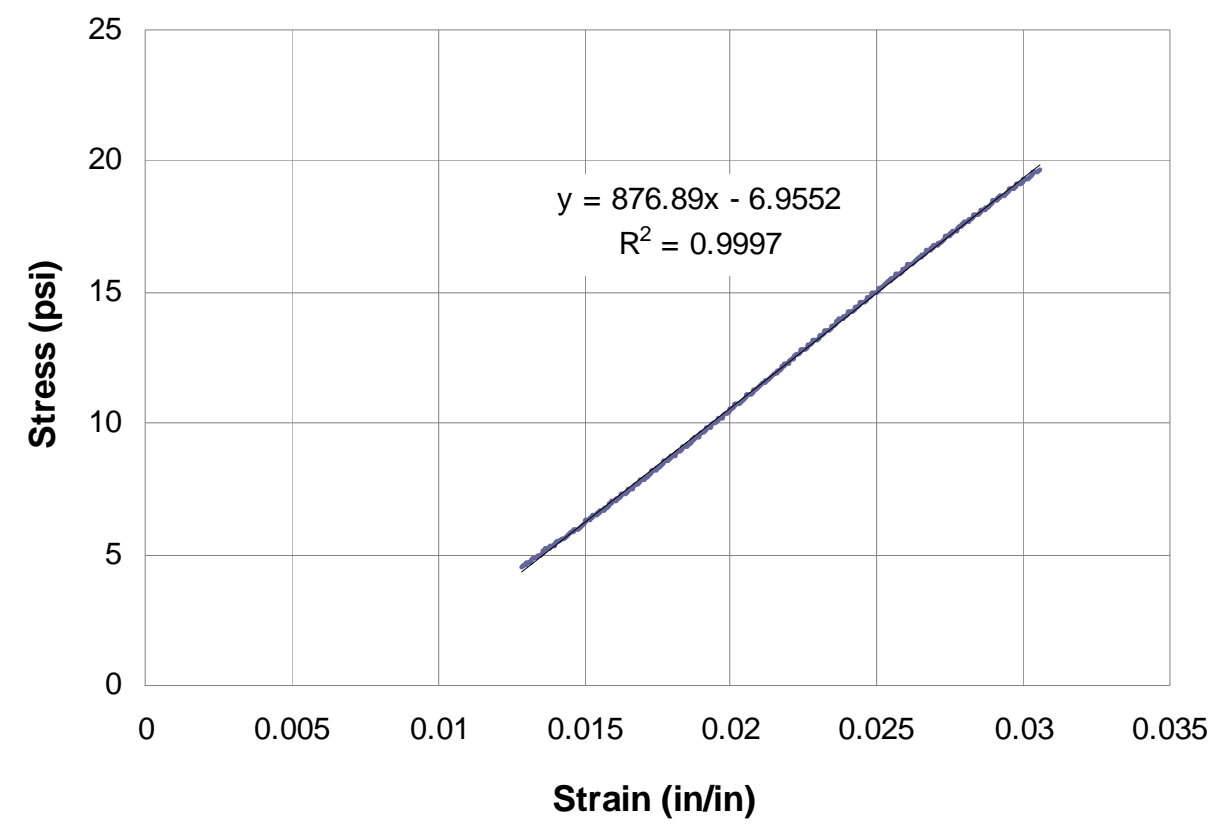

Figure 3-3: Elastic Modulus Approximation

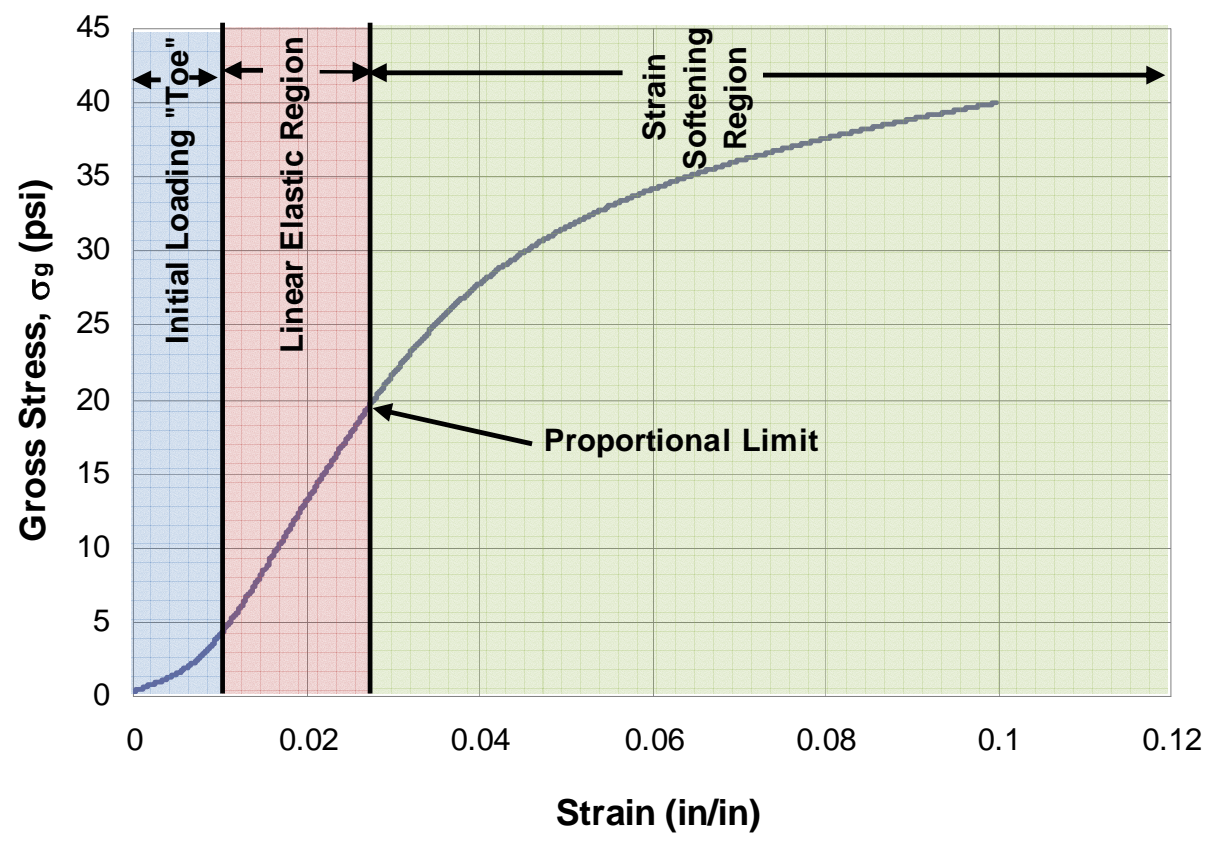

Figure 3-4: Stress-Strain Behavioral Definitions 


\subsubsection{Compression Testing Protocol}

Compressive testing was performed continuously throughout the project; from the early stages of determining construction methods to the investigation of blocks used in shear wall testing. The complete tested sample set is summarized in Table 3-1.

\section{Table 3-1: Summary of Compression Testing Program}

\begin{tabular}{cc} 
Specimen & Description \\
\hline \hline $14 \_1$ & Prior Testing \\
$14 C \_1$ & Prior Testing \\
$15 \_1$ & Prior Testing \\
15C_1 & Prior Testing \\
full01 & 3 cycles to $10 \%$ strain \\
half02A & 5 cycles to $5 \%$ strain \\
half03A & 5 cycles to 5\% followed by 1 cycle to 10\% \\
half02B & 5 cycles to proportional limit followed by 1 cycle to 10\% \\
half03B & 4 cycles to proportional limit \\
half04D & 5 cycles to proportional limit followed by 4 cycles to 10\% strain \\
half04C & 5 cycles to toe followed by 1 cycle to proportional limit \\
wall1_D3 & After Shear Wall Testing \\
wall1_E2 & After Shear Wall Testing \\
wall2_A4 & After Shear Wall Testing \\
wall2_C3 & After Shear Wall Testing \\
wall2_H5 & After Shear Wall Testing \\
wall3_A4 & After Shear Wall Testing \\
wall3_H5 & After Shear Wall Testing \\
wall4_D2 & After Shear Wall Testing \\
wall4_H5 & After Shear Wall Testing
\end{tabular}

\subsubsection{Prior Block Testing}

Prior testing was performed at Cal Poly by Dr. Linda Vanasupa to determine the optimum amount of binding agent used in making the blocks while balancing cost and strength. The samples here forth referred to as “14_1”, “14c_1”, “15_1” and “15c_1” are blocks that performed best and became the prototypical mixture moving forward with the development of this product. 
In order to ensure that the blocks delivered for the purposes of this report were comparable to the prototypes, a test was performed replicating the prior tests. The specimen was loaded to $10 \%$ strain (1.2 inches of deflection) and then unloaded. The specimen Full01 exhibited properties within an acceptable range of the original prototype blocks. The results of the prior tests and this first proofing test are found in Table 3-2.

Table 3-2: Comparison to Prior Block Testing

\begin{tabular}{cccc} 
& \multicolumn{2}{c}{$\sigma_{\text {g,prop }}$} & $E_{g}$ \\
\cline { 2 - 3 } Specimen & $(\mathrm{psi})$ & $(\mathrm{psf})$ & $(\mathrm{psi})$ \\
\hline \hline 14_1 & 25.0 & 3602 & 841 \\
14c_1 & 19.6 & 2828 & 877 \\
15_1 & 20.5 & 2946 & 1049 \\
15c_1 & 22.1 & 3183 & 967 \\
\hline Prior Avg. & 21.8 & 3140 & 934 \\
full01 & 20.9 & 3004 & 909 \\
\hline Difference & $4.5 \%$ & $4.5 \%$ & $2.7 \%$
\end{tabular}

\subsubsection{Comparison of Full Blocks to Half Blocks}

Another issue with the materials investigation was the limited availability of the straw blocks due to manufacturing time and cost. It would be more efficient and less wasteful to use partial blocks to perform the litany of tests in the program. Also, in construction, blocks would have to be cut in half and modified and if this adversely effected the material properties, this could cause and issue with weaker blocks within stronger, full blocks. Two blocks were sawed in half and the halves were tested in a similar manner to the prototype blocks and Full01. Figure 3-5 shows the results of these tests up to their respective proportional limits. 


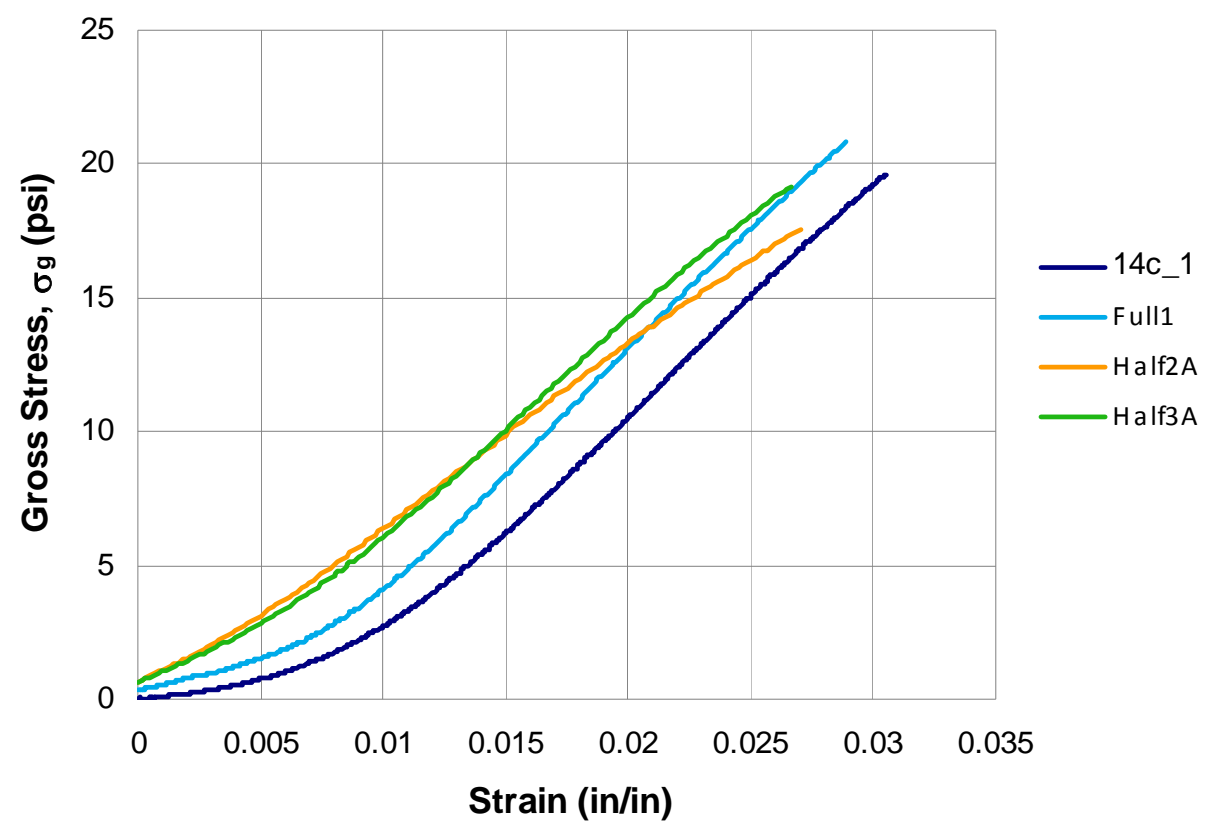

Figure 3-5: Comparison of Half to Full Block Strengths

A slight decrease in stiffness and yield strength can be seen in the half blocks.

Also, there appears to be a reduction in the amount of strain needed to get past the initial toe. Both of these factors could be caused by the damage that comes with the sawing process. Two methods were used to split the blocks in half: using a hand saw to cut through the entire 12 inch block and using a table saw. The table saw produced a cleaner and more even cut and is recommended for future construction.

Another phenomenon that occurred in testing half blocks was an obvious differential displacement between the two sides (cut and uncut). Even though the test machine head was centered on the block, the sawed side would deflect less than the untouched side. The explanation for this is that the blocks are not homogenous with respect to the straw and binding agent density. The center, or core, of the block that was sawed through appears to be the stiffer portion of the block when compared to the external surfaces. While there may not be a solution to this byproduct of the 
manufacturing process, it does not appear to have too large of an effect on overall block performance. Table 3-3 summarizes all tested half and whole blocks in the entire program. The parameter of strength at $21 / 2 \%$ strain was included to account for the fact that the half blocks have a shorter toe region. Therefore under 0.3 inches of deflection, a half block will hold approximately as much as a whole one would.

Table 3-3: Comparison of Half to Whole Blocks

\begin{tabular}{|c|c|c|c|c|}
\hline \multirow{2}{*}{$\begin{array}{l}\text { Specimen } \\
\text { (\# Tested) }\end{array}$} & \multicolumn{2}{|c|}{$\sigma_{\text {g,prop }}$} & \multirow{2}{*}{$\begin{array}{c}E_{g} \\
(p s i) \\
\end{array}$} & \multirow{2}{*}{$\begin{array}{c}\sigma_{\mathrm{g}, 2.5 \%} \\
(\mathrm{psi}) \\
\end{array}$} \\
\hline & (psi) & (psf) & & \\
\hline full (11) & 23.4 & 3365 & 800 & 14.4 \\
\hline half (8) & 18.4 & 2651 & 686 & 14.7 \\
\hline Difference & $26.9 \%$ & $26.9 \%$ & $16.5 \%$ & $-1.9 \%$ \\
\hline
\end{tabular}

\subsubsection{Relation of Strength to Density}

Throughout the research into material properties of straw bale, much attention has been paid to the relationship between density and stiffness and strength. Bou-Ali (1993) reported a positive correlation between the density of a bale and its ultimate strength. Stephens and Budinger (2000) showed that their “supercompressed” bales (high density) exhibited much stiffer and stronger behavior than normal bales.

The straw blocks being investigated in this report tend to not vary as much in density and composition due to the more controlled manufacturing process. Yet, the slight variations in density can be attributed to this still developing process which includes human error and rough estimations of proportions. Prior to testing, the majority of the specimens were weighed and tabulated. In Figure 3-6 below, the density of the straw blocks is compared to the measured elastic moduli. 


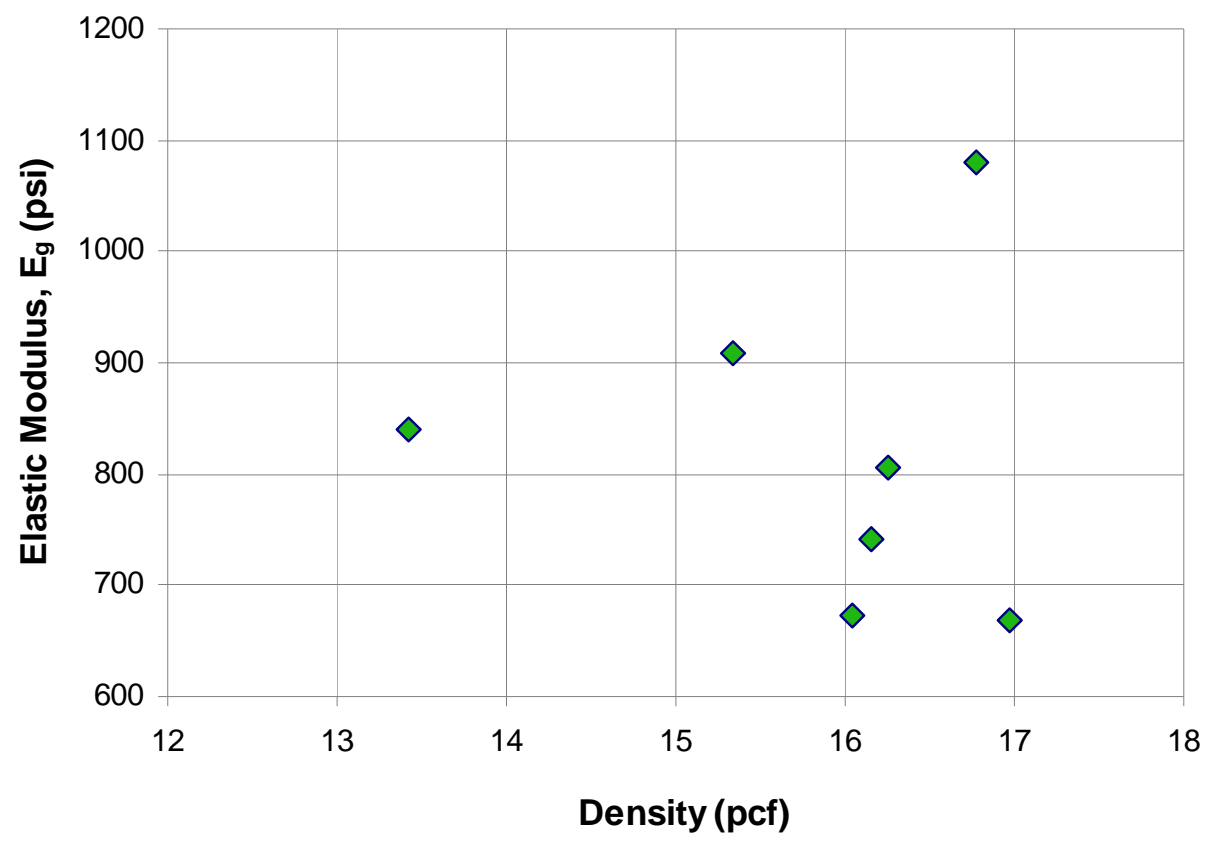

Figure 3-6: Density versus Elastic Moduli

No clear correlation can be seen in the data presented above. If anything, it appears that the four specimens with the lowest stiffnesses are on the upper end of the density measurements but still the data does not exhibit an identifiable trend. It should be noted that the straw blocks tested have measured densities approximately twice that of normal straw bales (15-17 pcf versus 7-8 pcf). Yet the range of measured elastic moduli of the straw blocks are four to ten times higher than the bales determined from Bou-Ali's (1993) testing program (700-1100 psi versus 60-200 psi).

Density variability within blocks produced right after each other in the same conditions can be attributed to the human measurement error as discussed earlier. Stiffness has been shown to be relatively unaffected by these variations. Figures 3-7 and 3-8 show the relationship between strength and density and the strain at which the linear elastic range begins and density, respectively. 


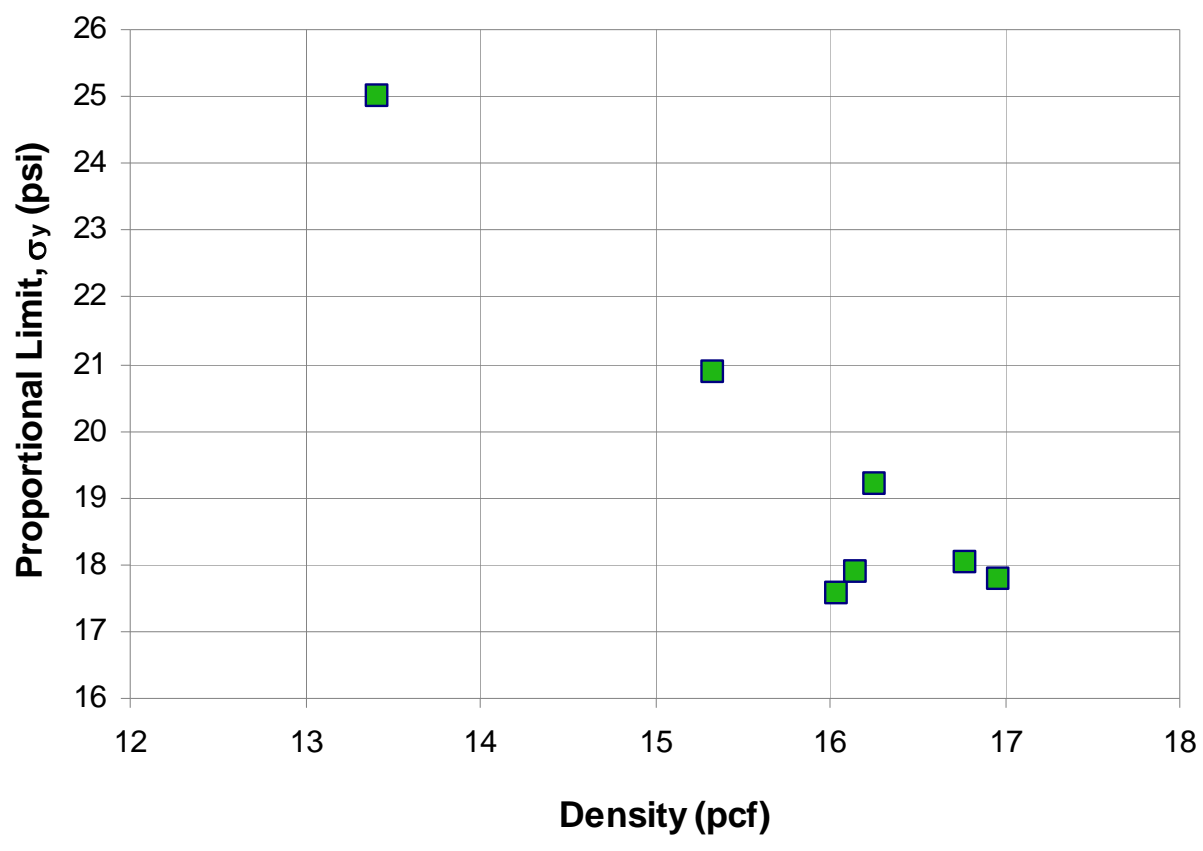

Figure 3-7: Density versus Proportional Limits

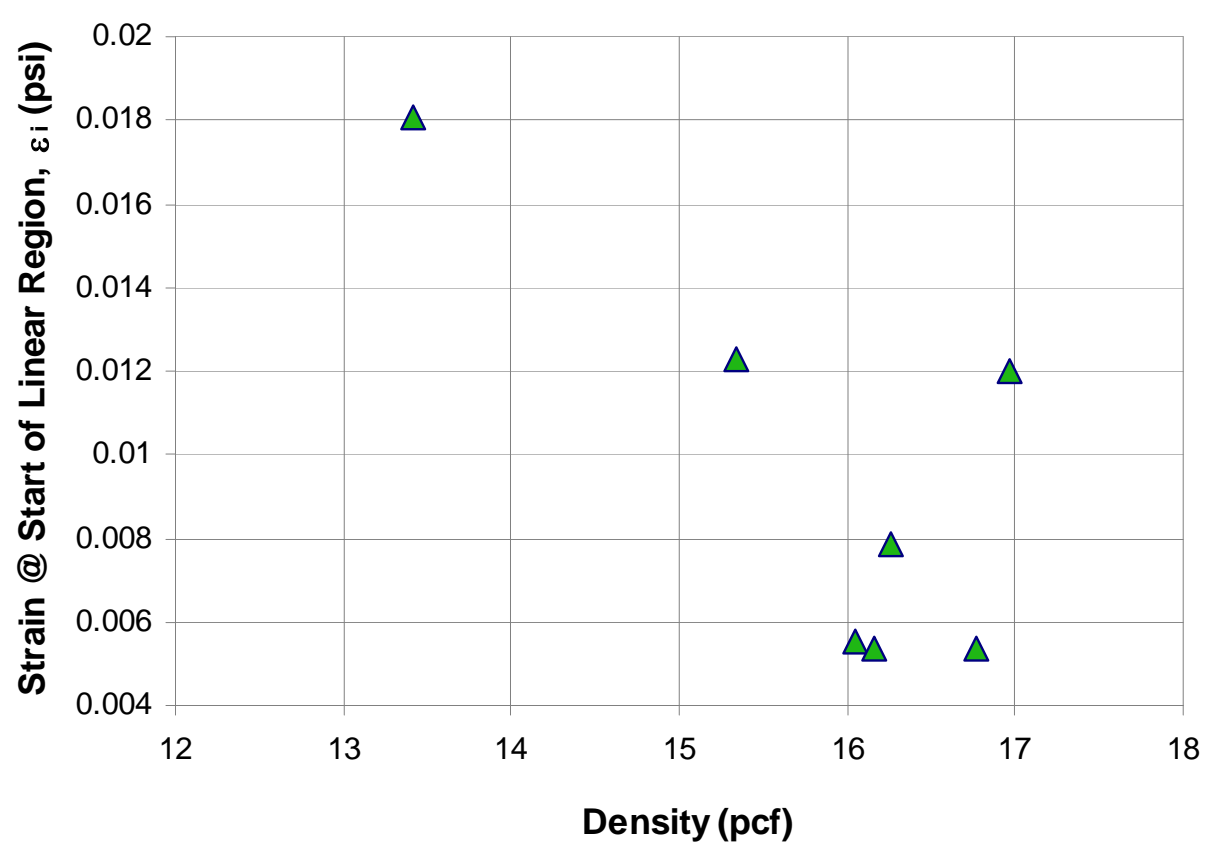

Figure 3-8: Density versus Initial Strains

Figure 3-7 and 3-8 both show clear negative correlations between density and strength and initial strain. While it may be counterintuitive, the denser a straw block is, 
the lower its yield or proportional limit will tend to be. Since the weight of the overall block is largely the straw itself with the binding agent only adding a minute amount of mass, it does not appear that more straw is better. Instead it may be a factor that is harder to quantify and measure such as straw or culm orientation within the block. The straw is placed in clumps into the mold and these clumps can be obviously seen when cutting a block in half. It is possible that the denser a straw block becomes in the mold, the more straw gets pressed flat and not in the stronger, vertical direction.

The relationship between initial strain and density makes logical sense in its negative correlation. The denser a straw block is, the lower strain value is needed to reach the linear elastic portion of the stress-strain behavior. This can be attributed to a lesser amount of void space within the denser blocks that have to close and settle in the toe region. So although a denser block may have a lower yield limit, at lower strains it may actually be stronger than a less dense block in that the softer toe region is avoided.

One issue of note about the density data presented above is the outlier point with a density of approximately 13.4 pcf. This data point represents the only one of the blocks from prior testing (specimen 14) that had density data recorded at the time. Even though this prototype block was used in developing mixing proportions, it appears that the blocks produced at that time were less dense. This could be caused by different straw sources or just the fact that an exact match in mix proportions was not used for the blocks in this study. Disregarding this point or not, a negative correlation in Figures 3-7 and 3-8 can still be seen in the recorded data. 


\subsubsection{Cyclic Testing of Blocks}

Cyclic testing was a major facet of this materials investigation because the second portion of the testing included shear wall testing in which the blocks would be loaded and reloaded. The specimen Full01 was loaded once to $10 \%$ strain as mentioned above, and in order to investigate this cyclic behavior, was displaced to $10 \%$ strain twice more immediately after. The results from this experiment are shown in Figure 3-9 and Table 34.

Table 3-4: Full01 Specimen Results

\begin{tabular}{ccccccc} 
cycle & $\begin{array}{c}\varepsilon_{\text {resid }} \\
(\mathrm{in} / \mathrm{in})\end{array}$ & $\begin{array}{c}\text { Change } \\
(\%)\end{array}$ & $\begin{array}{c}\sigma_{\mathrm{g}, \text { prop }} \\
(\mathrm{psi})\end{array}$ & $\begin{array}{c}\sigma_{\mathrm{g}, \mathrm{prop}} \\
(\mathrm{psf})\end{array}$ & $\begin{array}{c}\mathrm{E}_{\mathrm{g}} \\
(\mathrm{psi})\end{array}$ & $\begin{array}{c}\text { Change } \\
(\%)\end{array}$ \\
\hline \hline 1 & 0.0216 & -- & 20.9 & 3004 & 909 & -- \\
2 & 0.0236 & 9.3 & 28.6 & 4113 & 497 & -45.7 \\
3 & 0.0249 & 5.6 & 21.6 & 3107 & 460 & -7.4
\end{tabular}

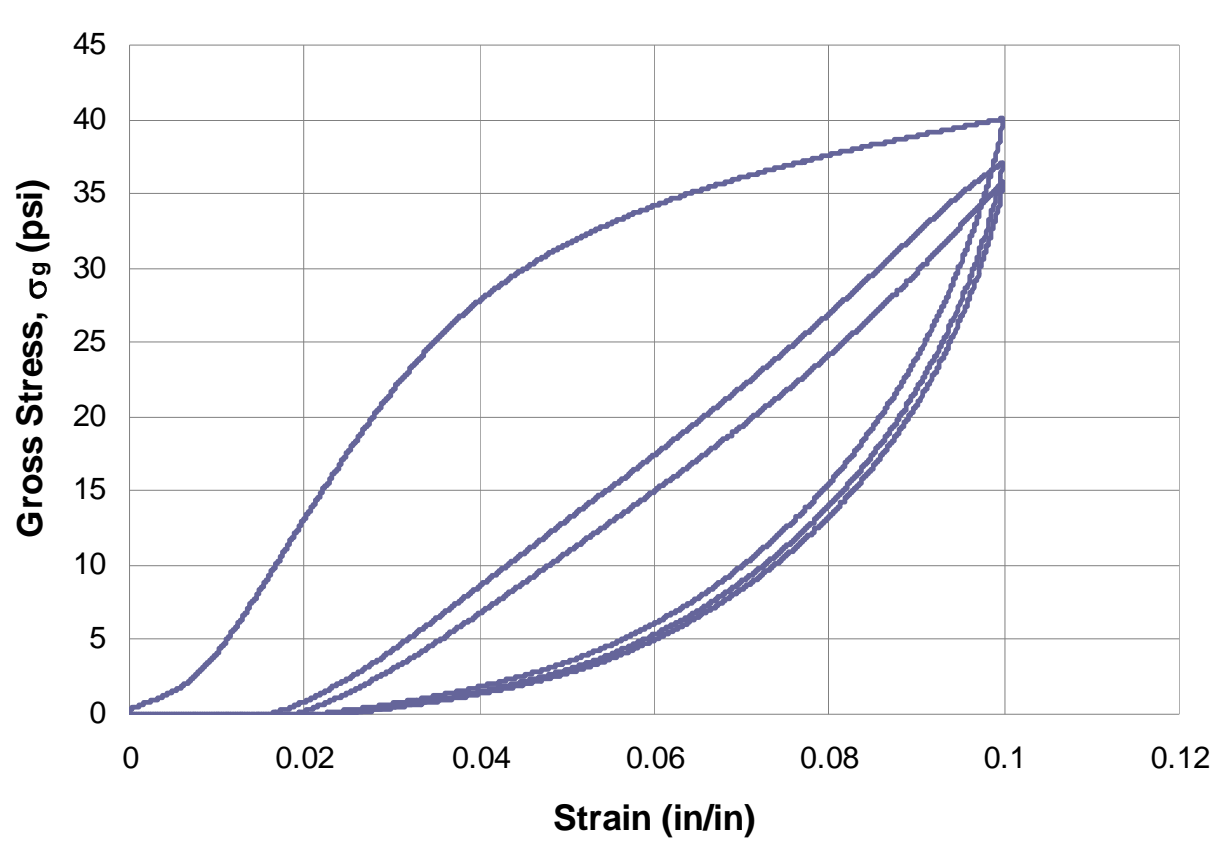

Figure 3-9: Full01 Specimen Results 
It can be seen that a loading the specimen far past its yield point and causes considerable damage on a material level. The residual strain values of approximately $2 \%$ show that the initial settlement in the toe region is finished once the specimen is loaded up to $10 \%$ strain. While the proportional limits do not reduce in subsequent cycles (in fact the largest was found in the second cycle), Table 3-3 and Figure 3-9 both show a significant reduction (nearly one half) in the elastic modulus of the sample.

Cycling to a smaller strain level, such as 5\% would produce less damage on a material level. The results from cycling the specimen Half02A to this lower strain five times are found in Table 3-5 and Figure 3-10.

Table 3-5: Half02A Specimen Results

\begin{tabular}{cccccccc}
$\varepsilon_{\text {resid }}$ & $\begin{array}{c}\text { Change } \\
(\%)\end{array}$ & $\begin{array}{c}\sigma_{\mathrm{g}, \varepsilon 5 \%} \\
(\mathrm{psi})\end{array}$ & $\begin{array}{c}\sigma_{\mathrm{g}, \varepsilon 5 \%} \\
(\mathrm{psf})\end{array}$ & $\begin{array}{c}\text { Change } \\
(\%)\end{array}$ & $\begin{array}{c}\mathrm{E}_{\mathrm{g}} \\
(\mathrm{psi})\end{array}$ & $\begin{array}{c}\text { Change } \\
(\%)\end{array}$ \\
\hline \hline 1 & 0.0077 & -- & 27.8 & 4004 & -- & 673 & -- \\
2 & 0.0087 & 13.2 & 26.8 & 3853 & -3.8 & 642 & -4.5 \\
3 & 0.0095 & 9.4 & 26.2 & 3768 & -2.2 & 737 & 14.7 \\
4 & 0.0100 & 5.5 & 25.7 & 3702 & -1.8 & 743 & 0.8 \\
5 & 0.0105 & 5.0 & 25.4 & 3657 & -1.2 & 677 & -8.8
\end{tabular}




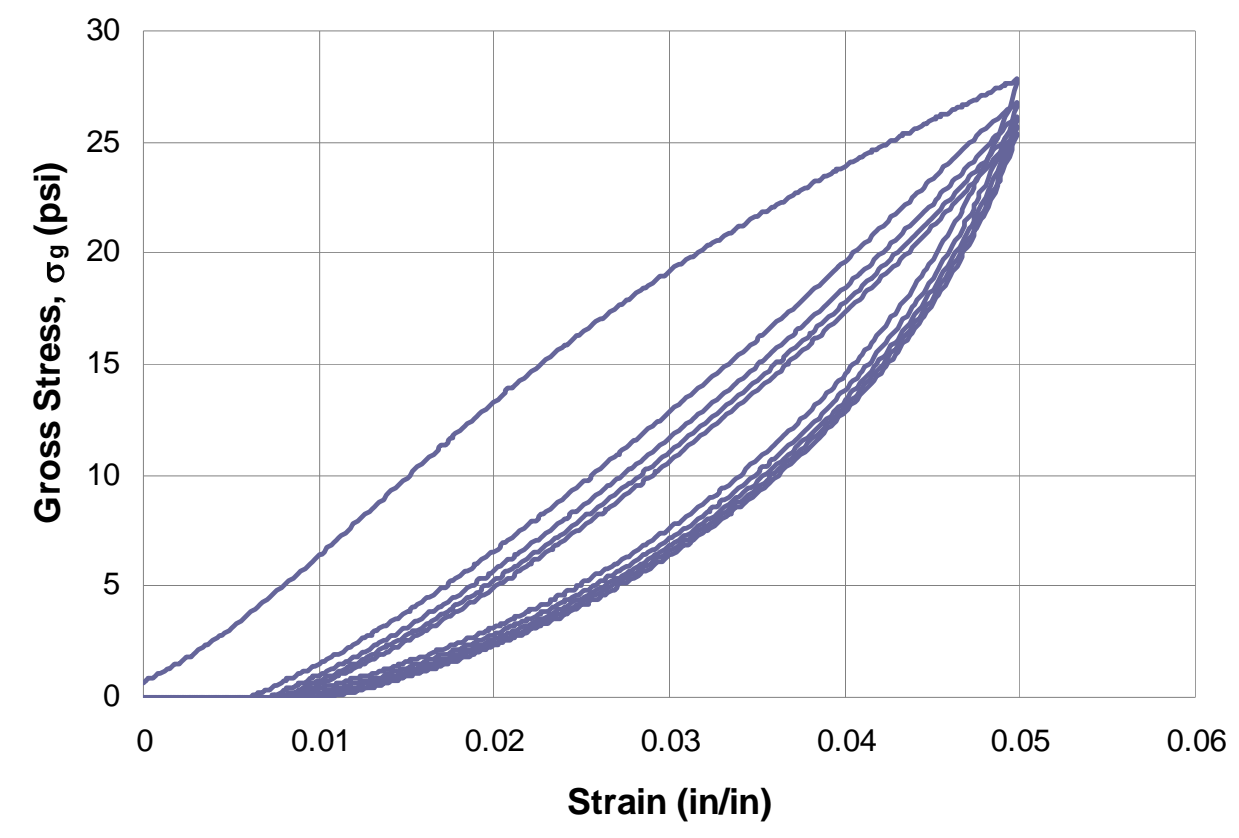

Figure 3-10: Half02A Specimen Results

It can be seen that the lower strain value does not damage the specimen nearly as much. This is evidenced by the lower residual strain values in between cycles (about 0.01 versus $0.02 \mathrm{in} / \mathrm{in})$. Also, cycling at $5 \%$ strain accomplishes reducing the effects of the initial settlement toe. The data above also shows that in each subsequent cycle, the stress at 5\% strain asymptotically approaches a value of 3,600 psi. Unlike the test cycling to $10 \%$, the elastic moduli do not drop after each cycle; they even substantially increase after two cycles.

The next test on specimen Half03A was similar to Half02A in the sense that the sample was cycled five times to $5 \%$ strain. In this test though, after the fifth cycle, the test machine was run out to $10 \%$ strain. The results are shown in Table 3-6 and Figure 311. 
Table 3-6: Half03A Specimen Results

\begin{tabular}{cccccccc} 
cycle & $\begin{array}{c}\varepsilon_{\text {resid }} \\
(\mathrm{in} / \mathrm{in})\end{array}$ & $\begin{array}{c}\text { Change } \\
(\%)\end{array}$ & $\begin{array}{c}\sigma_{\mathrm{g}, 55 \%} \\
(\mathrm{psi})\end{array}$ & $\begin{array}{c}\sigma_{\mathrm{g}, 55 \%} \\
(\mathrm{psf})\end{array}$ & $\begin{array}{c}\text { Change } \\
(\%)\end{array}$ & $\begin{array}{c}\mathrm{E}_{\mathrm{g}} \\
(\mathrm{psi})\end{array}$ & $\begin{array}{c}\text { Change } \\
(\%)\end{array}$ \\
\hline \hline 1 & 0.0083 & -- & 28.7 & 4137 & -- & 805 & -- \\
2 & 0.0104 & 26.2 & 27.5 & 3962 & -4.2 & 707 & -12.2 \\
3 & 0.0113 & 8.0 & 26.7 & 3848 & -2.9 & 698 & -1.2 \\
4 & 0.0116 & 3.0 & 26.2 & 3777 & -1.8 & 694 & -0.6 \\
5 & 0.0120 & 3.3 & 25.8 & 3717 & -1.6 & 780 & 12.3 \\
6 & 0.0287 & 139.6 & 39.0 & 5613 & 51.0 & 671 & -14.0
\end{tabular}

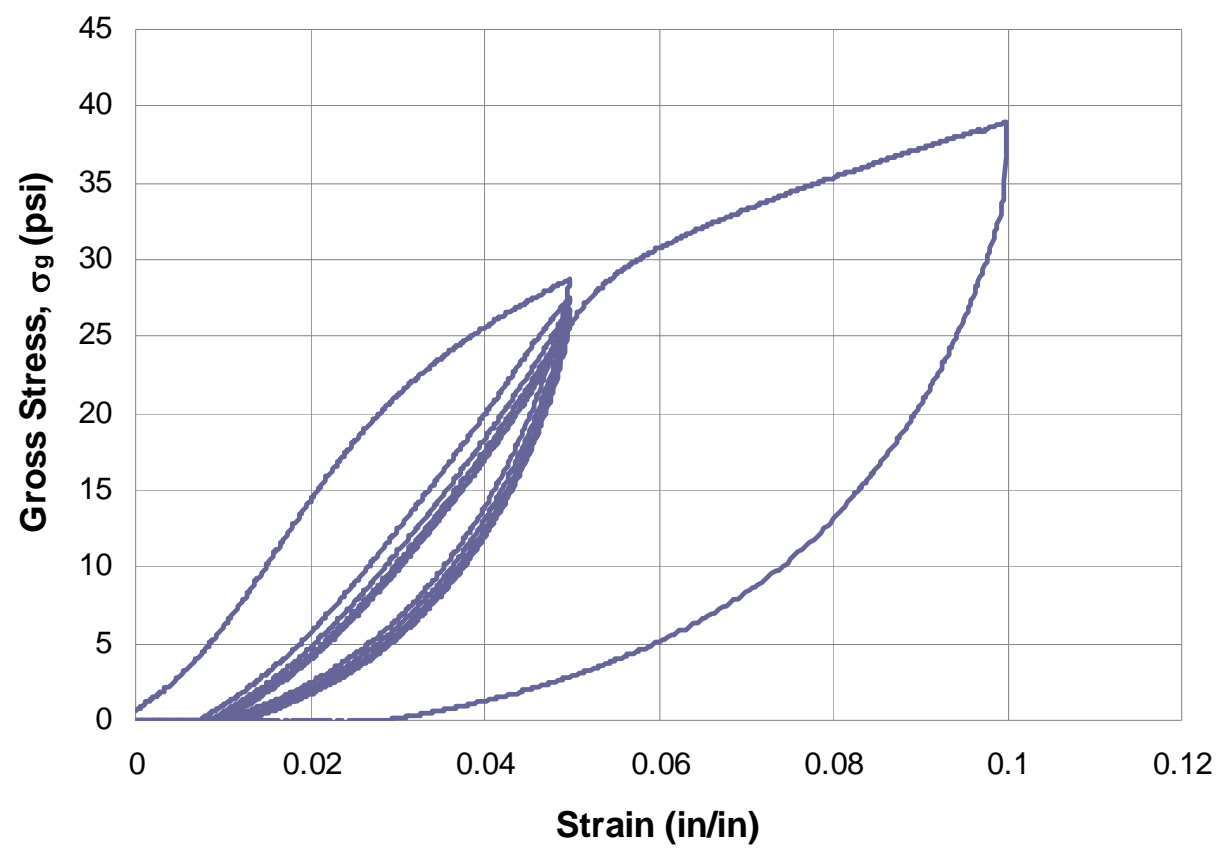

Figure 3-11: Half03A Specimen Results

In this test, the cycle out to $10 \%$ strain produced a residual strain that was more than twice as high as after the five leading cycles. This is to be expected with the damage that occurs at strains as high as $10 \%$. Also, even though the elastic moduli are fluctuating between cycles as before, after the first cycle, minor strain-hardening behavior can be seen in the following cycles. It also should be noted that the unloading curve after the 
$10 \%$ strain cycle does not follow the earlier curves. This is because of the increased amount of energy dissipated in the form of bulging damage.

After a series of tests had been performed, an average proportional limit of approximately 2,600 psf (18.1 psi) was determined. This target stress was used in the testing of specimen Half02B. In this test, the block was loaded five times to the assumed proportional limit and then once to $10 \%$ strain. The results are shown in Table 3-7 and Figure 3-12 and 3-13.

Table 3-7: Half02B Specimen Results

\begin{tabular}{ccccccc} 
cycle & $\begin{array}{c}\varepsilon_{\text {resid }} \\
(\text { in/in })\end{array}$ & $\begin{array}{c}\text { Change } \\
(\%)\end{array}$ & $\begin{array}{c}\sigma_{\mathrm{g}, \text { prop }} \\
(\mathrm{psi})\end{array}$ & $\begin{array}{c}\sigma_{\mathrm{g}, \text { prop }} \\
(\mathrm{psf})\end{array}$ & $\begin{array}{c}\mathrm{E}_{\mathrm{g}} \\
(\mathrm{psi})\end{array}$ & $\begin{array}{c}\text { Change } \\
(\%)\end{array}$ \\
\hline \hline 1 & 0.0073 & -- & 17.0 & 2451 & 669 & -- \\
2 & 0.0087 & 19.5 & 17.6 & 2537 & 851 & 27.3 \\
3 & 0.0094 & 8.9 & 17.7 & 2542 & 867 & 1.9 \\
4 & 0.0102 & 7.5 & 17.7 & 2546 & 900 & 3.8 \\
5 & 0.0110 & 8.5 & 17.9 & 2577 & 908 & 0.8 \\
6 & 0.0250 & 126.7 & 21.0 & 3023 & 959 & 5.7
\end{tabular}

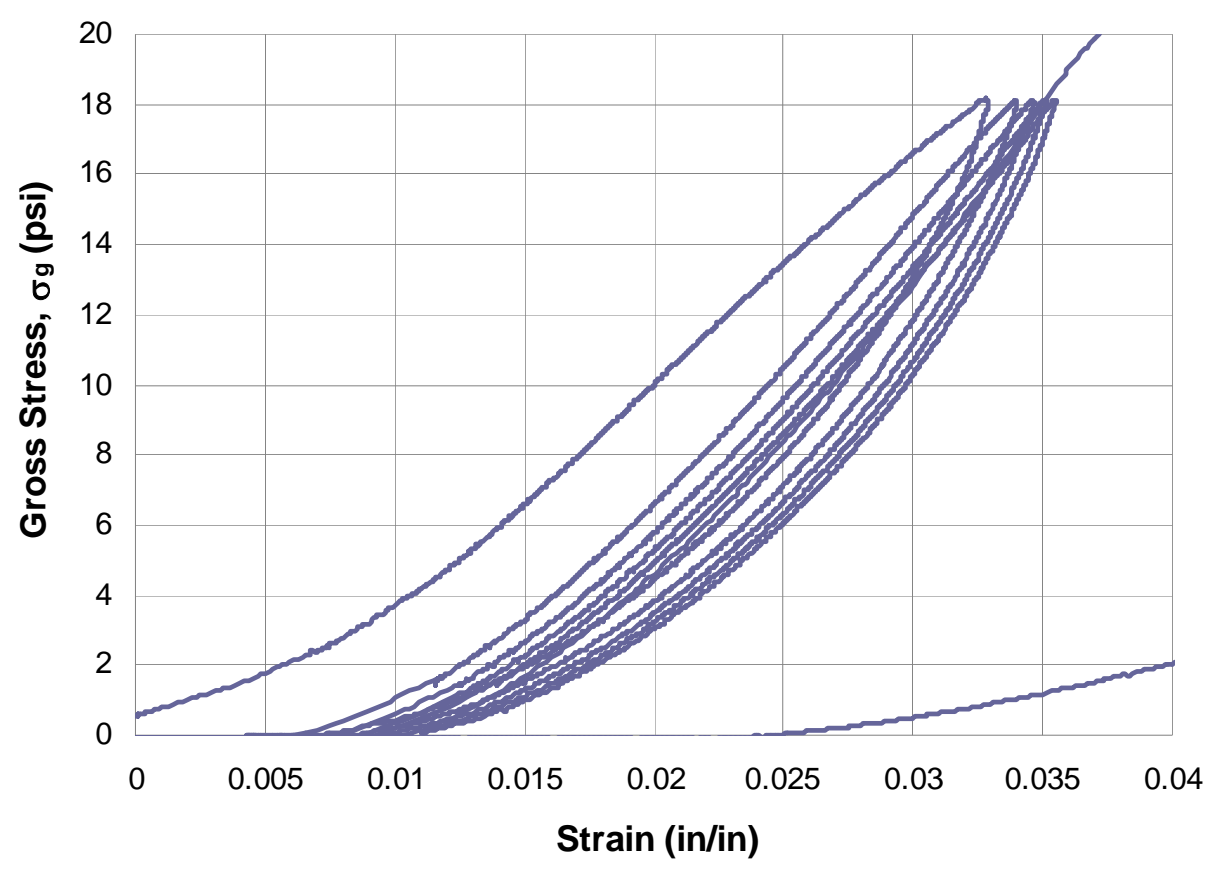

Figure 3-12: Half02B Proportional Limit Cycles 


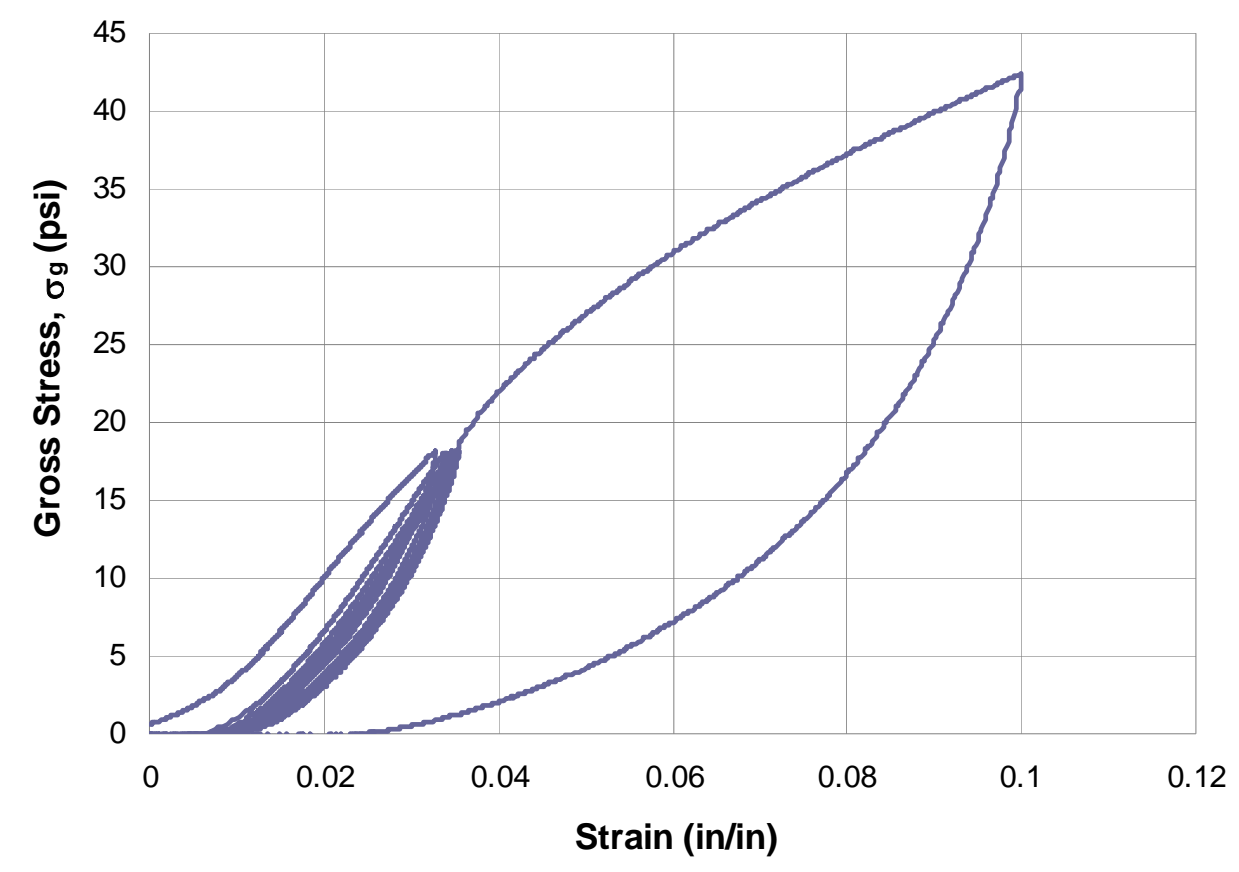

Figure 3-13: Half02B Specimen Results

Even though the target in this test was a specified stress, the strains reached in the first five cycles were much lower than Half03A. Because of this, the damage that typically occurs after the yield point did not occur and there is actually stiffening that occurs after the first cycle. The elastic modulus increased from 669 to 851 psi and then continued to increase with each succeeding cycle. This could be attributed to voids and irregularities being worked out each cycle as the specimen remained within the linear elastic range until the very last cycle to $10 \%$ strain.

Specimen Half03B was to be loaded in a similar manner but then cycled up at $10 \%$, but the test machine overheated after four cycles up to the proportional limit. The same phenomenon of increasing elastic moduli can be seen in the data in Table 3-8. In this case, the residual strain was much lower than Half02B even though both were loaded 
the same. This could be attributed to the fact that each specimen came from different blocks with different densities and compositions.

Table 3-8: Half03B Specimen Results

\begin{tabular}{ccccccc} 
cycle & $\begin{array}{c}\varepsilon_{\text {resid }} \\
(\mathrm{in} / \mathrm{in})\end{array}$ & $\begin{array}{c}\text { Change } \\
(\%)\end{array}$ & $\begin{array}{c}\sigma_{\mathrm{g}, \text { prop }} \\
(\mathrm{psi})\end{array}$ & $\begin{array}{c}\sigma_{\text {g,prop }} \\
(\mathrm{psf})\end{array}$ & $\begin{array}{c}\mathrm{E}_{\mathrm{g}} \\
(\mathrm{psi})\end{array}$ & $\begin{array}{c}\text { Change } \\
(\%)\end{array}$ \\
\hline \hline 1 & 0.0024 & -- & 17.9 & 2578 & 742 & -- \\
2 & 0.0030 & 24.7 & 17.9 & 2575 & 899 & 21.1 \\
3 & 0.0033 & 12.3 & 16.7 & 2409 & 890 & -0.9 \\
4 & 0.0011 & -67.6 & 17.6 & 2529 & 913 & 2.6
\end{tabular}

An additional cyclic test was performed on specimen Half04D after the machine overheated for Half03B. The results of this test can be seen in Table 3-9 and Figure 3-14.

Table 3-9: Half04D Specimen Results

\begin{tabular}{ccccccc} 
cycle & $\begin{array}{c}\varepsilon_{\text {resid }} \\
(\mathrm{in} / \mathrm{in})\end{array}$ & $\begin{array}{c}\text { Change } \\
(\%)\end{array}$ & $\begin{array}{c}\sigma_{\mathrm{g}, \text { prop }} \\
(\mathrm{psi})\end{array}$ & $\begin{array}{c}\sigma_{\mathrm{g}, \mathrm{prop}} \\
(\mathrm{psf})\end{array}$ & $\begin{array}{c}\mathrm{E}_{\mathrm{g}} \\
(\mathrm{psi})\end{array}$ & $\begin{array}{c}\text { Change } \\
(\%)\end{array}$ \\
\hline \hline 1 & 0.0015 & -- & 18.0 & 2599 & 1079 & -- \\
2 & 0.0020 & 34.9 & 18.0 & 2593 & 1268 & 17.5 \\
3 & 0.0022 & 11.8 & 17.4 & 2512 & 1258 & -0.8 \\
4 & 0.0021 & -6.8 & 17.2 & 2478 & 1232 & -2.1 \\
5 & 0.0023 & 10.7 & 17.7 & 2544 & 1276 & 3.6 \\
6 & 0.0234 & 922.5 & 19.6 & 2818 & 1254 & -1.7 \\
7 & 0.0267 & 14.2 & 25.3 & 3648 & 467 & -62.8 \\
8 & 0.0281 & 5.0 & 22.3 & 3208 & 489 & 4.6 \\
9 & 0.0294 & 4.8 & 17.3 & 2485 & 412 & -15.7
\end{tabular}




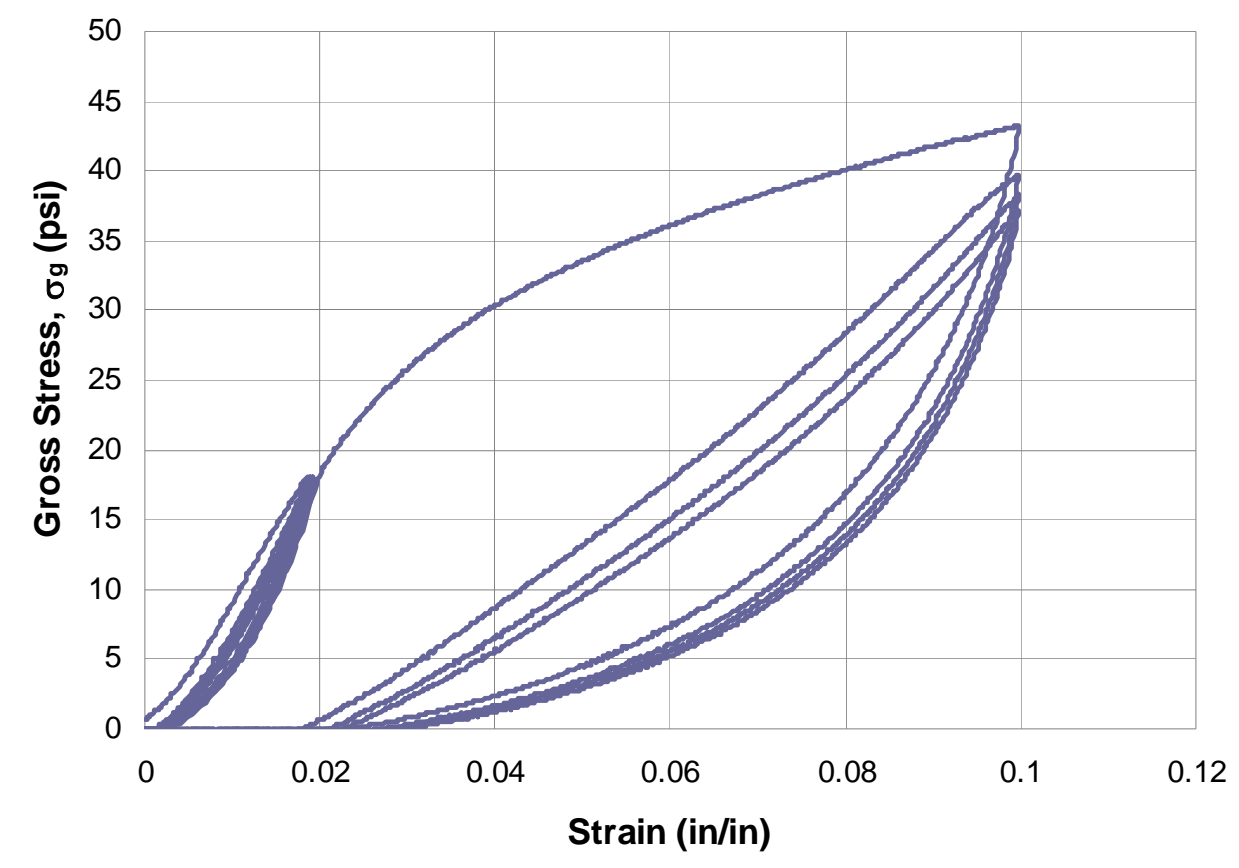

Figure 3-14: Half04D Specimen Results

This particular specimen was among the strongest tested of the program. After the first cycle to the proportional limit, the elastic modulus had the large increase as seen before. Cycling out at $10 \%$ strain lead to significant damage of the block and the elastic modulus dropped by nearly two-thirds. This just further illustrates how important staying within the linear elastic range is in the usage of these straw blocks. The drastic strainsoftening that occurs after the yield point represents permanent damage and large residual strains. While this behavior is not isolated to straw blocks, it is an important factor that must be considered in designing with this material.

\subsubsection{Preliminary Compression Testing Results}

As with any manufactured or natural material, there will be variation in measured properties such as strength and stiffness. The materials study produced average values that would be used later in this report for computer modeling and prediction of structural 
behavior of an entire wall system. These values are tabulated below in Table 3-10 and the disparities in toe size, linear elastic slope, and proportional limits are shown graphically in Figure 3-15.

Table 3-10: Summary of Preliminary Material Testing Results

\begin{tabular}{cccccccc} 
specimen & $\begin{array}{c}\varepsilon_{\text {init }} \\
(\mathrm{in} / \mathrm{in})\end{array}$ & $\begin{array}{c}\sigma_{\mathrm{g}, \text { init }} \\
(\mathrm{psi})\end{array}$ & $\begin{array}{c}\sigma_{\mathrm{g}, \text { init }} \\
(\mathrm{psf})\end{array}$ & $\begin{array}{c}\sigma_{\mathrm{g}, \text { prop }} \\
(\mathrm{psi})\end{array}$ & $\begin{array}{c}\sigma_{\mathrm{g}, \text { prop }} \\
(\mathrm{psf})\end{array}$ & $\begin{array}{c}\mathrm{E}_{\mathrm{g}} \\
(\mathrm{psi})\end{array}$ & $\begin{array}{c}\rho \\
(\mathrm{pcf})\end{array}$ \\
\hline \hline 14_1 & 0.0181 & 5.3 & 756.6 & 25.0 & 3602 & 841 & 13.4 \\
14c_1 & 0.0129 & 4.5 & 654.1 & 19.6 & 2828 & 877 & -- \\
15_1 & 0.0091 & 3.5 & 504.7 & 20.5 & 2946 & 1049 & -- \\
15c_1 & 0.0184 & 5.8 & 830.2 & 22.1 & 3183 & 967 & -- \\
full01 & 0.0123 & 5.9 & 848.0 & 20.9 & 3004 & 909 & 15.3 \\
half02A & 0.0055 & 3.4 & 495.0 & 17.6 & 2530 & 673 & 16.0 \\
half03A & 0.0078 & 4.5 & 646.0 & 19.2 & 2763 & 805 & 16.3 \\
half02B & 0.0120 & 4.7 & 679.3 & 17.8 & 2560 & 669 & 17.0 \\
half03B & 0.0053 & 3.2 & 465.5 & 17.9 & 2578 & 742 & 16.2 \\
half04D & 0.0053 & 4.1 & 594.0 & 18.0 & 2599 & 1079 & 16.8 \\
\hline Average & 0.0107 & 4.5 & 647.4 & 19.9 & 2859 & 861 & 15.9 \\
Std Dev & 0.0049 & 0.94 & 135.9 & 2.36 & 340 & 144 & 1.19 \\
COV & $46.0 \%$ & $21.0 \%$ & $21.0 \%$ & $11.9 \%$ & $11.9 \%$ & $16.7 \%$ & $7.5 \%$
\end{tabular}

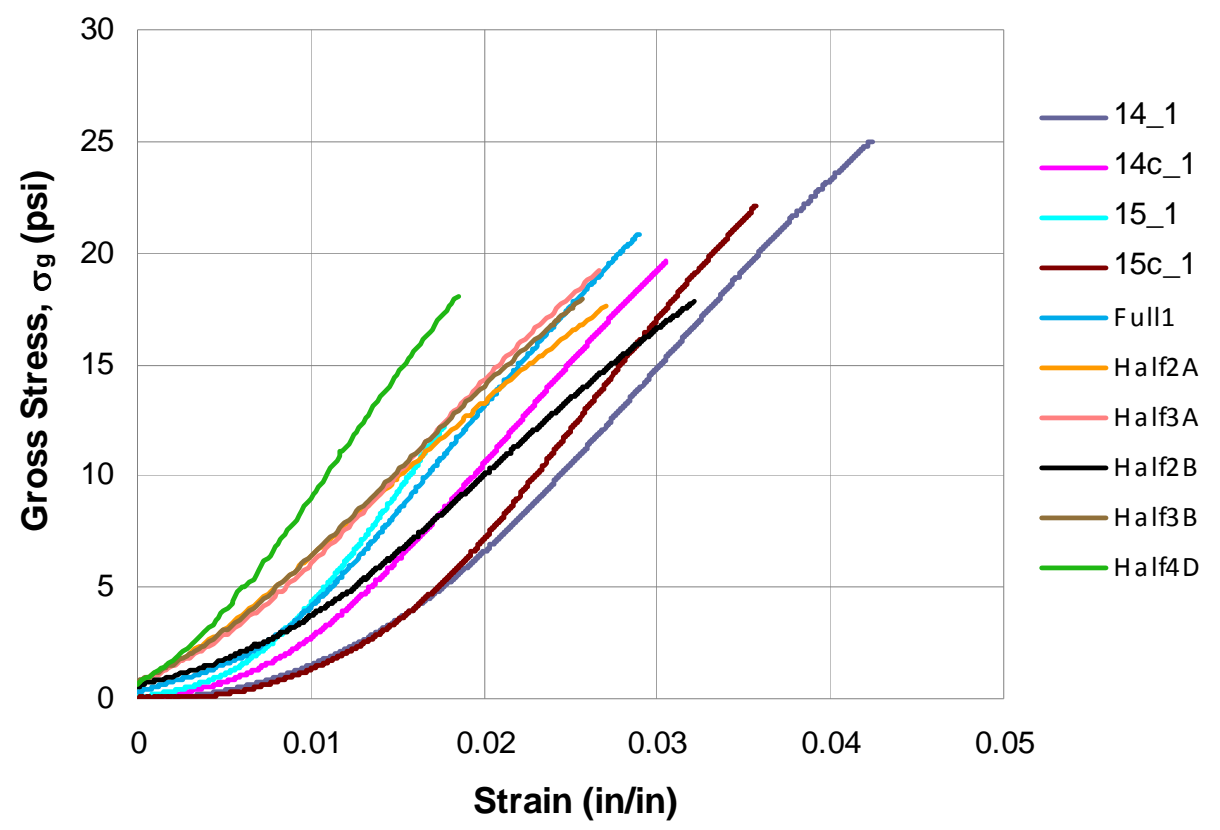

Figure 3-15: Summary of Preliminary Material Testing Results 
The results of the preliminary material testing have shown that the straw blocks hold up adequately to cyclic loading within their linear elastic range. Beyond this range of approximately 4.5 to 19.9 psi (647 to 2859 plf) the elastic moduli of the blocks drop significantly. This is true for both the low end within the initial toe and high end in the strain-softening region. Density appears to have a negative effect on block strength but positive in terms of reducing the settlement toe. Whether the loads on these straw blocks in a design-level seismic event will be outside this range will be investigated later in this report.

\subsubsection{Relaxation Testing over Time}

The decision to prestress the straw blocks for the shear wall testing and future construction, which will be discussed in detail later, came along with constructability issues. One of these constructability issues was that of relaxation of the blocks over time. When a block was loaded to a certain point (i.e. prestressed) and then that displacement held over time, the material will relax and dissipate this energy over time. How much load is lost and how long it takes for this to happen are both important factors in construction.

The relaxation testing was conducted on specimen Half04C. First the specimen was loaded to an assumed beginning of the linear elastic region of 1,000 psf (6.9 psi) and cycled five times. This was done both to simulate a block that was part of a structure and had undergone minor loading and unloading as well as to remove the initial settlement toe from the block specimen. After these five cycles, the block was loaded to an assumed proportional limit of 2,500 psf (17.4 psi) and held at this displacement (about 0.21 inches). This constant displacement was held for 48 hours straight. After 48 hours, the 
load had dropped significantly and the specimen was loaded again to its proportional limit. Figure 3-16 shows the results of this relaxation test.

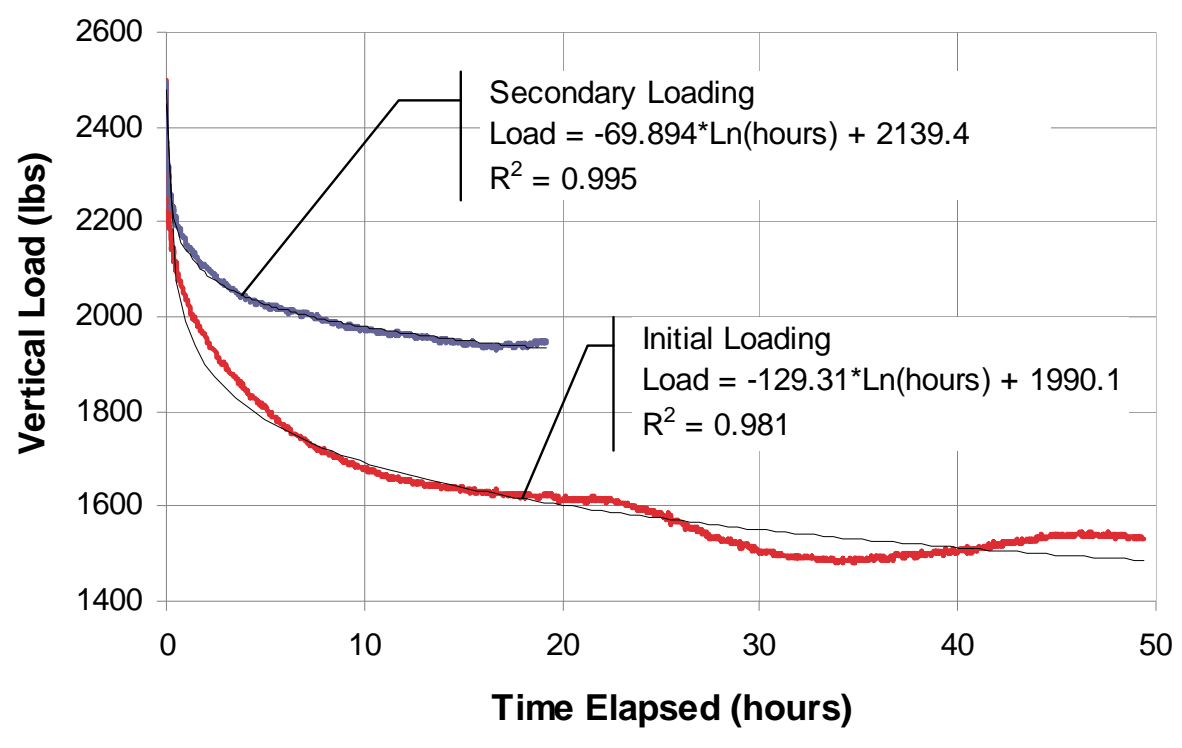

Figure 3-16: Relaxation Testing Results

While the secondary loading occurred immediately following the 48 hours of initial loading, the curves were placed on the same axis in order to compare the rate of load lost over time. The equations indicated next to each curve are logarithmic regressions from Microsoft Excel. The estimated load from these equations would be in pounds and time in hours. To help illustrate the difference in relaxation behavior Table 3-11 shows different estimated loads at projected times based on these results.

Table 3-11: Estimated Relaxation Losses

\begin{tabular}{ccc} 
Elapsed Time & $\begin{array}{c}\text { Initial Loading } \\
\text { (lbs) }\end{array}$ & $\begin{array}{c}\text { Secondary Loading } \\
\text { (lbs) }\end{array}$ \\
\hline \hline Initial & 2500 & 2500 \\
1 day & 1579 & 1917 \\
1 week & 1328 & 1781 \\
1 month & 1137 & 1678 \\
1 year & 816 & 1505 \\
10 years & 518 & 1344
\end{tabular}


While these values are only projections based on recorded data, they indicate how important a primary loading would be in the prestressed usage of these blocks. Much like what was found in the cyclic loading tests of the blocks, even though the block may yield on a material level when loaded to its proportional limit, there will be an increase in stiffness as many of the voids have closed.

Another important issue to note is the fluctuation seen after 24 hours in the initial loading curve in Figure 3-16. Figure 3-17 below plots this same loading data over time, with temperature and humidity measured outside the laboratory during the testing included for comparison.

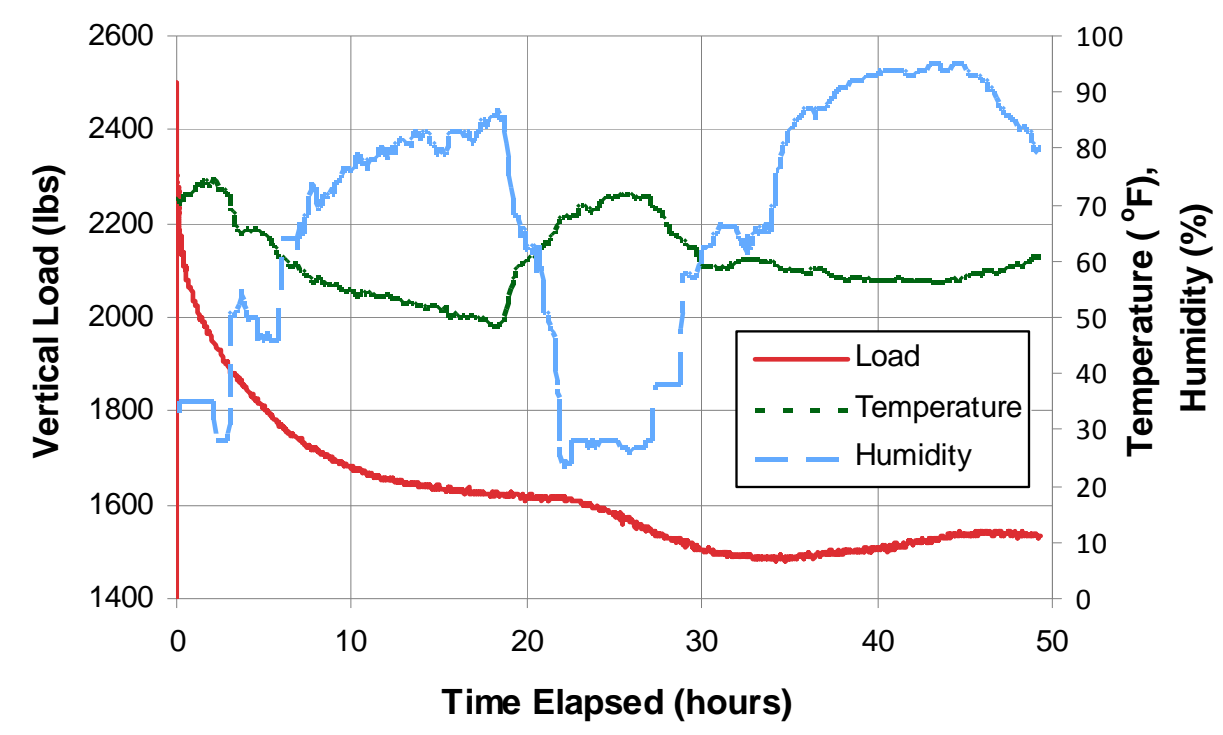

\section{Figure 3-17: Effects of Atmospheric Changes on Block Relaxation}

Although the laboratory was more of a controlled environment than the weather station on Cal Poly's campus at which the data was collected, the effects of atmospheric changes are still evident. The initial loading began around midday and after 20 hours was approaching a load of approximately 1,600 pounds. Yet at 24 hours there was a drop of approximately 200 pounds in the load on the block. This drop was followed by a 
morning increase in temperature $\left(50^{\circ}\right.$ to $\left.70^{\circ} \mathrm{F}\right)$ and an associated drastic fall in relative humidity (85\% to $25 \%)$.

This behavior indicates that when these straw blocks are in warmer, drier environments they lose strength. It can be seen that a slight increase in strength begins overnight around 40 hours elapsed as temperature has been lower and humidity is rising again. A possible explanation of this behavior could be that the straw fibers become more brittle when dried out or heated up and this causes a drop in stiffness and strength. While this drop is not drastic enough to be of concern, it should be noted in moving forward with construction waterproofing and material handling.

\subsubsection{Block Material Properties Post-Shear Wall Testing}

Following the shear wall testing portion of this investigation, which will be discussed in the subsequent chapters, blocks from each wall were removed from the wall assemblies and tested. This testing of used blocks was important to investigate not only the behavior following the prestressing loads, but also the effects of certain construction modifications on the blocks.

Two or three blocks from each shear wall were tested immediately following each experiment. The entire used block testing program is described below in Table 3-12. All blocks were tested at the same load rate as before ( 0.12 inches per minute) unless noted otherwise. Also, the first three blocks (wall1_D3, wall1_E2, and wall2_A4) were loaded out to higher strain levels (50\%) to investigate behavior in this region while the remainder was only loaded one cycle out to $10 \%$. 
Table 3-12: Used Block Testing Program

\begin{tabular}{cc} 
Specimen & Description \\
\hline \hline wall1_D3 & Full Block 12" Tall ( ${ }^{\star} 0.24$ in/min) \\
wall1_E2 & Full Block 12" Tall ( ${ }^{*} 0.24$ in/min) \\
wall2_A4 & Full Block 9.375" Tall (Bottom Removed) \\
wall2_C3 & Full Block 12" Tall with Screw Damage \\
wall2_H5 & Half Block 9.375" Tall (Top Removed) \\
wall3_A4 & Full Block 9.375" Tall (Bottom Removed) \\
wall3_H5 & Half Block 9.375" Tall (Top Removed) \\
wall4_D2 & Full Block 12" Tall with Inspection Hole \\
wall4_H5 & Half Block 9.375" Tall (Top Removed)
\end{tabular}

Although the walls were all prestressed in a manner described in the following report, every specimen still exhibited the initial toe region that was seen in prior materials testing. The results from all of the used block tests are found below in Table 3-13.

Table 3-13: Used Block Test Results

\begin{tabular}{cccc} 
specimen & $\begin{array}{c}\sigma_{\mathrm{g}, \mathrm{prop}} \\
(\mathrm{psi})\end{array}$ & $\begin{array}{c}\sigma_{\mathrm{g}, \mathrm{prop}} \\
(\mathrm{psf})\end{array}$ & $\begin{array}{c}\mathrm{E}_{\mathrm{g}} \\
(\mathrm{psi})\end{array}$ \\
\hline \hline wall1_D3 & 22.7 & 3266 & 799 \\
wall1_E2 & 21.9 & 3155 & 833 \\
wall2_A4 & 27.5 & 3958 & 573 \\
wall2_C3 & 25.4 & 3663 & 955 \\
wall2_H5 & 22.9 & 3290 & 534 \\
wall3_A4 & 29.8 & 4291 & 431 \\
wall3_H5 & 17.8 & 2568 & 490 \\
wall4_D2 & 21.6 & 3116 & 563 \\
wall4_H5 & 16.1 & 2321 & 499 \\
\hline Average & 22.9 & 3292 & 631 \\
\hline Prior Avg. & 19.9 & 2859 & 861
\end{tabular}

It can be seen in the results above that the proportional limits are comparable, if not higher, than the unused blocks. Also, there was a significant reduction in stiffness (27\%) between the blocks used in the shear walls and the prior testing. This change is not due to variations in block manufacturing, but instead appears to be caused by 
modifications done on the blocks during the construction process. Table 3-14 outlines the different potential factors for the drastic elastic moduli reductions.

Table 3-14: Block Modification Effects on Strength and Stiffness

\begin{tabular}{cccc} 
Factor & $\begin{array}{c}\sigma_{\mathrm{g}, \text { prop }} \\
(\mathrm{psi})\end{array}$ & $\begin{array}{c}\sigma_{\mathrm{g}, \text { prop }} \\
(\mathrm{psf})\end{array}$ & $\begin{array}{c}\mathrm{E}_{\mathrm{g}} \\
(\mathrm{psi})\end{array}$ \\
\hline \hline 12" Tall & 20.7 & 2975 & 861 \\
9.375" Tall & 22.8 & 3286 & 505 \\
Full Width & 23.4 & 3365 & 800 \\
Half Width & 18.4 & 2651 & 686 \\
Top Removed & 18.9 & 2727 & 508 \\
Bottom Removed & 28.6 & 4124 & 502 \\
Inspection Hole & 21.6 & 3116 & 563
\end{tabular}

The table above includes the half and full block data from the preliminary material testing as well as all of the blocks from the shear wall testing program. It should be noted that there are overlaps within the modification factors; for example the $9 \frac{3}{8}$ inch tall blocks have either had the top or bottom $2 \frac{5}{8}$ inches removed or a full width block could be shorter than 12 inches.

The most basic finding of this investigation is that any alteration will significantly reduce the elastic modulus of the block. Removing height from a block appears to have the greatest negative effect, whether it is off the top or the bottom. Removing a $2 \frac{1}{2} \mathrm{inch}$ diameter plug for an inspection hole also reduced the stiffness of the tested blocks, although not as much.

The same does not hold true for the proportional limit strength of the blocks. Whereas removing the top or the bottom had the same negative effect on the stiffness, the yield strength was reduced when the top portion was removed and greatly increased when the bottom portion was removed. This appears to be a byproduct of the manufacturing process: the straw material at the bottom of the block appears to be 
weaker than at the top and yields first. This could be caused by the orientation of the straw within the molds or even the placement of the straw at the bottom of the block versus the top.

Removing half of the block width with a sawing process has an even greater effect on the yield strength. The inspection hole had a comparable yield strength to the untouched blocks so it can be assumed that this hole only affects stiffness and not strength.

\subsubsection{Comparison to Straw Bale Testing}

The strength of straw bale has been reported over various testing programs as ranging from 17 to 84 psi. These values are based on varying straw sources, baling processes, moisture content, and even testing procedures. The amount of information presented from each of these testing programs also varies, but from what is reported, straw bale does not exhibit the same three "regions" as the blocks defined in Figure 3-4. The initial toe settlement region is not as obvious and a clear linear elastic region does not appear to be present. The bales tested exhibited a strain-hardening behavior at higher strains which is different than what was seen in the block testing program in this investigation.

Yet, in Bou-Ali’s (1993) testing program, the bales were loaded out to strains as high as 58\%. This strain would translate to a deflection of 4'-8” of an 8'-0" tall wall. It is at this strain that the ultimate strengths of the straw bales were reported. More recent literature such as King (1998) cites the accepted strength of straw bales used in construction as 70 psi. Looking at Bou-Ali's data, straw bales would not reach this 
strength until 50-55\% strain which is unreasonable to anticipate in any type of construction.

Using a similar method of linear regression fitting to determine an elastic modulus and yield strength (although the specimens exhibited strain-hardening at the end of this linear elastic region), Bou-Ali’s specimens had Young’s Moduli of approximately 68 psi and proportional limits of 19 psi. While these values are very close to those Field et al. (2005) reported; they are significantly lower than the strengths being reported and accepted as material strengths.

For comparison purposes, two blocks in this testing program were loaded out to extremely high strains to see if the strain-hardening behavior would occur. The results from these tests are found in Figures 3-18 and 3-19.

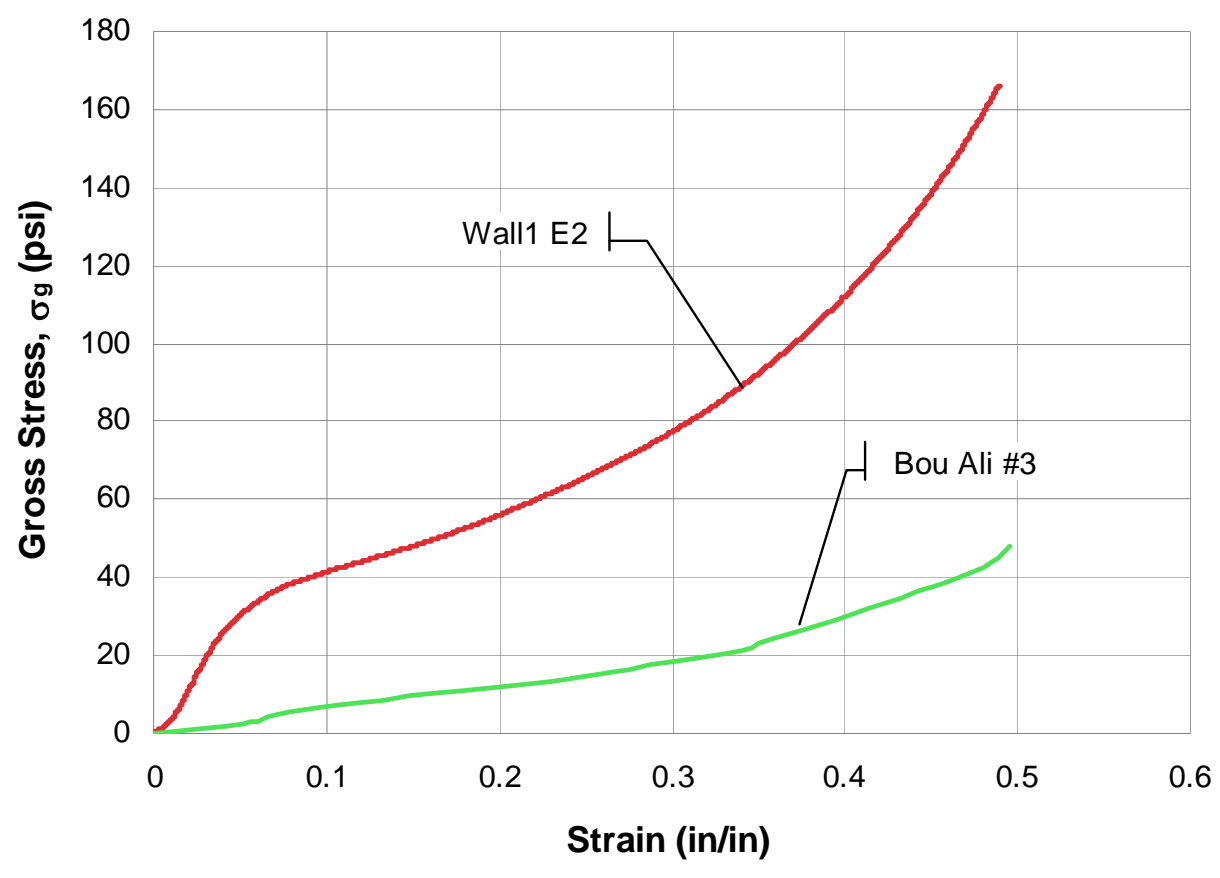

Figure 3-18: Comparison of Straw Blocks to Bales at High Strains 


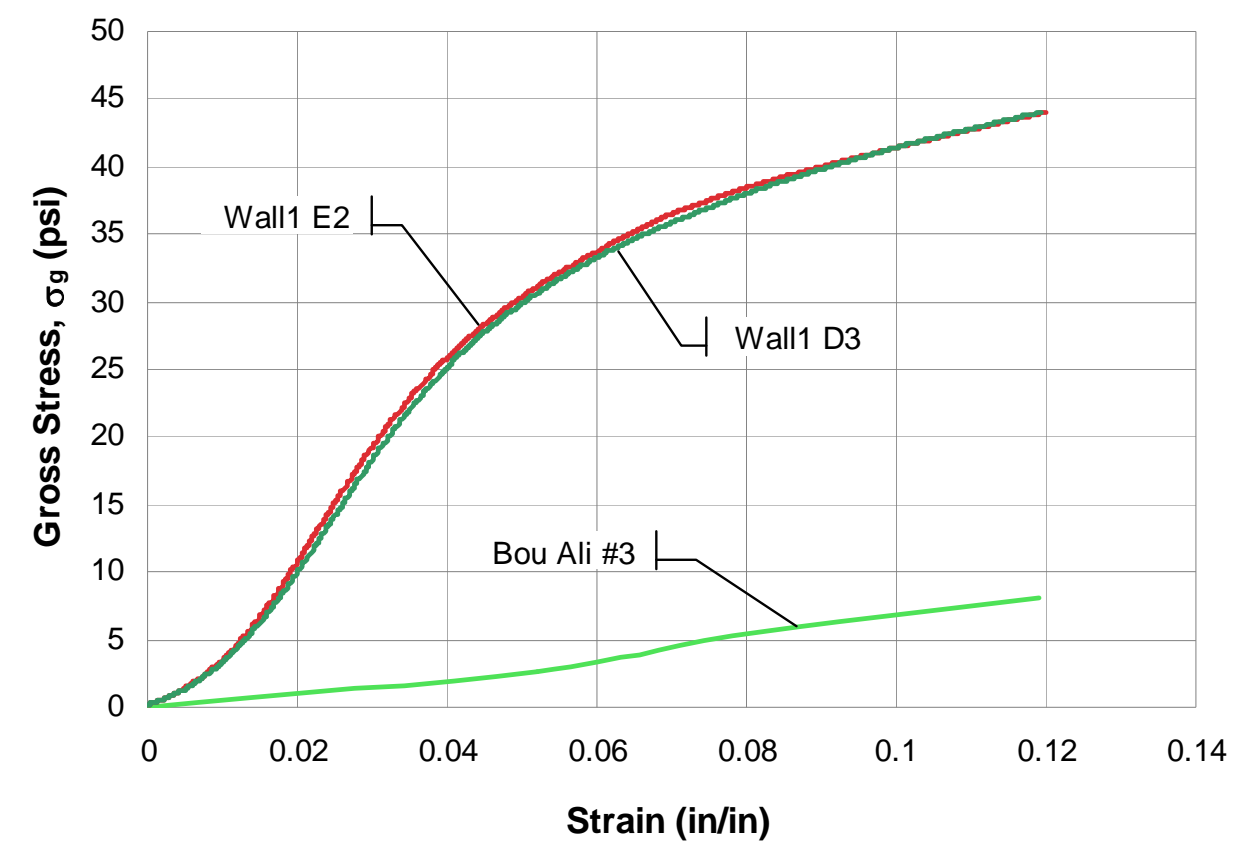

Figure 3-19: Comparison of Straw Blocks to Bales up to $12 \%$ Strain

Figure 3-18 shows that the straw blocks exhibit extreme strain hardening behavior past a strain of $20 \%$. Yet, it is not correct to call the maximum load of 167 psi it reached during testing an "ultimate load” because the block will never fail; instead the block would continue to harden and compress until it reaches $100 \%$ strain. Doing this could potentially damage the testing equipment and does not provide useful structural information. Figure 3-19 shows more appropriate strain values and how the blocks compare to straw bales. This figure further illustrates the relative increase in stiffness between the two materials that was discussed earlier.

\subsection{Prestressing Materials Investigation}

The materials investigation of the blocks has provided insight into how they would behave under typical residential loads. For example, a single story residential structure designed per ASCE 7-05 (American Society of Civil Engineers, 2006) loading 
could see loads throughout its life ranging from 200 plf to 1,400 plf (assuming 10'-0” of roof tributary width bearing on the wall). The linear elastic range of the blocks was measured to be from approximately 650 plf to 2860 plf. This linear elastic range of the blocks is very important to stay within during the life of the structure because on each end outside of this range, the blocks exhibit much softer behavior.

It was decided that because of this material behavior that the wall assemblies would be prestressed prior to construction and testing. The prestressing behavior and details will be discussed in much greater detail later in the report, but on a basic level, a prestressing material will pass through the cylindrical voids that are leftover from the manufacturing molds and attach to the foundation.

The prestressing was accomplished either using steel threaded bars or braided wire ropes. Unlike efforts to reinforce straw bale with rebar pins resisting shear forces, the bars or rope will only see tensile forces. The predicted magnitudes of these tensile forces are dependent on the wall detailing will be discussed in the next chapter, but both materials used in construction were tested to investigate the strengths of the actual materials being used.

\subsubsection{Tensile Testing Setup}

As mentioned before, the tensile testing was performed on a 120 kip capacity MTS test frame in the Cal Poly Advanced Materials Laboratory. The thread bar samples were attached to the test frame in the manner shown in Figure 3-20. The specimens were nominally 12 inches in length with about one inch of each end threaded into attachments on the test machine. An extensometer, shown in Figure 3-20, with an 8” gage length was attached to the sample to measure strain. 

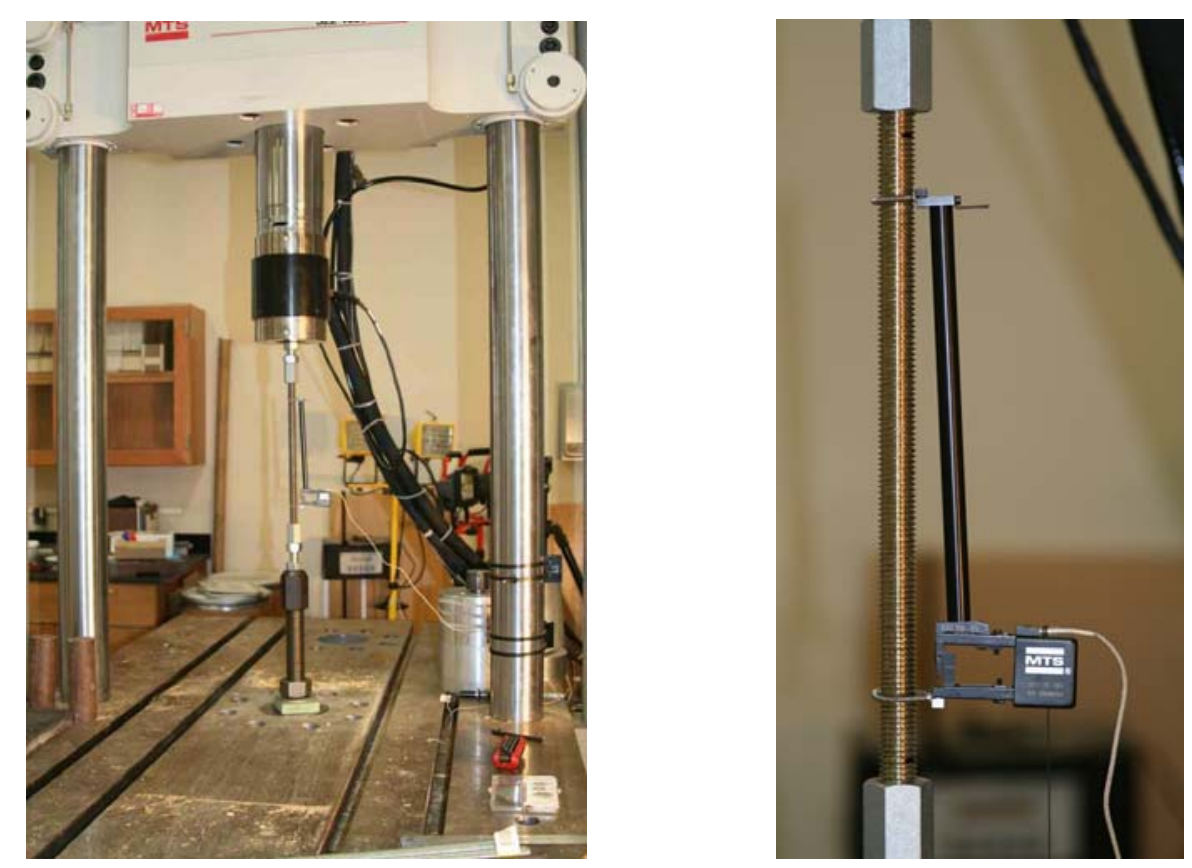

Figure 3-20: Sample Prestressing Tensile Testing Configuration

\subsubsection{Threaded Rod Testing}

The thread bars used in wall construction would have to be at least 8'-0” long and threaded rod of this length is typically used for industrial purposes such as hanging HVAC and lighting equipment form the ceiling. Because of the small loads associated with this application, the grades of steel used in manufacturing were not always indicated by the supplier. To investigate the strengths available for purchase, a variety of samples were obtained from different manufacturers for two bar diameters ( $1 / 2$ and $5 / 8$ inch). Table 3-15 lists all of the tested threaded bar samples. For the testing of full-scale shear walls, the thread bar used in construction was $1 \frac{1}{2}$ ” A-1 for Wall 1 and $1 / 2$ ” D-1 was used in Walls 2 and 4. 
Table 3-15: Tested Thread Bar Samples

\begin{tabular}{ccccc} 
Specimen & Source & Description & $\begin{array}{c}\text { Date } \\
\text { Obtained }\end{array}$ & Comments \\
\hline \hline 1/2" A-1 & Grainger & Gold Galv & $2 / 20 / 2009$ & Shipment \#1, used in Wall 1 \\
1/2" B-1 & Pacific H\&G & Hot dipped Galv & 2006 & \\
1/2" C-1 & Home Depot & Hot dipped Galv & 2006 & \\
1/2" D-1 & Grainger & Gold Galv & $2 / 26 / 2009$ & Shipment \#2, used in Wall 2 \& 4 \\
5/8" A-1 & McCarthy & Black & 2006 & Insufficient data collected \\
5/8" A-2 & McCarthy & Black & 2006 & Same rod as 5/8" A-1 \\
5/8" B-1 & Pacific H\&G & Hot dipped Galv & $2 / 26 / 2009$ & \\
5/8" C-1 & Grainger & Gold Galv & $2 / 26 / 2009$ & Came in shipment \#2
\end{tabular}

Each sample was fitted with a strain gauge measuring device to accurately capture the yield points, but these gauges were removed at higher strains to avoid damage. The samples were all 12 inch lengths and were loaded at a rate of 0.05 inches per minute to the point of failure.

ASTM F1554 outlines specifications for various grades of threaded bar. These required strengths are listed in Table 3-16.

Table 3-16: ASTM F1554 Threaded Bar Grades

\begin{tabular}{cccc} 
& Grade 36 & Grade 55 & Grade 105 \\
\hline \hline Yield Strength, Min. (ksi) & 36 & 55 & 105 \\
Tensile Strength, Min. (ksi) & 58 & 75 & 125 \\
Tensile Strength, Max. (ksi) & 80 & 95 & 155 \\
Elongation, Min. (\%) & 20 & 18 & 12
\end{tabular}

Figures 3-21 and 3-22 and Tables 3-17 and 3-18 show the results from the testing for the $1 / 2$ inch diameter and $\frac{5}{8}$ inch diameter specimens, respectively. The dashed horizontal lines on the plots represent the three ASTM F1554 requirement stresses for Grade 55 steel. The effective tensile stress area $\left(0.1419 \mathrm{in}^{2}\right.$ for $1 / 2$ inch diameter and 0.226 in $^{2}$ for $\frac{5}{8}$ inch) of the bars was determined per ASTM F1554-07. 


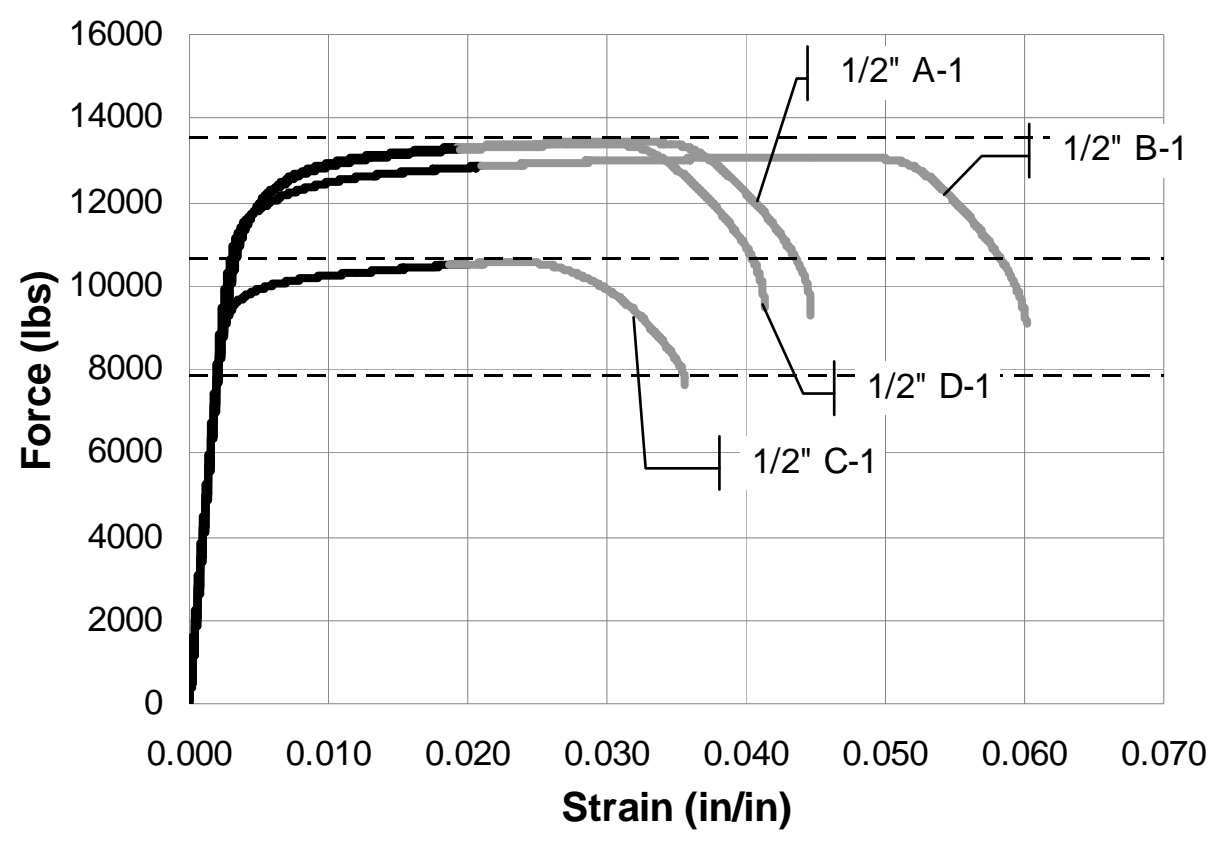

Figure 3-21: 1/2" Diameter Thread Bar Results

Table 3-17: 1/2" Diameter Thread Bar Results

\begin{tabular}{ccccc} 
Specimen & $\begin{array}{c}\text { Max Load } \\
(\mathrm{lbs})\end{array}$ & $\begin{array}{r}\text { Tensile Strength } \\
(\mathrm{ksi})\end{array}$ & $\begin{array}{c}\text { Yield Force } \\
(\mathrm{lbs})\end{array}$ & $\begin{array}{c}\text { Yield Strength } \\
(\mathrm{ksi})\end{array}$ \\
\hline \hline 1/2" A-1 & 13446 & 94.8 & 12000 & 84.6 \\
1/2" B-1 & 13059 & 92.0 & 11800 & 83.2 \\
1/2" C-1 & 10559 & 74.4 & 9850 & 69.4 \\
1/2" D-1 & 13382 & 94.3 & 12000 & 84.6
\end{tabular}




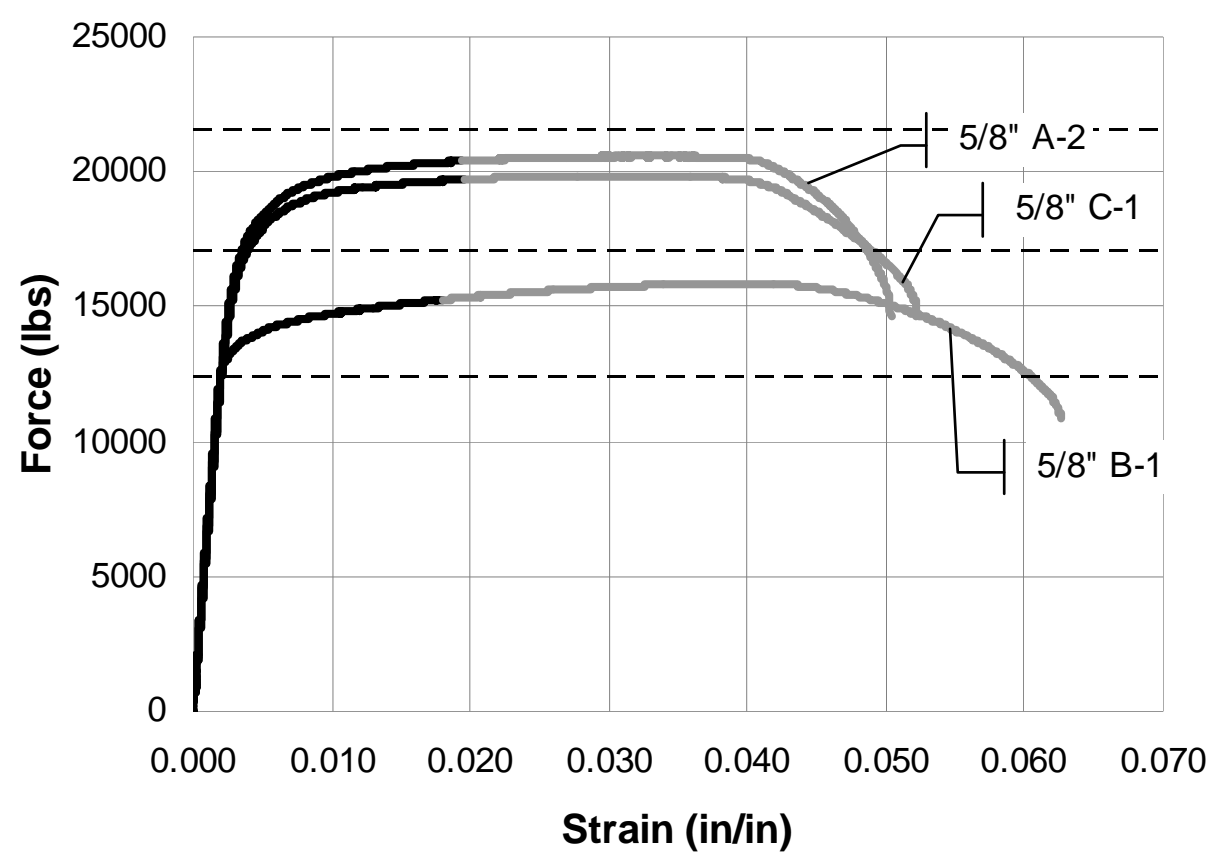

Figure 3-22: 5/8" Diameter Thread Bar Results

Table 3-18: 5/8" Diameter Thread Bar Results

\begin{tabular}{ccccc} 
Specimen & $\begin{array}{c}\text { Max Load } \\
(\mathrm{lbs})\end{array}$ & $\begin{array}{c}\text { Tensile Strength } \\
(\mathrm{ksi})\end{array}$ & $\begin{array}{c}\text { Yield Force } \\
(\mathrm{lbs})\end{array}$ & $\begin{array}{c}\text { Yield Strength } \\
(\mathrm{ksi})\end{array}$ \\
\hline \hline 5/8" A-1 & 20190 & 89.3 & & \\
5/8" A-2 & 20584 & 91.1 & 18356 & 81.2 \\
5/8" B-1 & 15875 & 70.2 & 13891 & 61.5 \\
5/8" C-1 & 19867 & 87.9 & 17865 & 79.0
\end{tabular}

The lines plotted in the figures above are black to represent the strain gauge readings and then grey to plot the data after the strain gauge was removed and the displacement was used to calculate strain. It can be seen that the strengths of thread bar from various sources can vary even within the same steel grade. Two samples, 1/2" C-1 and $5 / 8$ " B-1 (both from local hardware stores), do not meet the ASTM requirements for Grade 55 steel, and instead would be classified as Grade 36 due to their maximum tensile strengths. The thread bar from Grainger, used in wall testing (1/2” A-1 and 1/2” D-1) exhibited practically identical behavior. 


\subsubsection{Wire Rope Testing}

Another prestressing option that was investigated was the use of wire rope. Loops of $1 / 4$ inch diameter wire rope would be used in lieu of $1 / 2$ inch thread bar to see how the prestressed wall behavior was affected. The construction details of using the wire rope will be discussed later, but for materials testing 7x19 strand core wire rope was looped through two eyebolts and tested in the same manner as the thread bar. The wire ropes had a safe working limit of 3,100 lbs listed on its packaging. For wire rope or cable, a safe working load typically has a factor of safety of 3-5 included under the breaking strength.

A 27 inch length (eyebolt to eyebolt) of wire rope was tested to failure at a rate of 0.29 inches per minute. The wire rope samples failed in a series of strands snapping as shown in Figure 3-23. The results from the testing are found below in Figure 3-24. The strain was calculated by using the recorded test machine head deflection divided by the specimen’s initial length.

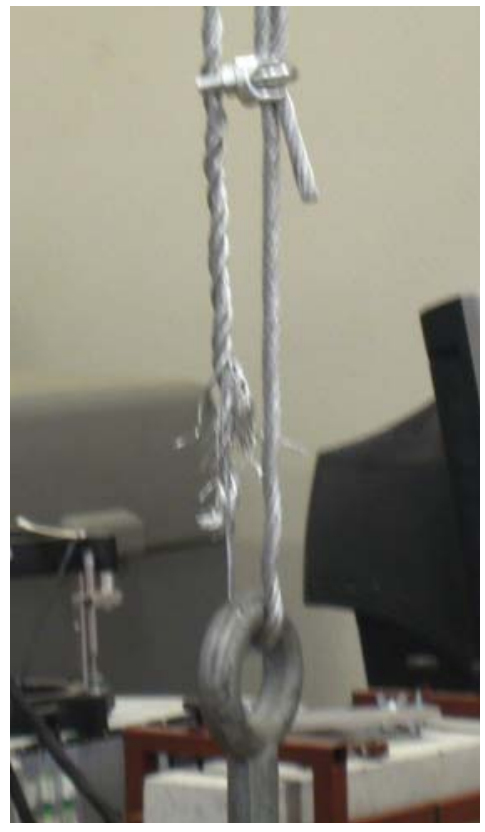

Figure 3-23: Wire Rope Failure 


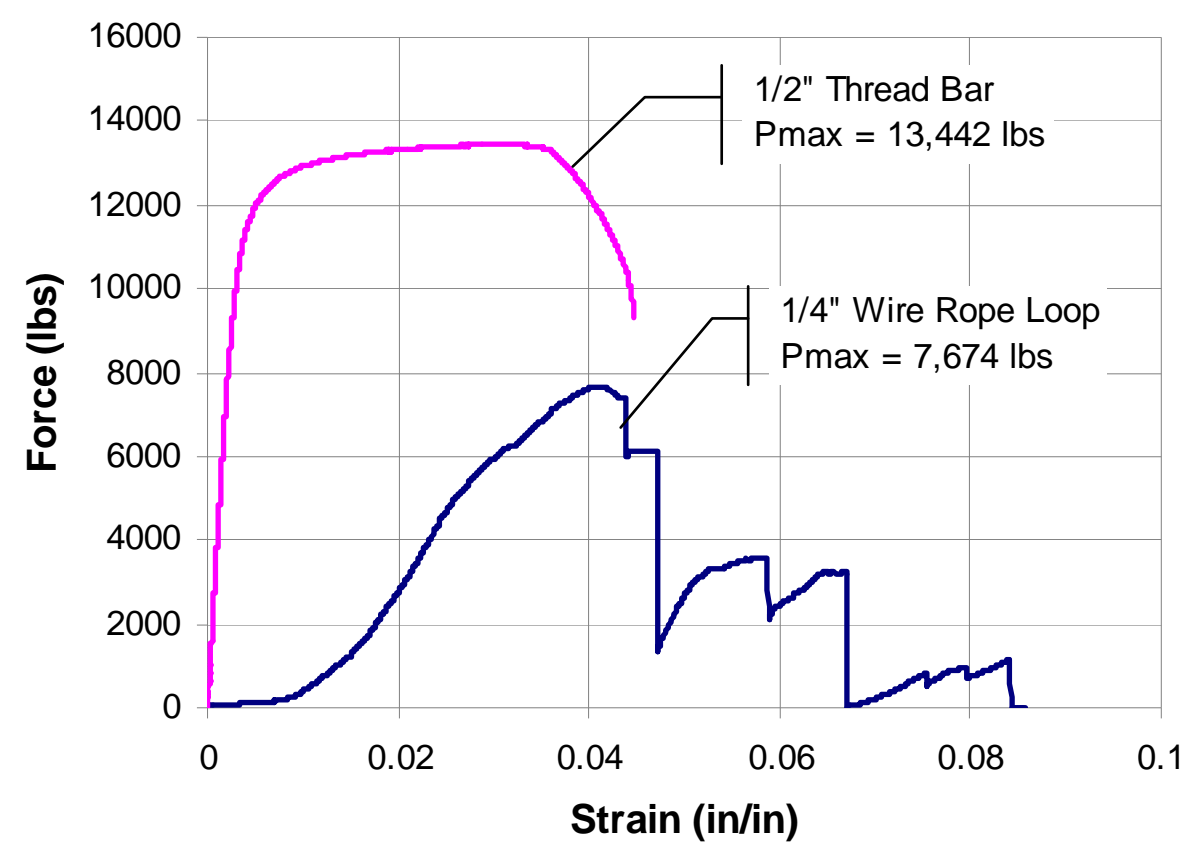

Figure 3-24: Comparison of Thread Bar to Wire Rope Prestressing

\subsubsection{Prestressing Material Discussion}

The results of the wire rope test showed the difference in material behavior. For example, an initial "toe" can be seen in the wire rope because although the loop was tightened prior to testing, some slack was still present at the lower strains. Also, since the rope is braided, the fibers will pull close to one another until they are taut and the rope enters a linear elastic region. The failure of the specimen occurred where the rope was bent sharply around the eye bolt and because of this concentration of force the rope failed at a lower force than expected.

The failure was not as ductile as the thread rod because of what occurred on a material level. The first strand broke around 0.033 in/in where a slight jog can be seen in the stress-strain curve and then the subsequent steps are more and more strands fraying 
and the net area becoming smaller. This failure is not desirable in a seismic application because of the sudden drop in force of nearly 6,000 lbs.

A larger diameter wire rope would have been tested, but constructability and connection issues come with diameters greater than a quarter inch. For example, the simple u-bolt clamps can no longer be used to splice two rope sections. Because of the difficulties in using larger diameters and the specialized tools required, a readily available, easy to use size was chosen.

Even though the materials are similarly priced, because of the initial settlement issues and lower strengths associated with wire rope, threaded bar is the superior prestressing material. One half inch diameter threaded bar should be able to handle the magnitude of forces expected in straw block construction. The associated construction detailing and issues that surfaced during wall construction will be discussed in the following chapter.

\subsubsection{Potential for Bamboo Prestressing}

In the interest of keeping the construction of these straw block walls sustainable and environmentally responsible, a potential alternative to the steel rod or wire could be bamboo. Bamboo is a rapidly renewable, low-cost material that has been used in construction in countries such as China, India and Japan for centuries. Although testing and building with bamboo prestressing was outside of the scope of this report, an investigation into this prestressing method is recommended for future research.

Although the steel thread bar performed as needed, steel as a material is known to contain a very high embodied energy for a building material. Embodied energy is the total amount of energy consumed in the extraction, manufacture, transport, construction 
and assembly on site of building materials. Table 3-19 outlines the embodied energy for the building materials used in this study, by weight, as reported by the Centre for Building Performance Research (2003) and Reiner et al. (2007).

Table 3-19: Embodied Energy of Materials Used

\begin{tabular}{cccc} 
Material & MJ/kg & kWh/lb & kWh/8 ft wall \\
\hline \hline Concrete & 1.3 & 0.16 & $491.31^{*}$ \\
PT Sill Plate & 2.5 & 0.31 & 15.12 \\
LSL Top Plate & 11.0 & 1.39 & 70.67 \\
OSB & 8.0 & 1.01 & 84.66 \\
Straw Bale** & 0.2 & 0.03 & 30.23 \\
Steel (recycled) & 10.1 & 1.27 & 20.82 \\
Steel (virgin) & 32.0 & 4.03 & 65.95 \\
Bamboo (local) & 0.02 & 0.002 & 0.03 \\
Bamboo (China) & 5.4 & 0.69 & 11.23 \\
*Same foundation was used for all four wall tests \\
**Straw Block Data not available
\end{tabular}

The table above shows the fact that although steel does contain high embodied energy, so little is used in the construction of a wall that not much is gained by changing to bamboo. Also, the source of the building material has a very large effect on the amount of energy associated with it. Bamboo sourced locally has practically zero embodied energy, while bamboo from the Hunan Province of China (the origin of the majority of bamboo used in construction) has significantly larger embodied energy due to the overseas transport to the United States. Because of this, steel originating from China can have two to three times more embodied energy due to transportation.

So although the concrete, and engineered wood products contribute much more in terms of embodied energy to this structural system, the use of bamboo might still be desirable for novelty purposes or if the straw blocks were to be used in an area such as Asia where bamboo is more prevalent. In these instances, it would be useful to know that 
bamboo would perform comparably to the steel materials described in the following chapter. Table 3-20 summarizes the research done on bamboo and compares the mechanical properties to that of the steel thread rod.

Table 3-20: Bamboo Material Properties

\begin{tabular}{ccc} 
Study & $\begin{array}{c}\sigma_{\mathrm{u}} \\
(\mathrm{ksi})\end{array}$ & $\begin{array}{c}\mathrm{E} \\
(\mathrm{psi})\end{array}$ \\
\hline \hline Brink et al. (1966) & 18.0 & $2.50 \mathrm{E}+06$ \\
Nirman (2009) & 15.3 & $1.67 \mathrm{E}+06$ \\
Carrasco et al. (2002) & 14.2 & $2.27 \mathrm{E}+06$ \\
Jain et al. (1992) & 29.1 & -- \\
Amada and Untao (2001) & 26.8 & $2.03 \mathrm{E}+06$ \\
\hline Bamboo Average & 20.7 & $2.12 \mathrm{E}+06$ \\
1/2" Dia Rod & 88.9 & $3.44 \mathrm{E}+06$
\end{tabular}

While sometimes it has been reported that bamboo is stronger in steel, in this application, this is definitely not the case. Even though bamboo is impressively strong for a naturally growing material, its strength is four times lower than steel. This translates to needing bamboo stalks, or culms, with twice the diameter as the steel rod used in this study. While one inch diameter bamboo is not unheard of or unwieldy, if this material is substituted, a larger size should be used.

Another issue with using bamboo would be creating viable connections to handle the expected tensile loads. In some cases, drilling and epoxying bamboo into concrete in a similar manner to reinforcing steel has been used in construction. This method, along with other connection options to the concrete foundation should be investigated and tested before put to use in construction. The connection at the top plate where the prestressing is performed is another issue that would have to be figured out. With the threaded bar, this was accomplished simply by tightening the nut; a method to prestress 
bamboo stalks would need to be developed in order to achieve similar structural system behavior. Eventually a wall made of compressed straw blocks, prestressed with bamboo, with sheathing, top and sill plates made of an engineered straw or bamboo product might be possible. This would reduce the embodied energy of the system and allow for more rapidly renewable materials to be utilized. For the time being, steel thread bars seem to be the most practical and relatively low embodied energy option.

\subsection{Plywood Fastening Investigation}

Although the aim of this study is to investigate the feasibility of the straw blocks as a load-bearing wall, details such as the aforementioned prestressing will combine with the blocks to create a superior structural system. One of these details is the use of plywood panels attached to the straw block wall to provide increased strength.

The relatively low density of the straw blocks (one half that of Douglas Fir Larch lumber) allows for easy fastening to the blocks. Yet, this low density also means the fasteners need to be different than those used in conventional wood construction. For example, the eight-penny nails typically used to attach sheathing to wood studs could not be used to attach to the straw blocks. Nails can be pushed completely in by hand and do not need to be hammered into the blocks. This ease translates to practically null withdrawal resistance.

It was decided that screws would be the appropriate fastening method to attach the sheathing to the straw blocks and effectively transfer shear loads. Since so many screws would be needed to attach a wall panel to the straw blocks, low-priced, readily available screws were chosen although fasteners such as deck screws could have provided greater strength and pullout resistance. Standard gold screws were chosen in 
four different lengths $\left(1 \frac{5}{8}, 2,21 / 2\right.$, and 3 inch) for the investigation into appropriate fasteners.

\subsubsection{Testing Setup}

For the testing program, a mockup double shear specimen was built using one half straw blocks in between two $1 / 2$ inch thick plywood panels. The panels were attached on each side to the block with four screws spaced 4 inches apart. A 2x member was placed as a strut in between the panels to not allow peeling away and to force pure shear transfer. The test setup configuration is shown in Figure 3-25.

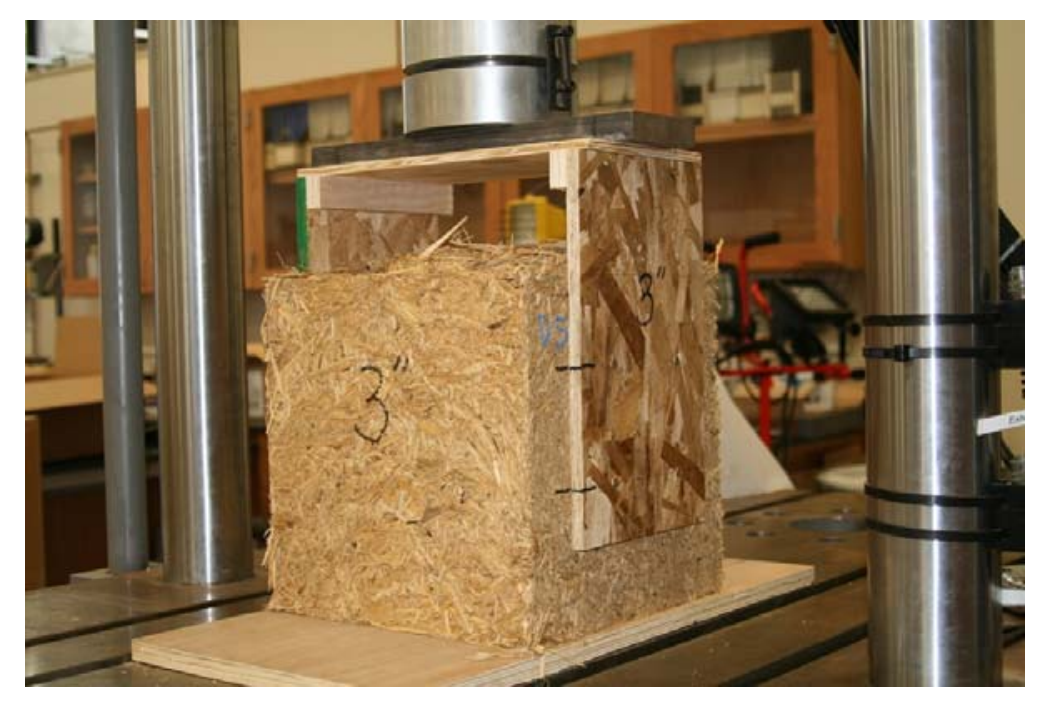

Figure 3-25: Screw Testing Setup

Each specimen was loaded at 0.25 inches per minute to failure on the same MTS test frame as used in all materials testing to this point. Failure of the specimens was determined by having reached a peak load and then a subsequent loss of one half of that load with increased displacement. For comparison purposes, an additional control sample was constructed using two 2x Douglas Fir members in between two 1/2 inch plywood panels attached with eight 8d nails (four each side) shown in Figure 3-26. 


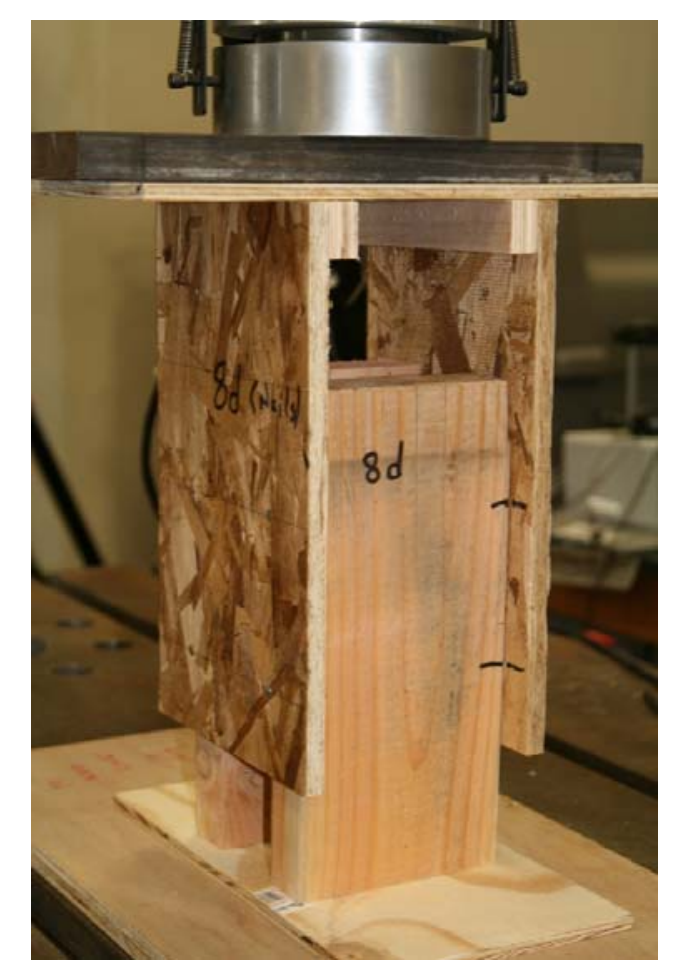

Figure 3-26: Nail Comparison Testing Setup

\subsubsection{Screw Testing Results}

The results from the shear testing of the screws and nails are found below in

Figure 3-27 and Table 3-21.

Table 3-21: Screw Shear Testing Results

\begin{tabular}{cccc} 
Type & $\begin{array}{c}\text { Yield Shear } \\
\text { (lbs/fastener) }\end{array}$ & $\begin{array}{c}\text { Strain @ Yield } \\
\text { (in/in) }\end{array}$ & $\begin{array}{c}\text { Max Shear } \\
\text { (lbs/fastener) }\end{array}$ \\
\hline \hline 1 5/8" Screw & 45 & 0.08 & 85.3 \\
2" Screw & 53 & 0.098 & 115.4 \\
2 1/2" Screw & 57 & 0.106 & 177.3 \\
3" Screw & 65 & 0.119 & 201.3 \\
8d Nail & 186 & 0.052 & 410.3
\end{tabular}




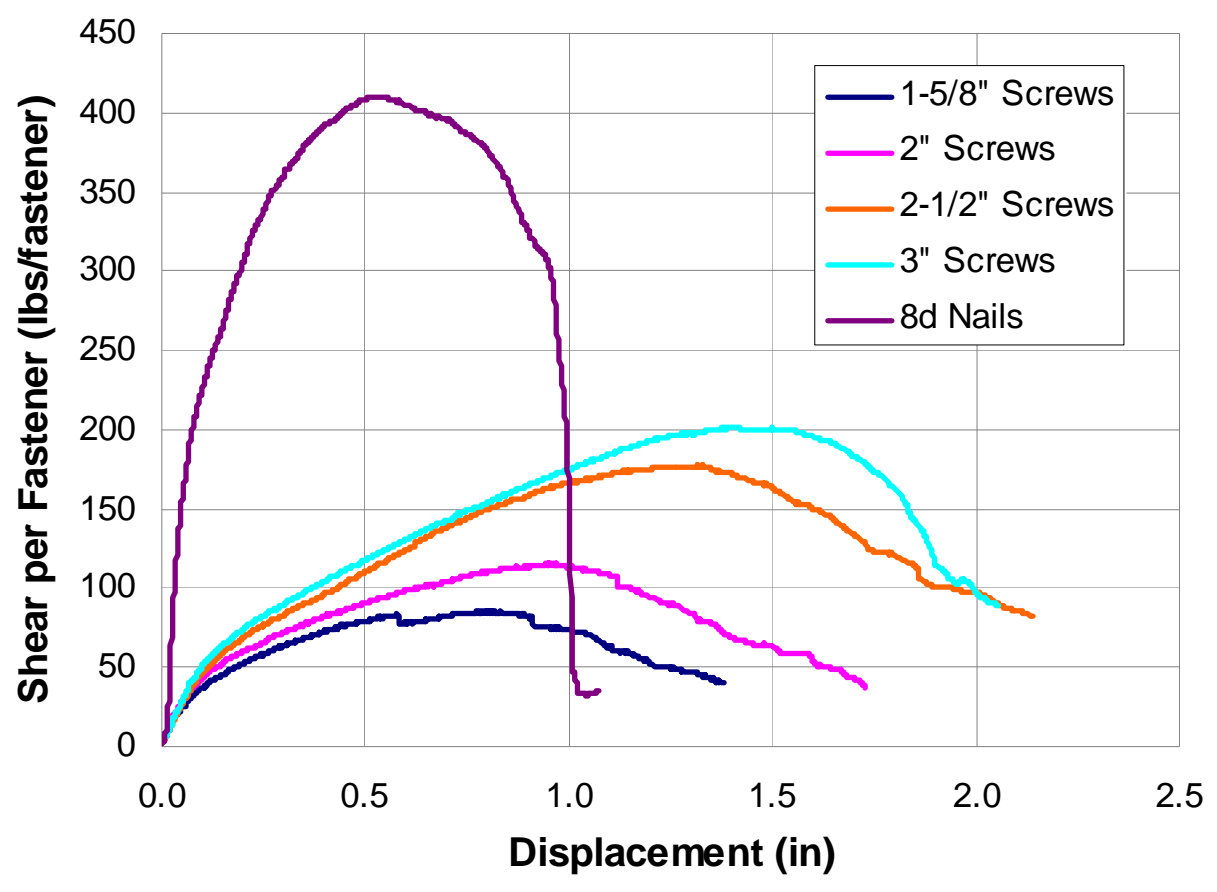

Figure 3-27: Screw and Nail Shear Testing Results

The figure above illustrates first how soft the straw blocks are versus lumber in the initial slopes of the lines. This is because the failure mechanism of the tested screws was slipping within the straw material whereas the nails themselves yielded. It was expected that the nails would perform better, but the purpose of this investigation was to determine the order of magnitude of how much weaker the screws and straw blocks would perform (3 to 4 times weaker). Also, the effects of the screw lengths are clearly seen in the results above.

The yield points of the nails and screws were determined using a bilinear approximation as shown in Figure 3-28. 


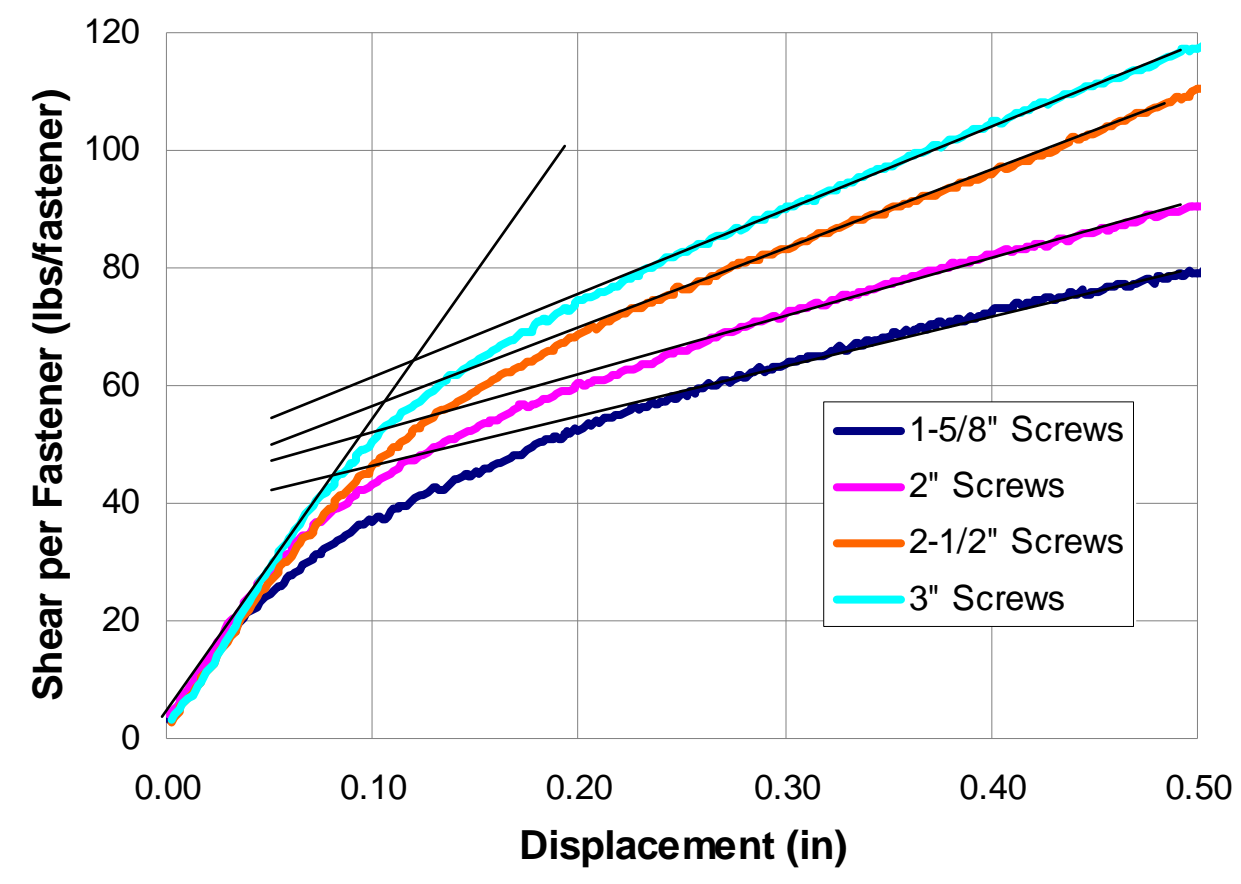

Figure 3-28: Bilinear Approximation of Screw Yield Points

A similar method was used to obtain the yield shear strengths of the nails, which was about twice the design values given in the NDS (186 versus $88 \mathrm{lbs} /$ fastener) due to conservatism. The yield point of the screws in the straw blocks could be seen physically when the screws started to slip (Figure 3-29). This slip was directly dependent on the length of the screw because all four lengths followed the same initial slope in Figure 326. 


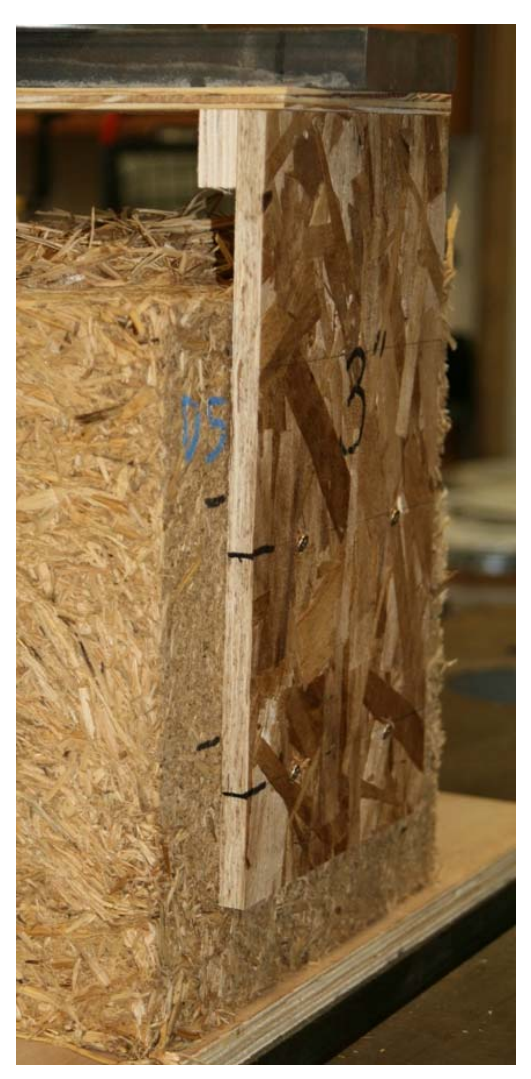

Figure 3-29: Screw Testing Slippage

\subsubsection{Screw Testing Discussion}

This investigation into shear fasteners to attach plywood sheathing to the straw block walls showed that the screws do in fact provide a significant amount of shear resistance. Although it is much softer and weaker than nails in wood, this is to be expected with the drastically different densities. Also, this shear resistance has now been quantified and can be used going forward with construction detailing and the spacing of fasteners. Seeing as the additional length is advantageous, with the costs being basically the same between long and short screws of the same type, the longer 3 inch screws will be used going forward in this report. 


\subsection{Shear Interface Investigation}

Integral features of the straw blocks used in this report are the interlocking studs on each block with the male end on top and a female end on bottom. These pegs or studs rise above the block surface $3 / 4$ of an inch and surround the cylindrical voids to interlock with each other and provide a resistance to shear along this block-to-block interface. The dimensions of these interlocking pegs were outlined in Chapter 1.

Without any prestressing forces holding the blocks together, this interface would have minimal shear resistance; but with the prestressing forces applied, this detail will provide considerable resistance. This interlocking will transfer shear between blocks, but at the foundation and top plate, mechanical fasteners will need to accomplish this transfer. The two fastening methods used in wall construction were $16 \mathrm{~d}$ nails spaced at 4 inches on each face of the block and Simpson ${ }^{\circledR}$ Mending Plates (MP36) spaced at 24 inches on each face of the block. The nails were driven through the $2 x$ sill or top plate and therefore penetrating the straw block one inch while the plates were screwed in four corners to the sill plate and the 136 teeth penetrated the block $3 / 8$ of an inch. Figure 3-30 shows the Simpson ${ }^{\circledR}$ Mending Plates used in construction.

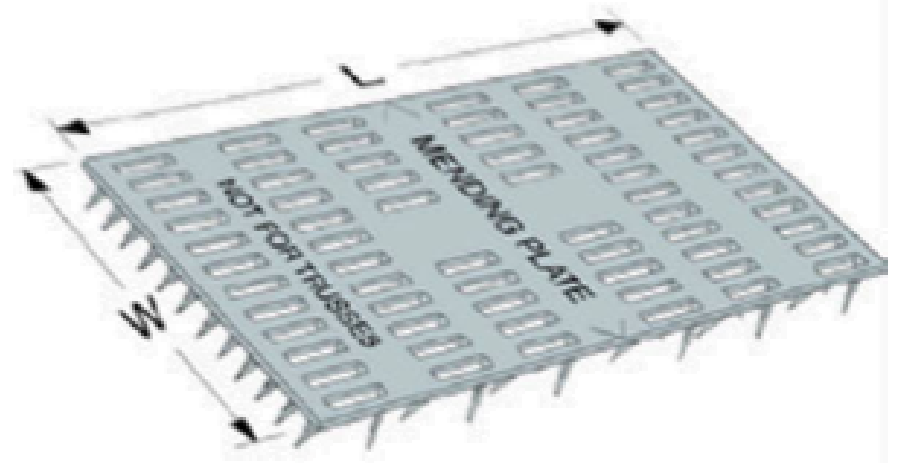

Figure 3-30: Simpson ${ }^{\circledR}$ MP36 


\subsubsection{Testing Setup}

In order to investigate whether one of these details performs better than the other, two mockups were made to simulate one foot of wall construction. A half block was sandwiched between $2 \frac{5}{8}$ inch tall top section and bottom section. Then a 14 inch long section of $1 \frac{1}{12}$ inch $x 12$ inch Douglas Fir Lumber was attached on each side using the nails or plates. A length of $1 / 2$ inch diameter thread bar was then slotted through the assembly and was prestressed to approximately 1,000 lbs. A simulated prestressed block interface specimen used in testing is shown below in Figure 3-31.

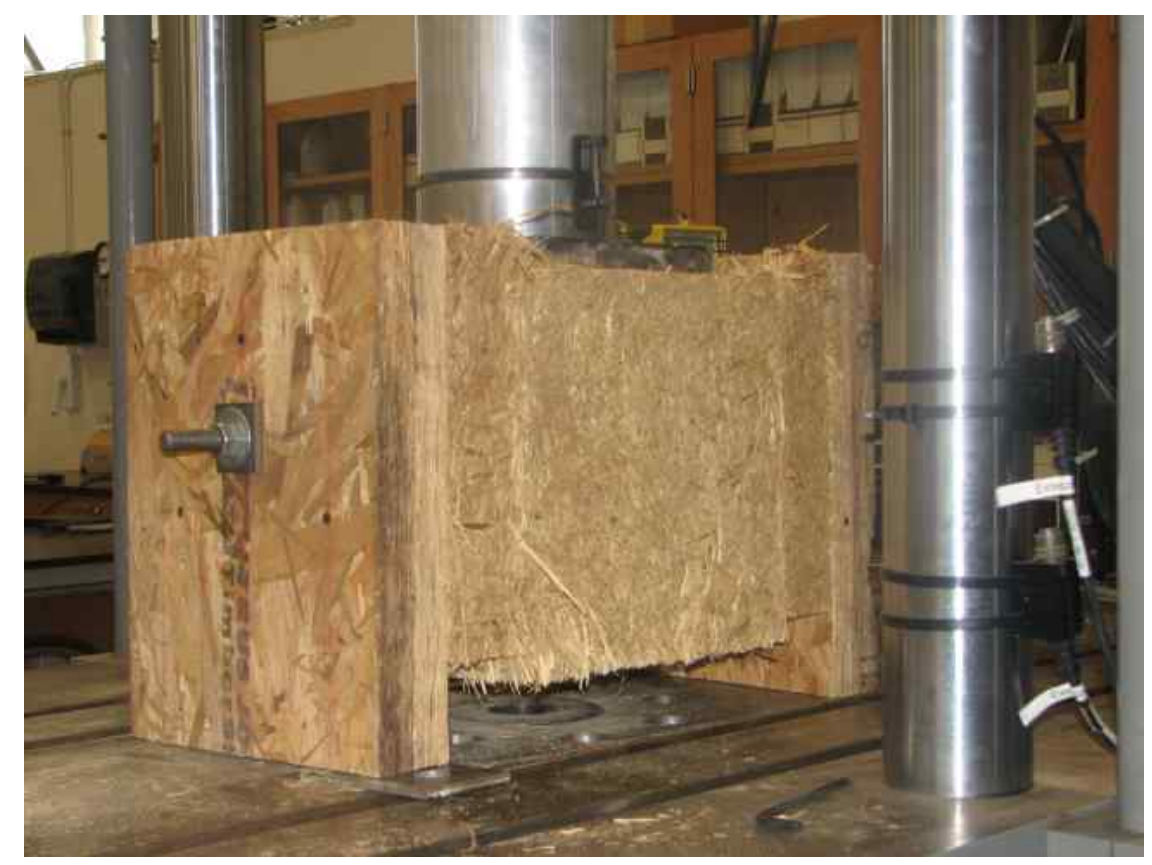

Figure 3-31: Shear Interface Test Setup 


\subsubsection{Comparison of Block-Block to Block-Wood Interface Shear Transfer}

Each specimen was placed in the same MTS frame used in prior testing and loaded at a rate of 0.25 inches per minute until considerable slippage occurred along one or more of the shear interfaces. The results from this testing are shown below in Figure 3-32 and Table 3-22.

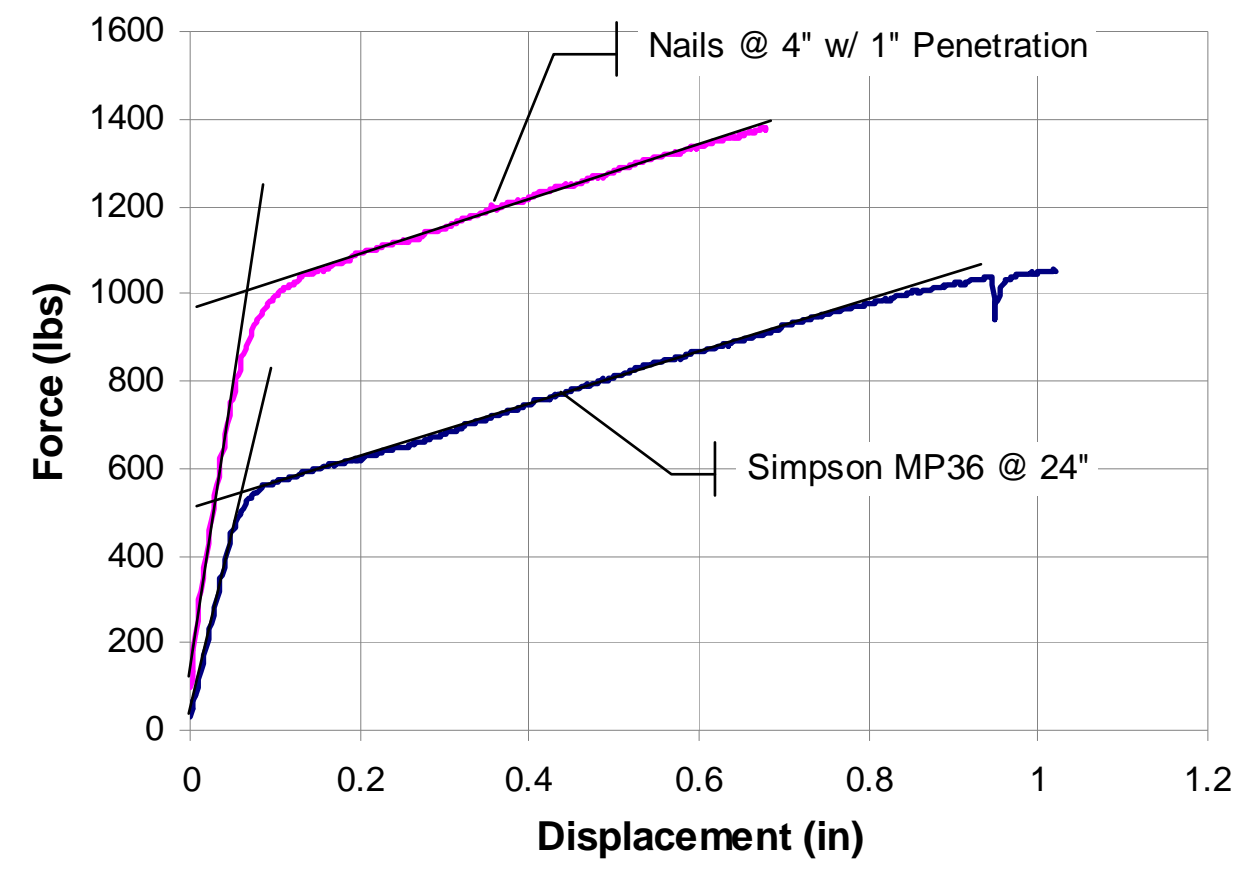

Figure 3-32: Shear Interface Testing Results

Table 3-22: Shear Interface Testing Results

\begin{tabular}{cccc} 
Fastener & $\begin{array}{c}\text { Initial Slope } \\
(\mathrm{lb} / \mathrm{in})\end{array}$ & $\begin{array}{c}\text { Yield Force } \\
(\mathrm{lbs})\end{array}$ & $\begin{array}{c}\text { Post-Yield Slope } \\
(\mathrm{lb} / \mathrm{in})\end{array}$ \\
\hline \hline Nails @ 4" & 12836 & 1010 & 614 \\
Plates @ 24" & 8637 & 554 & 596
\end{tabular}

A yield point was estimated at the intersection of the linear approximations of the two clearly different stiffnesses seen in testing. Although the above data seems to show that the nail detailing performed better than the mending plates, this was not the case. In 
both cases, all the slippage occurred during testing between the interlocking straw blocks and not at the mechanical fasteners. This can be seen in Figure 3-32. This fact was corroborated by the considerable post-test damage of the straw male and female interlocking studs.

While the initial slopes of the force-displacement curves are different between the nails and the plates, after the yield point the slopes are nearly identical (within 3\%). This indicates that after the straw-straw interface begins to fail and slip, the shear resistances provided by the mechanical fasteners were nearly identical.

If the plates were in fact weaker, then there would have been noticeable slippage at the yield point; instead, it was the straw studs that failed. As mentioned before, the test specimens were prestressed to approximately 1,000 lbs. The tightening for both specimens was done by the author, who had prestressed all four shear wall specimens, and was calibrated by "feel”. It appears that the amount of shear resistance between blocks is entirely dependent on the downward prestressing force and this is likely the cause of variations between the two specimens. The average shear resistance measured between both tests was roughly 780 lbs. Since the wall is 12 inches wide, this can be translated to a shear resistance in pounds per lineal foot.

Another important finding of this test was that the mending plates and nails both were stronger than the block interface. Although they may not have been exactly the same strength, the weak point of the shear resisting system is the straw-straw boundary and not the straw-wood transfer. 


\section{Chapter 4 Testing of Full-Scale Shear Walls}

Following the materials investigation presented in Chapter 3, four full scale shear wall specimens were constructed and tested. Each wall specimen was 8 feet wide and nominally 8 feet tall. A basic description of each of the four walls tested is found in Table 4-1.

Table 4-1: Shear Wall Testing Program

\begin{tabular}{ccc} 
Specimen & Description & Prestressing \\
\hline \hline Wall 1 & Bare Straw Block Wall & Thread Bar \\
Wall 2 & $1 / 2 "$ Sheathing Both Sides & Thread Bar \\
Wall 3 & $1 / 2$ " Sheathing One Side & Wire Rope \\
Wall 4 & $1 / 2 "$ Sheathing One Side & Thread Bar
\end{tabular}

The size of wall specimen ( 8 feet by 8 feet) was determined by an appropriate floor-to-floor construction height and keeping a 1:1 aspect ratio. Because of the exploratory nature of this study using a completely new building material, the construction and testing process included many design and detailing iterations with refinements being incorporated with each wall. 


\subsection{Laboratory Setup for Shear Wall Testing}

Laboratory testing equipment included an 80 kip capacity MTS hydraulic actuator with a +/- 10 inch displacement capacity fixed to a reinforced concrete "strong wall" as well as a moveable mock foundation anchored to a "strong floor". The tops of the walls were restrained from out-of-plane displacements simulating wall boundary conditions of a framed building structure. Between the testing of Walls 2 and 3 the laboratory facilities underwent an upgrade and the restraint system was slightly modified to provide easier specimen setup and construction. Both configurations, illustrated below in Figures 4-1 and 4-2, were deemed to provide the same out-of-plane resistance and the change did not affect testing results.

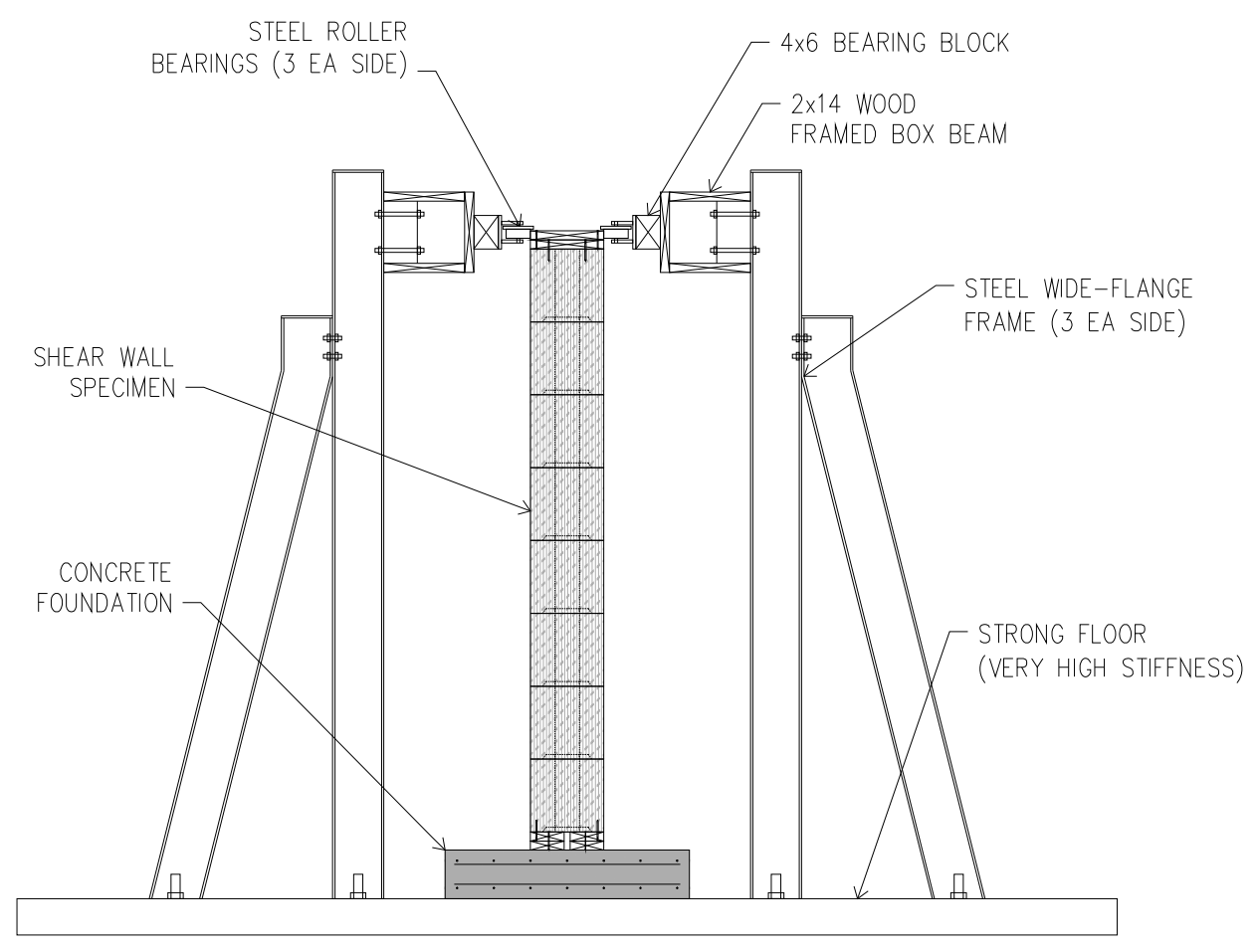

Figure 4-1: Laboratory Setup, Shear Walls 1 and 2 


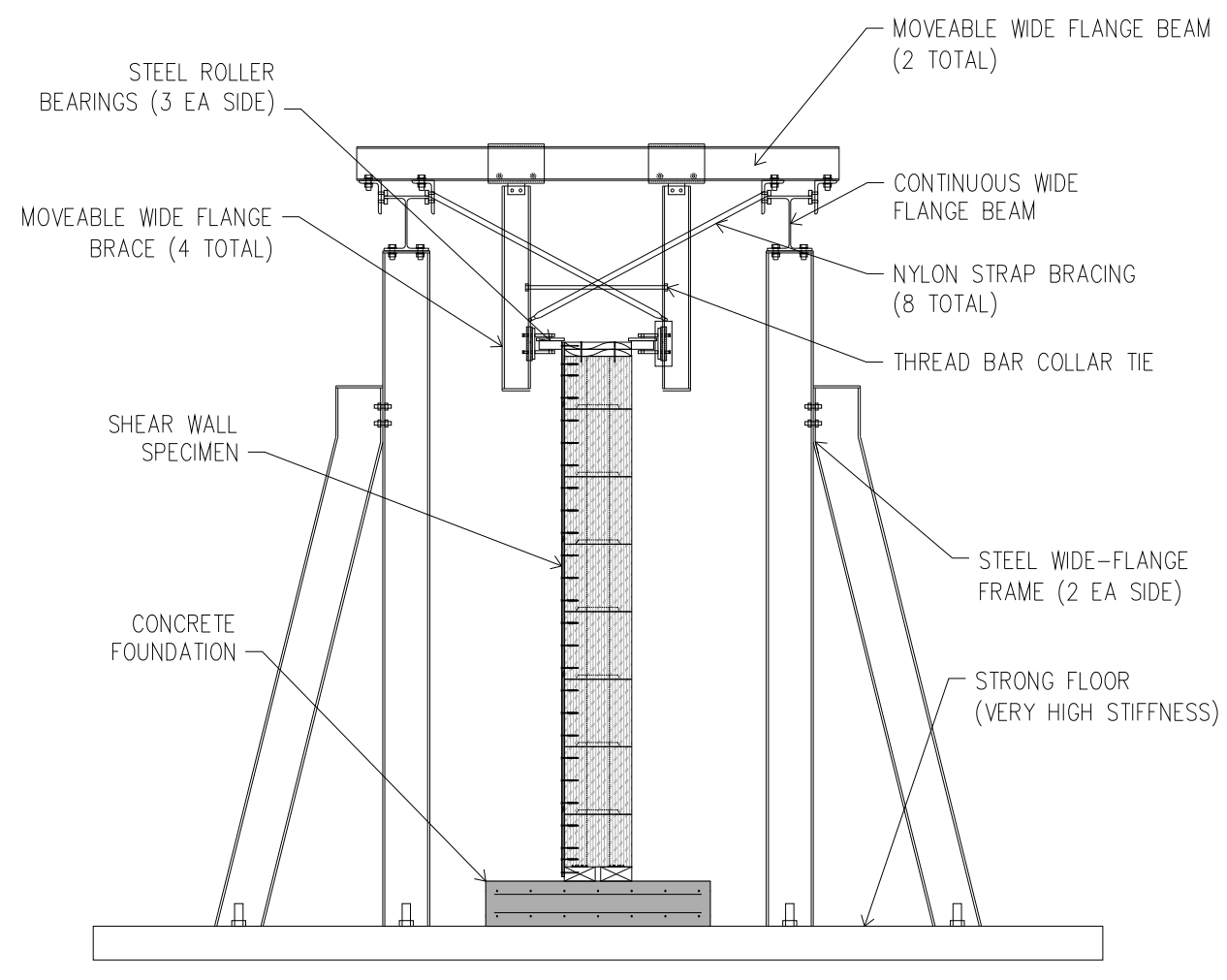

Figure 4-2: Laboratory Setup, Shear Walls 3 and 4

In both cases, the out-of-plane support of the walls was facilitated by steel wide flange braces attached to the strong floor. In the former laboratory setup (Figure 4-1), the force was transferred from the wall to steel rollers into wood box beams into the steel braces. The latter setup (Figure 4-2) used the same steel rollers attached to movable steel braces attached to a wide flange beam that rested on the original steel braces. Both systems replicated adjacent floor or roof framing in construction that would the shear wall from lateral and torsional buckling.

In all cases, the hydraulic actuator was attached to the shear wall along the drag strut, or top plate, with 20 Simpson ${ }^{\circledR}$ SDS screws. The screws were long enough to transfer load directly from the actuator to the straw blocks below the top plate. The 8 " thick concrete moveable foundation was poured on site and was fitted with the necessary 
anchor bolts (Figure 4-3) to attach sill plates. This foundation also had $3 / 4$ inch anchor bolts along its centerline spaced at 12 inches to attach the prescribed prestressing in the manner detailed later.

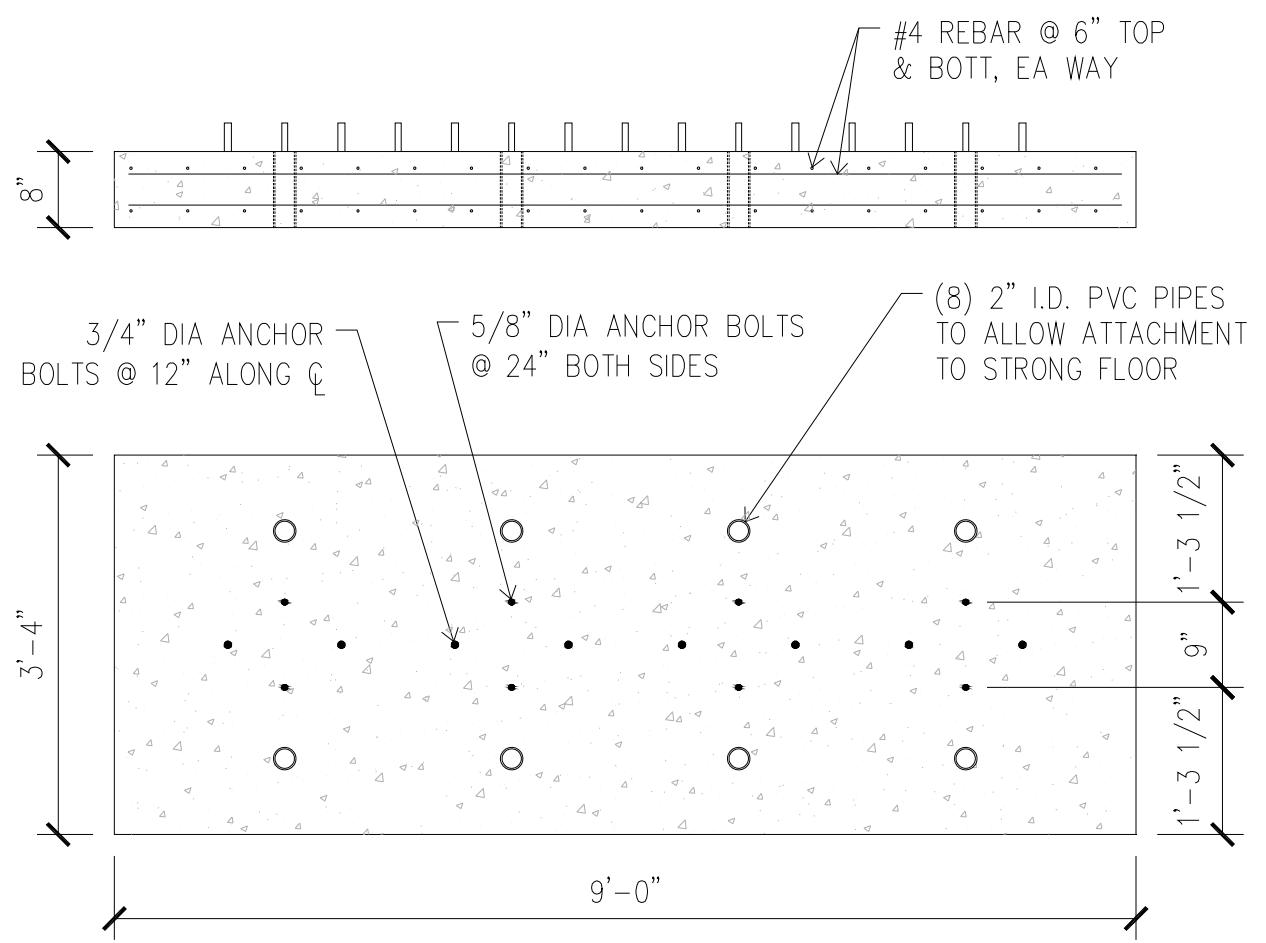

Figure 4-3: Moveable Foundation Detail

Each shear wall specimen was constructed on the foundation starting with the sill plates pre-drilled for the $5 / 8$ inch diameter anchor bolts. The 2 inch inner diameter PVC pipes allowed for $1 \frac{1 / 2}{2}$ inch bolts to be attached to the strong floor below.

\subsubsection{Wall Instrumentation}

Data collection during the testing of the straw block shear walls included instrumentation of each wall to record various deformation modes and construction detailing affects. Measurement devices included draw wire Displacement Transducers (DTRs and DTOs with "R" and "O" referring to different lengths of draw wires, 
identified by Red and Orange tape respectively) to capture large displacements, Linear Variable Differential Transformers (LVDTs) for low deformations only, and Load Cell Washers (LCWs) to measure the forces within the prestressing before and during testing. The DTRs and DTOs utilized in this study have an approximate resolution of 1/100 inch, while the LVDTs have a resolution of 1/1000 inch. The LCWs measured compression between the tightening nuts and the top plate and had a capacity of $30,000 \mathrm{lbs}$ and an approximate resolution of $100 \mathrm{lbs}$. All transducers were calibrated to an accuracy of better than $1 \%$ of the measurement. Figure $4-3$ summarizes the instrumentation orientations and locations for each wall specimen.
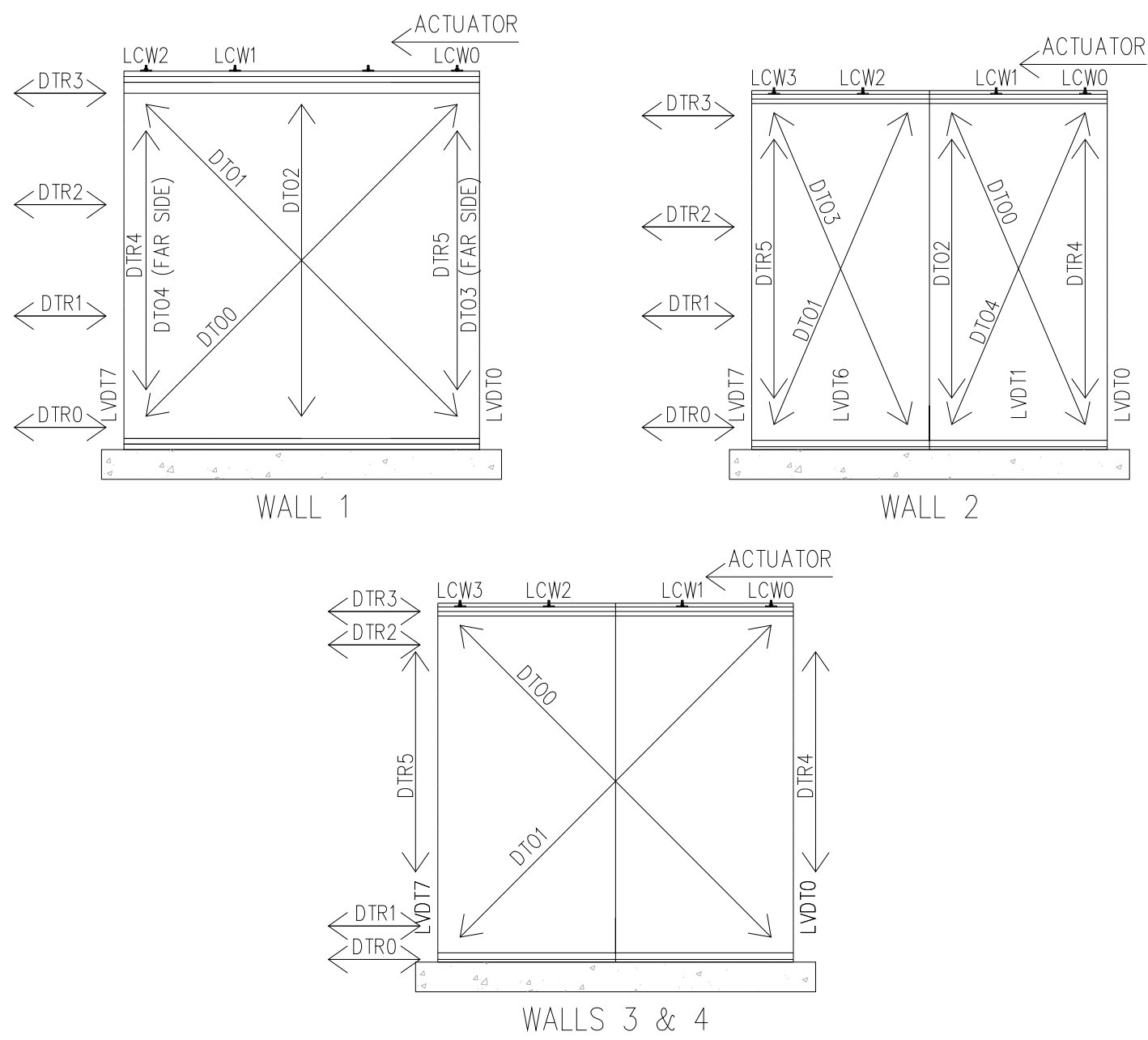

Figure 4-4: Shear Wall Measurement Instrumentation 
The first two walls tested were instrumented in a slightly different manner than the last two due to differing wall construction details and the investigation of design iteration effectiveness. The vertical DTRs and DTOs measured compressive deformation of the wall on the ends and midpoint. The diagonal DTOs were in place to measure any panel shear deformation versus rotation. The horizontal DTRs were attached to a rigid instrument "tree" and the end of the wall at uniformly distributed heights for the first two tests and just at the top and sill plates to measure differential slip between wood and straw block for the last two tests. All tests had LVDTs placed on both ends to measure uplift.

\subsection{Full-Scale Shear Wall Testing Protocol}

For the full scale shear wall testing, the CUREE-Caltech loading protocol, a pseudo-static displacement input was used (Krawinkler et al, 2001). This method of testing is a sequentially increasing, but fully reversing, cyclic displacement protocol and is used to simulate the dynamic loading of a building in lieu of shake table testing to provide force-displacement relationships for a tested structural system. Although this protocol was developed for a wood framed wall project, it was shown to simulate the energy and excitation associated with a design-level seismic event (probability of exceedence of $10 \%$ in a 50 year period). The protocol was deemed appropriate for this study because the target market and structure size for Stak Blocks is one- and two-story residential construction. Using a widely adopted method loading protocol will allow the findings of this report to be compared to a similarly constructed and tested stud framed wall. Table 4-2 and Figure 4-5 illustrate the CUREE-Caltech loading protocol. 
Table 4-2: CUREE-Caltech Loading Protocol for a 97 inch Tall Wall

\begin{tabular}{|c|c|c|c|}
\hline Cycle Type & \# of Cycles & Deflection (in) & \% Drift \\
\hline Initiation & 6 & 0.073 & \\
\hline Primary & 1 & 0.109 & $0.11 \%$ \\
\hline Trailing & 6 & 0.082 & \\
\hline Primary & 1 & 0.146 & $0.15 \%$ \\
\hline Trailing & 6 & 0.109 & \\
\hline Primary & 1 & 0.291 & $0.30 \%$ \\
\hline Trailing & 3 & 0.218 & \\
\hline Primary & 1 & 0.437 & $0.45 \%$ \\
\hline Trailing & 3 & 0.327 & \\
\hline Primary & 1 & 0.582 & $0.60 \%$ \\
\hline Trailing & 2 & 0.437 & \\
\hline Primary & 1 & 1.019 & $1.05 \%$ \\
\hline Trailing & 2 & 0.764 & \\
\hline Primary & 1 & 1.455 & $1.50 \%$ \\
\hline Trailing & 2 & 1.091 & \\
\hline Primary & 1 & 2.183 & $2.25 \%$ \\
\hline Trailing & 2 & 1.637 & \\
\hline Primary & 1 & 2.910 & $3.00 \%$ \\
\hline Trailing & 2 & 2.183 & \\
\hline Primary & 1 & 3.638 & $3.75 \%$ \\
\hline Trailing & 2 & 2.728 & \\
\hline Primary & 1 & 4.365 & $4.50 \%$ \\
\hline Trailing & 2 & 3.274 & \\
\hline Primary & 1 & 5.093 & $5.25 \%$ \\
\hline Trailing & 2 & 3.819 & \\
\hline Primary & 1 & 5.820 & $6.00 \%$ \\
\hline Trailing & 2 & 4.365 & \\
\hline Primary & 1 & 6.548 & $6.75 \%$ \\
\hline Trailing & 2 & 4.911 & \\
\hline
\end{tabular}




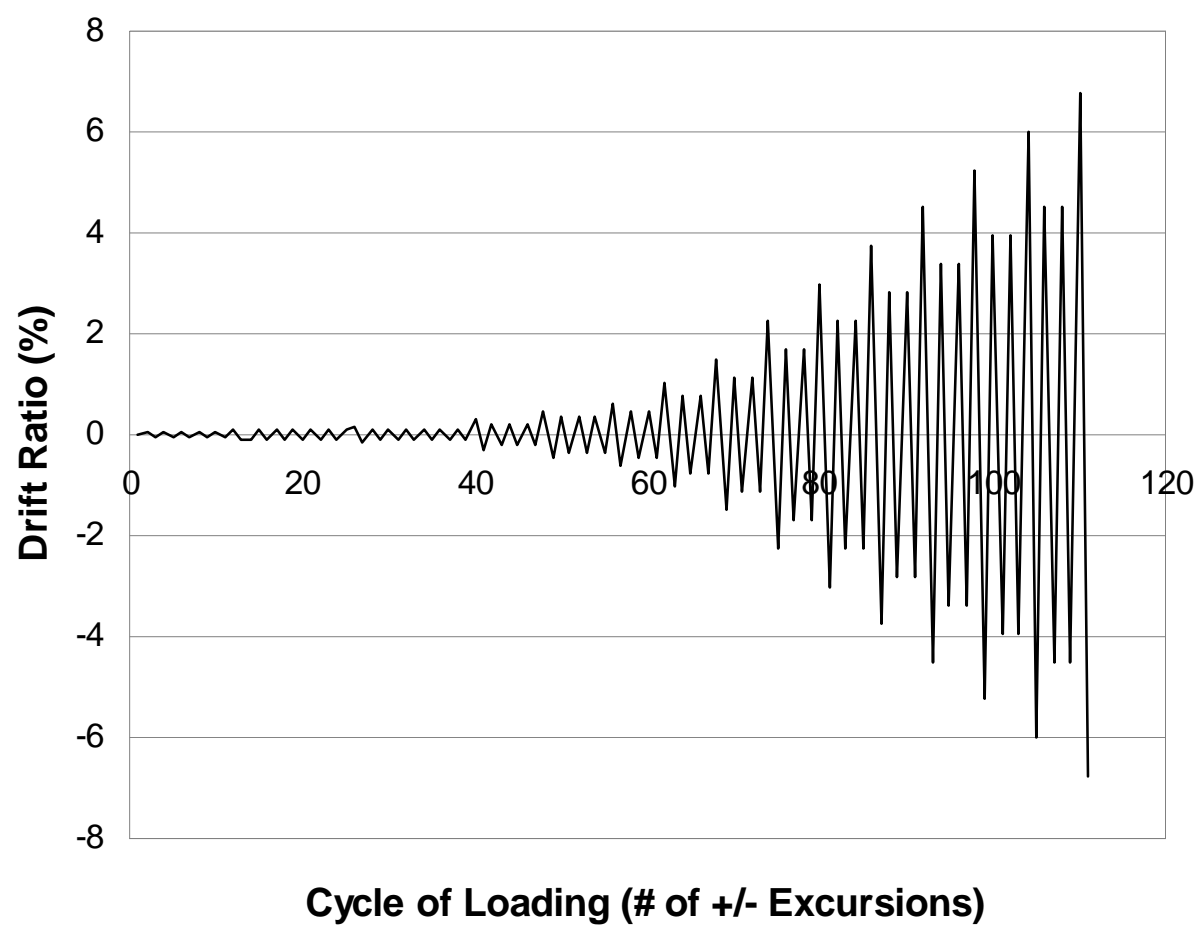

Figure 4-5: Full CUREE-Caltech Loading Protocol

In Table 4-2 above, the wall specimen was 97 inches tall so the percentage drift values are relatively close to the deflection in inches. The initiation cycles are the reference deformation based on $2.5 \%$ drift multiplied by 0.03 . Each primary cycle has a prescribed percentage drift that is higher than any displacement up to that point. The trailing cycles are all $75 \%$ of the previous primary cycle's deflection.

\subsection{Wall Prestressing}

\subsubsection{Decision to Prestress}

Prior to the construction and testing of the first shear wall specimen, the only understanding of the straw blocks and how they would behave was based on a simple materials investigation. Because of the findings of this testing and the presence of the 
interlocking studs on each block, the decision to investigate the feasibility of prestressing was made.

The idea of prestressing the blocks was based on three facets of the straw block material. First, the cylindrical voids that were a result of the manufacturing process could serve a functional purpose. A common idea or comment when first seeing the blocks was to fill the voids with reinforced concrete. This would in effect make the straw blocks insulating concrete forms (ICFs) surrounding tall and slender concrete columns. With the already existing ICF industry and the fact that the straw would then not be load bearing, this idea was scrapped, but is still recommended for testing investigations with this material. Instead of conventional straw bale where steel bars were stabbed between blocks, the prestressing tendons would pass through these voids and work with the straw to create a potentially effective structural system.

Second, prestressing the Stak Block wall will ensure that the thread bar, or prestressing tendon material, would always be in tension. This is accomplished by applying a pretension force such that even after losses have occurred, service loads applied to the structural system will be less than the prestressing force. Additionally, the materials investigation found an initial settlement toe (see Section 3.1.2) in each of the blocks tested. Although this toe reduces with cyclic loading over the life of a structure, it is a significantly softer region compared to the linear elastic zone. This toe region was typically seen up to loads of approximately 650 plf and typical residential loads can be expected to fall below this point. Prestressing a straw block wall well past this toe and into the linear elastic range would mean the block material would not undergo any initial settlement. In prestressing a straw block wall, the blocks would be pre-loaded on a 
material level and any superimposed loading on the wall would just reduce the tensile forces within the prestressing rods. For simplicity purposes as well as the fact that it falls at the beginning of the linear elastic region, a target prestressing force of $1,000 \mathrm{lbs}$ per lineal foot was selected.

Third, from a standpoint of global stability, the prestressing would provide a direct line of overturning resistance from the top plate to the foundation. This stability concern was first noticed in building the very first wall. As the blocks are placed on top of each other and the wall reaches heights nearing 8 feet, the wall tends to lean out of plane. Different methods to counteract this behavior were attempted such as alternating the position from which blocks were lifted into place and strapping around the wall and tying off to the adjacent steel frame, but neither worked effectively. Only after tightening down the prestressing nuts did the wall have sufficient stability. In future construction the placement of the floor or roof on top of the wall would aid in this process. From a load path standpoint, this stability provided by the prestressing allows for an overturning moment to be formed to resist lateral loads. Without prestressing or tensile-carrying members, the exterior skins (stucco with mesh or plywood panels) would have to transfer loads to the sill plate and foundation. The prestressing simply provides a simpler and more direct load path.

Additionally, if the blocks are to be load bearing and no prestressing was applied, the sustained dead load from the roofing or flooring would cause significant long term creep of the stack block. Either the wall would shorten, or the axial load would be transferred to the stucco, plywood, or sheet rock. Using prestressing, the strains in the height direction of the wall will not change, instead the Stak Blocks "relax" and the 
internal stresses decrease, and the stress in the threaded rod decreases simultaneously.

Furthermore, as additional load is imposed on the wall, the stresses within the straw block wall will not change (as the blocks will not shorten) instead the force in the threaded rods will decrease. It is for these reasons that the threaded rod prestressing force is selected to impart about 1,000 plf so the rods would still be in tension after the dead loads are applied to the walls and relaxation of the Stak Blocks has occurred.

\subsubsection{Wall Finite Element Analysis}

Using the computer analysis tool RAM Advanse (Bentley Systems, 2009), a simple two dimensional Finite Element Model (FEM) of a wall specimen was created. Material properties obtained in testing such as the elastic modulus, yield and ultimate strengths were input in defining values for the straw block material. Figures 4-6 and 4-7 show the exaggerated deflected shape of the wall as well as the load distribution. For visual reference, a 6 inch square grid is displayed and the cells in the blocks are numbered 1 through 8 with cells 4 and 5 being the middle two voids. The material properties assumed in the development of the model are listed in Table 4-3.

Table 4-3: Material Properties Used in FEM

\begin{tabular}{cccc} 
& $\sigma_{y}$ & $\mathrm{E}$ & $v$ \\
Material & psi & psi & \\
\hline \hline Straw Block & 20 & 900 & $0.3^{\star}$ \\
Wood & 900 & $1.60 \mathrm{E}+06$ & 0.4
\end{tabular}

The Stak Block properties were determined from initial testing and the Poisson's Ratio was taken from Bou-Ali's (1993) research on plain straw bales. Although this report did not measure a Poisson's Ratio for the straw blocks, from what was observed in 
testing, this value is very conservative. The values for the Douglas-Fir lumber are given as allowable code values in the NDS manual (American Forest and Paper Association, 2005).

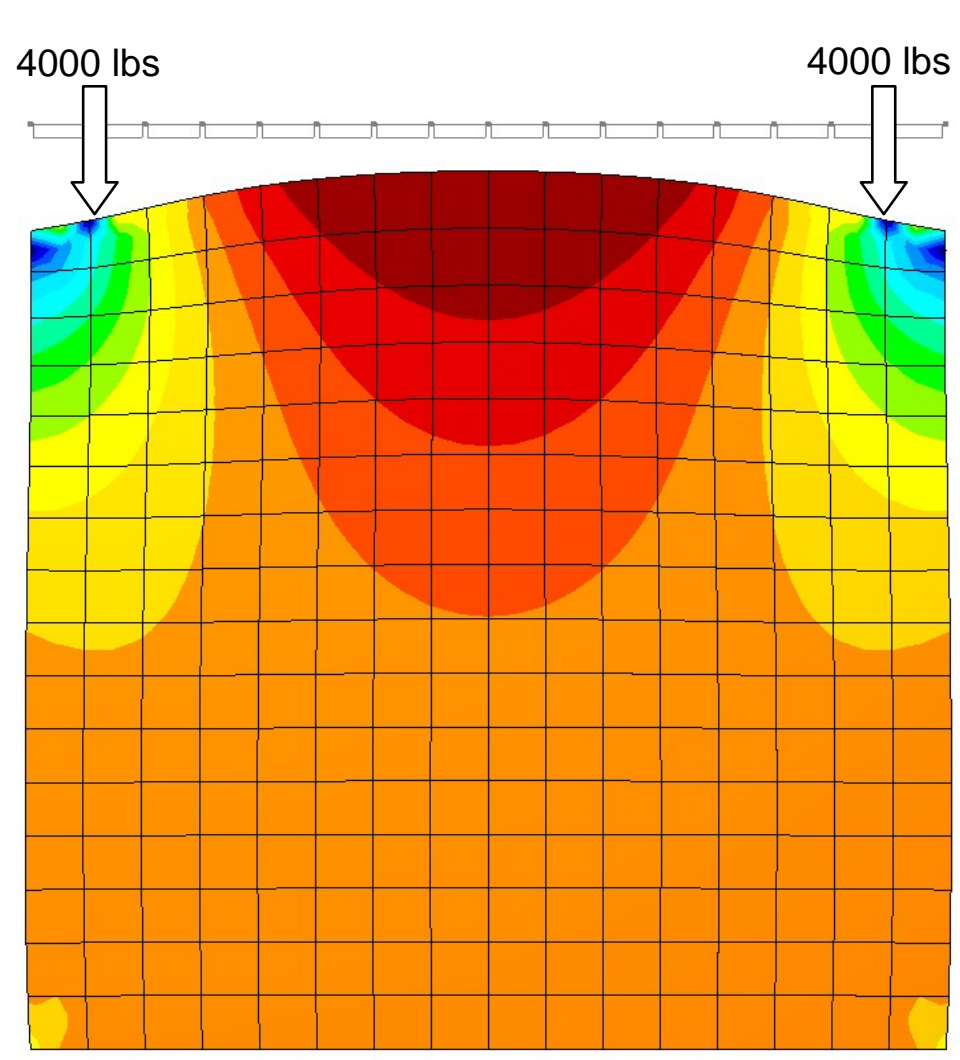

Figure 4-6: FEM with Single Top Plate Loaded at Ends Only 


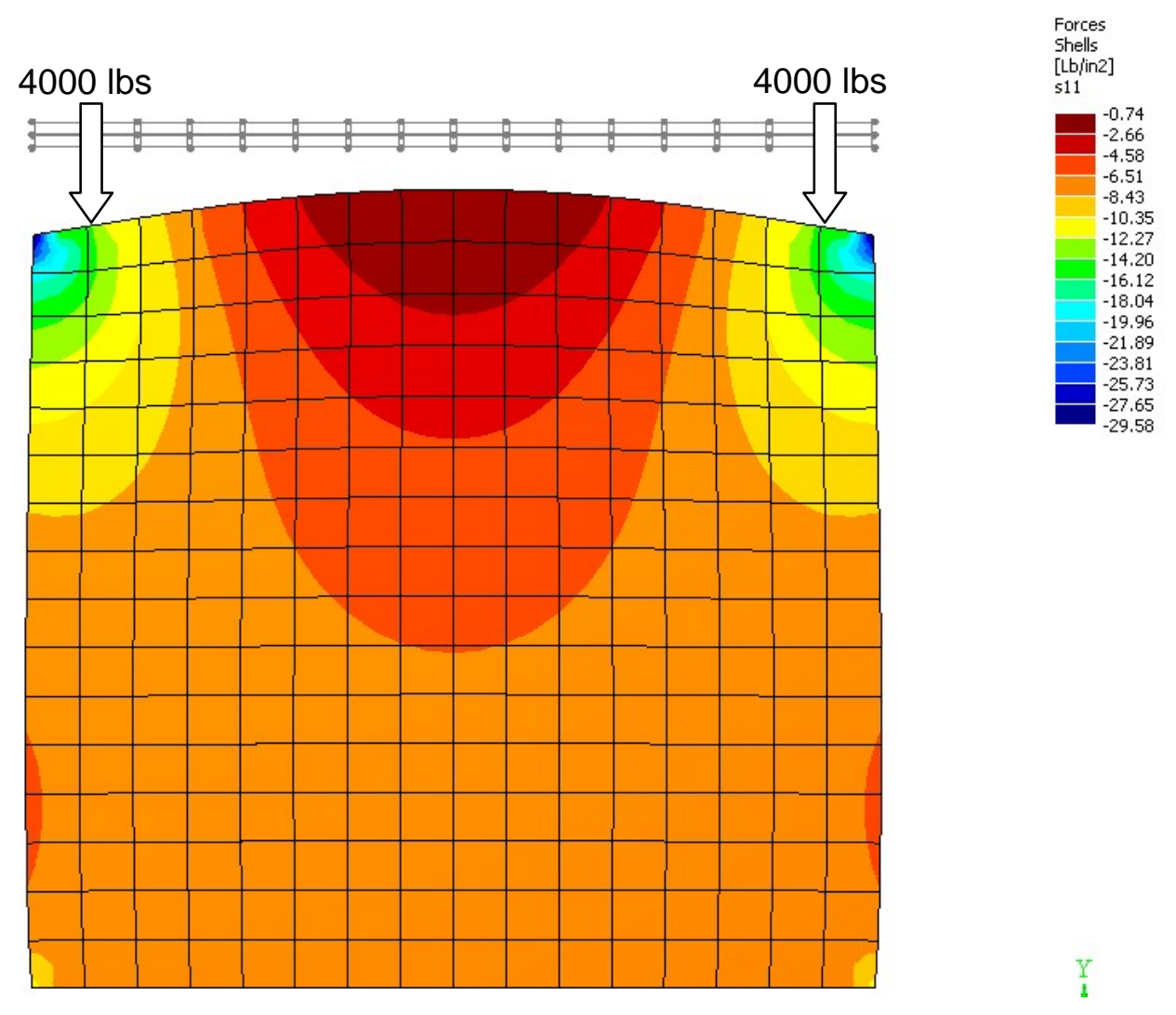

Figure 4-7: FEM with Double Top Plate Loaded at Ends Only

In both Figures above, a Douglas-Fir Larch No. 2 Grade top plate was modeled on top of the straw block wall. In the former figure the plate was only a single $2 x$, while the latter had a double top plate modeled. Because of the much stiffer material of the lumber top plate compared to the straw block, tension was actually developed near the middle of the top of the wall in Figure 4-6. Because of this modeling exercise, it was determined that a double top plate would be necessary to evenly distribute the prestressing loads.

Figures 4-8 and 4-9 below show the same Finite Element Model as above with the 1,000 plf prestressing distributed differently. 


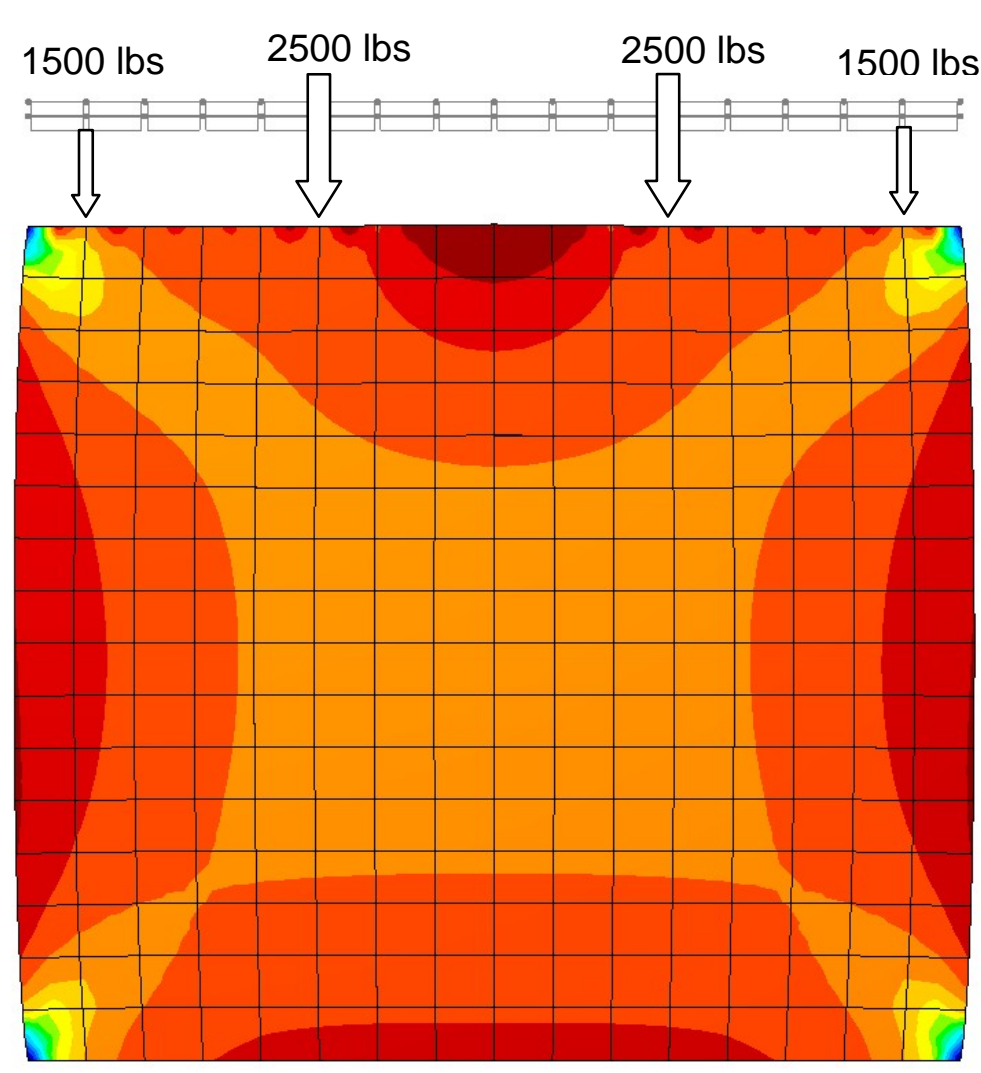

Figure 4-8: FEM with Loading at Four Points 


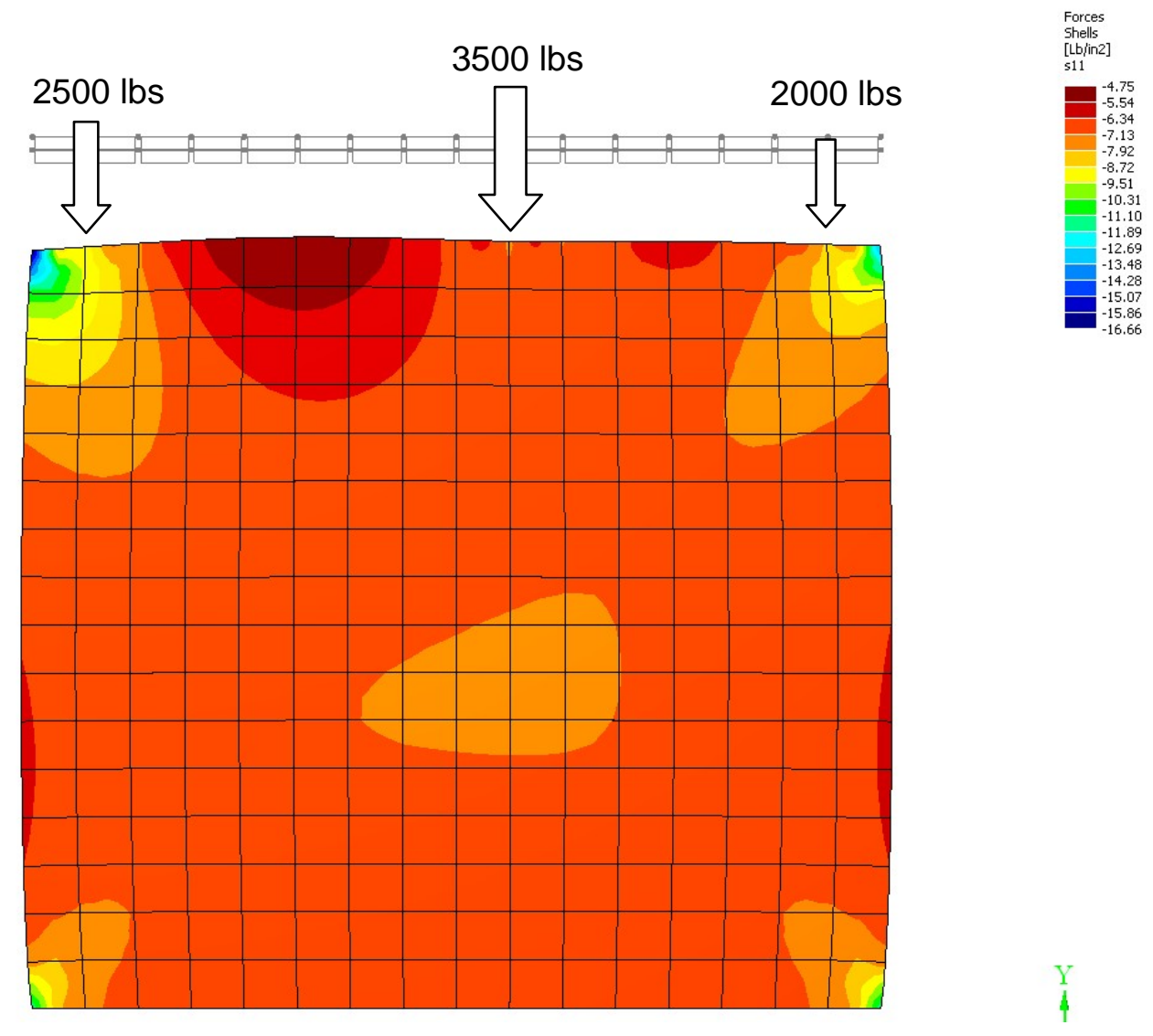

Figure 4-9: FEM with Loading at Three Points

It can be seen that although the loading at three points provided a more even distribution of force, the fact that no prestressing could be placed at the exact midpoint of the wall means there is asymmetry which is not desirable. The loading at four points (Cells 1, 3, 6 and 8) is optimal in that the 1,000 plf prestressing was accomplished without having to use every cell. This fact is further illustrated by the calculated deflections from the Finite Element Model shown in Table 4-4.

Table 4-4: FEM Calculated Deflections at Top of Wall

\begin{tabular}{cccccc} 
Load Case & $\Delta_{\max }(\mathrm{in})$ & Location & $\Delta_{\min }(\mathrm{in})$ & Location & Range (in) \\
\hline \hline Sgl Top PL, Ends & 0.96 & @ Cells 1 \& 8 & 0.38 & @ midpoint & 0.58 \\
Dbl Top PL, Ends & 0.85 & @ Cells 1 \& 8 & 0.45 & @ midpoint & 0.4 \\
4 Points & 0.65 & @ Cells 1 \& 8 & 0.64 & @ midpoint & 0.01 \\
3 Points & 0.69 & @ Cell 1 & 0.61 & @ Cell 3 & 0.08
\end{tabular}


The range values in the table above quantify the differential displacement potential in each prestressing method. The most effective method for providing uniform loading and displacement is the four-point loading shown in Figure 4-8. Both load cases where just the ends were prestressed have large differential displacements $(0.4$ inches $)$. Based on this study, during construction of the actual shear walls, the threaded bars were placed in four voids with the pretensioned forces and locations shown in Figure 4-8.

\subsection{Wall 1 Testing}

\subsubsection{Wall 1 Configuration}

Based on materials testing and a basic Finite Element Analysis, it was decided that for the first test a bare wall (no OSB sheathing) would be constructed using $1 / 2$ inch diameter threaded rods in cells $1,3,6$ and 8 . The $1 / 2$ inch (specimen $1 / 2 " \mathrm{~A}-1$ ) was chosen over the $5 / 8$ inch because of the results from materials testing that showed yield forces ranging from 9,850 to $12,000 \mathrm{lbs}$ (Figure 3-21 and Table 3-17); in effect, the $1 / 2$ inch threaded rods with yield strengths in excess of $50 \mathrm{ksi}$ are the same strength-wise as $5 / 8$ inch threaded rod with a 36 ksi yield strength (Grade 36). Since the forces within an individual rod were not anticipated to be greater than this range during testing, the smaller and therefore cheaper thread bar diameter was used for the first test. If the blocks performed better than expected and one of the thread bars yielded or even ruptured, this failure mode would be very ductile and not lead to imminent collapse of the shear wall.

Wall 1 was built using a double $2 \times 6$ sill plate with a row of $16 \mathrm{~d}$ nails passing through the topmost plate and the straw blocks placed on top of the exposed nail points. The wall was built to a height of 97 inches and then a double $2 \times 14$ top plate that was 
ripped down to 12 inches was placed on top with nails penetrating into the blocks in the same manner as below. The top plate was pre-drilled to allow the $1 / 2$ inch diameter allthread to pass through and be threaded onto reducing coupling nuts attached to the $3 / 4$ inch anchor bolts in the foundation. The construction detailing for the first wall specimen tested is found below in Figure 4-10.

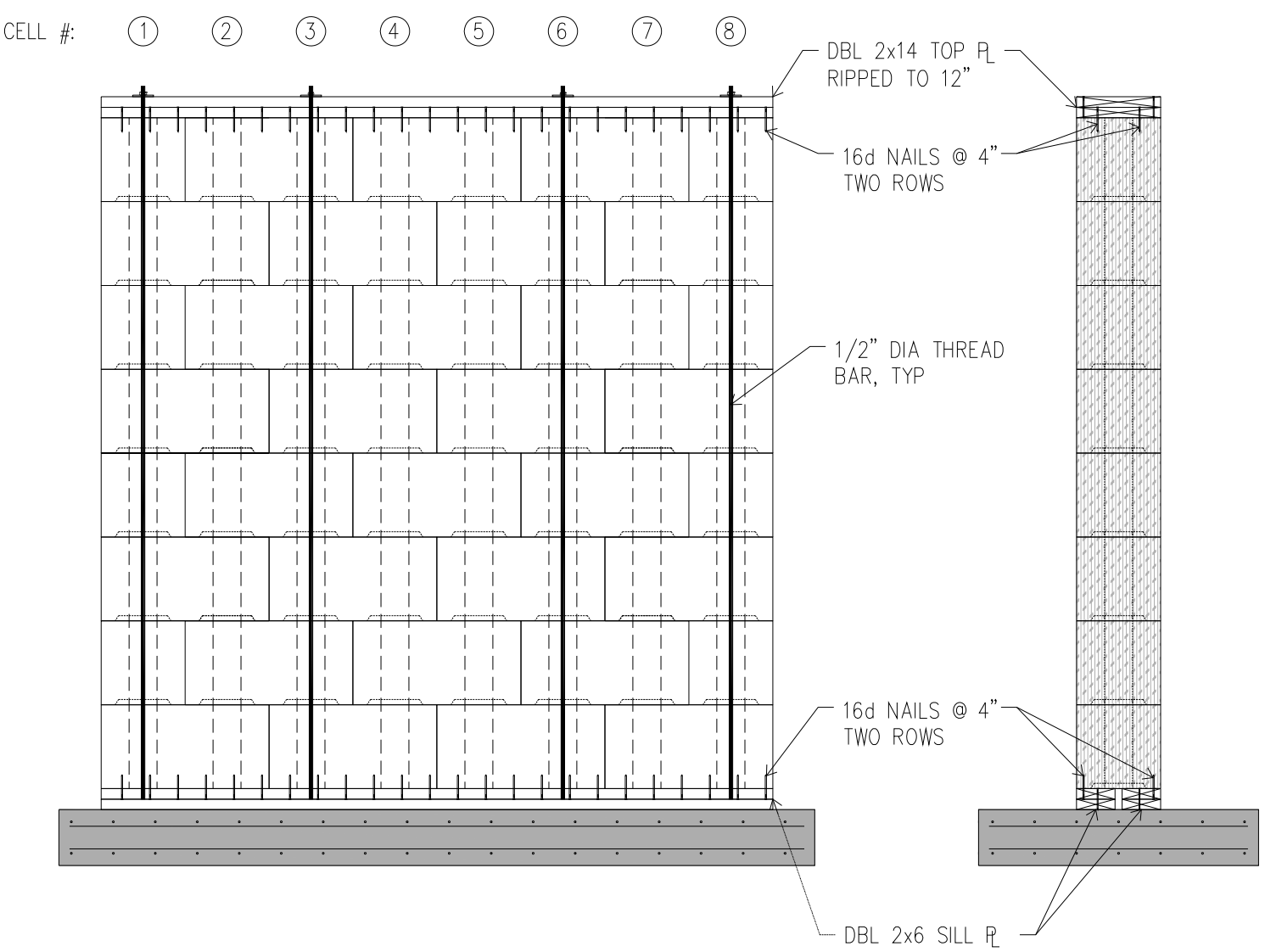

Figure 4-10: Wall 1 Details

\subsubsection{Wall 1 Testing Results}

Wall 1 was tested using the CUREE-Caltech loading protocol as described in Section 4.2. Due to cautious pessimism associated with the first test using this straw block material, the test machine actuator ran out of stroke on the positive primary cycle at $5.25 \%$ drift. Despite this fact, the bare wall never approached collapse at the very high 
drift levels. The shear force versus displacement behavior is shown below in Figure 411.

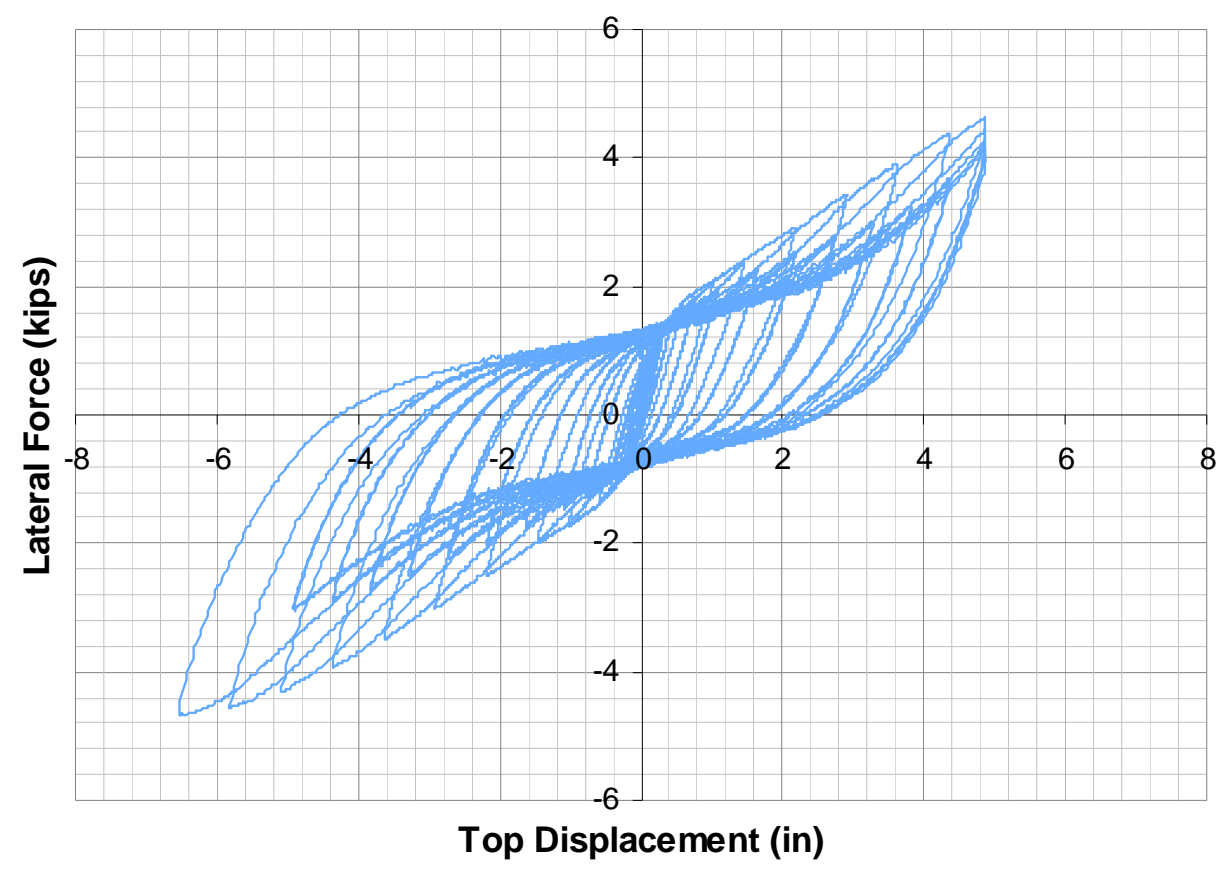

Figure 4-11: Wall 1 Testing Results

\subsection{Wall 2 Testing}

\subsubsection{Wall 2 Configuration}

Following the encouraging results from the unsheathed Wall 1, the next test was aimed to look at the opposite end of the detailing spectrum: OSB sheathing on both sides. Besides this change to "heavy" detailing, the sill plate which had been a double $2 \times 6$ for the first test was switched to a $3 \times 6$ pressure treated Douglas-Fir. The same shear transfer detail was in place at the base of the wall with $16 \mathrm{~d}$ nails penetrating the straw block through the sill plate. The nails in this case were predrilled to ensure straight driving to provide the maximum penetration depth possible. 
The top plate material and size changed for this test as well. Since the wall was a full 12 inches thick, large trees would need to be milled to cover the full thickness of the wall. While Wall 1 used 2x14's ripped down to an even 12 inches and given that this report is investigating an innovative, sustainable building material, this seemed impractical and hypocritical. Because of this, a double top plate made of $1 \frac{1 / 4}{4}$ inch by 11 7/8 inch Laminated Strand Lumber (LSL) was used. When centered on the 12 inch wide wall, the smaller LSL pieces were just $1 / 16$ of an inch shy on each face. This distance was deemed small enough to drive a nail through the sheathing to pull it flush with the top plate.

Another major change in this specimen was the actual wall height. For the first test, the straw portion of the wall ended up being 97 inches following prestressing. OSB sheets typically measure 4 feet by 8 feet and since the blocks were a full 12 inches tall, the plywood sheathing would not be able to attach to the top and sill plates. Because of this, material was removed from the top and bottom rows of blocks. $2 \frac{5}{8}$ inches were removed from both rows to create a straw block wall that was theoretically $903 / 4$ inches tall. With the double top plate and sill plate each adding $2 \frac{1}{2}$ inches of height, this gives a full wall height of nearly 96 inches when the rough, inexact dimensions of the blocks are taken into consideration. The effects of reducing the block heights both from the top and bottom are discussed in Section 3.1.10.

The $15 / 32$ inch OSB panels were attached with 3 inch long gold screws. In order to mimic typical wood framing details, the top and the sill plate nailing consisted of $10 \mathrm{~d}$ nails spaced at 4 inches. For simplicity sake, a screw spacing of 4 inches on the panel edges and 8 inches in the "field" (16 inches apart to mimic stud framing) was chosen. 
Equation 4-1 illustrates the moment resisting capacity about the centroid of a panel of the screws versus nails. The calculation is based on a simplification of the available strength of the screws versus nails, their respective location compared to the centroid of the panel, and the number of fasteners.

\section{Equation 4-1: Screw versus Nail Resistance}

$$
\begin{aligned}
& M_{\text {screws }}=[(\# \text { edge }) *(\text { dis tan ce })+(\# \text { field }) *(\text { dis } \tan c e)] * Z_{\text {ult,screw }} \\
& M_{\text {screws }}=(48 * 2 f t+24 * 0.67 \mathrm{ft}) * 201 \mathrm{lbs} / \text { screw }=22,512 \mathrm{ft}-\mathrm{lbs} \\
& M_{\text {nails }}=[(\# \text { edge }) *(\text { dis } \tan c e)] * Z_{\text {ult,nail }} \\
& M_{\text {nails }}=(24 * 4 \mathrm{ft}) * 410 \mathrm{lbs} / \text { nail }=39,360 \mathrm{ft}-\mathrm{lbs}
\end{aligned}
$$

The resisting moment of the nails spaced at 4 inches is almost twice that of the screws spaced at 4 inches on the edges and 8 inches in the field. In order to have the moments equal, the screw spacing would have to be cut in half. Having screws spaced at 2 inches on the edges and 4 inches in the field would be prohibitively expensive in terms of labor and materials. Because of this and the very ductile failure of the screws, the larger spacing was used in the construction of Wall 2. In order to fairly investigate the detailing changes mentioned above, the same size prestressing was used as the first specimen, this time using specimen $1 / 2$ " D-1 (Table 3-15). Figure 4-12 shows the Wall 2 specimen as built and tested. 


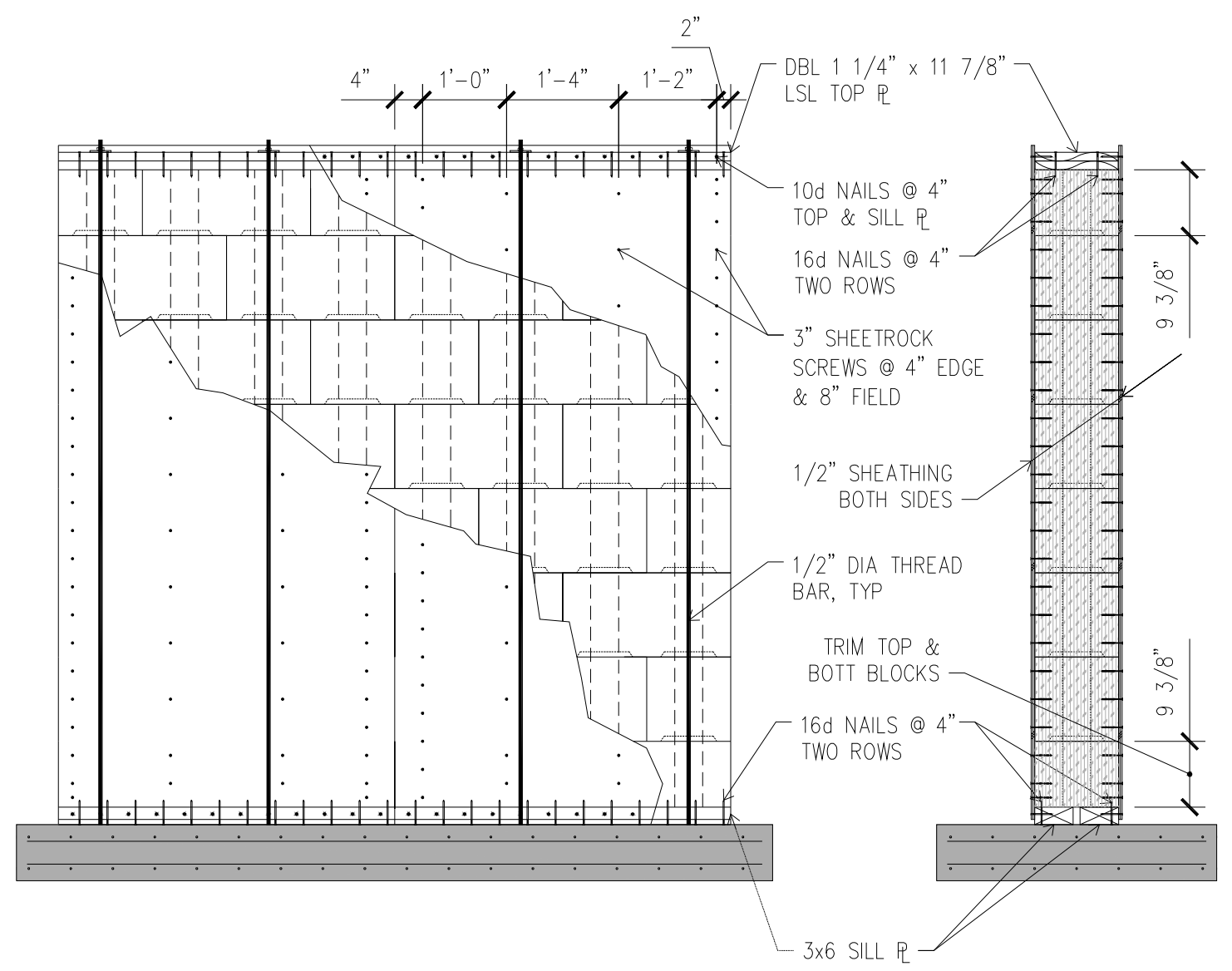

Figure 4-12: Wall 2 Detail

\subsubsection{Wall 2 Testing Results}

Wall 2 was also tested using the CUREE-Caltech loading protocol in order to investigate how the straw blocks would do with the assistance of OSB panels. As expected, the sheathing on both sides of the wall greatly increased the strength over the bare wall. The shear force versus displacement behavior is shown below in Figure 4-13. 


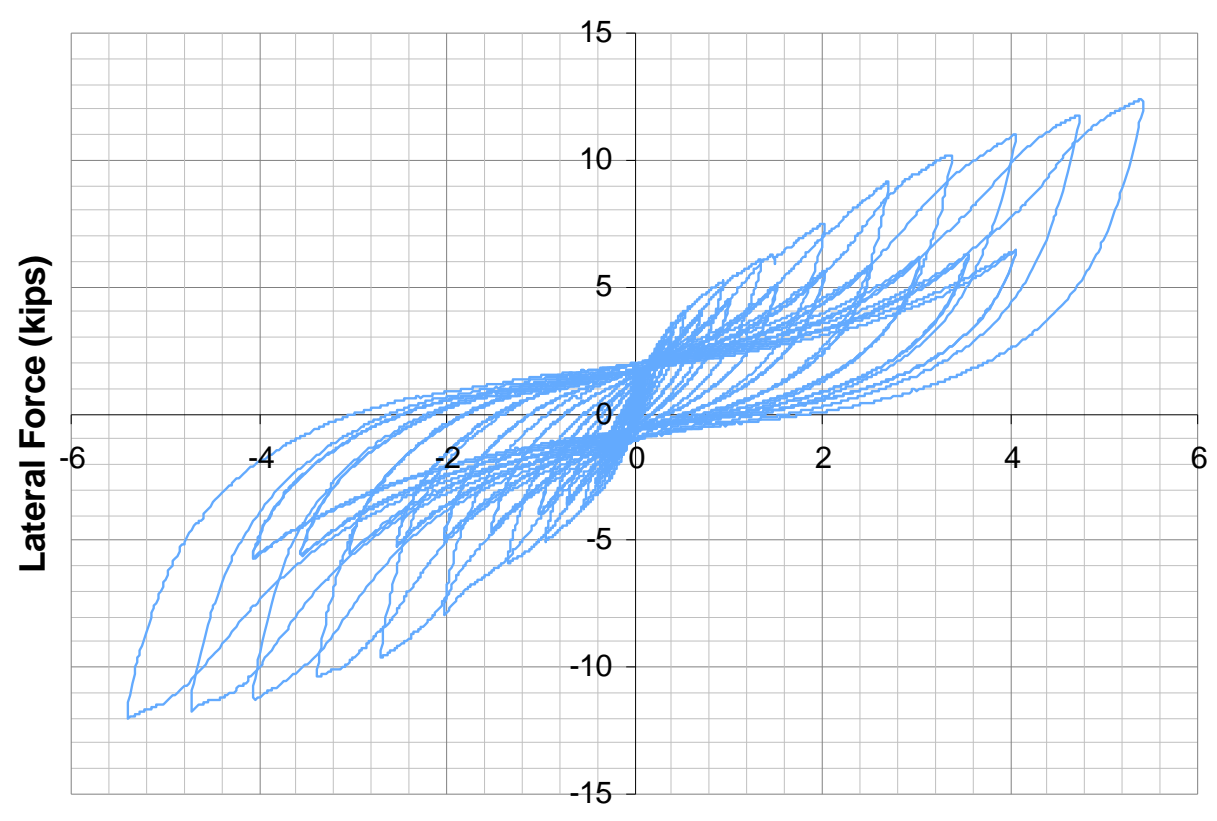

Top Displacement (in)

Figure 4-13: Wall 2 Testing Results

\subsection{Wall 3 Testing}

\subsubsection{Wall 3 Configuration}

The most common potential application or usage of the straw blocks in the future will be structural sheathing applied to one side with an architectural finish or nothing at all on the interior. Because of this, the third wall specimen was prepared with $15 / 32$ inch OSB sheathing on only one side.

Wall 3 will keep the 3x6 sill plate and double LSL top plate constant from Wall 2. Because of the presence of sheathing, the blocks were trimmed in the same manner as Wall 3. Because no major withdrawal issues occurred during the testing of Wall 2, the same nail and screw spacing was used to attach sheathing. One detailing change is the shear transfer mechanism from the sill plate to straw block. In lieu of using the nails 
driven through the sill plate, the Simpson ${ }^{\circledR}$ Mending Plates mentioned in Section 3.4 will be used with the teeth facing upward. The attachment of these is easier and simpler than the nails and they prevent much less of a safety hazard for workers than the 48 nails sticking up out from the foundation.

The most significant change in Wall 3 was the use of wire rope in place of the thread bar to provide the prestressing. With Wall 1 and Wall 2, the thread rod, instead of threading each block down 8 feet of exposed bar, the prestressing was installed once the wall was full height. This was accomplished by lowering the all-thread section down the cylindrical voids and finding the reducing coupler nut and threading tight. Although this report is focusing on the feasibility of using these blocks in construction and developing details to do so, this method would not be code-compliant. The special inspections section of the 2006 International Building Code (International Code Council, 2006) states in Section 1704.3.3.1:

"While the work is in progress... for bolts requiring pretensioning, the special inspector shall observe the preinstallation testing and calibration procedures... and determine that all plies of connected materials have been drawn together and properly snugged."

This structural observation of the thread rod being completely threaded snug to the anchor bolts could not take place because that connection would be hidden within at the time of tightening.

This constructability issue led to the use of wire rope as an alternative prestressing material. The $1 / 4$ inch wire rope was looped through an eye bolt attached to the coupling nut on the anchor bolt. Properties of the wire rope are provided in sub-section 3.2.3. As 
the wall was built up, the wire ropes were threaded through the successive blocks until the wall was at full height. The loop was pulled tight and spliced with three fasteners around another eye bolt. This top eye bolt then passed through the double top plate and was tightened to the prescribed tensile force with a nut.

Overall there were many constructability issues that came with using the wire rope to prestress. The two main ones being the difficulty in threading the eye bolt through the top plate with the wire rope loop being so tight and then the fact that even when pulled tight, the wire rope had slack to be picked up before pretensioning. Despite these problems, the wall was constructed and is detailed in Figure 4-14.

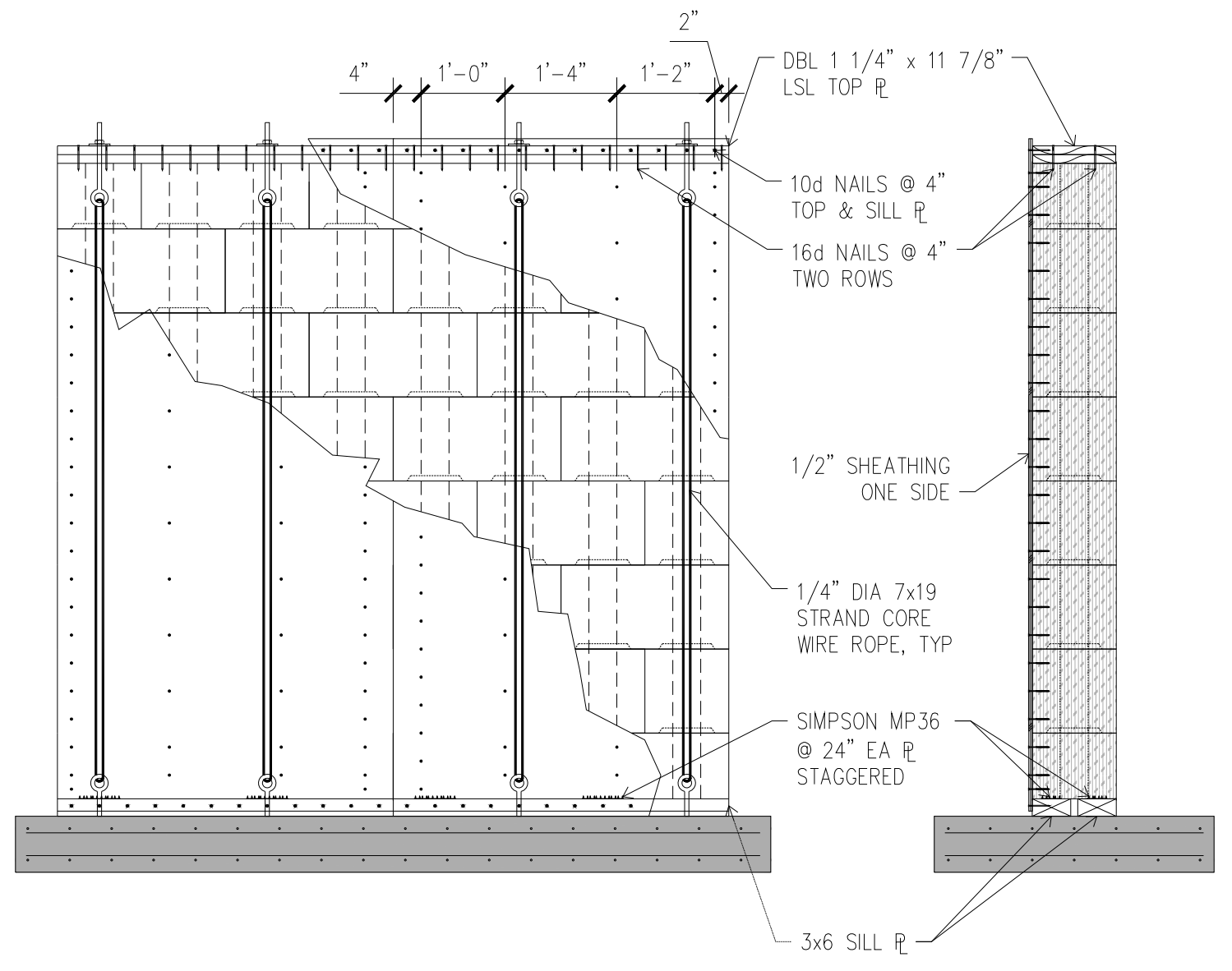

Figure 4-14: Wall 3 Detail 


\subsubsection{Wall 3 Testing Results}

Wall 3 was again tested using the CUREE-Caltech loading protocol in order to investigate how effective the cable prestressing would be. In the end, Wall 3, with sheathing on one side and wire rope prestressing, did not perform much better than Wall 1. Wall 3's peak load was only $15 \%$ higher and its load at $2.5 \%$ drift was only $20 \%$ greater. The shear force versus displacement behavior is shown below in Figure 4-15.

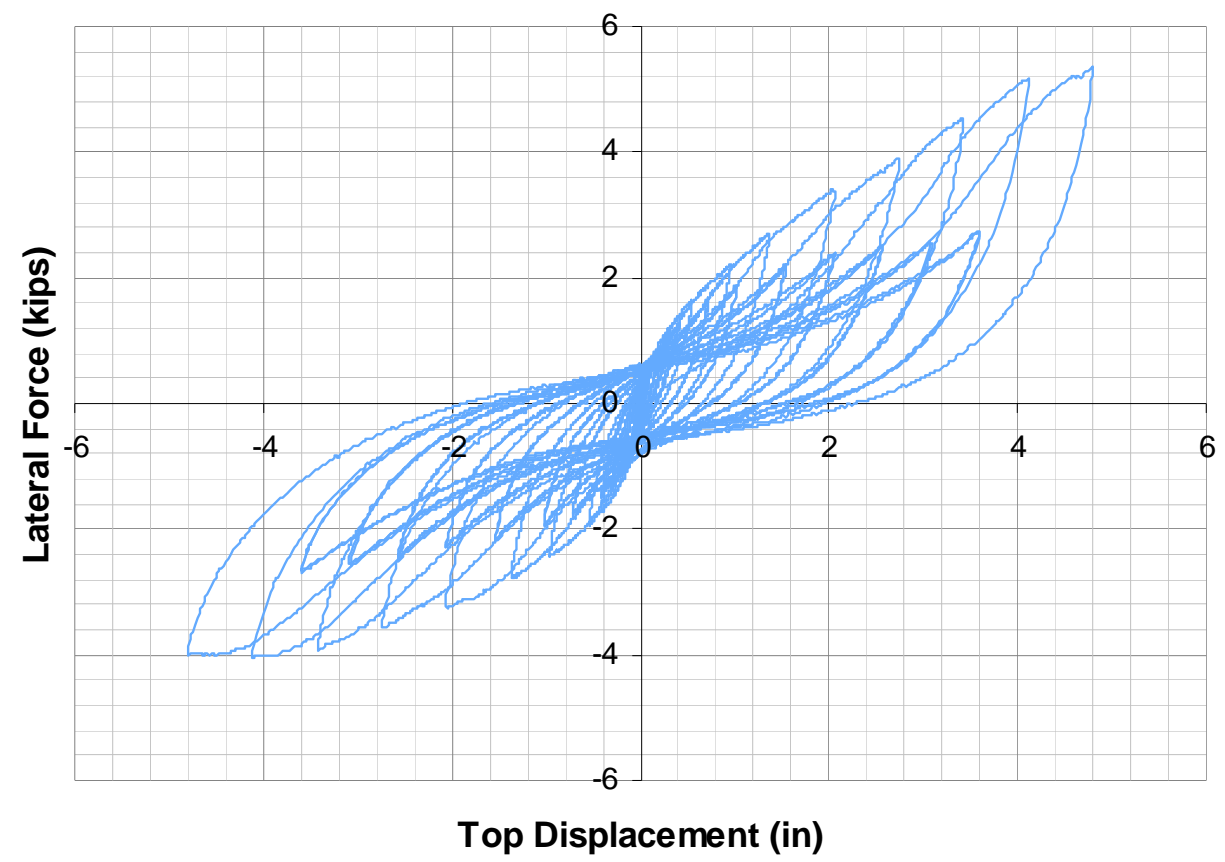

Figure 4-15: Wall 3 Testing Results

\subsection{Wall 4 Testing}

\subsubsection{Wall 4 Configuration}

Reaching the final shear wall specimen of the testing program, most detailing iterations have been investigated in prior tests. For Wall 4, the only change from the prior test was reverting back to thread rod from wire rope to provide the prestressing 
(using specimen $1 / 2 "$ D-1 from Table 3-15). Since the sheathing provided so much shear resistance in Wall 2, the results from Wall 3 were expected to fall halfway between the bare Wall 1 and the heavily detailed Wall 2. As this was not the case, Wall 4 would verify whether the sheathing on one side created a torsional irregularity and therefore a weaker wall, or if it was solely the use of wire rope.

The solution to the special inspection issue with the earlier thread bar method was to provide an inspection hole. A 3 foot 3 inch length of thread bar was attached to the foundation's anchor bolts with a reducing coupler before placing any straw blocks. It should be noted that in practice, this short length of thread bar would be drilled and epoxied into the concrete. This short length then had a $1 / 2$ inch- $1 / 2$ inch coupling nut with an inspection hole attached to its end. To allow for visual verification that the thread bars were snug together, a $2 \frac{1}{2}$ " hole was drilled with a Forstner bit halfway up the fourth block. The Forstner bit cut through the straw block quickly and cleanly and the resulting hole was cleaned out of any debris. After the height of the wall had been built, the remaining length of thread bar was lowered down to the coupling nut and tightened. A flashlight was all that was needed to see that both thread bars were butted together within the nut. The photograph in Figure 4-16 was taken to show the ease of verification. The final Wall 4 detailing is shown in Figure 4-17. 


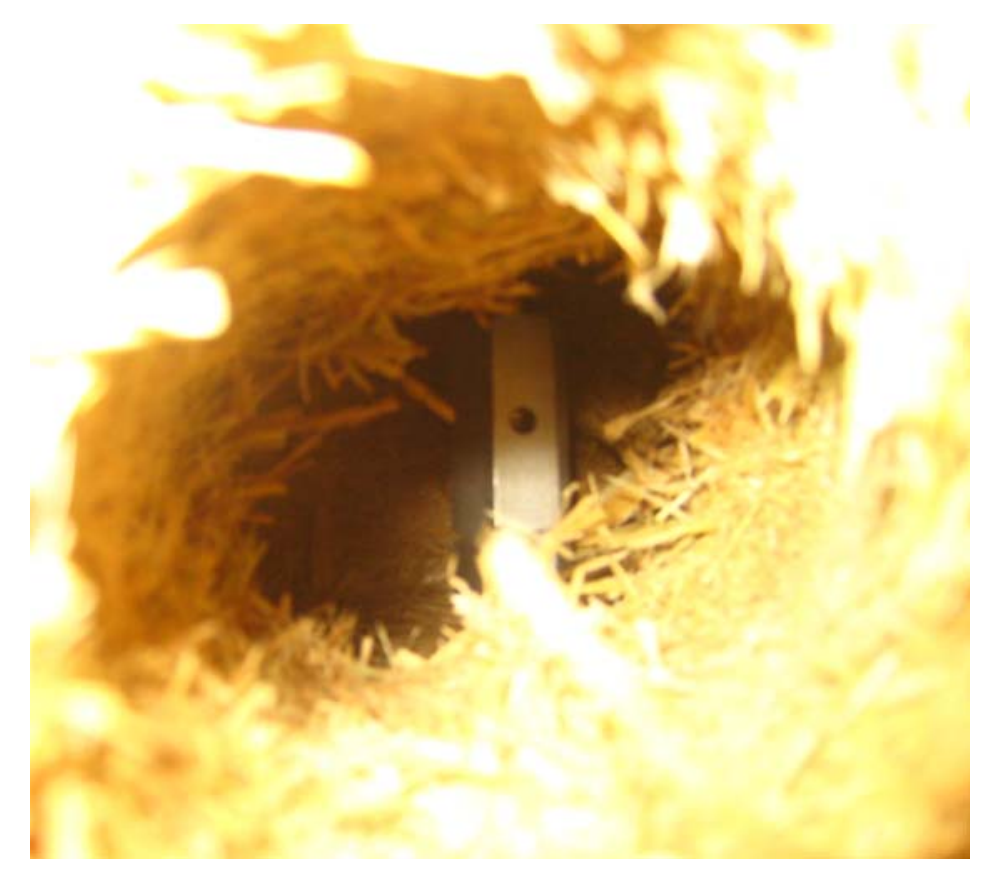

Figure 4-16: Visual Inspection Hole

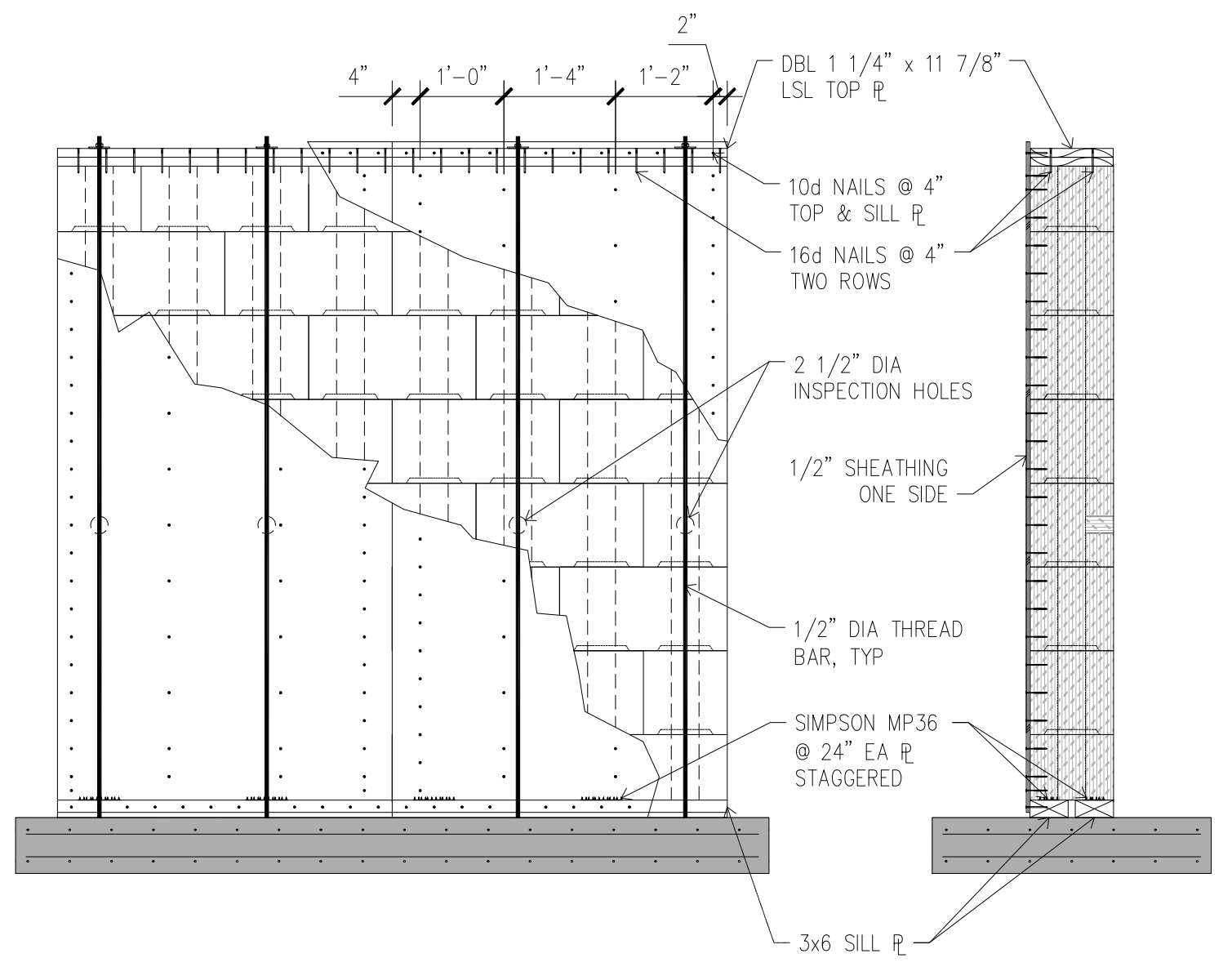

Figure 4-17: Wall 4 Detail 


\subsubsection{Wall 4 Testing Results}

Wall 4 was tested using the same CUREE-Caltech protocol and during the $3.75 \%$ drift primary cycle, out-of-plane resistance was lost in the test configuration. The problem was fixed and the reinforced test frame completed the cyclic testing at higher drifts. As expected the results fell closer to a halfway point between Walls 1 and 2 . The shear force versus displacement behavior is shown below in Figure 4-18.

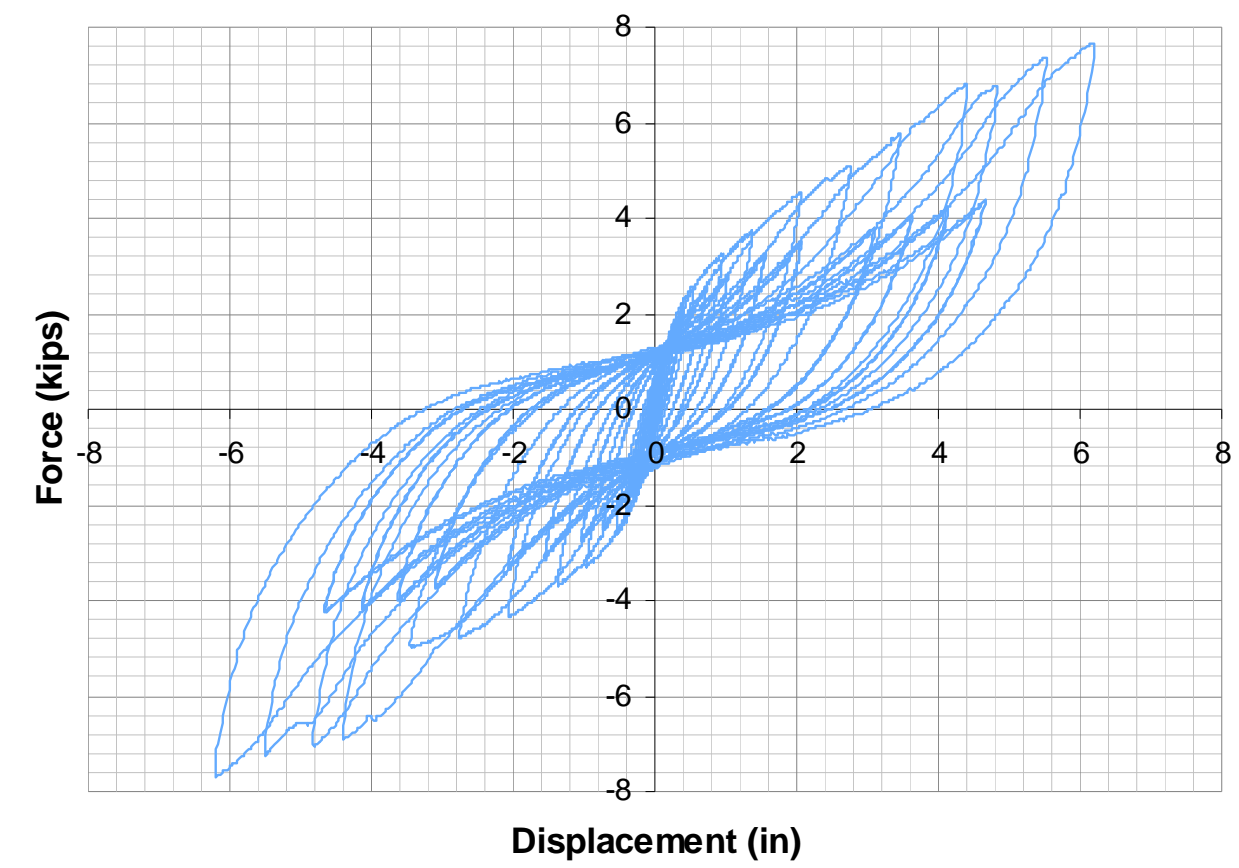

Figure 4-18: Wall 4 Testing Results

\subsection{Summary of Test Results}

Throughout the shear wall testing program various detailing iterations and improvements were implemented. In the end, Wall 1 would be the most lightly detailed, bare wall that could be used as a baseline. Wall 2 is the opposite end of the spectrum having sheathing on two sides and would be considered the heavily detailed specimen. Walls 3 and 4 are the most likely to be implemented in future construction in terms of 
shear panels and detailing, with the only difference between the two being the material used in prestressing. Table 4-5 summarizes the basic findings of all four wall tests with a more in-depth investigation into the differences in the wall behaviors following in Chapters 5 and 6.

Table 4-5: Shear Wall Testing Summary

\begin{tabular}{cccc} 
Specimen Description & $\begin{array}{c}\text { Peak Load } \\
\text { (kips) }\end{array}$ & $\begin{array}{c}\text { Drift @ } \\
\text { Peak Load } \\
(\%)\end{array}$ & $\begin{array}{c}\text { Avg. Load } \\
\text { @ 2.5\% Drift } \\
\text { (kips) }\end{array}$ \\
\hline \hline Wall 1: Bare Wall, Thread Bar & 4.69 & 6.75 & 2.94 \\
Wall 2: Sheathing Both Sides, Thread Bar & 12.36 & 6.00 & 8.89 \\
Wall 3: Sheathing One Side, Wire Rope & 5.38 & 5.25 & 3.54 \\
Wall 4: Sheathing One Side, Thread Bar & 7.69 & 6.75 & 4.72
\end{tabular}




\section{Chapter 5 Full-Scale Shear Wall Results}

As previously stated, a large part of the investigation into this new and unproven building material is the development and verification of detailing standards. The detailing iterations and construction decisions described in the previous chapter are compared and quantified from the data collected during testing in this chapter.

\subsection{Prestressing Tensile Forces during Testing}

\subsubsection{Prestressed Wall Behavior}

On a basic level, a data evaluation of whether or not the wall systems performed as expected during testing was necessary. The prestressed wall specimens should theoretically behave similarly to a prestressed concrete beam. While this analogy is not perfect, it does help to illustrate the more complex behavior of the tested walls.

For example, the lateral load acting at the top of the wall would be analogous to an imposed shear force on a beam cross section. In this case, the wall would be a beam turned on its side with the prestressing tendons (thread bar or wire rope) creating 
permanent internal stresses to balance external forces (the actuator or lateral forces due to earthquake or wind). Since the straw block is very similar to concrete in that it is strong in compression but very weak in tension, the prestressing will handle the tensile forces and allow the straw blocks to slide and move independently (i.e. "crack").

The basic load path that was expected to form during testing was the load from the actuator would transfer to the top plate, or drag strut. From here there would be shear transfer to the top surface of the straw blocks and down between the interlocking blocks to the foundation. The load path for an unsheathed, prestressed, Stak Block wall is better illustrated by the free body diagram in Figure 5-1.

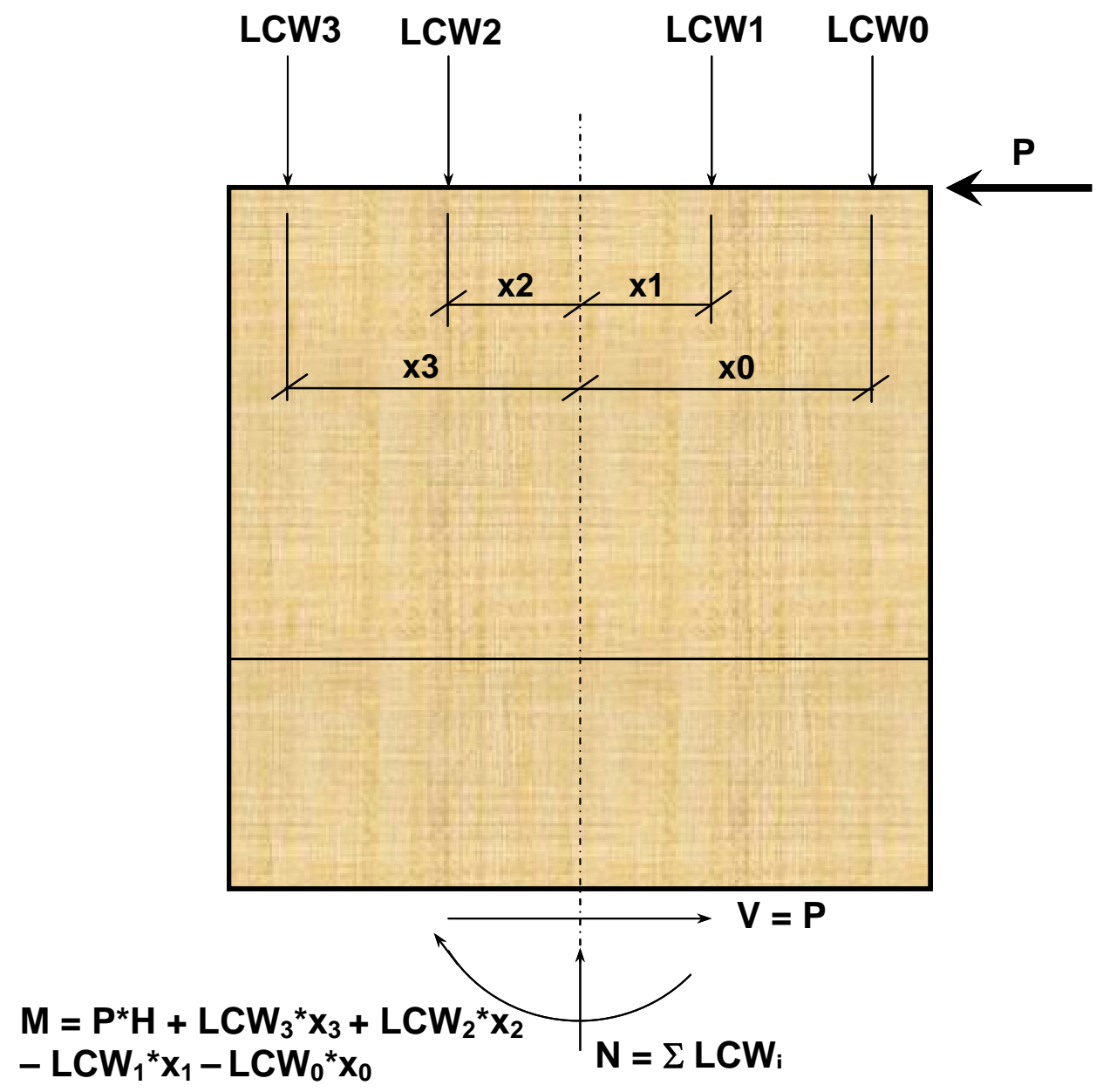

Figure 5-1: Free Body Diagram on Straw Wall 
In the figure above, the LCW forces represent the prestressing forces imposed on the wall which increase and decrease in phase with the push/pull loading from the actuator. The shear at the base of the wall that is transferred to the sill plate is equal to the horizontal actuator force, P. It is assumed that the prestressing forces balance around the centroid of the wall $(\mathrm{LCW} 3=\mathrm{LCW} 0$, etc) and the overturning moment is created by the force, $\mathrm{P}$, at a height, $\mathrm{H}$. The normal force at the base of the wall, $\mathrm{N}$, is equal to the sum of all of the LCW prestressing forces.

While this basic description of the wall system under cyclic loading simplifies the effects of three-dimensional distribution of stress, this analysis will allow for the stress on the base of the Stak Blocks to be quantified. The forces and moment shown above create a linear elastic resulting stress profile at the base of the wall. Figure 5-2 shows both of the resulting stress profiles on the bottom of the wall.
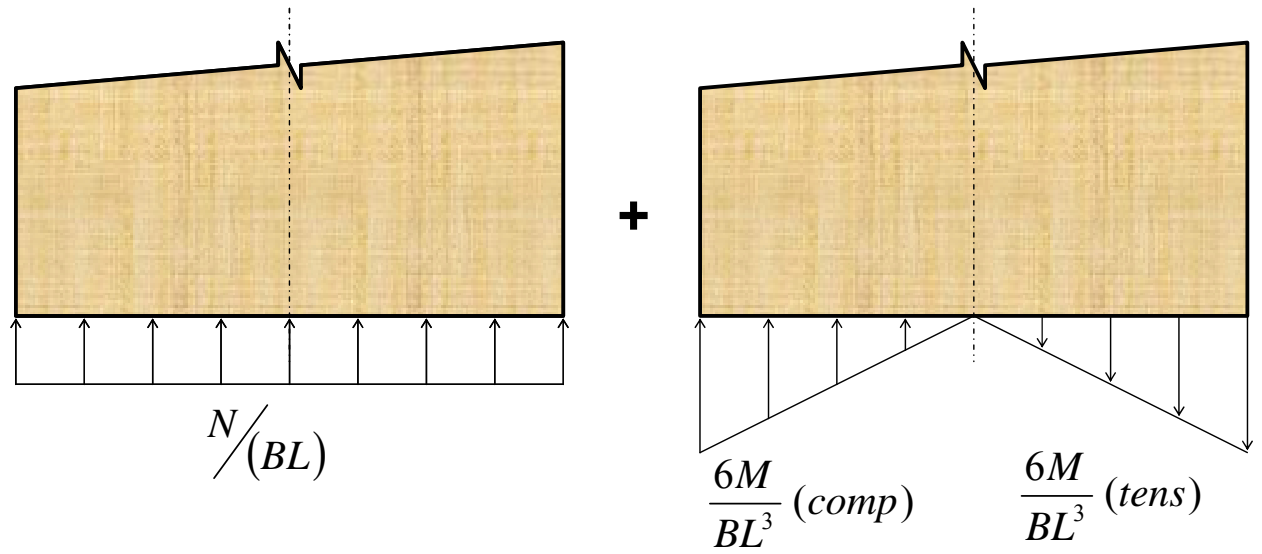

Figure 5-2: Stresses on Base of Wall

The stress $N /(B L)$ comes from the normal force resultant from the prestressing forces over an area B wide (the thickness of the wall: 1 foot) by L long (the length: 8 feet). The stresses resulting from the bending moment are simplified from a substitution 
of the moment of inertia of the wall into $\left(M^{*} C\right) / I$. Depending on the magnitude of these forces, one of the following total stress profiles shown in Figure 5-3 will be experienced by the wall.

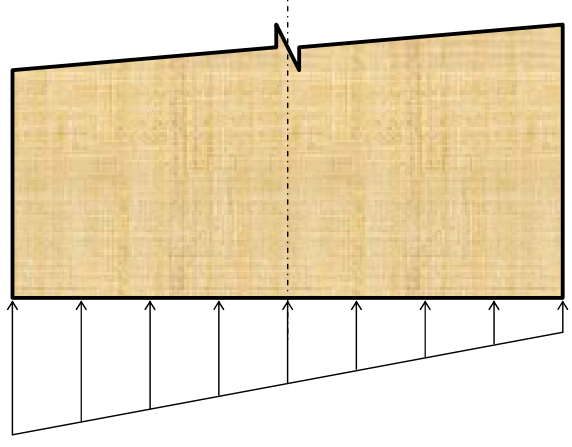

when $M \leq \frac{1}{6} N L$

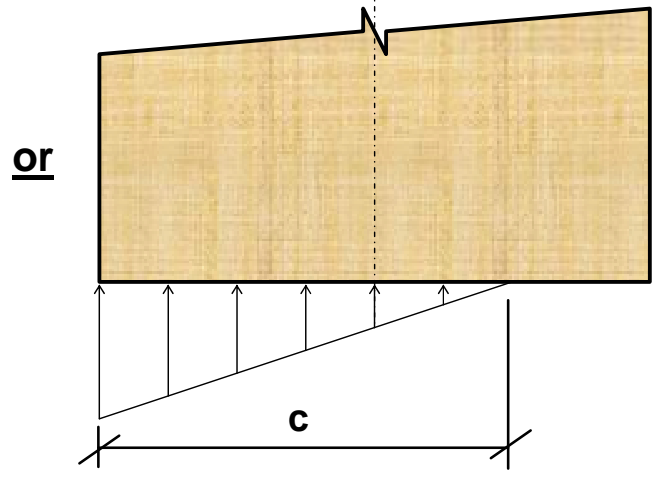

when $M>\frac{1}{6} N L$

Figure 5-3: Summing Stresses at Base of Wall

In the right hand diagram of Figure 5-3, zero stress is developed where there would be tension, as tensile stresses cannot develop between the blocks. This manifested itself with uplift of the wall seen during shear wall testing.

\subsubsection{Calculation of Block Stresses during Testing}

The data from Wall 1 testing included the tensile forces within the thread bar at every point during the cyclic loading. Using the simplifications and forces outlined in the prior section, an analysis was performed on the wall to determine the magnitude of stresses imposed on the Stak Blocks at the base. The assumption that all prestressing forces would balance would no longer be valid as the forces were constantly changing during the cyclic loading. The maximum tensile values seen in the prestressing is presented in Table 5-1. 
Table 5-1: Maximum Forces within Prestressing in Walls 1 and 2

\begin{tabular}{ccc} 
& Wall 1 & Wall 2 \\
\hline \hline LCW0 (kips) & 6.35 & 10.90 \\
LCW1 (kips) & $\sim 4.72$ & 3.56 \\
LCW2 (kips) & 3.76 & 6.00 \\
LCW3 (kips) & 5.06 & 10.66
\end{tabular}

Since the fourth load cell (LCW1) was not available for the testing of Wall 1, the value of 4.72 kips was estimated from symmetry within the wall and determining an appropriate ratio by looking at the available data from the three load cells. The yield point measured in materials testing for the type of $1 / 2$ inch diameter thread bar used was approximately $12,000 \mathrm{lbs}$ while the bars in Wall 1 only reached 6,350 lbs. It should be noted that in Wall 2's testing, LCW0 was damaged when its cable was pinched at the $4.5 \%$ primary cycle and the data it produced after this point was unreliable. Because of this issue, the maximum push cycle of 10.36 kips was used to obtain the above data immediately before the damage occurred.

Wall 2 can be interpreted as a "worst case" heavy detailing scenario in terms of the forces expected on the thread bar. Yet the thread bar still did not appear to yield during the cyclic testing. The maximum tensile force recorded actually occurred at $4.5 \%$ drift before the damage described above and greater tensile forces could have been imposed on the thread bars but were not recorded in the data. The force of 10,900 lbs is very close to the measured yield point of the bars used and is actually higher than the ultimate fracture load of the thread bar specimen $1 / 2$ " C-1. This illustrates the point that the source from which the prestressing material is obtained is very important. While the 
large forces seen were at very high drift levels (4.5 to $6 \%$ ) and the failure of a thread bar would not mean the collapse of a structure, for the design of walls that anticipate seeing higher loads, a move to $5 / 8$ inch diameter thread bar may be necessary.

By performing a statics analysis and summing the vertical forces as well as the moments around the bottom middle of the wall, it was determined that for the positive loading direction (actuator pulling) the depth of the compression block, $\mathrm{c}$, was larger than the length of the wall. This meant that the $M \leq 1 / 6$ NL case from Figure 5-3 was applicable for this loading direction. By performing another force and moment balance, maximum and minimum stresses of 2,406 psf and 562 psf, respectively, were estimated. The proportional limit of the blocks from Section 3.1.8 observed in material testing was approximately $3,000 \mathrm{psf}$ (or $21.3 \mathrm{psi}$ ). It can be seen that the maximum stress was below the proportional limit and this is corroborated by the lack of bulging or visible failures in the blocks post-testing.

In the second half of the cyclic loading, the actuator pushed on top of the wall. Another statics analysis showed that in this case, $\mathrm{c}$ was shown to be less than the length of the wall L. This meant that the M $>1 / 6$ NL case from Figure 5-3 was applicable for the push loading direction. A similar force and moment balance yielded a maximum stress of 2,402 psf and a depth, c, of 7.34 feet. This result was very encouraging in its symmetry with the other direction of loading. This likely means the assumptions made in this model were appropriate.

The same analysis was performed on Walls 2, 3 and 4 with unrealistic results that showed the limits of the assumptions made in this model. This is likely due to the presence of the attached OSB sheathing on these wall specimens. The load path for shear 
transfer is entirely different due to the nails transferring load from the top plate to the shear panel and then through the screws into the straw block walls. Also, even though a $3 / 4$ inch gap was built between the bottom of the OSB panel and the concrete foundation per NDS wood construction standards the panel corners came into contact with the foundation at higher drift levels. The compression damage at the corners of the panels can be seen below in Figure 5-4.

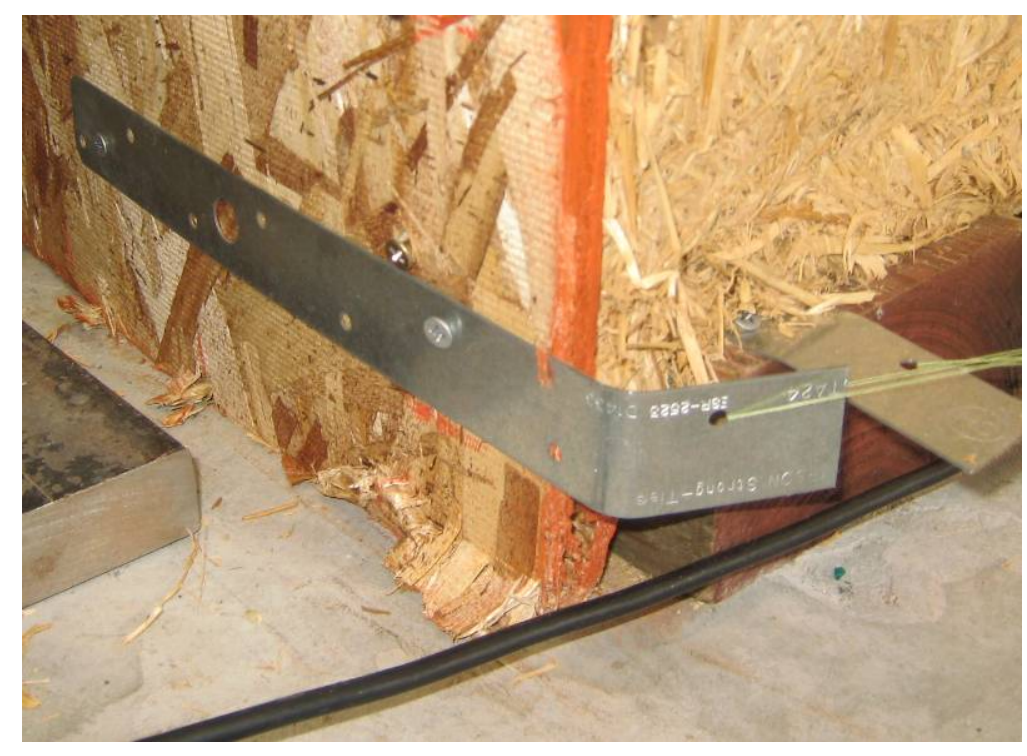

Figure 5-4: Damage at Corners of Shear Panels

Although the corners of the panels were sacrificed, no blocks from any of the sheathed wall tests exhibited damage that indicated stressing past the proportional limit. The same can be said for the condition of the blocks from Wall 1, which is the most lightly detailed situation, relying on the straw blocks alone to transfer loads to the foundation. Neither the straw blocks nor the prestressing bars appeared to yield or fail even at drifts approaching $6.75 \%$. Most structures cannot be expected to withstand these high of drifts, but the wall specimens displayed very encouraging ductility. 


\subsubsection{Forces within Wire Rope versus Thread Bar}

Walls 3 and 4 were detailed exactly the same (OSB sheathing on one side) with the only difference being the method of prestressing (thread rod versus wire rope). The results from these two wall tests are presented in Table 5-2.

Table 5-2: Maximum Forces within Prestressing in Walls 3 and 4

\begin{tabular}{ccc} 
& Wall 3 & Wall 4 \\
\hline \hline LCW0 (kips) & 5.02 & 11.15 \\
LCW1 (kips) & 4.06 & 4.38 \\
LCW2 (kips) & 3.75 & 7.53 \\
LCW3 (kips) & 6.53 & 7.29
\end{tabular}

The maximum tensile force measured in the wire rope loops was $6,530 \mathrm{lbs}$ while the yield point observed in material testing appeared around 6,000 lbs. Despite this, no visible signs of yielding (broken strands) were found upon disassembly of Wall 3. The ultimate strength measured in the wire rope sample was 7,670 lbs and the forces observed during testing did not reach values this high.

Despite the performance differences (higher loads for Wall 4 at similar drifts compared to Wall 3) the fact that the tensile forces were also much lower should be noted. This is likely due to the fact that in the cyclic testing based on displacement the cable loops had lower stiffness. This can be seen clearly in the earlier material comparison (Figure 3-24). Assuming the tops of the prestressing to be fixed to the top plate, at the same drift amounts in each specimen, the tendons would have elongated the same amount. On a basic level, the much less stiff wire rope would see lower forces at 
the same strain. This fact appears to be the main factor in the performance difference between Walls 3 and 4 .

\subsubsection{Thread Bar Yielding and Elongation}

Another interesting finding from the washer load cell data was the asymmetrical loading of the thread bars in the testing of Wall 4. In Walls 1-3, LCW0 and LCW3 recorded the highest tensile values. This makes sense given their location (the boundary or end cells). Yet Wall 4 LCW2 recorded higher loads than LCW3 at the same drifts. This was most likely caused by the very low readings seen in LCW1 and the subsequent redistribution of tensile forces. LCW0 recorded tensile values even greater than were seen in the heavily detailed Wall 2. The forces approached the yield point of the thread bar while the adjacent bar saw forces nearly three times smaller. This especially high tensile force $(11,150 \mathrm{lbs})$ caused considerable elongation. This deformation manifested itself in the tightening nut ending up approximately one half inch above its original tightened location upon the conclusion of testing. The evidence of this elongation is shown in Figure 5-5.

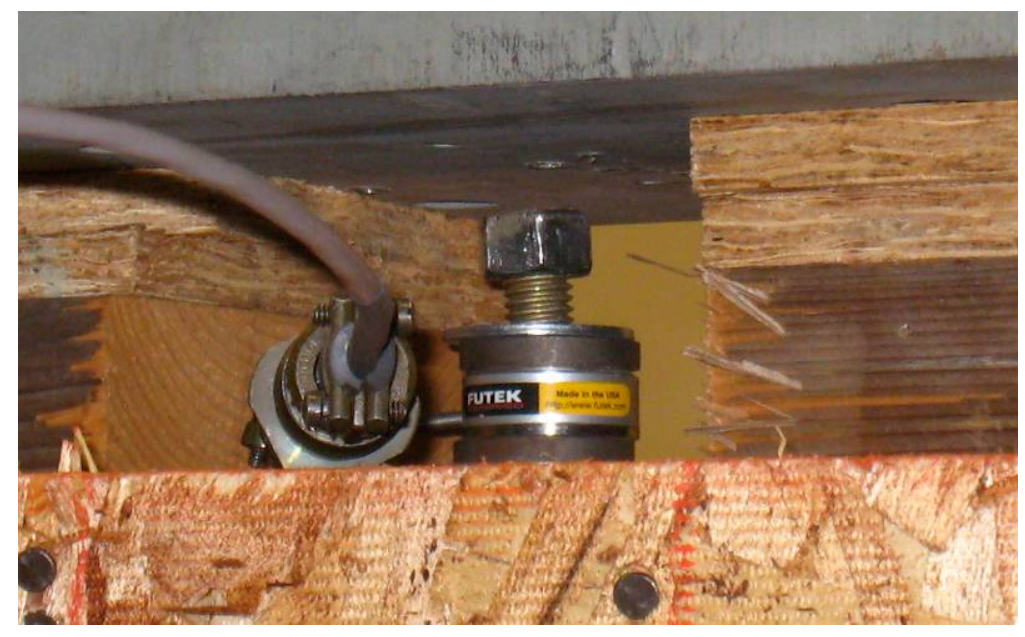

Figure 5-5: Thread Bar Elongation Due to Large Forces 
Upon disassembly of the wall, no major differences were evident between the bar measured by LCW0 and LCW1. This discrepancy was likely caused by some shift or irregular settling of the wall during testing. In the long term creep investigation that is to follow in this chapter, rather large fluctuations can be seen in the measured forces. These fluctuations were caused by a variety of factors, one of which is out of plane movement of the wall specimen. During the testing of Wall 4, the out-of-plane force resisting frame was compromised, and although it was quickly repaired, this shift could explain the low forces in LCW1. For example, a shift could have caused the washers under the tightening nut to move on the top plate. Since the bars are all part of a structural system, a drop in the tensile force in one bar would cause the two adjacent bars to experience larger loads caused by the redistribution of stresses. This redistribution also highlights another advantage in redundancy and ductility of having prestressing bars spaced 2 to 3 feet apart.

\subsection{Wall End Deformation}

While visible yielding or bulging of the straw blocks may not have occurred during wall testing, the walls did noticeably deform as a whole. This global deformation was captured with various instruments and is most clearly seen by looking at the data from the displacement transducers positioned on the ends of Walls 3 and 4. DTR4 was located immediately under the actuator head while DTR5 was placed on the opposite end. So for pull cycles, DTR4 would measure compressive deflection and DTR5 tensile, and opposite for push cycles. The data recorded at the peak deflections of the primary cycles from Walls 3 and 4 is found below in Figures 5-6 and 5-7; in the legends, "(C)" represents compressive deflection readings, and "(T)" tensile. 


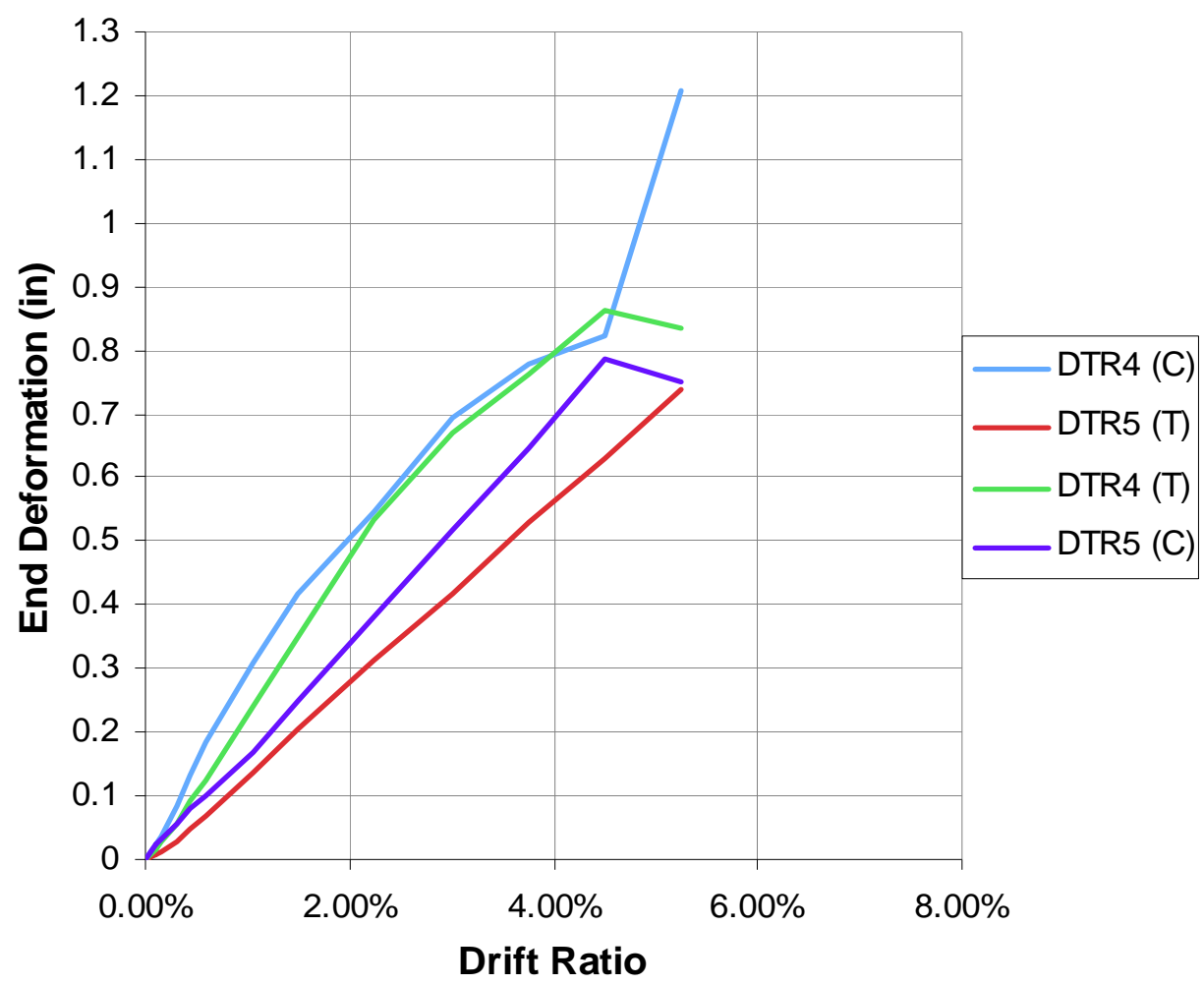

Figure 5-6: Wall 3 End Deformations

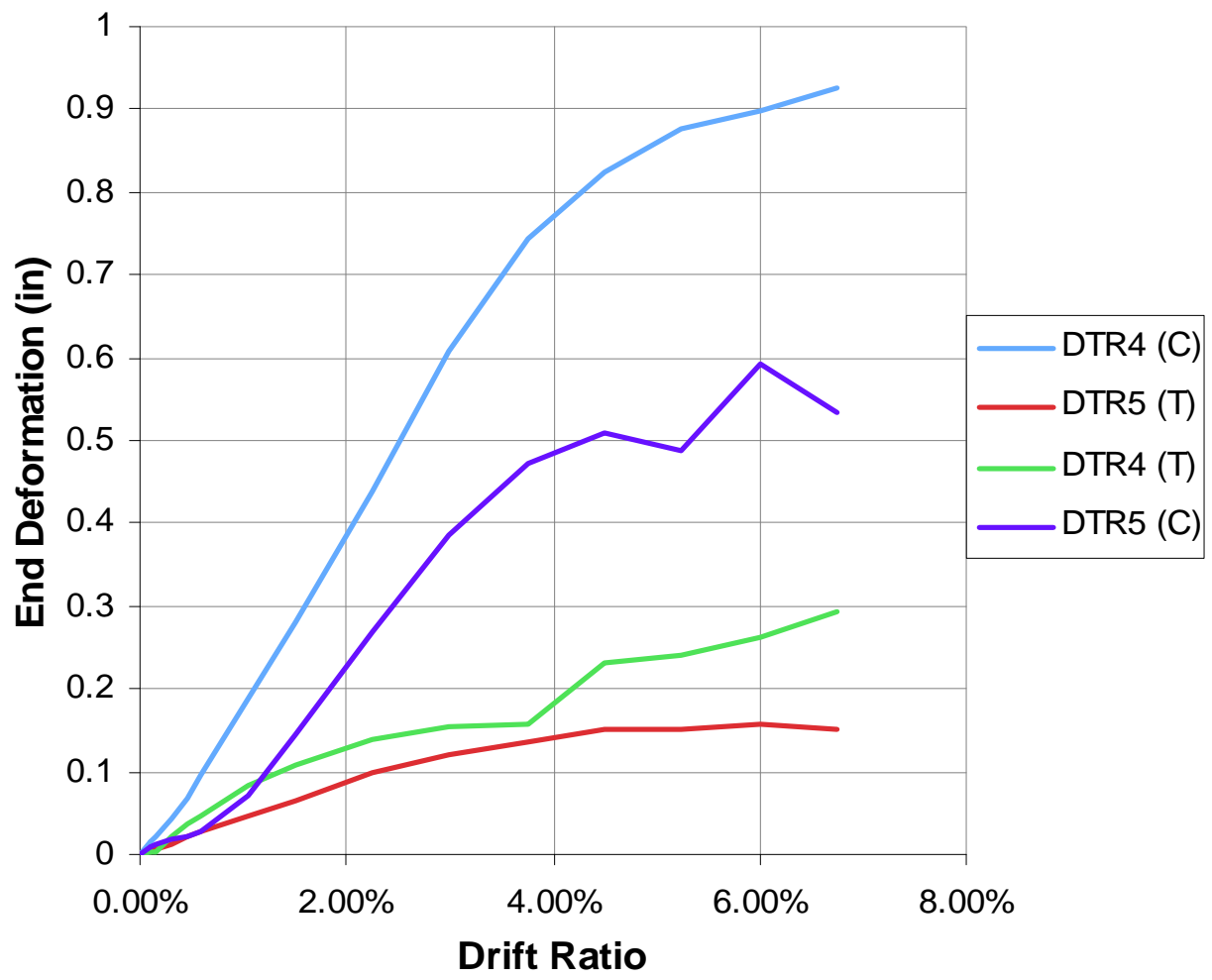

Figure 5-7: Wall 4 End Deformations 
Two main things are obvious from the figures above: first, the effect of the actuator is evident in the increased values for both tension and compression of DTR4 versus DTR5. For example, in Wall 4, at opposite ends of the $6.75 \%$ drift cycle, the end under the actuator was compressing 0.92 inches, while the far end only compressed 0.53 inches. A similar discrepancy can be seen in the tensile values from Wall 3 where at $4.5 \%$ drift under the actuator there was 0.86 inches of deformation while only 0.63 inches at the opposite end of the cycle.

It should be noted that during cyclic testing, the actuator's pull cycle was first. This means DTR4 went into compression while DTR5 went into tension during the first half of the loading cycle. It is possible that the differences between the two deformation readings are because during the first half of the loading cycles, especially at higher drifts, the blocks became permanently compressed.

This is all due to the physical limitations of the testing setup. In real-world applications, the floor or roof sheathing would evenly and uniformly transfer lateral loads to the length of the shear wall; in this case, the actuator and strong wall had to be attached near the end of the wall and the top plate, or drag strut, was intended to distribute the load. Because of this asymmetry and things like the freely pivoting actuator head caused deflections to be larger than normal on that end of the wall.

The second major item to be taken from the plots above is that Wall 3 saw much larger end deformations despite the fact that it held much lower lateral loads than Wall 4. In basic terms, the wire rope was not as effective as the thread bar in holding the blocks together. It has already been discussed that the wire rope was not as rigid, and appeared 
to stretch much easier than the thread bar and the data collected helps to further quantify this.

For example, at $5.25 \%$ drift, the tensile deformation over the full height (i.e. how much the blocks are pulling apart) was an average of 0.20 inches for Wall 4 . At this same drift, and a $32 \%$ lower load, Wall 3 saw an average deformation of 0.79 inches (four times greater). The differences in these numbers can all be attributed to the fact that the wire rope underwent much larger elongations than the thread bar. Because of this, as well as the strength and constructability issues discussed earlier, thread bar is the recommended method of prestressing for this structural system.

\subsection{Shear Interface between Blocks}

Although a full analysis into the movement of individual blocks in relation to each other during testing is beyond the scope of this report, future in-depth research would better explain this phenomenon. This behavior was most clear in the testing of the bare Wall 1. The materials testing into the shear interface strength of the interlocking pegs of the blocks showed that, for a prestressing force near 1,000 plf, a value ranging from 554 to 1,010 plf could be expected in wall testing.

The full scale wall appeared to yield at approximately 1,730 lbs, which would translate to 144 plf. This force was probably when the blocks first started to move very small amounts. The ultimate load where the wall reached its peak load was near 4,690 lbs, which would translate to 586 plf. This value falls within the range of shear interface strengths from earlier testing. 


\subsubsection{Sliding Between Blocks}

Even at the lower drift levels, a grinding or popping noise could constantly be heard as the surfaces of the blocks slid. While this noise is hard to describe in words, it clearly was the straw blocks slipping. At higher drifts, and faster load rates from the CUREE loading protocol, the gaps between the blocks were visibly opening and closing. At the peak drift levels for Wall 1 (6.75\%), a diagonal failure plane clearly formed. This shear failure is very similar to that seen in unreinforced masonry walls along mortar joints and is shown in Figure 5-8.

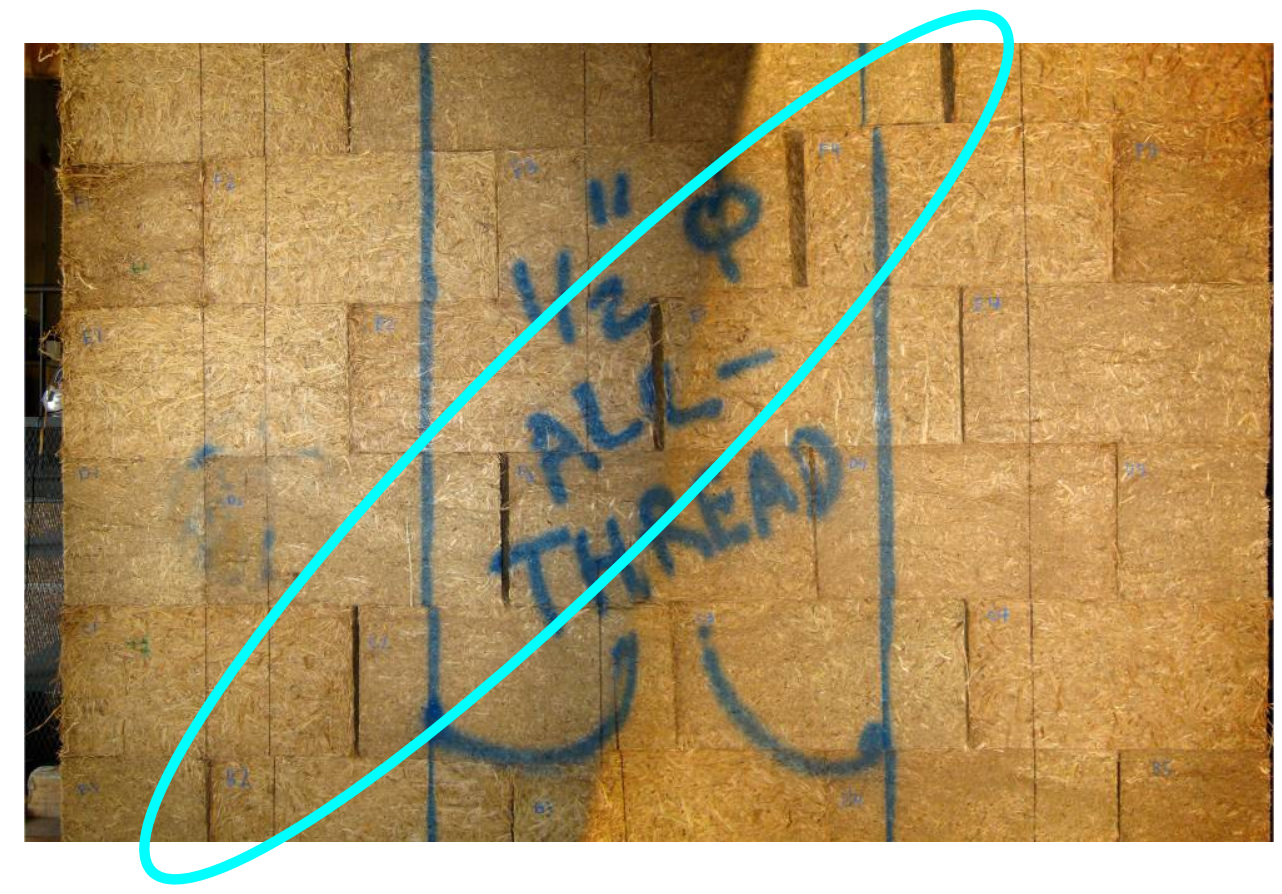

Figure 5-8: $45^{\circ}$ Diagonal Shear Failure of Wall 1

Even though the failure plane "steps" along the weak joints between blocks, it is still a classical shear failure. While some movement did occur at other joints besides the one shown above, these openings closed at the conclusion of each loading cycle. This diagonal failure plane was still obvious after testing was done and the wall was no longer resisting load. Upon disassembly of the wall, blocks along this failure plane had 
noticeable damage to their interlocking pegs. There also were small amounts of loose straw that had been loosened by the friction during testing. Typical damage of a block after shear wall testing is shown in Figure 5-9.

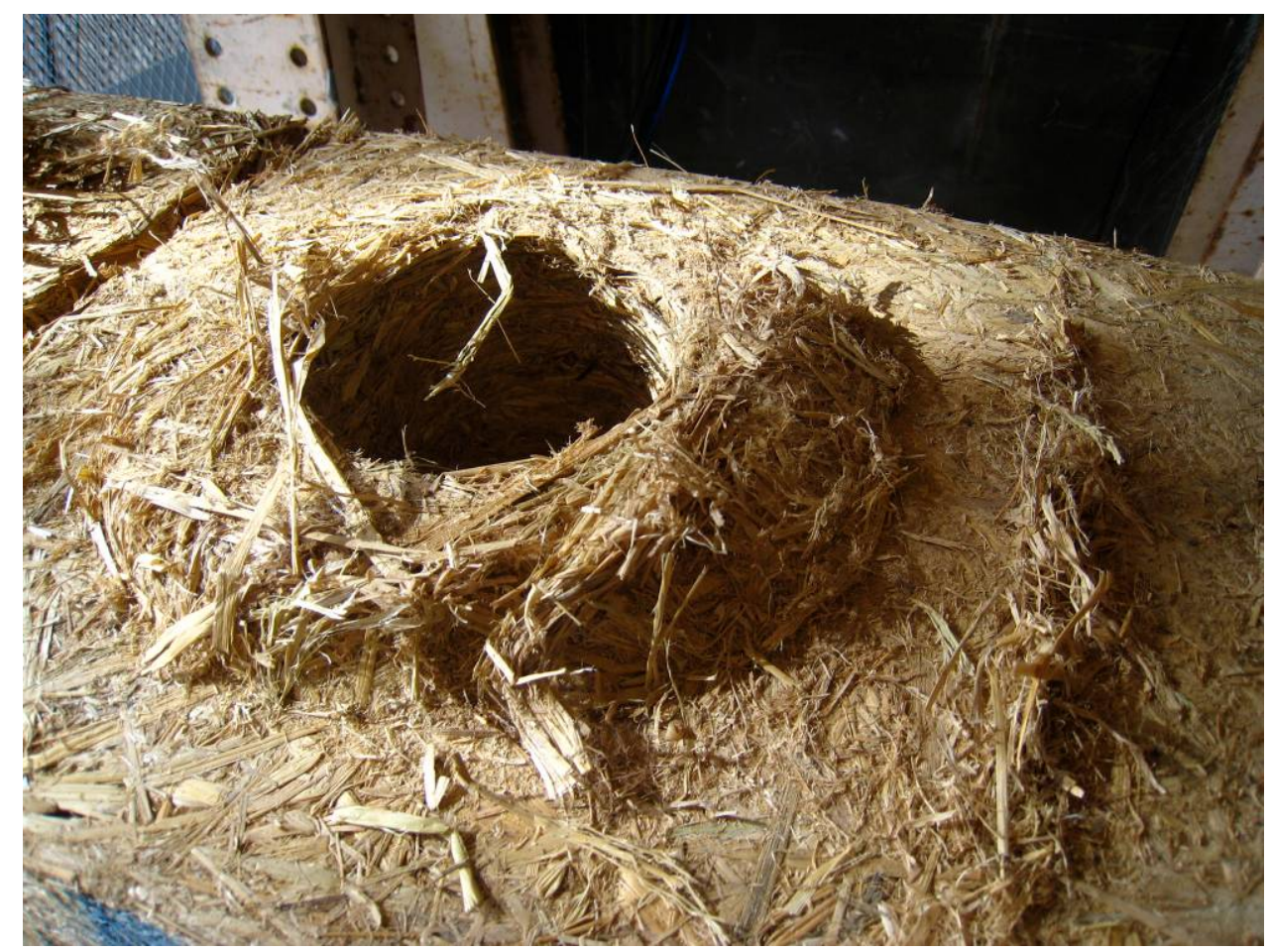

Figure 5-9: Typical Block Damage from Sliding

In the testing of Wall 2 with sheathing on both sides, this inter-block sliding could not be seen and may not have been occurring due to the shear resistance provided by the attached panels. In walls 3 and 4 though, $3 / 4$ inch gaps opened not on a 450 diagonal, but instead vertically where the two panels came together on the opposite side (at the midpoint). This phenomenon can be seen in Figure 5-10. 


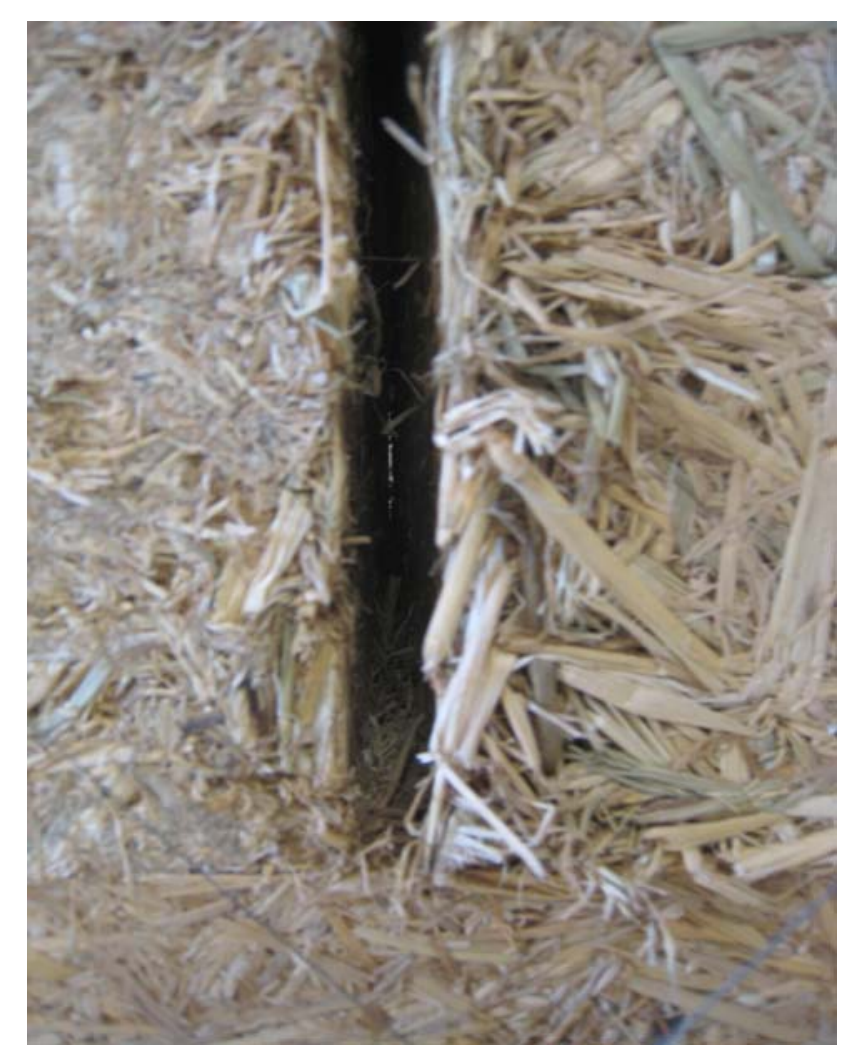

Figure 5-10: Gap Formed Opposite Panel Edges

After the panels were removed, no additional gaps were found, so it can be assumed that the panels held together the straw blocks as a unit to prevent this failure mechanism. One way to avoid this from occurring in future construction would be to place panel edges away from block interfaces. The even dimensions of the wall, panels, and the blocks themselves lead to this detail.

\subsection{Shear Panel Rotation and Deformation}

It has been noted that the OSB panels provided considerable shear resistance to the prestressed straw block systems. With these $1 / 2$ inch OSB panels taking large forces (and even experiencing damage at the corners as discussed earlier), were the panels 
simply rotating or actually undergoing shear deformation? Rotation was clearly seen at higher drift ratios, as shown in Figure 5-11 where the specimen was at 5.25\% drift.

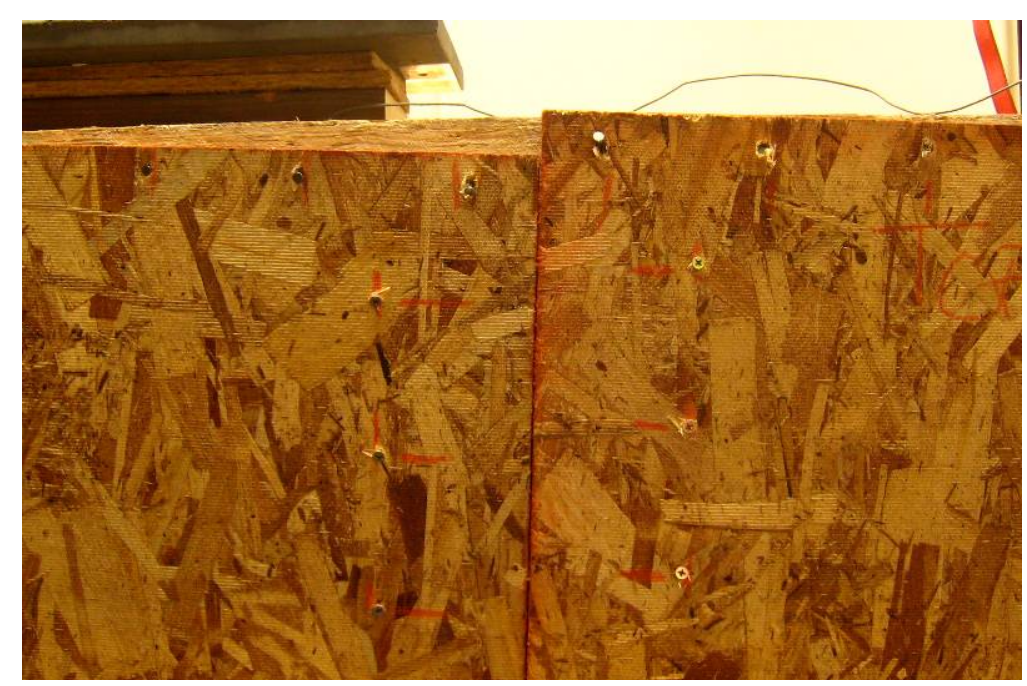

Figure 5-11: OSB Panel Rotation

The rotation shown above at the top plate was also obvious at the foundation and even on the ends of the wall where the panels had been originally applied plumb and level. To investigate this, displacement transducers were placed diagonally and vertically, as shown in Figure 5-12.

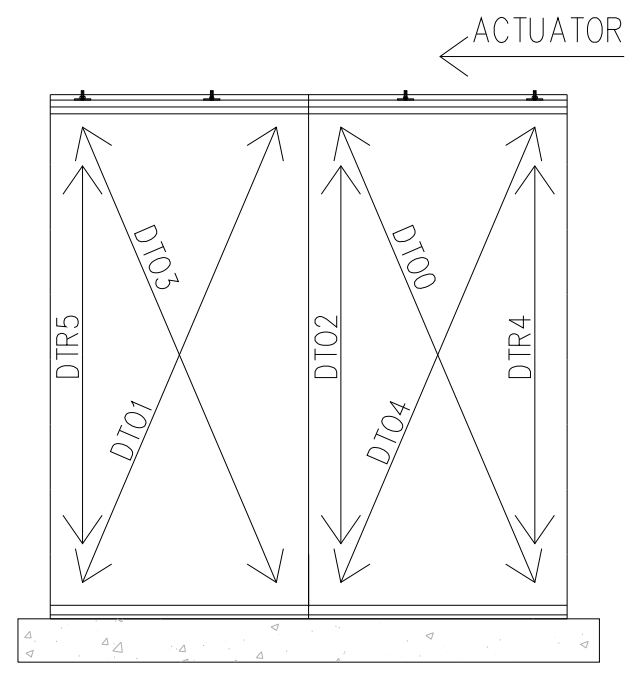

Figure 5-12: Wall 2 Panel Instrumentation 
The maximum deformation data collected from these instruments at primary cycle peaks is shown below in Figure 5-13 and Table 5-3.

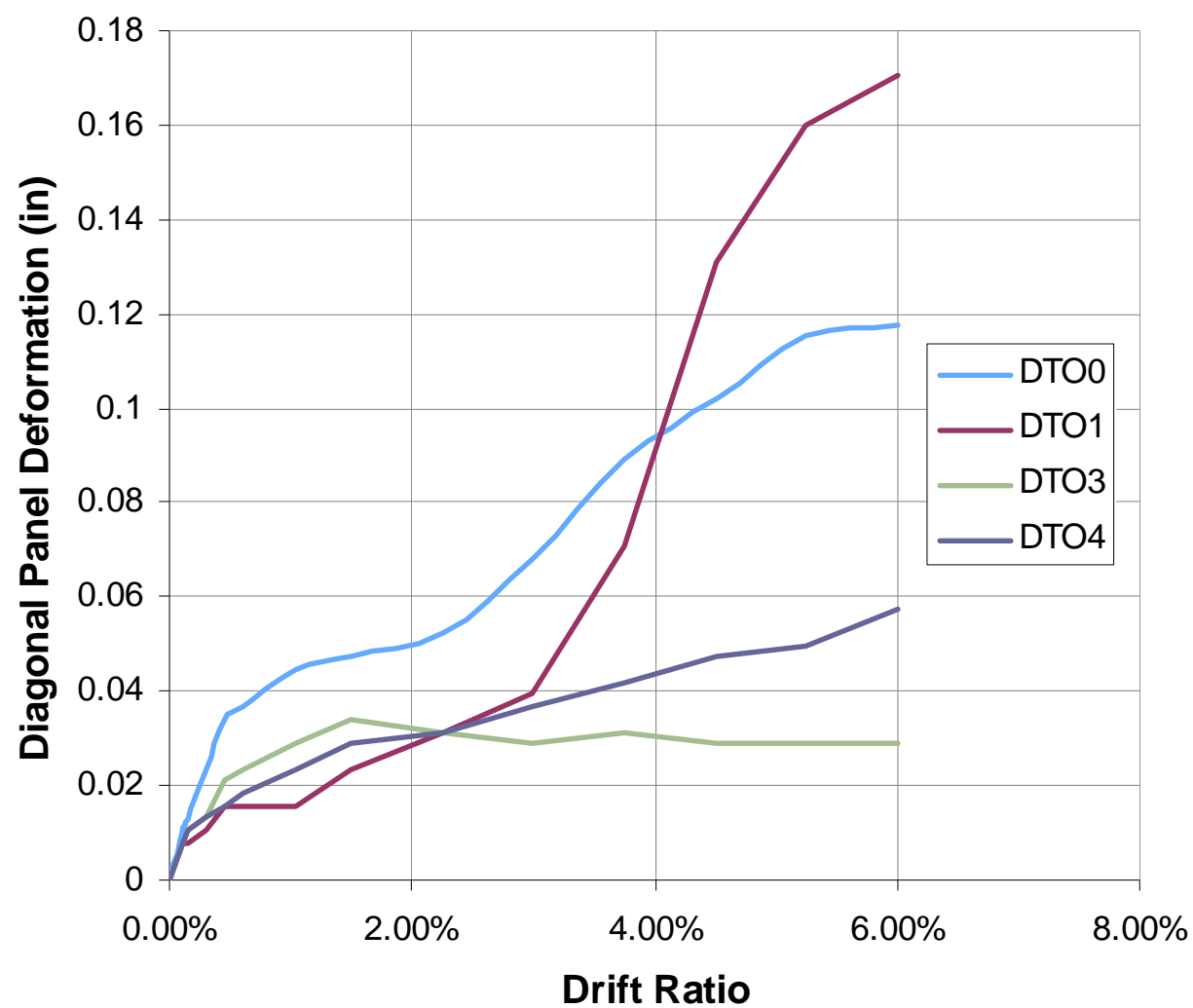

Figure 5-13: Wall 2 Panel Diagonal Deformations

Table 5-3: Wall 2 Panel Vertical Deformations

\begin{tabular}{ccccc}
$\begin{array}{c}\text { DTO2 } \\
\text { (in) }\end{array}$ & $\begin{array}{c}\text { DTR4 } \\
\text { (in) }\end{array}$ & $\begin{array}{c}\Delta_{\mathrm{O} 2-R 4} \\
\text { (in) }\end{array}$ & $\begin{array}{c}\text { DTR5 } \\
\text { (in) }\end{array}$ & $\begin{array}{c}\Delta_{\mathrm{O} 2-R 5} \\
\text { (in) }\end{array}$ \\
\hline \hline 0.039 & 0.055 & 0.016 & 0.053 & 0.018
\end{tabular}

Figure 5-11 shows that the displacement transducers (DTO0 and DTO1) attached near the middle of the double top plate deformed much (4 to 6 times) more than the DT's in the other direction. This is likely caused by a small flexure of the top plate in this location. Although the double top plate is very rigid relative to the rest of the wall, 
prestressing forces were applied to the ends of the top plate and then on each side, 2 feet away from the midpoint. This means there is a relative lack of resisting forces at the top of wall midpoint.

Also, for reasons discussed above, the actuator appears to have had an affect on the panel deformations. The panel immediately under the actuator, DTO0 and DTO4, saw more deflection than the other panel similarly instrumented with DTO1 and DTO3. Despite these differences, even in the worst case at 6\% drift and 12.36 kips lateral load, the panel only deflected approximately 0.17 inches over 107 inches, which comes to $0.1 \%$ strain.

Table 5-3 shows similarly low values for vertical deformations. DTO2, located at the midpoint of the wall, recorded a maximum displacement of only 0.039 inches. Compared to an average of 0.054 inches at each end of the wall, there was a differential displacement of 0.017 inches. This is equal to approximately $0.02 \%$ differential strain over the full height of the wall. Although the panels did deform under very high loads and drift levels, the values were on a small enough orders of magnitude to not cause concern.

\subsection{Shear Transfer along Sill and Top Plates}

Another detailing decision that went through various iterations in the construction and testing process was the transfer of shear from the sill plate to the straw block wall. The two solutions were nails driven through the sill plate sticking up into the straw blocks or Simpson ${ }^{\circledR}$ mending plates with the steel teeth gripping the straw block surface. Displacement transducers on near the bottom of the wall and on the sill plate allowed for an investigation into the effectiveness of this detail. 


\subsubsection{Sill Plate Shear Transfer}

It should be noted that in the case of sheathed walls, the panels transfer a portion of the shear force into the sill plate through their edge nailing. Yet, as will be discussed later, even when the nails fail under large loads at the panel edges, these loads are still transferred from the wall to the foundation through the block-plate interface.

In order to fairly compare the data for the two details, a few items need to be taken into consideration. First, instead of just looking at maximum displacements, displacements will be compared at the peak drift/load, at the design-level $2.5 \%$ drift, and at 4,000 lbs lateral load (chosen as a point well past the yield point, equates to an even 500 plf).

Second, the sill plate was instrumented to investigate whether or not it was correct to assume it remained static with the foundation.

Third, because of physical limitations of the instruments and the methods used to attach to the walls, for Wall 4's data, DTR0 and 1 as well as DTR2 and 3 were approximately 3 inches apart vertically. At the peak drift levels, this translates to a 0.194

inch $((3 " / 96 ") * 6.25 \%=0.194 ")$ theoretical difference. This value was subtracted from the recorded difference in displacement to obtain slip between materials. In most cases the theoretical value was equal or greater than the measured and in this case the slip was assumed to be zero. Table 5-4 gives a summary of the sill plate behavior of Walls 1 and 4. 
Table 5-4: Sill Plate Slips for Various Details

\begin{tabular}{c|ccc|ccccc} 
Detail & \multicolumn{3}{|c|}{ Nails @ 4" } & \multicolumn{5}{c}{ Simpson MP @ 24" } \\
\hline $\begin{array}{c}\text { Point in } \\
\text { Testing }\end{array}$ & $\begin{array}{c}\text { DTR0 } \\
\text { (in) }\end{array}$ & $\begin{array}{c}\Delta_{\text {theo }} \\
\text { (in) }\end{array}$ & $\begin{array}{c}\Delta_{\text {slip }} \\
\text { (in) }\end{array}$ & $\begin{array}{c}\text { DTR0 } \\
\text { (in) }\end{array}$ & $\begin{array}{c}\text { DTR1 } \\
\text { (in) }\end{array}$ & $\begin{array}{c}\Delta_{0-1} \\
\text { (in) }\end{array}$ & $\begin{array}{c}\Delta_{\text {theo }} \\
\text { (in) }\end{array}$ & $\begin{array}{c}\Delta_{\text {slip }} \\
\text { (in) }\end{array}$ \\
\hline \hline Peak Drift & 0.103 & 0.136 & 0 & 0.019 & 0.397 & 0.378 & 0.129 & 0.249 \\
2.5\% Drift & 0.066 & 0.051 & 0.015 & 0.003 & 0.033 & 0.030 & 0.047 & 0 \\
4 kips & 0.076 & 0.085 & 0 & 0.013 & 0.032 & 0.019 & 0.035 & 0
\end{tabular}

The main point that Table 5-4 illustrates is that both details worked and practically zero slip occurred between the sill plate and adjacent blocks. The slip value at peak drift for the Simpson plates was nearly a quarter inch, but it should be noted that this was at $6.75 \%$ drift and 7.69 kips. At this very high drift level, the wall had rotated out of plane in the test apparatus and although it was fixed, this could be the cause of this high displacement. For reference, no slip had occurred at the design-level $2.5 \%$ drift or at nearly half of its ultimate load (4 kips). The minute amount that the nail detail slipped at $2.5 \%$ drift is zero for all intents and purposes since at a higher drift and load (4 kips) no slip was evident.

\subsubsection{Top Plate Shear Transfer}

For all four shear wall specimens, the same top plate detail (nails spaced at 4 inches) was used because of the ease of installation. The top plate to straw block slip data from Wall 4 is found in Table 5-5.

Table 5-5: Top Plate Slip Values

\begin{tabular}{cccccc} 
& $\begin{array}{c}\text { DTR2 } \\
\text { (in) }\end{array}$ & DTR3 & $\Delta_{2-3}$ & $\Delta_{\text {theo }}$ & $\Delta_{\text {slip }}$ \\
(in) & (in) & (in) & (in) \\
\cline { 2 - 6 } Peak Drift & 5.594 & 6.054 & 0.459 & 0.194 & 0.265
\end{tabular}




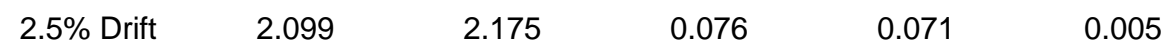

While a value of 0.27 inches of slip for the top plate detail may seem high, it could possibly also be caused by the out-of-plane resistance issue in testing described above. If this is not the cause for this value or the higher value for the sill plate slip, both values are in fact very close (around a quarter inch) which shows that both details work equally as well. The fact that the design-level drift slip value is basically zero is encouraging for this detail. Since results for the two details, at the sill and top plates, are very similar, both work effectively the same at eliminating slip between timber and straw.

\subsubsection{Actuator to Top Plate Connection}

The connection from the top plate to the actuator was done with 6 inch long Simpson ${ }^{\circledR}$ SDS screws and wood blocks to protect the washer load cell instrumentation. A concern was that this gap would create differential movement between the drag strut and actuator. Performing a similar analysis in comparing the top plate movement to that of the cyclic loading ram showed that the average slip (at the points of maximum drift, $2.5 \%$ drift and 4 kips) was 0.041 inches. This proved that the connection was rigid enough to allow only $1 / 24$ of an inch of play at very high loads between the test machine and specimen.

\subsection{Failure of Screws and Nails Attaching Shear Panels}

A phenomenon observed in all shear walls tested with OSB panels was the protrusion of screw heads at the conclusion of the test. This occurred when the panels rotated in a manner discussed earlier and pivoted the screws back and forth in a cyclic manner. Because of the thread of the screws and the relatively soft nature of the blocks 
in comparison with the OSB panels, the screws "wiggled" outward. Figure 5-14 shows two screws that experienced this movement.

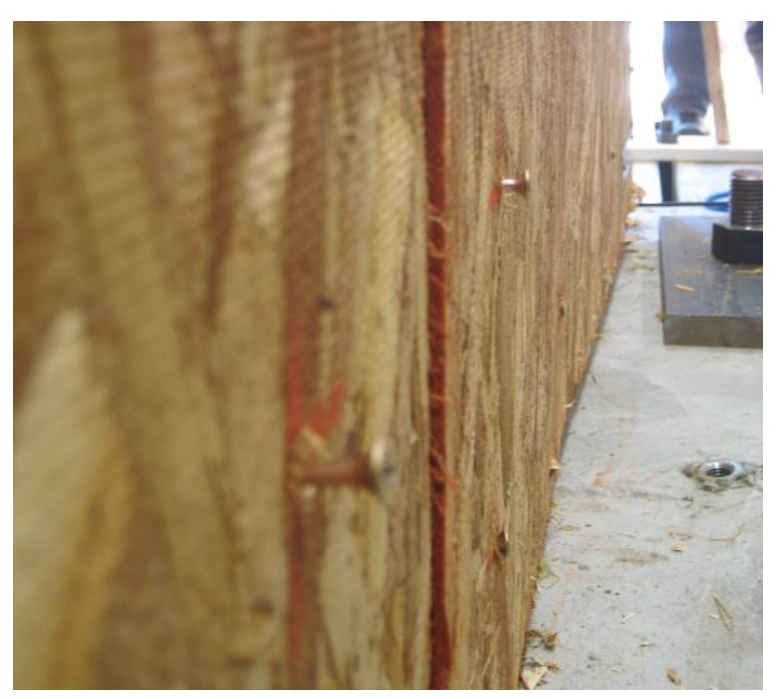

Figure 5-14: Screw Head Protrusion Following Testing

The main reason this occurred was that the screws used in construction were not threaded their entire shank. The top half inch of shaft was smooth and this allowed for the screws to move outward once the straw block backing was damaged as shown in Figure 5-15. 


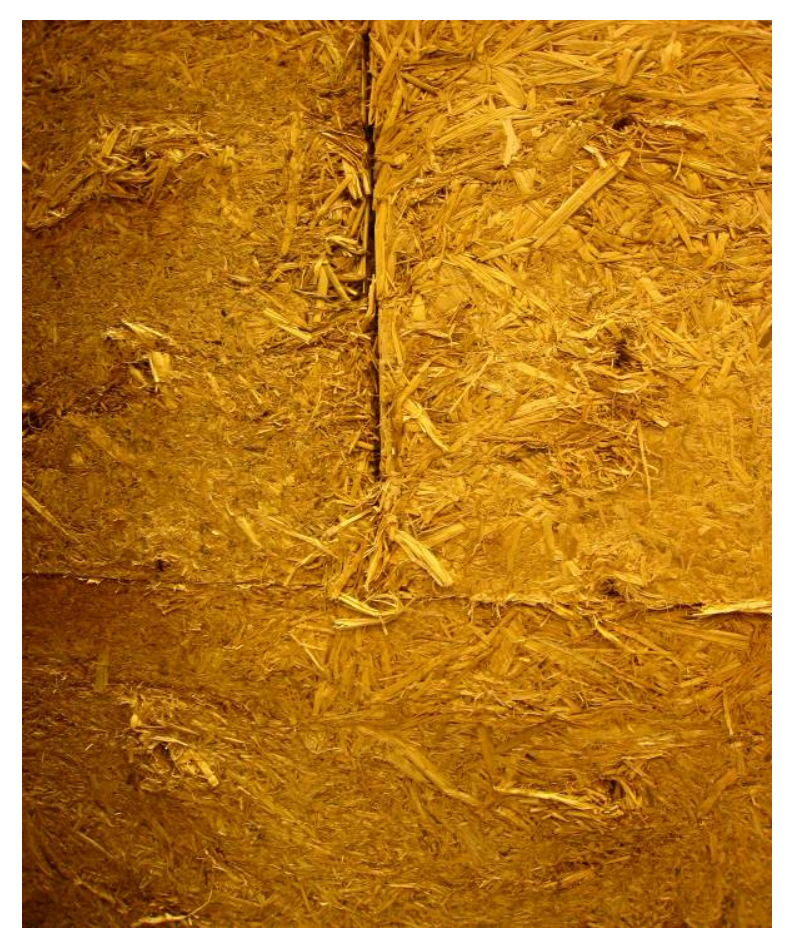

Figure 5-15: Straw Block Damage from Screw Movement

This smooth portion of the shaft also caused issues in driving the screws into the wall. Once the thread had gripped the straw material and pulled in, one had to be careful not to over-drill. Since there was no thread to grip the OSB panel, the screw could spin freely and strip the straw material away. For future construction, a fully threaded screw is suggested to avoid these problems.

Another failure mode, which is very common in typical wood shear wall testing, was the nails at the edges of the panels tearing through the edge. This occurred to some degree in approximately half of the nails attaching the sheathing to the sill plate and is shown in Figure 5-16. 


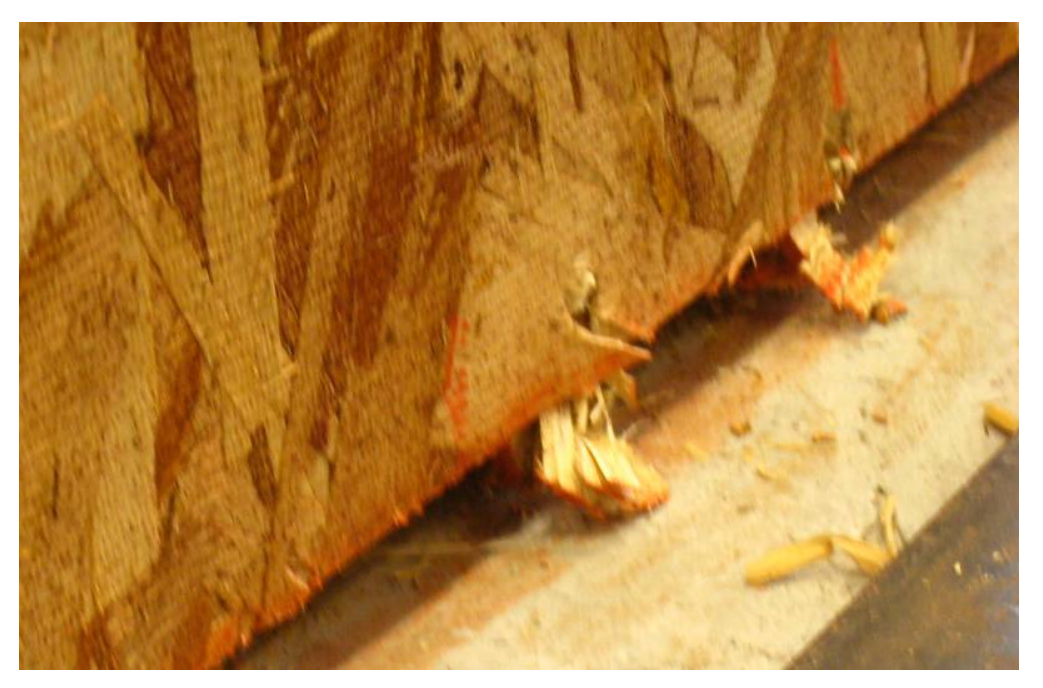

Figure 5-16: Edge Nailing Tear-Through

This failure occurred even though the edge distance requirements were met per

NDS. In fact, wood shear wall strengths are largely controlled by nail strength (including pullout or tearing through edges). Despite the fact that this occurred at drifts as low as $3.75 \%$, all wall specimens withstood increasing loads in subsequent drift cycles. This means that unlike wood shear walls, once this occurs, other methods of shear transfer are utilized to provide superior ductility and strength at higher drift values.

\subsection{Global Relaxation of Shear Wall Specimens}

The earlier investigation into long-term relaxation effects of the straw blocks showed that an individual block loaded to $2,500 \mathrm{lbs}$ could lose as much as 1,000 lbs over a 24 hour period. While a secondary loading following this initial relaxation would reduce the losses, this behavior of the straw material was a concern leading into full scale wall testing. Because of the construction sequence and schedule, the wall would have to be prestressed a day or two before the cyclic shear wall testing would begin. It was 
important to ensure that the intended 1,000 plf prestressing force did not become 500 plf overnight as this would greatly affect the test results.

In order to monitor this process, a data acquisition program was setup to continuously record data from the washer load cells placed on the prestressing rods or ropes. The length of this data record depended on factors such as complexity of wall construction. Figure 5-17 shows the relaxation results prior to the testing of Wall 1.

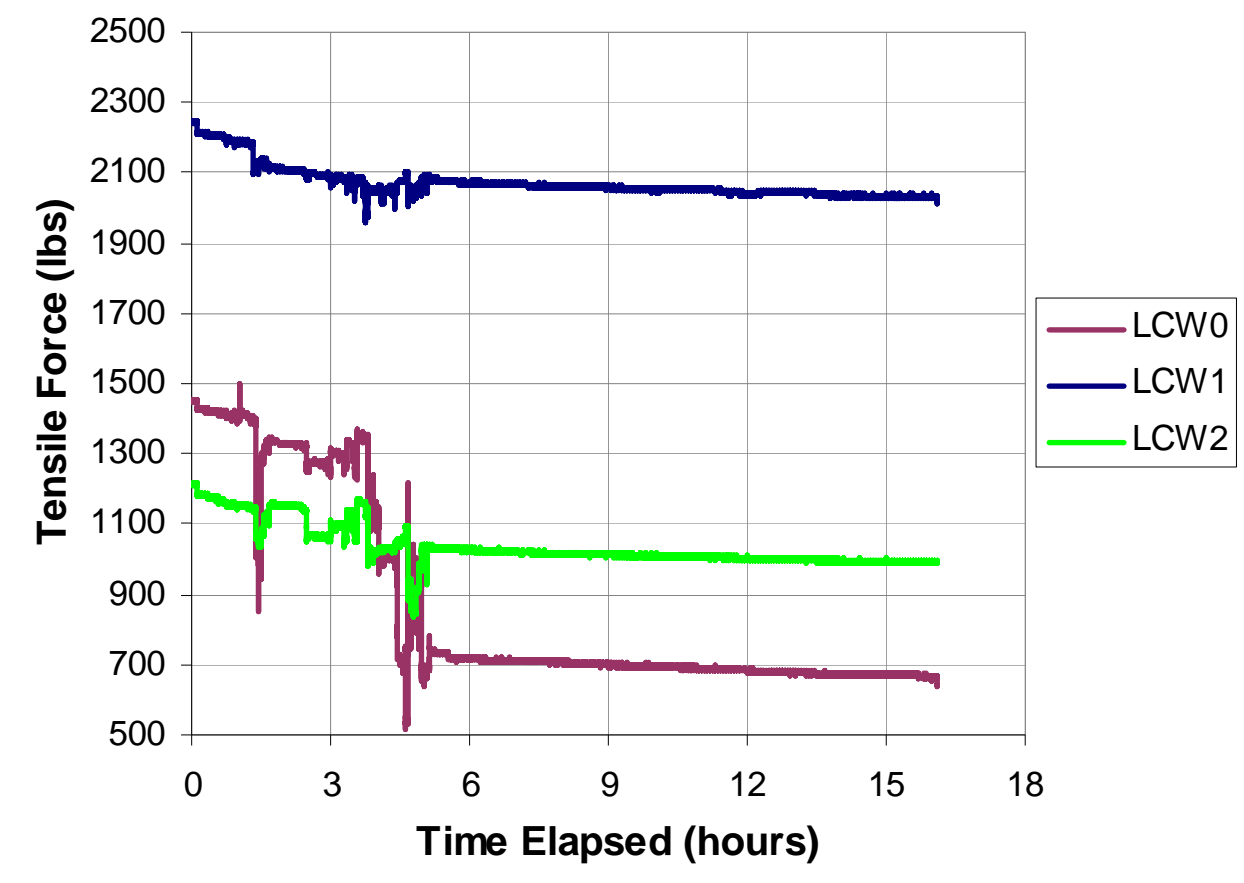

Figure 5-17: Wall 1 Relaxation Prior to Testing

LCW3 had not arrived from the manufacturer in time to be part of Wall 1's instrumentation, so only the three load cells recorded data for the initial test. Data is only presented for 16 hours due to the fact that this wall was unsheathed and could therefore be continuously tightened and adjusted to maintain 1,000 plf of prestressing force. This 16 hour period was immediately before cyclic testing began, and due to the fact that no 
OSB panels were attached to the wall, each nut could be retightened to 2,500 or 1,500 lbs.

LCW1, which started off near 2,300 lbs only dropped to 2,050 lbs and similarly LCW2 dropped less than 200 lbs overnight. The large fluctuations and drop seen in LCW0 appears to be due to out of plane movement and small movements within the wall. As discussed earlier, if the tightening nut and washer were to shift on the top plate, this small change could cause a noticeable change in tensile load. Data from the hours before "hour 0" in the figure above shows many of these fluctuations as the wall was accidentally bumped or shifted in preparation of testing. This first global relaxation test showed the importance of wall stability as well as the need to pretension much higher than the target loads in order to adjust for any losses prior to testing.

Construction of the following shear wall specimen would include attaching four OSB panels with nearly 300 screws and 100 nails before attaching instrumentation and out-of-plane wall support. Because of the time needed to construct the wall, pretensioning occurred two days before testing. With the findings from Wall 1, all thread bars were loaded to 250 to $500 \mathrm{lbs}$ higher than their target values. The data from Wall 2's prestressing can be found in Figure 5-18. 


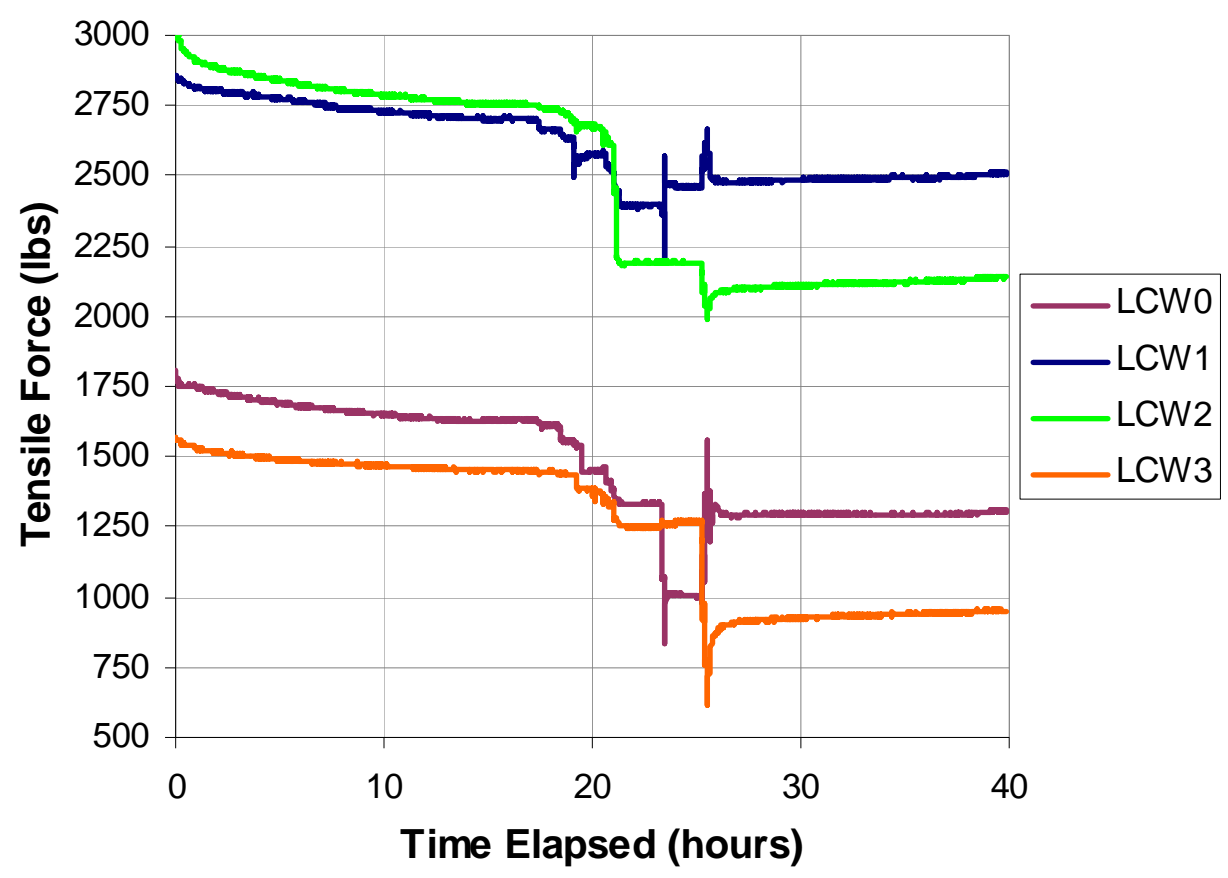

Figure 5-18: Wall 2 Relaxation Prior to Testing

Similar fluctuations to Wall 1 were seen and the losses were actually greater than expected. In the end, the wall ended up being prestressed to approximately 860 plf. While this was not optimal, it did negatively affect the lateral load performance of Wall 2. The large fluctuation seen around 24 hours into the relaxation data coincided with the attachment of the hydraulic actuator ram. The load cells under the actuator (LCW0 and 1) eventually recovered some of the lost pretensioning while the instruments at the other end of the wall showed much greater losses. This is all likely due to the asymmetric loading of the top of the wall.

The move to wire rope prestressing tendons for Wall 3 meant the relaxation behavior observed in the prior two walls might not hold true for this new system. In this case, only one side of the wall was intended to have sheathing attached so only 24 hours passed between prestressing and the beginning of the wall testing. The results for the wire rope Wall 3 relaxation are shown in Figure 5-19. 


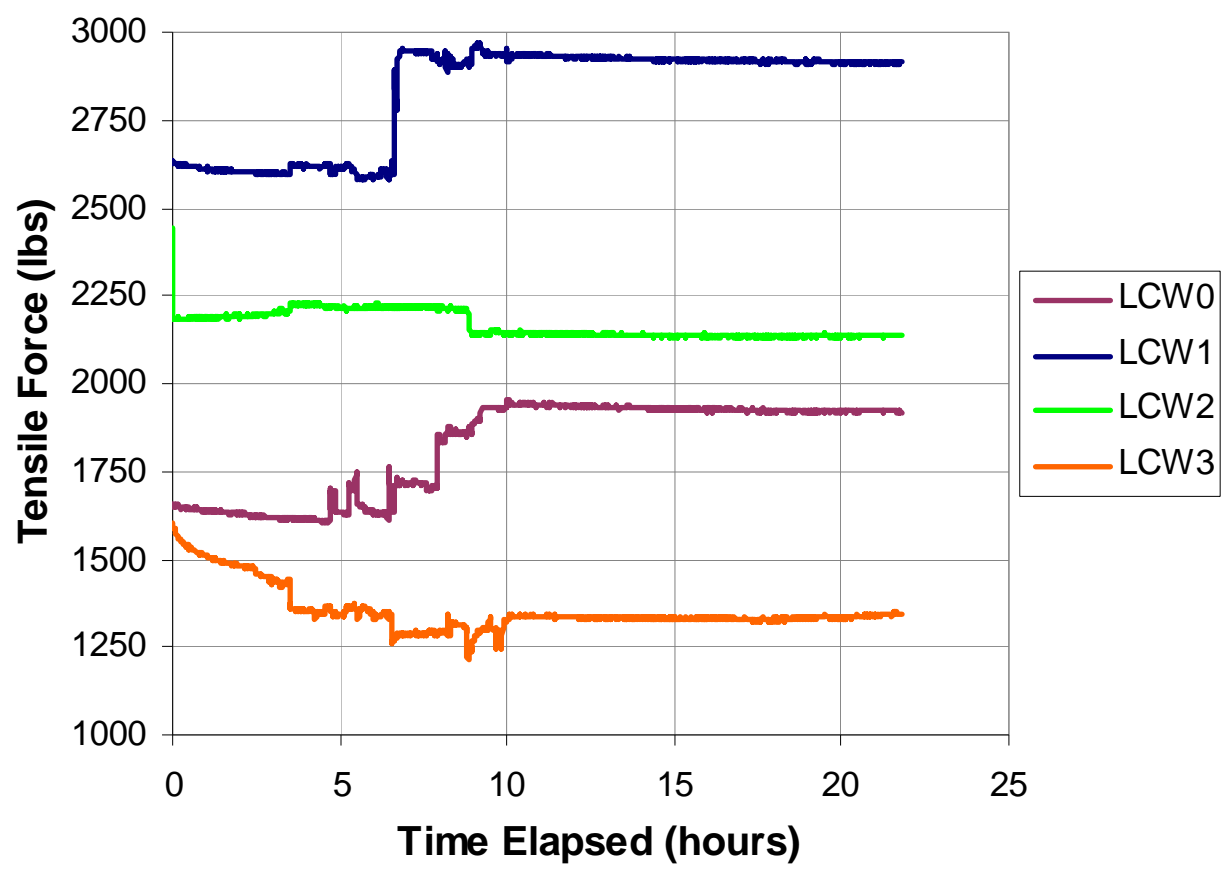

Figure 5-19: Wall 3 Relaxation Prior to Testing

The relaxation results above for Wall 3 were surprising in the fact that the prestressing losses experienced were not as great. LCW2 had an immediate drop near the beginning, likely due to another wall shift, but ended up losing only around $50 \mathrm{lbs}$ over 24 hours. LCW0 and LCW1 appear to have sudden $300 \mathrm{lb}$ increases at around 8 hours time elapsed. This is due to fact that when the actuator was attached, it rested on the tops of the eyebolts sticking up from the top plate. Since a nut was already tightened on these bolts, compressing the load cell, once the actuator's weight was transferred, it registered as an increase in tension for both of these wire rope loops. This problem was fixed by blocking the actuator up higher to be clear of the prestressing bolts prior to shear wall testing. In reality, the tension measured by LCW0 was closer to 2,500 $\mathrm{lbs}$ and in LCW1 was $1,600 \mathrm{lbs}$ which equates to a prestressing load of 950 plf. 
At the time of construction of Wall 4, the method of prestressing to adjust for relaxation loss was refined and an approximately $20 \%$ increase over the target tension was used. One issue with tightening the nuts to pretension was that whenever one location's force was increased, the adjacent prestressing rods see a reduction in force. This, combined with the inherently inexact method of hand-tightening, led to forces being higher or lower than anticipated. The results for the relaxation testing of Wall 4 are found below in Figure 5-20.

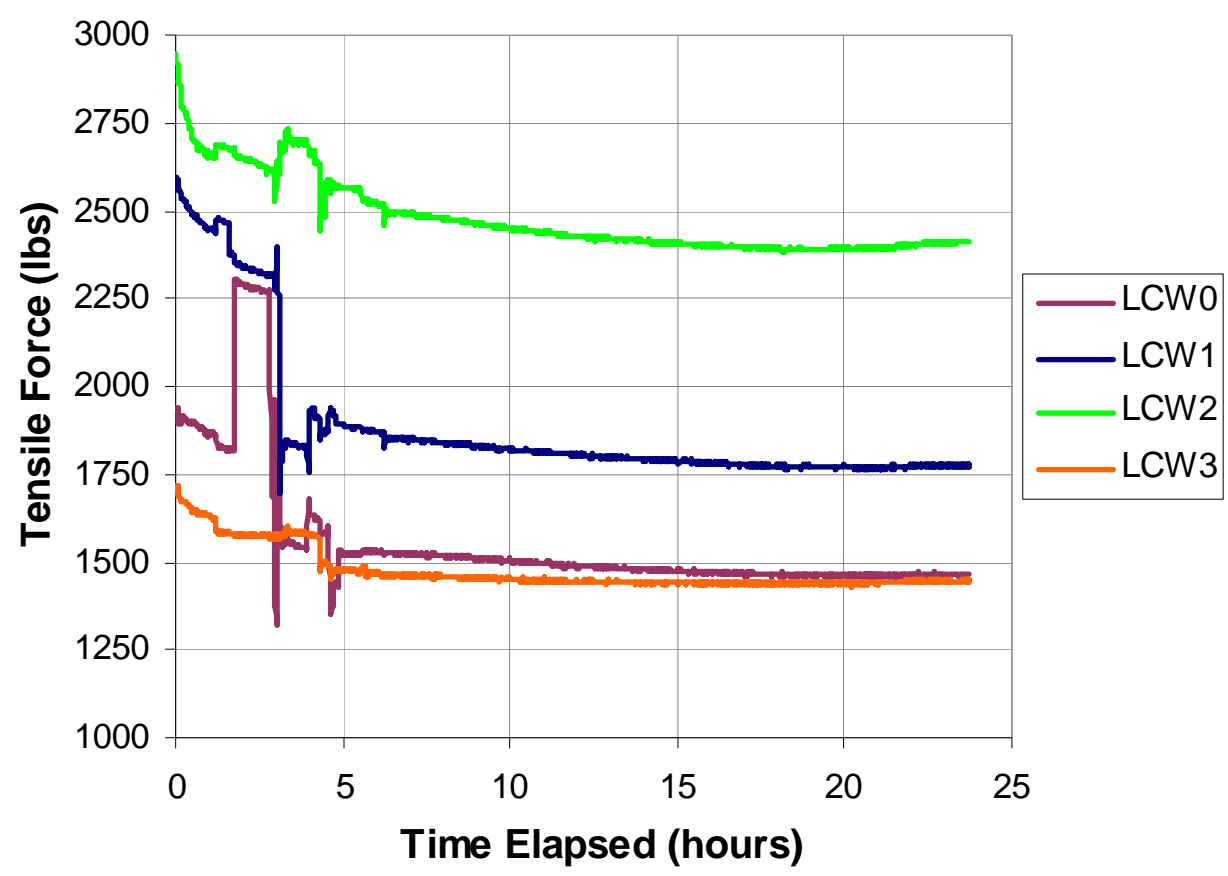

Figure 5-20: Wall 4 Relaxation Prior to Testing

Once again, the attachment of the heavy actuator head is evident 3 hours after data recording began. This is evidenced by LCW0 and 1 again seeing the largest fluctuations in load. In this case, the actuator was not resting on the prestressing members, but the addition of this load is still obvious. Every load except LCW1 were very close to the target values and LCW1 appears to have experienced some sort of shift 
or settling once the actuator was attached. Again, with the OSB panels already attached to the wall, top and sill plates, no adjusting could be done prior to testing. Despite the large loss seen in LCW1, a prestressing force of 886 plf was still intact after global relaxation.

The findings of the global relaxation study were encouraging in that the losses were not as great as seen in the individual block testing. Also, factors such as temperature and humidity did not affect the stresses within the wall as out-of-plane instability and asymmetrical loading of the test apparatus. Both of these issues should be able to be avoided in future construction with the attachment of adjacent floor or roof diaphragms providing uniform support as well as gravity loading. While more research into the long term creep and relaxation behavior of the straw block material is encouraged, the basic findings of this study show that significant decrease in prestressing should be expected and designed for.

\subsection{Development of Backbone Curves}

In cyclic testing of shear wall specimens a force-displacement hysteresis curve is typically produced. These hysteretic curves were presented in Chapter 4 and simply represent the force-displacement behavior of a wall tested using a cyclic loading protocol, in this case CUREE-Caltech. The most effective way of simplifying this data, yet capturing the full wall behavior, is with a backbone curve. This backbone curve is in effect a pushover curve that can be used in inelastic, nonlinear analyses. 


\subsubsection{Prior Investigations into Backbone Curve Construction}

Originally FEMA 356 (American Society of Civil Engineers, 2000) defined the backbone curve as a line drawn "through the intersection of the first cycle for the $\mathrm{i}^{\text {th }}$ deformation step and second cycle at the $(\mathrm{i}-1)^{\text {th }}$ deformation step." This process had built in conservatism and has been proven to exaggerate the rate of strength degradation by many studies such as Dodge (2008) and most notably Elwood et al. (2007). This exaggerated rate could be dangerous in that it can result in an over-estimation of deformations from design-level earthquake demands when using analysis procedures such as FEMA 440 (Applied Technology Council, 2005).

The current accepted standard is ASCE/SEI 41 Supplement 1 (American Society of Civil Engineers, 2007). The Supplement set forth to modify and update provisions specifically for reinforced concrete structures and components, but the recommendations will be used in this study to derive backbone curves. It states "A smooth 'backbone' curve shall be drawn through each point of peak displacement during the first cycle of each increment of loading (or deformation)." Using the CUREE-Caltech loading protocol, this means a point of the curve will fall at the peak displacement point of each primary loading cycle.

\subsubsection{Backbone Curve Generation for Straw Block Specimens}

Using the method described in ASCE/SEI 41 Supplement 1, Figures 5-21 through 5-24 below show each wall's respective backbone curves as constructed from the hysteresis curves. 


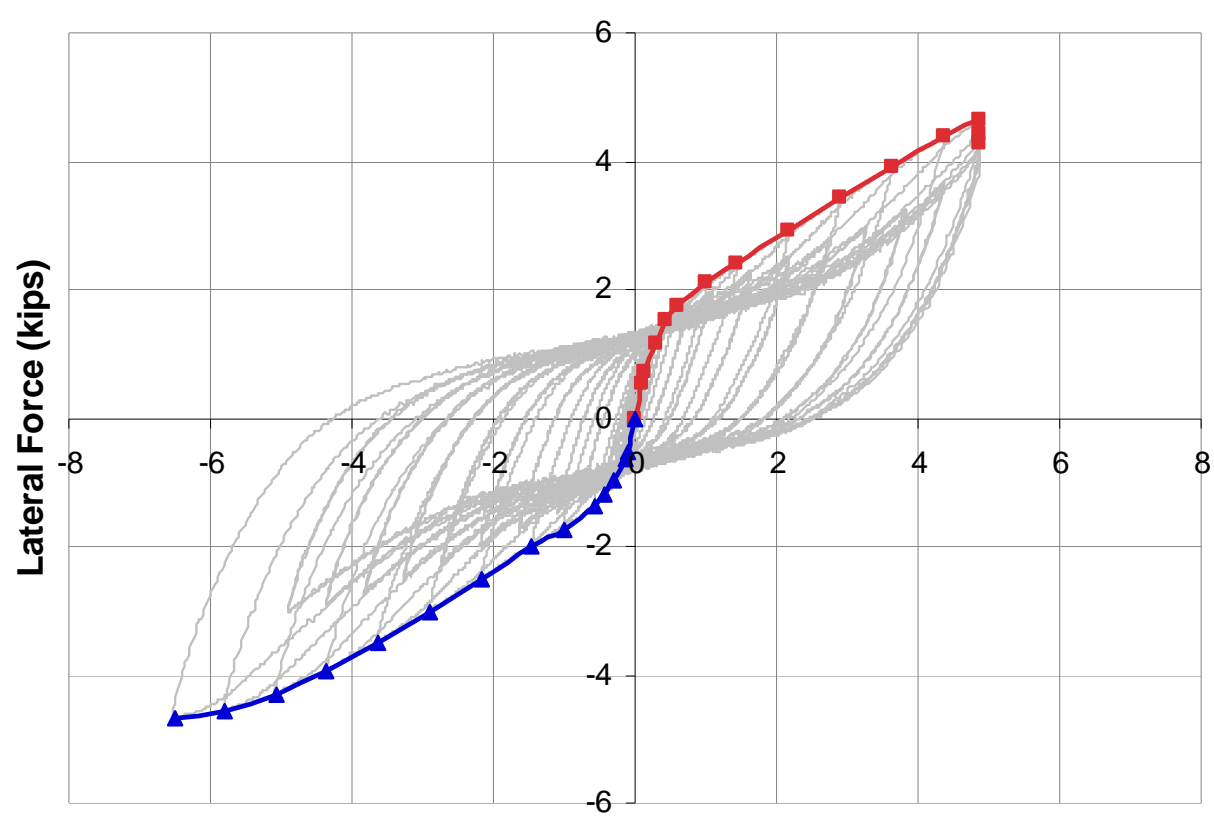

Top Displacement (in)

Figure 5-21: Wall 1 Backbone Curve Construction

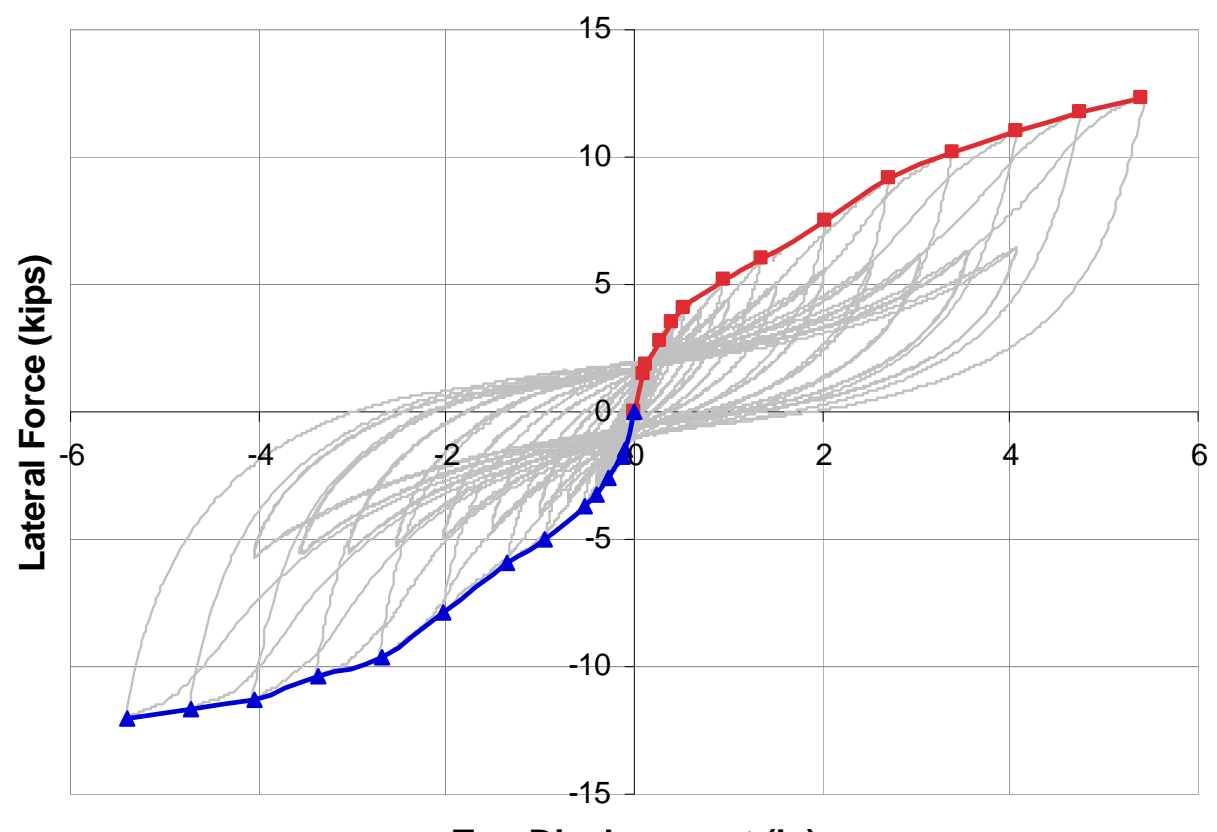

Top Displacement (in)

Figure 5-22: Wall 2 Backbone Curve Construction 


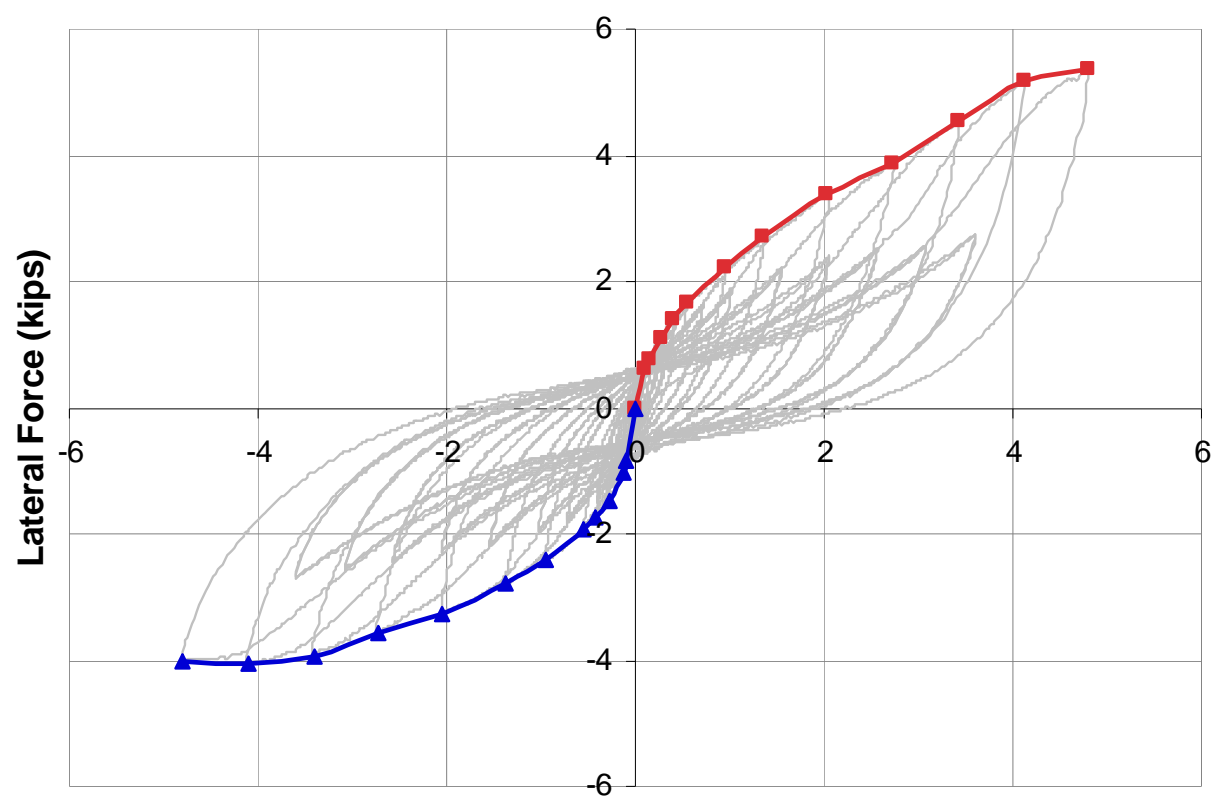

Top Displacement (in)

Figure 5-23: Wall 3 Backbone Curve Construction

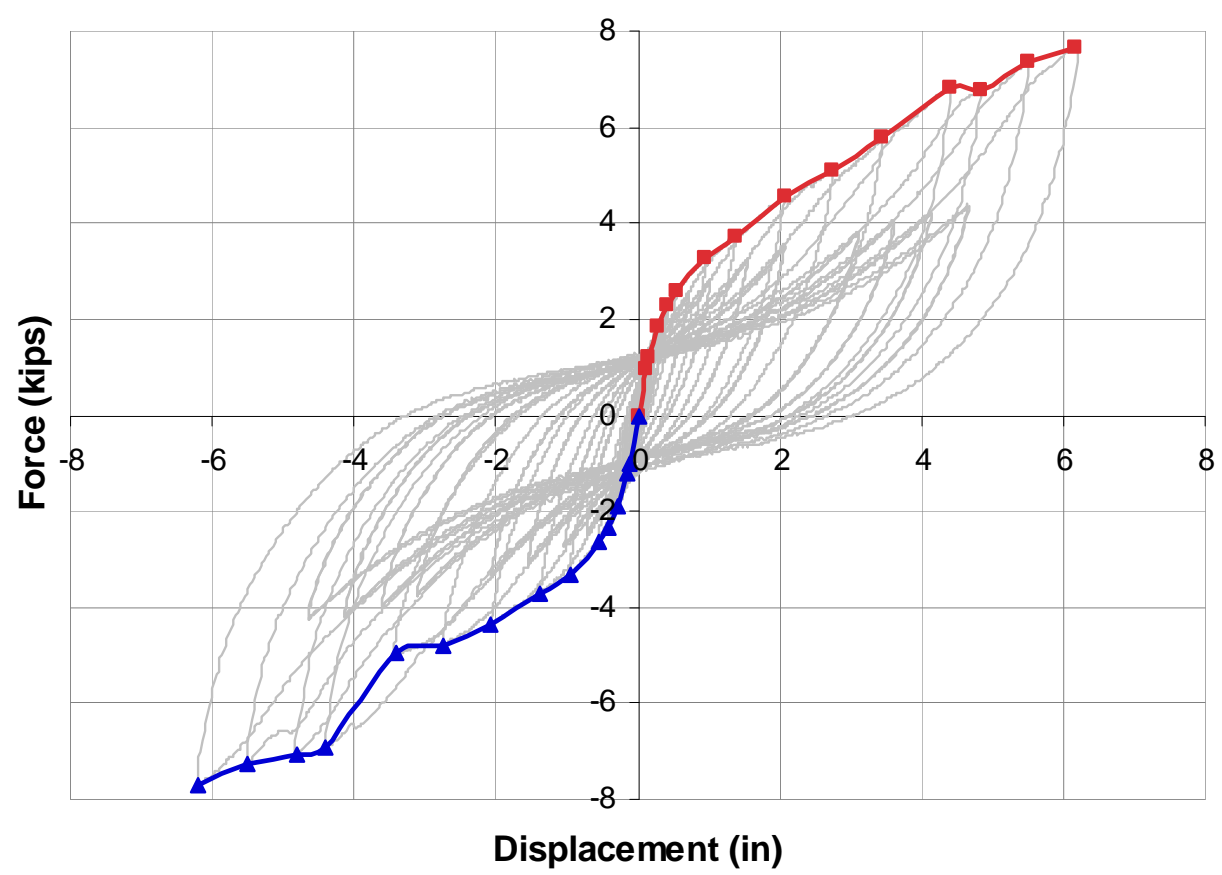

Figure 5-24: Wall 4 Backbone Curve Construction 
An important factor relating to the ductility of the shear walls is that none of the specimens experienced failure or collapse at high drift levels. The backbone curve shows the approximate yield point of the wall, which typically manifested itself as inter-block sliding, and the post-yield branch, which although considerably less stiff, never showed impending failure. The ability to withstand increasing load and deflection after yield has occurred is a very positive trait of a structural system in seismic design. For comparison purposes, all four walls' positive and negative backbone curves are plotted below in Figures 5-25 and 5-26.

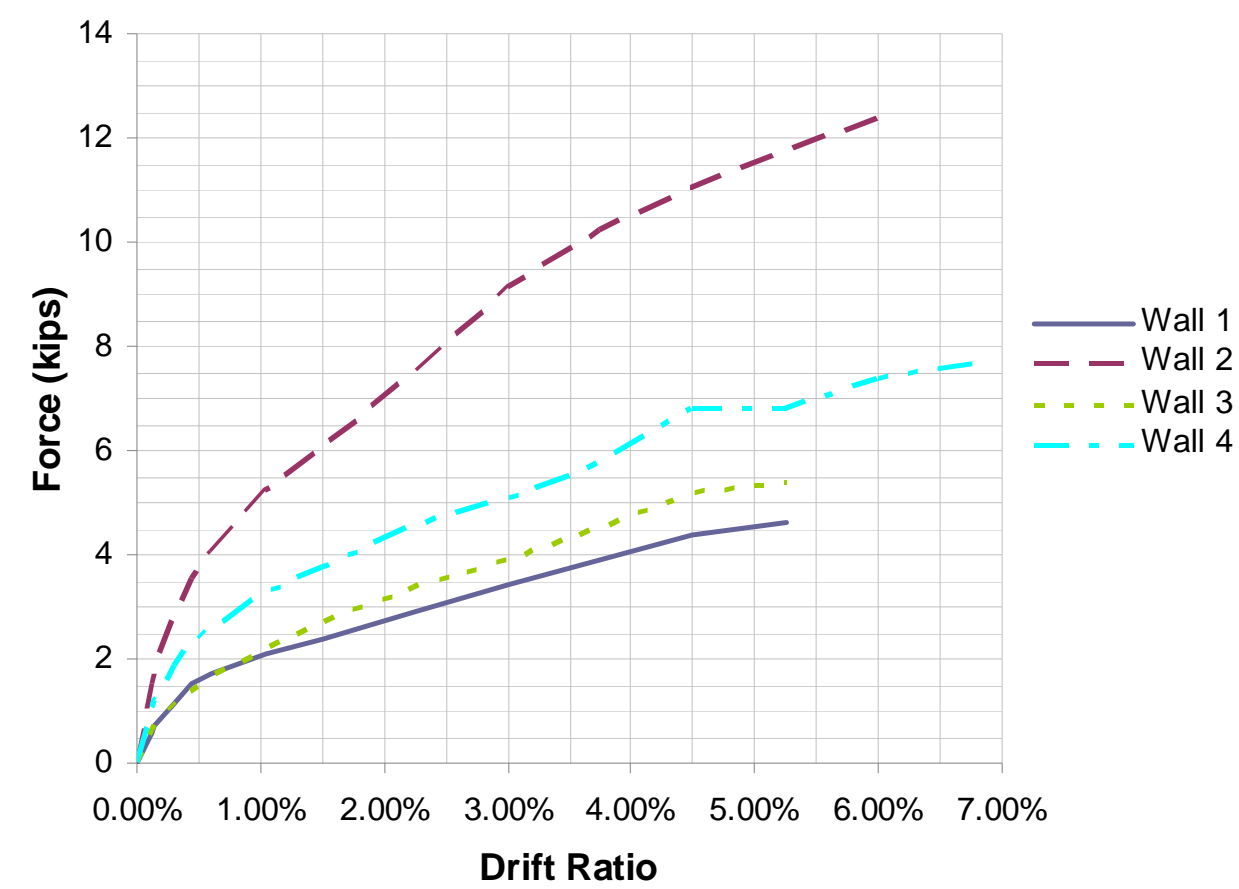

Figure 5-25: All Four Walls' Positive Backbones 


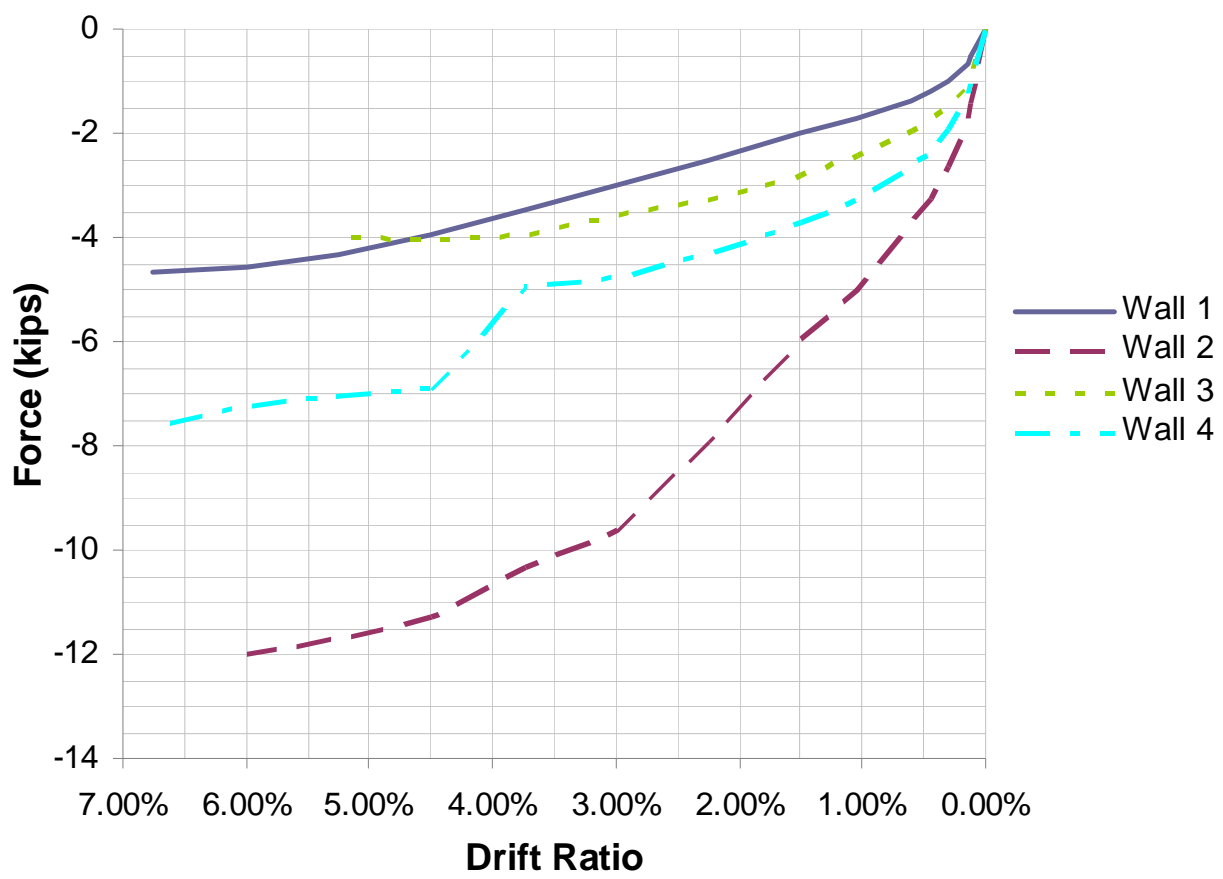

Figure 5-26: All Four Walls' Negative Backbones

As mentioned before, the behavior and strength of the wire rope prestressed Wall 3 is more similar to the bare Wall 1 than to the similarly detailed Wall 4 . It was encouraging to see the backbone for Wall 4, with sheathing on one side, fall nearly halfway between Wall 1 and 2 as anticipated.

\subsubsection{Backbone Comparison to Wood Framed Walls}

A goal in using the CUREE-Caltech loading protocol was to be able to easily compare similarly tested and constructed timber framed shear walls. As this is a widely accepted cyclic loading protocol, various research results were available to compare to. Figure 5-27 below compares two of the straw block wall specimens (Walls 2 and 4) to an assortment of wood shear wall backbones. Dodge (2008) investigated the effects of spray foam insulation on wood shear wall performance. Gatto and Uang (2003) investigated 
the effects of different loading protocol on wood shear wall behavior. Heredia (2010) explored the performance of wood shear walls treated with drywall and stucco. All walls were tested using the CUREE-Caltech protocol, and both Dodge and Heredia tested their wall specimens in the same facilities using the same equipment as the walls in this report. Table 5-6 summarizes the construction detailing of the walls being compared.

Table 5-6: Prior Shear Wall Testing Descriptions

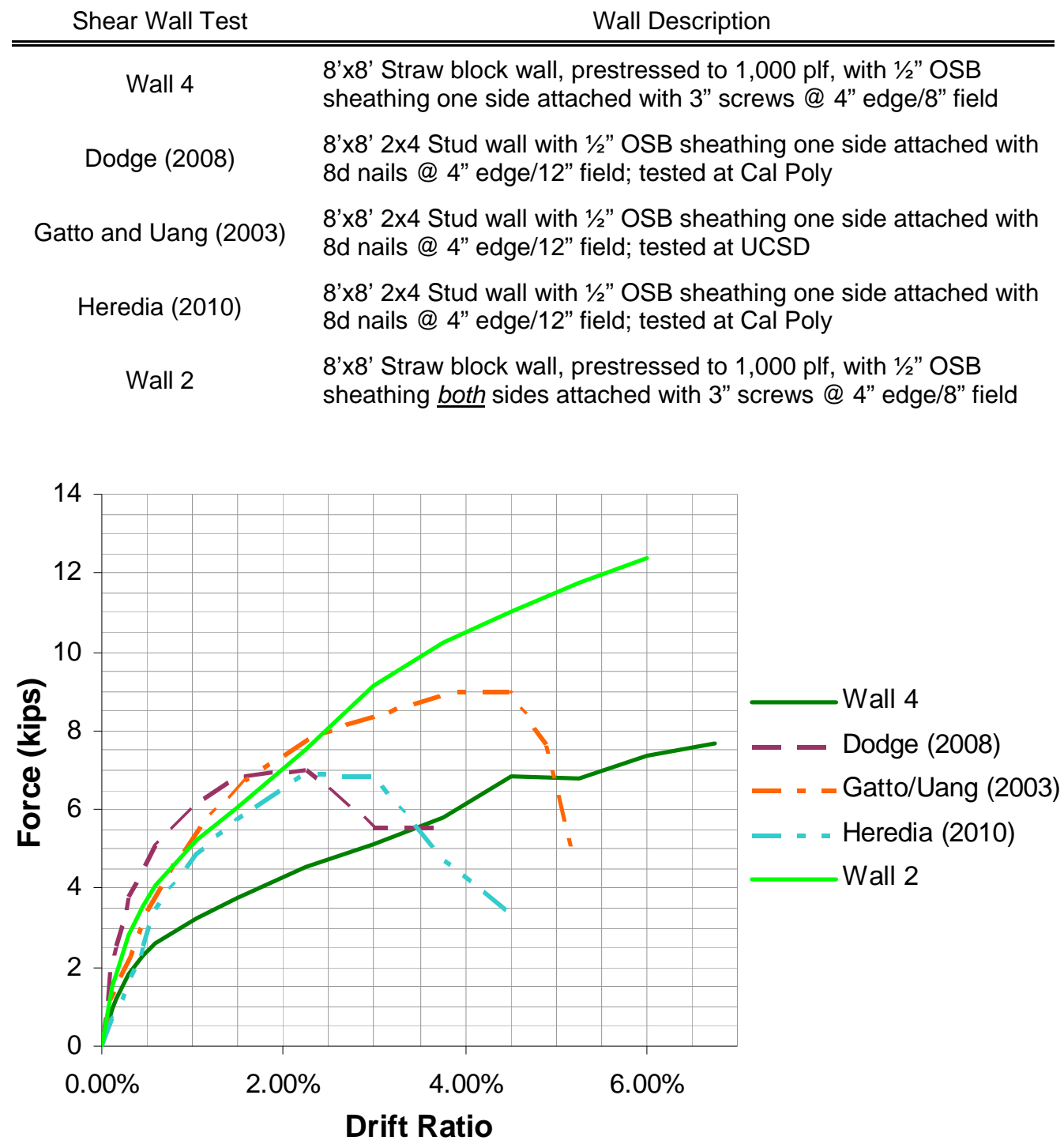

Figure 5-27: Prior Shear Wall Testing Backbone Comparison 
The straw block walls' performance compares to similarly detailed (panel thickness and screw/nail spacing) wood framed walls. Prior to testing and construction, even the heavily detailed Wall 2 was expected to not be as stiff as a conventional wood framed wall. The testing shows that for drifts up to $2.5 \%$, the double sided sheathed wall performed as well or better than wood framed walls. The single sided sheathing Wall 4 showed a similar stiffness for low drifts (up to $0.5 \%$ ) and then its post-yield region is not as stiff as the wood framed walls.

At the $2.5 \%$ drift level, Wall 4 's strength was approximately $65 \%$ of the average of the three wood stud walls presented. It is what happens after this drift level that shows the straw block walls' advantages: both Dodge and Heredia saw walls that started to fail or collapse immediately following this drift level. Both straw block walls show continually increasing loads, even as higher drift levels are approached.

ASCE/SEI 41 Supplement 1 defines the point at which the backbone curve reaches a peak and begins a rapid downward slope as the point of "collapse". The same provision defines the point of $75 \%$ of the collapse deflection as "life safety". Where the straw block walls' ductility and post-yield strength is very promising is that none of the wall specimens appeared to have reached this "collapse" point. This collapse point is typically reached in wood shear walls between 2 and 4\% drift (Dodge, 2008 and Heredia, 2010 ) and at values as low as $0.75 \%$ in much stiffer reinforced concrete walls (Ibarra et al., 2005). While the next chapter will deal with the effects of the decreased stiffness of straw block walls in seismic response, comparing the testing results to conventional lightframed shear walls shows that straw block is a viable lateral force resisting and gravity load bearing building system. 


\subsection{Hysteretic Dissipation of Energy}

The Normalized Hysteretic Energy (NHE) is an important parameter to look at in a shear wall testing program to determine how effective a wall system is at dissipating energy. In the case of the straw block walls, this energy is dissipated by the sliding friction between blocks and by the deformation or rotation of shear panels and subsequent movement of attached screws and nails. The hysteretic energy dissipated is defined as the area under the force-displacement hysteretic curves from testing. This area was calculated using a trapezoidal approximation that is used to evaluate definite integrals. The NHE was defined by Ibarra et al. (2005) as $\frac{\text { hysteretic energy dissipated }}{F_{y} \delta_{y}}$ with $F_{\mathrm{y}}$ and $\delta_{\mathrm{y}}$ being the observed yield force and displacement respectively. These values are quantified later in Section 6.2.2. The hysteretic energy dissipated is a summation of the dissipated energy in all previous excursions in that direction. Figure 528 shows an example of this energy calculation and Figures 5-29 through 5-32 show the Hysteretic Energy Dissipated per each cycle of displacement. For reference purposes, on the plots below, the IBC drift limit of $2.5 \%$ occurs around 40 excursions on the horizontal axis. 


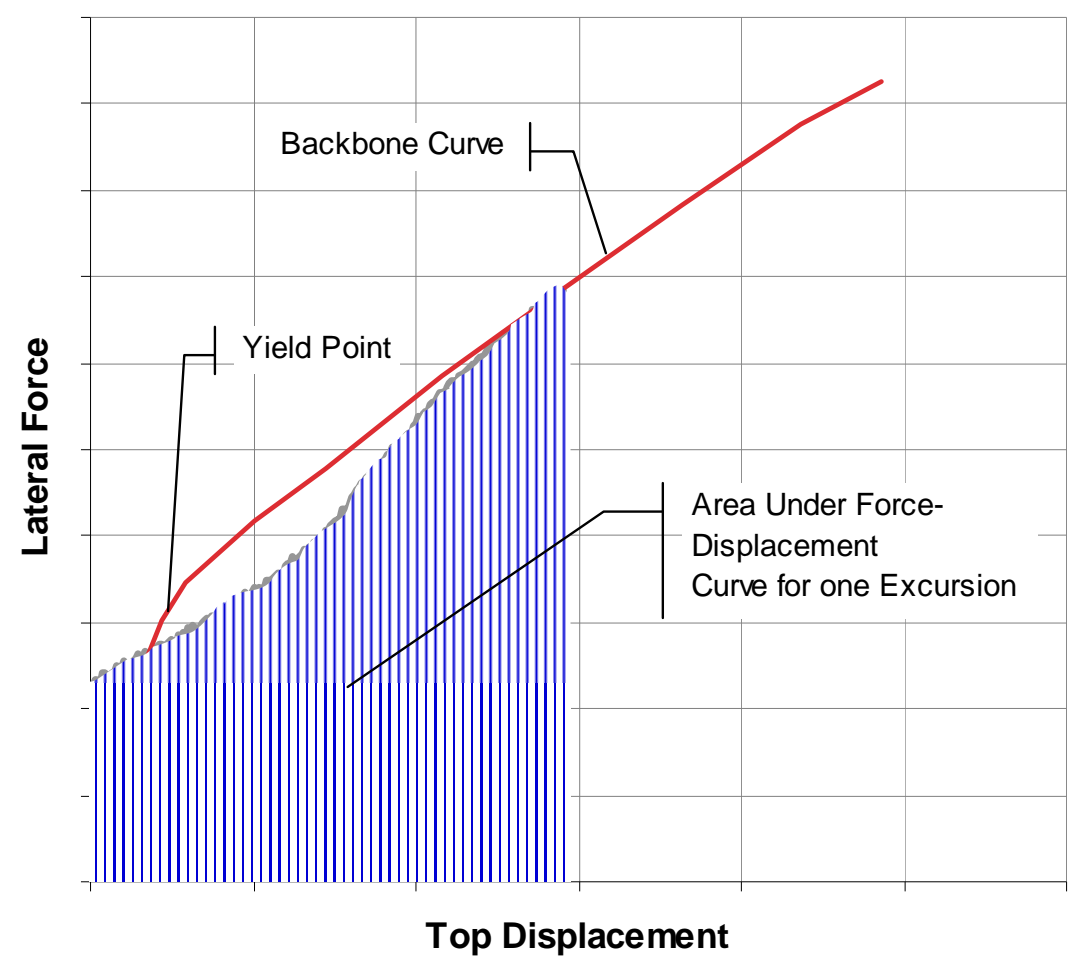

Figure 5-28: Example Hysteretic Energy Calculation

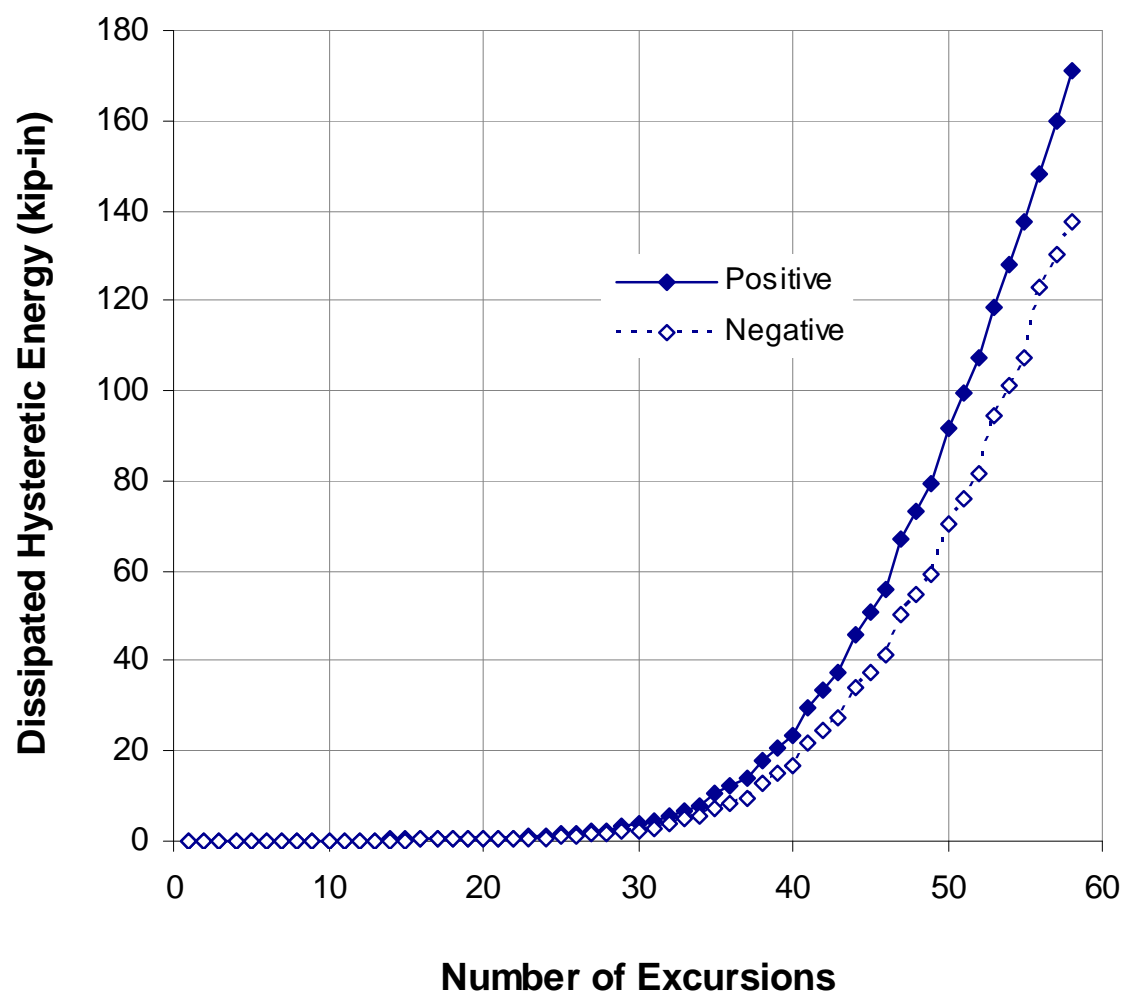

Figure 5-29: Dissipated Hysteretic Energy for Wall 1 


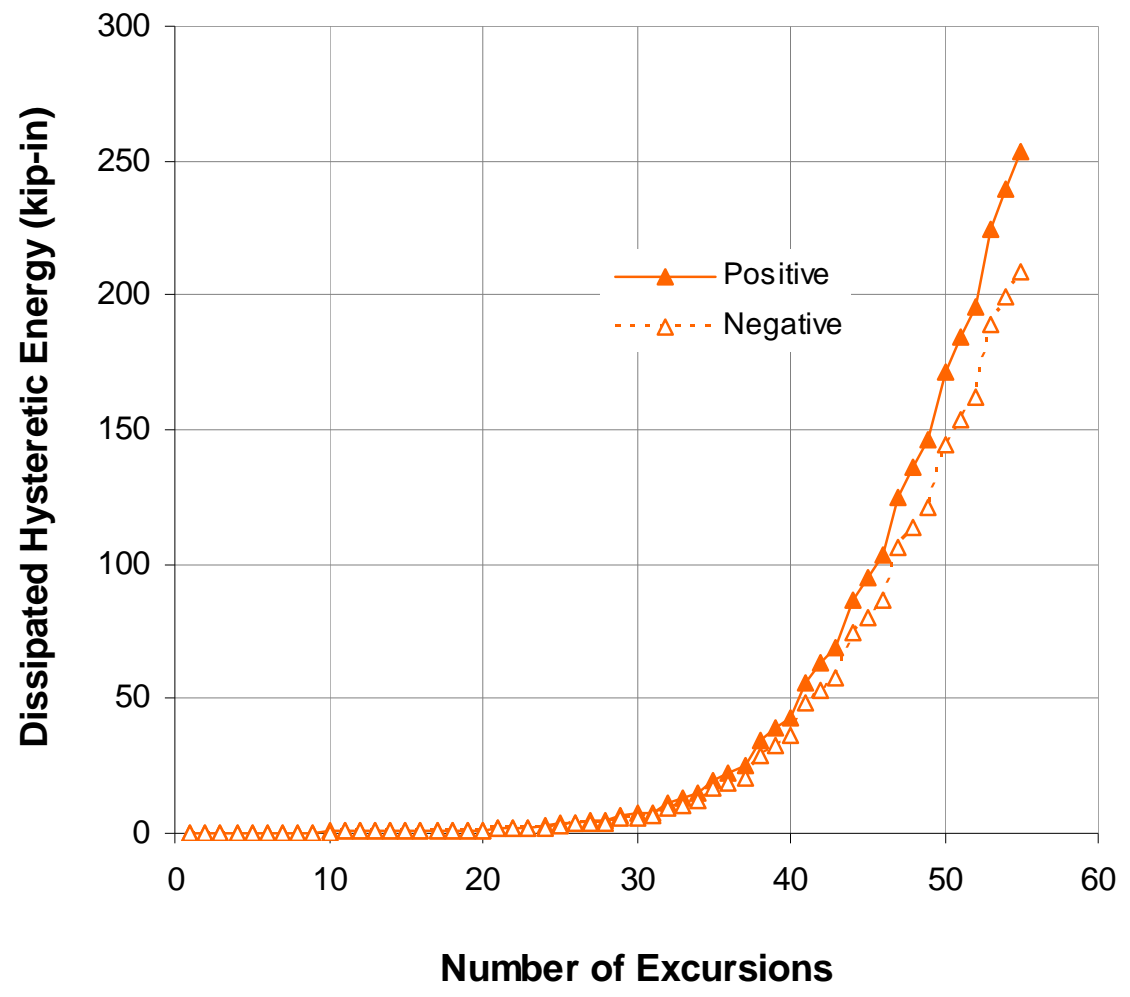

Figure 5-30: Dissipated Hysteretic Energy for Wall 2

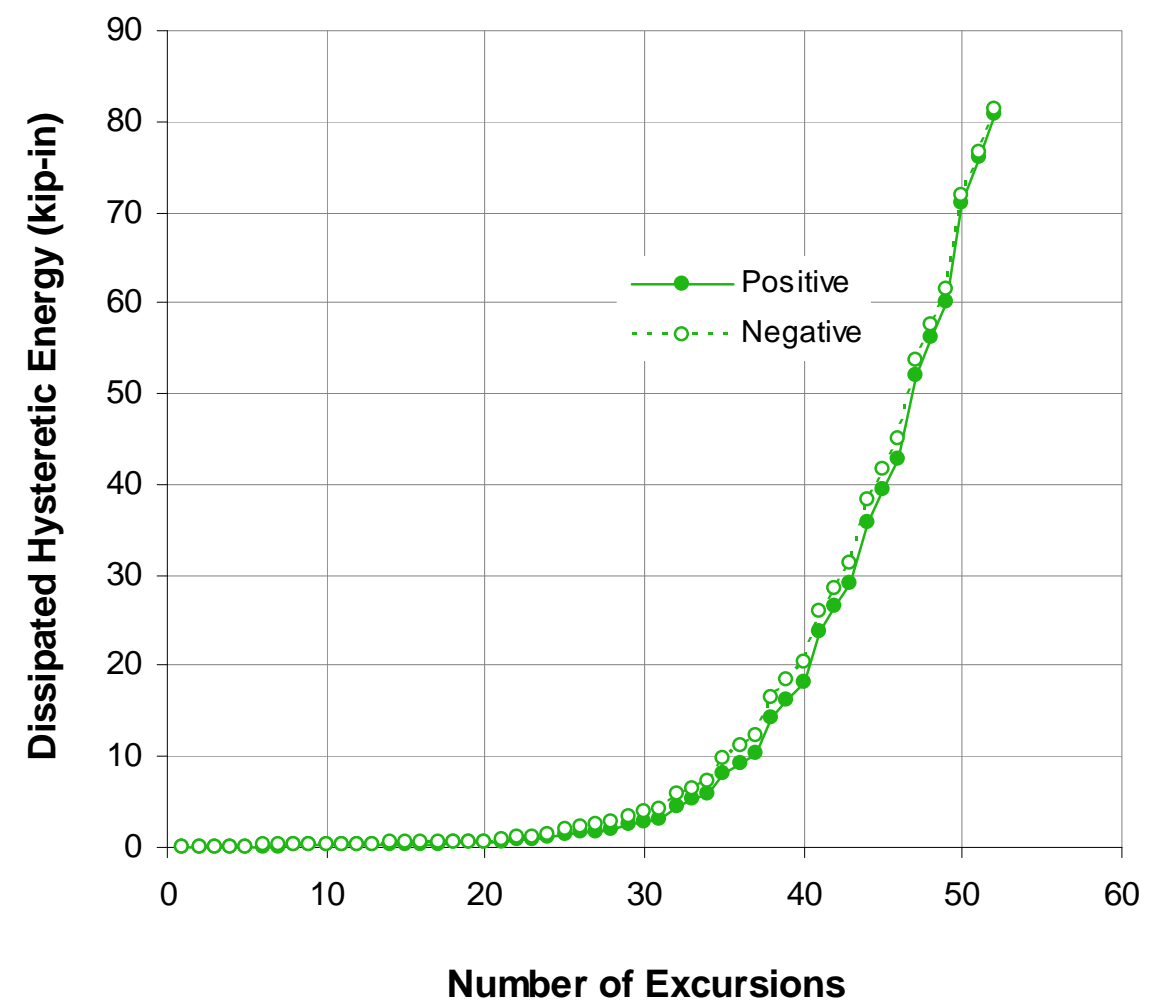

Figure 5-31: Dissipated Hysteretic Energy for Wall 3 


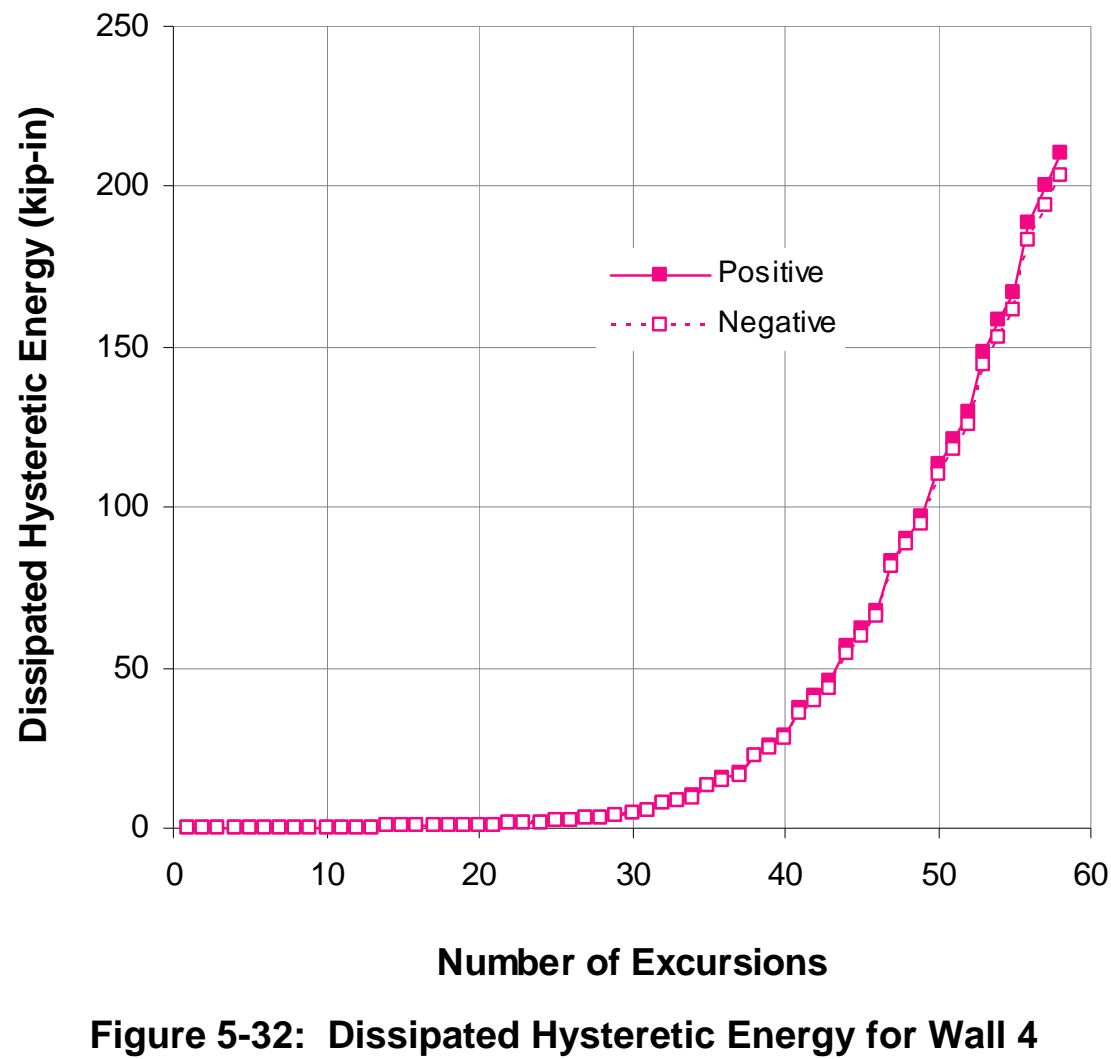

Ideally, the same amount of energy would be dissipated in the positive cycles as the negative. This would mean equal load application in each direction as well as symmetric cyclic degradation. Walls 1 and 2 exhibited more energy being absorbed on the positive section while Walls 3 and 4 showed nearly equal energy dissipation. This is likely due to the construction differences with the bare Wall 1 experiencing damage to the straw and having the negative cycles experiencing a weaker wall; while Wall 2 was so heavily detailed and rigid that damage may have occurred to the panels on the positive cycles and had therefore led to a weaker wall for the negative cycles. For comparison purposes, Figure 5-33 plots all four walls on the same set of axes. 


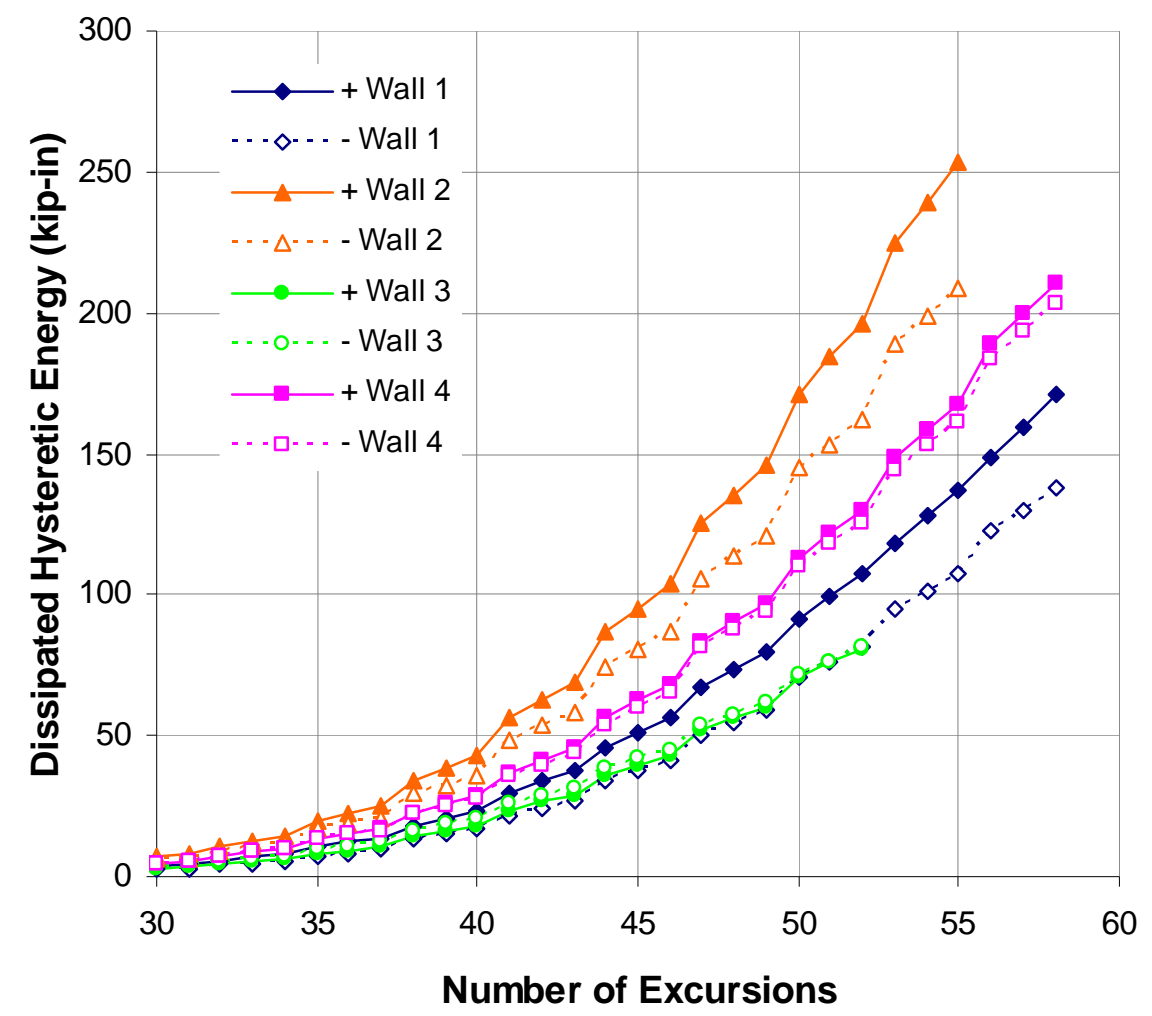

Figure 5-33: Dissipated Hysteretic Energy for All Wall Specimens

It is clearer in the figure above that Wall 2 (heavy detailing) absorbed the most energy with Wall 4 (sheathing on one side) coming in second. What is very interesting is that Wall 3 (wire rope with sheathing on one side) absorbed only as much energy as the negative cycles of Wall 1 (bare straw block). This means that in a seismic event, even though Wall 3 was sheathed with OSB, it would have dissipated as much energy as a damaged bare straw block wall. Of all of the points illustrated about the wire rope not performing as well as thread bar, this is the most significant from a shear wall design standpoint. The ability of this particular structural system to absorb and dissipate energy without incurring substantial damage at drift levels well past a yield point is a very important and compelling characteristic. In order to perform comparison between the 
Stak Block walls and other structural wall systems, the Normalized Hysteretic Energy per Ibarra et al. (2005) is shown in Figure 5-34.

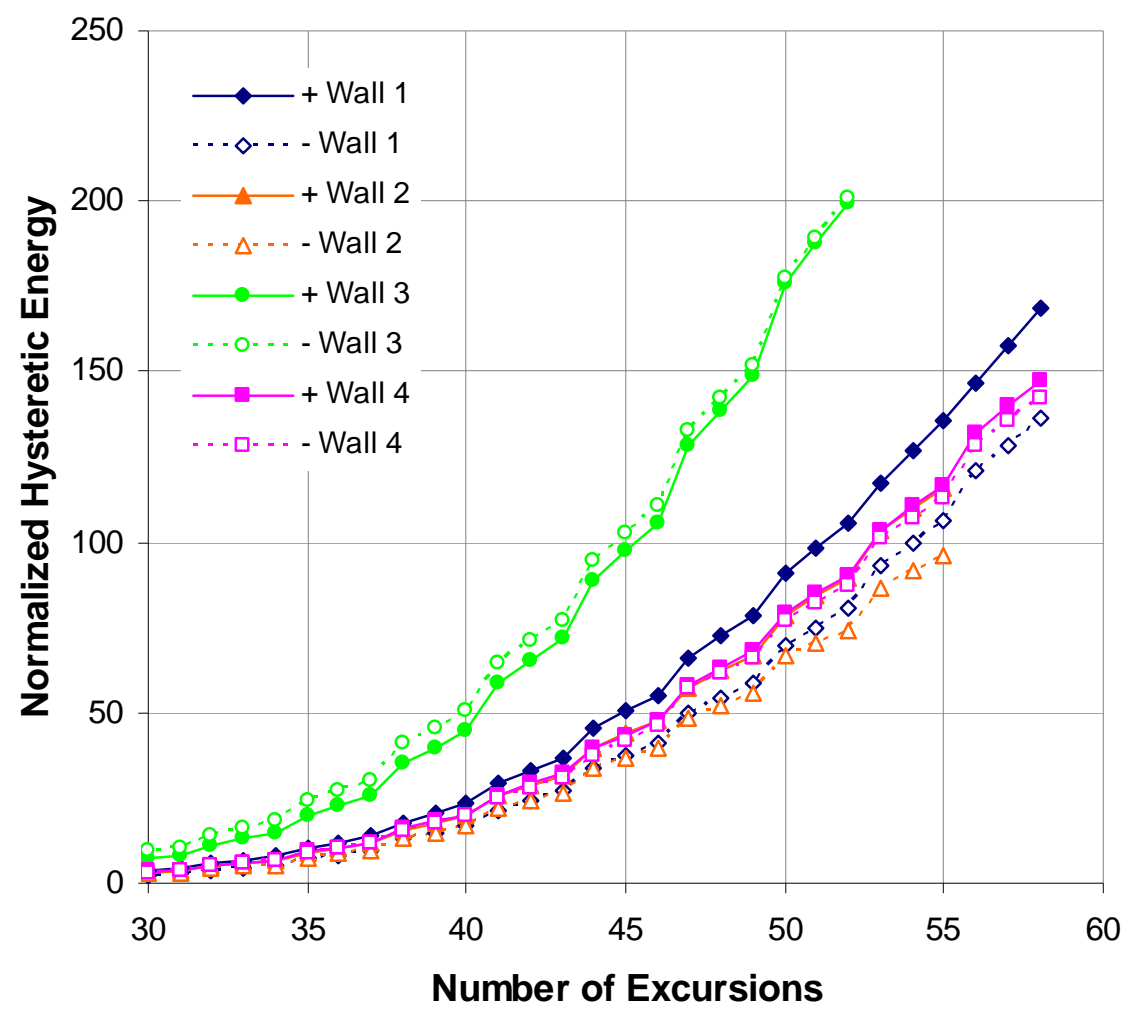

Figure 5-34: Normalized Hysteretic Energy for All Wall Specimens

It is of importance to note that Wall 3 had drastically higher NHE values (peaking near 200 versus 140). The use of wire rope versus thread bar is evident in the plot above with the three walls $(1,2$ and 4$)$ that used the thread bar being grouped closely together. While higher NHE values are a positive trait, it should be noted that Wall 3's high values are caused solely by that specimen's relatively low yield point. Since the NHE is calculated by dividing the yield force and displacement, a high NHE simply means the wall continued to dissipate energy without considerable damage past its yield at an early drift cycle. Dodge (2008) and Heredia (2010) performed an investigation into the hysteretic energy dissipated by their similarly detailed wood framed shear wall described 
in Section 5.8.3. Ibarra et al. (2005) tested a stronger and stiffer plywood sheathed wood framed shear wall and calculated the NHE to calibrate hysteretic models (in this case using the same CUREE-Caltech loading protocol). The reported results of their NHE analysis are shown below in Figure 5-35 through 5-37.

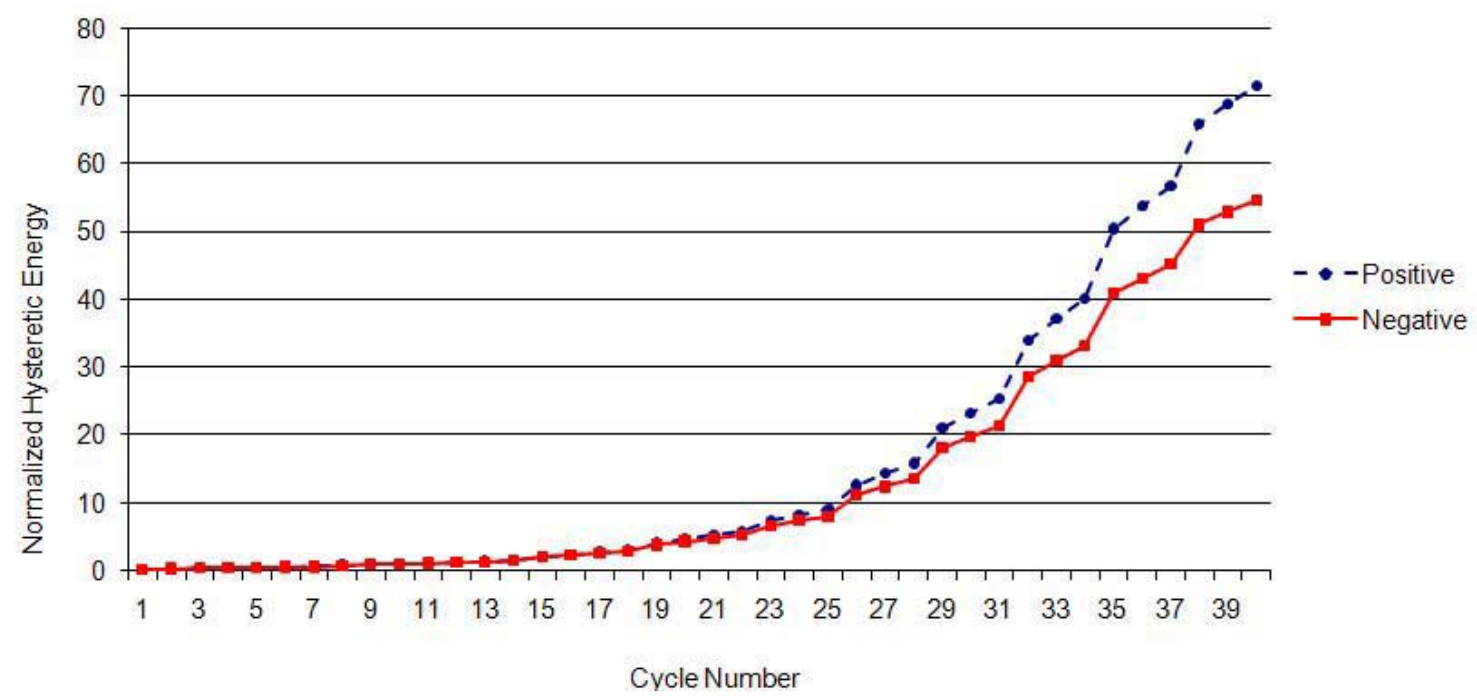

Figure 5-35: NHE for Wood Shear Walls from Dodge (2008)

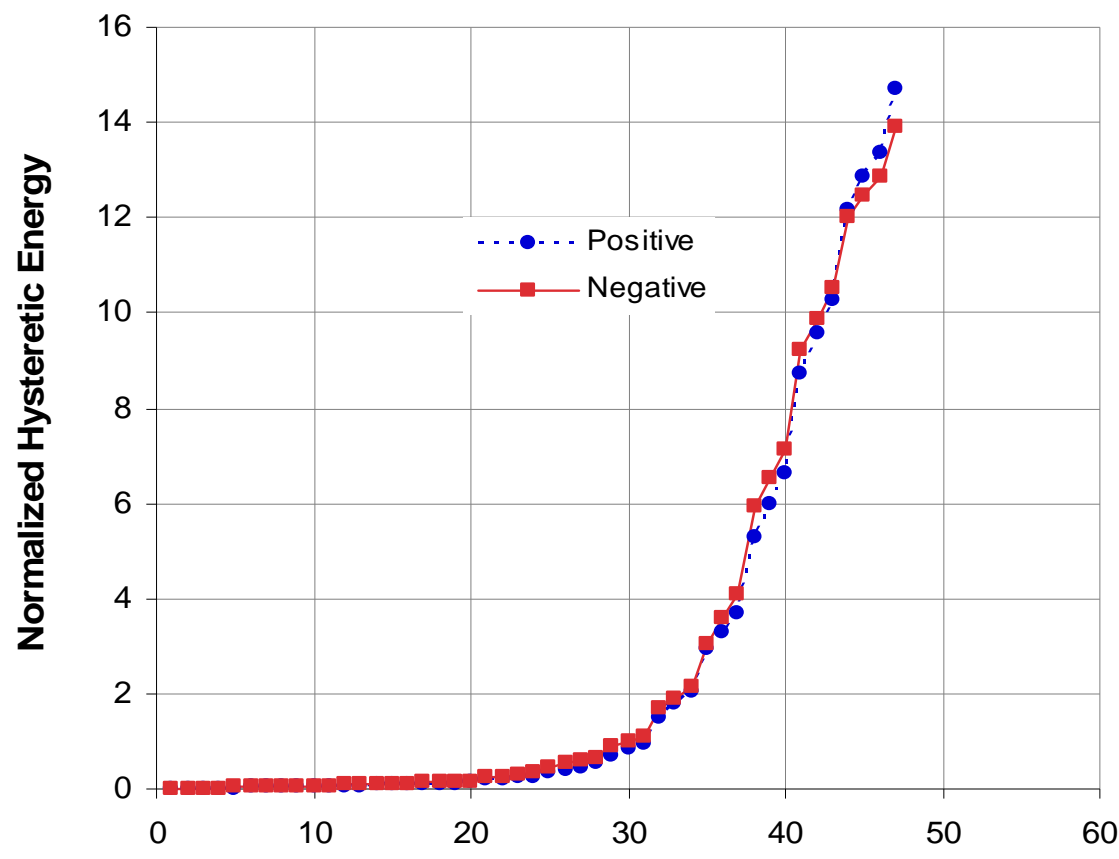

Number of Excursions

Figure 5-36: NHE for Wood Shear Walls from Heredia (2010) 


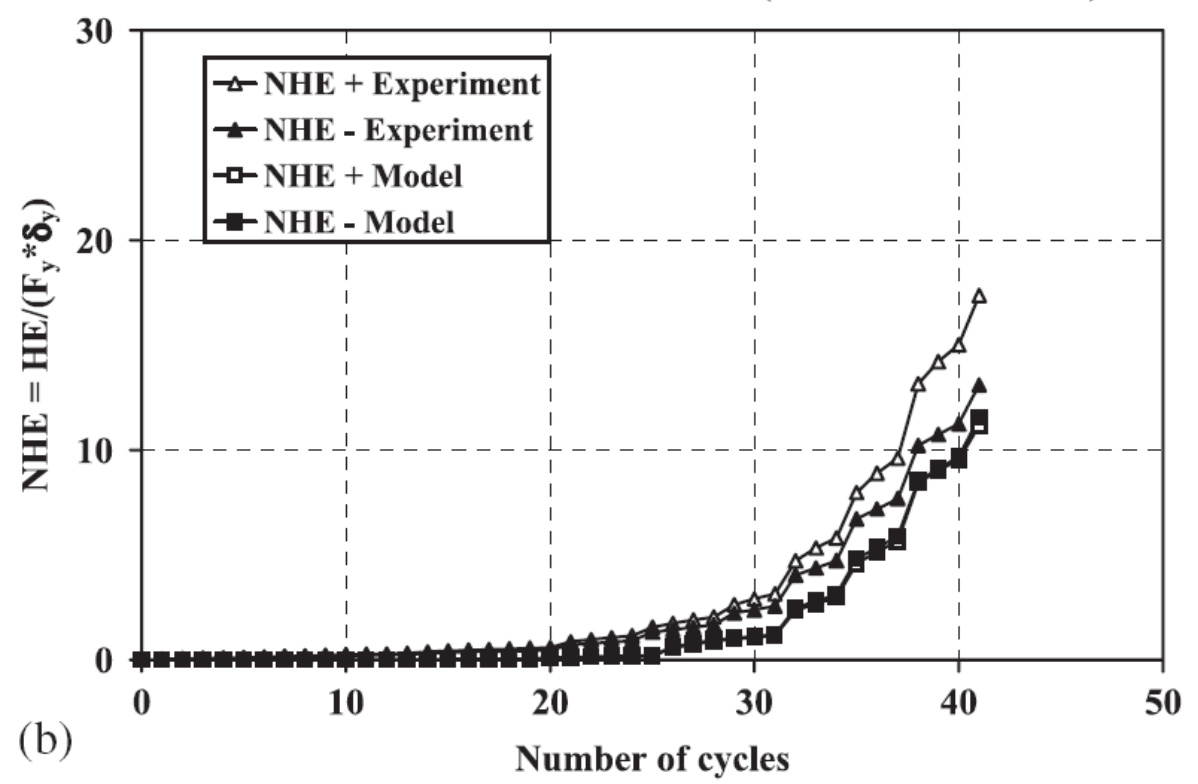

Figure 5-37: NHE for Wood Shear Walls from Ibarra et al. (2005)

It is interesting to note that the Normalized Hysteretic Energy values from

Dodge's study vary significantly from Heredia and Ibarra et al. Heredia and Ibarra et al. show similar values with Ibarra et al.'s being slightly higher due to the stiffer and stronger plywood used to sheath the wall specimen in lieu of Oriented Strand Board. Additionally, Gatto and Uang (2003) reported a "total absorbed energy" value of 16.2 $\mathrm{kNm}$ for their similarly detailed wall. This equates to $143 \mathrm{k}$-in of total dissipated energy for the positive and negative directions, and therefore approximately $72 \mathrm{k}$-in for each direction. This value is relatively close to those calculated by Heredia and Ibarra et al. for dissipated hysteretic energy.

The dissimilarity between Dodge's study and other stud framed walls could be a matter of a much lower yield force and displacement used to normalize the energy calculations. Dodge's yield force/displacement product $F_{y} * \delta_{y}$ was approximately 1.31 which is practically the same as for Wall 4, 1.36. Heredia calculated a much higher 
product of 4.83 as well as Ibarra with 7.0, which shows that where the yield point is interpreted in the data can greatly effect the NHE calculation. Additionally, the Normalized Hysteretic Energy values reported by Dodge are actually very close to the dissipated hysteretic energy values (i.e. not normalized) seen by Heredia as well as Gatto and Uang. This could be caused by the fact that Dodge's wall saw higher initial stiffness or the yield point could have been interpreted differently.

Despite these discrepancies it is important to note that at shear wall failure; Heredia obtained values of about 15 and 14, for positive and negative cycles, while Ibarra et al. reached 17 and 14, respectively. These values can be compared to the similarly detailed Wall 4 which reached 20 near the $40^{\text {th }}$ cycle (Figure 5-34). The Normalized Hysteretic Energy at failure from Dodge's report reaches values of approximately 72 for the positive cycles and 55 for the negative. Even with this dissimilarity with other woodframed wall studies, when comparing to Stak Block Wall 4, which went on to reach NHE values between 140 and 150 at the conclusion of testing, the superior ability to dissipate energy is evident. This NHE discrepancy of a factor of two or three as well as the similar positive and negative values for the Stak Block walls again underlines incredible ductility capacity of the straw walls when compared to timber stud framed walls. 


\section{Chapter 6 Development of Constitutive}

\section{Modeling}

For comparison purposes to similarly constructed timber framed shear walls, the parameters from a hysteretic model were determined from observed behavior of the fullscale shear wall tests. The variable of primary interest to compare straw block walls with stud framed walls was ductility. Ductility is the ability of a structure to deform into its inelastic range without a loss of lateral resisting force.

By performing a linear approximation of the backbone data and quantifying specific behavioral regions and critical points, a comparison to prior wood shear wall

testing could be performed. Additionally, code-prescribed ductility demand limit states could be calculated and used to quantify the ability of the Stak Block walls to deform well past their yield point. Finally, by comparing the results from the shear wall testing in this report to other shear wall investigations allowed for potential design strengths using various methodologies to be investigated. 


\subsection{Hysteretic Backbone Analysis}

\subsubsection{Linear Approximation of Backbone}

In order to simplify the data, the phenomenological backbones and forcedisplacement hysteresis curves were linearly approximated into segments representing elastic, yield, and post-yield branches. An example of this approximation and these branches is shown in Figure 6-1.

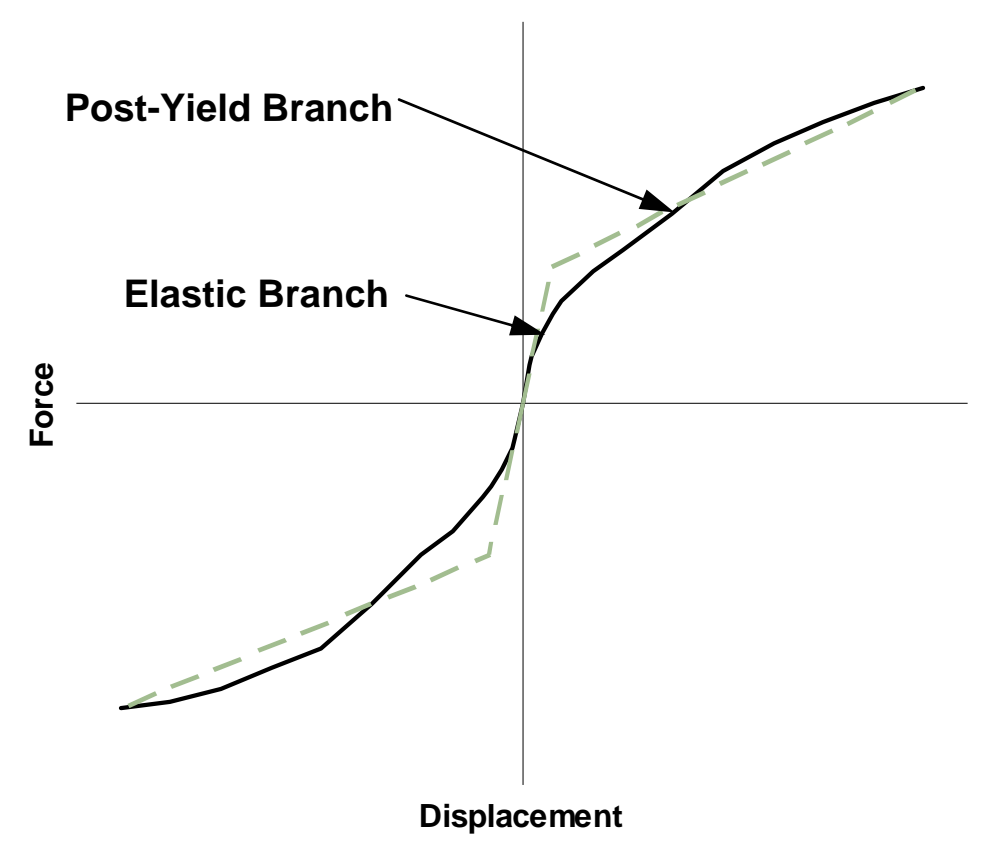

Figure 6-1: Linear Representation of Backbone Curve

Linearizing the backbone curves was accomplished by first visually matching the two slopes of the different branches and then fine-tuning the values to equate the areas under both curves. This process allowed for regains and critical points to be quantified and compared to other wall systems.

It is very important to note that none of the walls that were tested ever reached a true maximum, or peak, force-displacement value. As discussed earlier, although some damage to the corners of the shear panels was evident at the higher drift levels, the blocks 
themselves and the prestressing materials were still completely intact. Despite this, because of the lack of data for drift levels above $6.75 \%$, the last primary cycle is assumed to be the peak load and displacement. Some softening towards the end of testing was evident in the backbone curves, and this could have meant collapse or failure was imminent. Ibarra et al. (2005) show an idealized backbone curve that would apply to more brittle materials than the straw block walls. Figure 6-2 shows Ibarra et al.'s figure and the associated regions and critical points to be determined from testing.

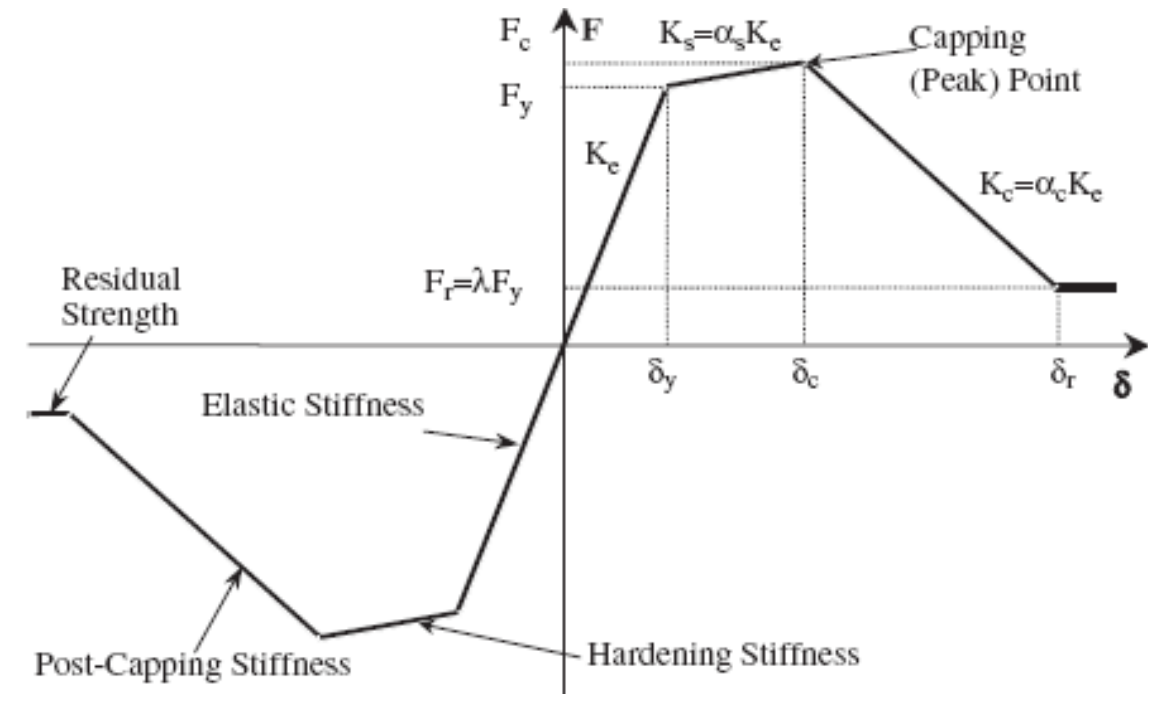

Figure 6-2: Example Backbone Curve for Typical Hysteretic Models

One major difference between Figure 6-2 and the backbones generated for the straw block walls is the length of the "hardening stiffness", or post-yield, region. Also, no post-capping stiffness or residual strength could be determined for the straw block walls. The behavior of the wall up to a drift level of $6.75 \%$ indicates that the force and displacement would increase indefinitely. A possible failure mechanism could be the fracture of the thread bars applying prestressing forces and wall stability. Despite this, drift levels this high for a structural system will likely never be experienced in a seismic event. 


\subsubsection{Backbone Characteristics}

The elastic and post-yield regions that were captured during testing can be compared to conventional shear wall systems. Below in Tables 6-1 and 6-2, the yield points, initial and hardening stiffnesses are shown for the positive excursions only; the negative values were very close and exhibited the same behavior as the opposite direction.

Table 6-1: Positive Backbone Characteristics

\begin{tabular}{ccccc} 
Specimen & $\begin{array}{c}\text { Yield } \\
\text { Strength } \\
\text { (kips) }\end{array}$ & $\begin{array}{c}\text { Peak } \\
\text { Strength } \\
\text { (kips) }\end{array}$ & $\begin{array}{c}\text { Elastic } \\
\text { Stiffness } \\
(\mathrm{k} / \mathrm{in})\end{array}$ & $\begin{array}{c}\text { Post-Yield } \\
\text { Stiffness } \\
(\mathrm{k} / \mathrm{in})\end{array}$ \\
\hline \hline Wall 1 & 1.81 & 4.63 & 4.90 & 0.70 \\
Wall 2 & 5.36 & 12.36 & 14.10 & 2.05 \\
Wall 3 & 2.00 & 5.38 & 5.00 & 1.00 \\
Wall 4 & 3.40 & 7.66 & 8.50 & 1.20
\end{tabular}

Table 6-2: Percentage Difference in Backbone Characteristics

\begin{tabular}{ccccc} 
Specimen & $\begin{array}{c}\text { \% Increase in } \\
\text { Yild Strength }\end{array}$ & $\begin{array}{c}\text { \% Increase in } \\
\text { Peak Strength } \\
\text { (kips) }\end{array}$ & $\begin{array}{c}\text { \% Increase in } \\
\text { Elastic Stiffness } \\
\text { (k/in) }\end{array}$ & $\begin{array}{c}\text { \% Increase in Post- } \\
\text { Yield Stiffness } \\
\text { (k/in) }\end{array}$ \\
\hline \hline Wall 1 & -- & -- & -- & -- \\
Wall 2 & $195.5 \%$ & $167.2 \%$ & $187.8 \%$ & $192.9 \%$ \\
Wall 3 & $10.3 \%$ & $16.3 \%$ & $2.0 \%$ & $42.9 \%$ \\
Wall 4 & $87.5 \%$ & $65.5 \%$ & $73.5 \%$ & $71.4 \%$
\end{tabular}

Table 6-2 uses the bare, unsheathed Wall 1 as a baseline for comparison purposes. On every level, from strength to stiffness, Wall 2, with sheathing on both sides, performed nearly three times as well as Wall 1. Wall 3 performed only slightly better than Wall 1 and had a nearly identical elastic stiffness, which is a very important value that relates to lower drifts which are likely only to be experienced during the lifetime of the structure. As expected, Wall 4 fell nearly halfway between Wall 1 and Wall 2 in 
terms of strength and stiffness. This all shows that the attachment of sheathing, level of detailing, and especially the type of prestressing used greatly effects the wall performance.

Ibarra et al. (2005) outlined parameters such as the strain-hardening ratio, $\alpha_{s}$, and the ductility capacity, $\delta_{c} / \delta_{y}$, which could be used to characterize a backbone curve for a given hysteretic model. These parameters obtained from the linearization of the backbone curves are found in Table 6-3.

Table 6-3: Positive Backbone Ductility Parameters

\begin{tabular}{ccccc} 
Specimen & $\begin{array}{c}\delta_{\mathrm{y}} \\
\text { (in) }\end{array}$ & $\begin{array}{c}\delta_{\mathrm{c}} \\
(\mathrm{in})\end{array}$ & $\begin{array}{c}\alpha_{\mathrm{s}} \\
\left(\mathrm{K}_{\mathrm{s}} / \mathrm{K}_{\mathrm{e}}\right)\end{array}$ & $\delta_{\mathrm{c}} / \delta_{\mathrm{y}}$ \\
\hline \hline Wall 1 & 0.37 & 4.86 & 0.14 & 13.14 \\
Wall 2 & 0.38 & 5.40 & 0.15 & 14.22 \\
Wall 3 & 0.40 & 4.80 & 0.20 & 12.01 \\
Wall 4 & 0.40 & 6.16 & 0.14 & 15.41
\end{tabular}

It is of import to note that all wall specimens yielded near the same displacement. Although the forces were drastically different, the same drift at which yield occurred could mean that it was a function of something physical in the blocks' geometry that initiated yielding. The sliding or slipping of the blocks typically occurred near the drift levels where the end of the elastic region was seen in data reduction. Another measure of the notable ductility of the prestressed wall system is the high ductility capacities, ranging from 12.0 to 15.4 .

\subsubsection{Comparison with Stud Wall Backbone Properties}

A similar study and hysteretic model was developed by Dodge (2008). Section 5.8.3 outlined the results of this report as well as the similar construction detailing (nail 
spacing and panel thickness) used for the shear wall specimens. Using the same CUREECaltech loading protocol on the same size wall in the exact same laboratory allow for a reasonable comparison between this report's testing results and those outlined in Dodge's thesis.

First, Dodge recorded a yield displacement and force of 0.26 inches and 5.13 kips, respectively. This compares to 0.38 inches and 5.36 kips for the heavily detailed Wall 2 and 0.40 inches and 3.40 kips for the single sided sheathing Wall 4 (the comparative results for Walls 2 and 4 will be reported in this order from herein). While the heavily detailed wall did perform better, this is to be expected as there were two sides of OSB panels resisting lateral force versus one. Also, the displacements at yield were one and a half times that seen in the stud framed walls. This translates directly to an increased elastic stiffness of the stud walls (20.0 kips/inch versus 14.4 kips/inch for the heavily detailed Wall 2). The initial stiffness discrepancy is even more evident in Wall 4 (8.5 kips/inch, or less than half).

Where the straw block walls performed considerably better than the timber shear walls was in the post-yield region. Dodge measured a stiffness of 0.92 kips/inch, while Walls 2 and 4 exhibited a much higher value of 2.05 and 1.20 kips/inch respectively. This much stiffer behavior beyond the elastic zone is also evident in the strain-hardening ratios ( 0.046 for stud framed walls versus 0.15 and 0.14 for the straw block walls). Similarly large ductility capacities were seen in the straw block walls versus stud framed specimens (14.22 and 15.41 versus 8.70). These values of $\delta_{c} / \delta_{y}$ show once again the superior ductility ability and supply in the prestressed straw block walls. 


\subsubsection{Peak-Oriented Hysteretic Model}

In developing a peak-oriented hysteretic model per Ibarra et al. (2005) for potential future dynamic modeling, other parameters were calculated to describe the behavior of the specimen beyond the backbone, or pushover, curve. Figure 6-3 shows the basic rules outlined by Ibarra for typical peak-oriented behavior.

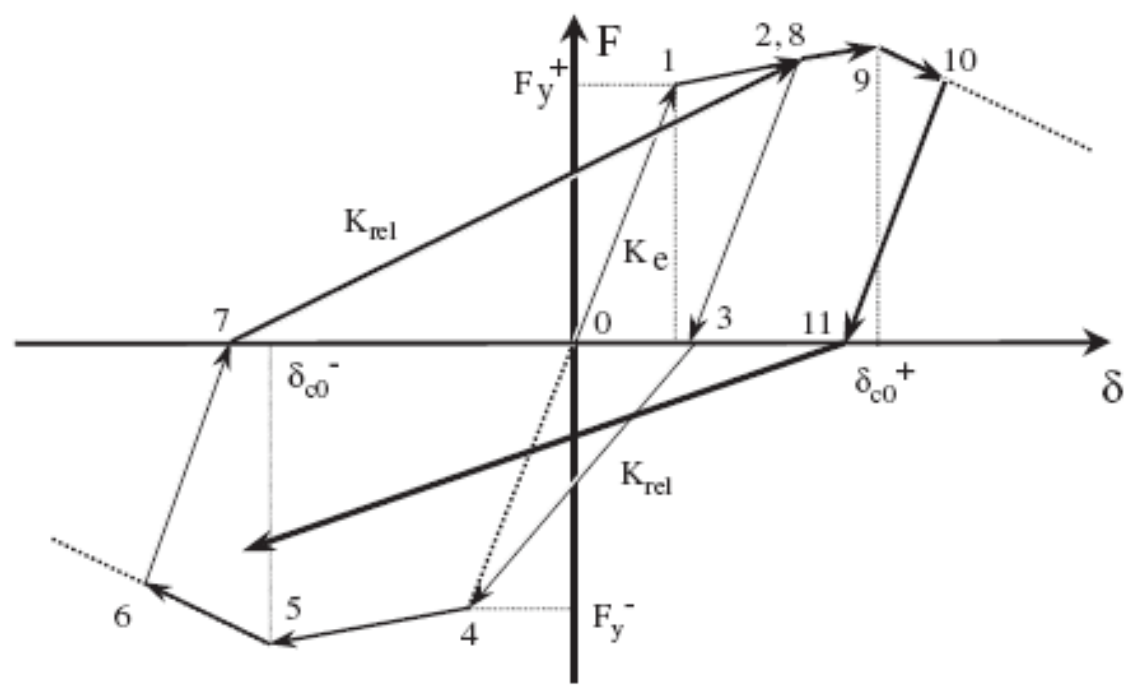

Figure 6-3: Basic Rules for Peak-Oriented Hysteretic Models

It can be seen that the unloading portion of this particular type of model is parallel to the initial elastic stiffness. It was observed in the hysteretic data that the unloading slope, $\mathrm{K}_{\mathrm{unl}}$, was inverse the elastic stiffness. Also, a parameter $\mathrm{K}_{\mathrm{rel}}$ was determined and approximated in a linear manner similar to the backbone curve. This reloading branch crossed the loading vertical axis at a point that will be termed $\mathrm{P}_{\mathrm{o}}$. These parameters are found below in Table 6-4. 
Table 6-4: Peak-Oriented Model Parameters

\begin{tabular}{cccc} 
Specimen & $\begin{array}{c}\mathrm{K}_{\text {rel }} \\
(\mathrm{k} / \mathrm{in})\end{array}$ & $\begin{array}{c}\mathrm{P}_{\mathrm{o}} \\
(\mathrm{kips})\end{array}$ & $\begin{array}{c}\mathrm{K}_{\mathrm{unl}} \\
(\mathrm{k} / \mathrm{in})\end{array}$ \\
\hline \hline Wall 1 & 0.31 & 1.27 & -4.90 \\
Wall 2 & 0.71 & 1.82 & -14.10 \\
Wall 3 & 0.39 & 0.55 & -5.00 \\
Wall 4 & 0.46 & 1.16 & -8.50
\end{tabular}

The values for the reloading stiffness range from 35 to $44 \%$ of the post-yield stiffnesses from before. This is slightly different than Ibarra's hypothetical model where the reloading stiffness appears to be higher than the post-peak. Strength degradation accounts for this loss by adjusting the model to reload to a force that was not quite as high as the previous excursion along the backbone. The fact that the wall specimens consistently had residual force (manifesting itself as $\mathrm{P}_{\mathrm{o}}$ ) at zero displacement is likely due to the presence of resistant prestressing forces.

\subsection{Description of Hysteretic Model}

Although a full dynamic modeling computer analysis was outside of the scope of this study, a basic investigation into the hysteretic behavior of Stak Blocks was performed using already developed models developed for wood-framed walls. A peakoriented hysteretic model with strength degradation would likely be used in modeling the dynamic behavior of the straw block walls. The peak-oriented model, as described by Ibarra et al. (2005), appears to be the most appropriate because the walls did not approach a "break point” as typically seen in a pinched hysteretic model; instead the walls' reloading path always targeted the previous maximum displacement. While the walls did 
not exhibit cyclic stiffness degradation, some strength degradation was evident in the first secondary drift cycle following each primary cycle.

The deterioration of the shear walls' properties was caused by the dissipation of system hysteretic energy as discussed above in Chapter 5. This deterioration is typically quantified by a single factor, $\beta$, calculated as shown in Equation 6-1.

\section{Equation 6-1: Cyclic Deterioration Parameter, $\beta$}

$$
\beta_{i}=\left(\frac{E_{i}}{E_{t}-\sum_{j=1}^{i} E_{j}}\right)^{c}
$$

Where: $E_{i}=$ Hysteretic Energy per Excursion, $i$

$$
\begin{aligned}
& E_{t}=\gamma F_{y} \delta_{y}=\text { System Hysteretic Energy Capacity } \\
& \Sigma E_{j}=\text { Sum of Hysteretic Energy in All Previous Excursions } \\
& c=\text { Factor for Rate of Deterioration } \\
& F_{y}=\text { System Yield Strength } \\
& \delta_{y}=\text { Displacement at Yield }
\end{aligned}
$$

Although Ibarra et al. define deterioration parameters for yield strength, post-yield stiffness, post-peak stiffness, and post-peak reference strength, only the deterioration in yield strength was seen in this study. Equation 6-2 shows the relationship between $\beta_{s}$ (the subscript "s" for strength) and the measured forces.

Equation 6-2: Positive and Negative Excursion Yield Force Deterioration

$$
\begin{aligned}
& F_{i}^{+}=\left(1-\beta_{s, i}\right) F_{i-1}^{+} \\
& F_{i}^{-}=\left(1-\beta_{s, i}\right) F_{i-1}^{-}
\end{aligned}
$$

Where: ${F_{i}}^{+/-}=$Positive or Negative Yield Force of Current Cycle

$$
\mathrm{F}_{\mathrm{i}-1}^{+/-}=\text {Positive or Negative Yield Force of Previous Cycle }
$$


In materials such as wood studs, the hysteretic energy is dissipated by damage in the form of splitting of wood grains and slipping of nails. In the case of these straw blocks, although damage occurred to the OSB panels, sliding of nails and screws, the sliding of the blocks also absorbed a considerable amount of system energy. In order to have the deterioration model fit reasonably close the Rate of Deterioration Factor, c, in Equation 6-1 must be small. Rahnama and Krawinkler (1993) suggested an appropriate range of 1.0 to 2.0. This range holds true for traditional materials such as wood and concrete where once damage occurs, it is irreversible.

In the case of the straw block walls, practically zero force deterioration was seen between subsequent secondary cycles. This is an important property of the straw block walls' behavior: the ability to deform well past a yield point and have little to no strength degradation and no loss in stiffness would be vital in terms of seismic ductility.

\subsection{Discussion of Ductility Demand Limit States}

Unlike other investigations into wood shear walls where failure and collapse occurred in testing, assumptions must be made in developing a hysteretic model. Drift levels beyond those prescribed by the CUREE-Caltech loading protocol were not explored during testing because they were deemed structurally unrealistic. A drift of $8 \%$, which still may not reach the peak of the wall due to the nature of the prestressing interacting with the soft straw blocks, would translate to a lateral displacement of 10 inches for a 10 foot tall structure. A drift this high would likely mean the complete loss of any attached structural or architectural systems. For instance, non-structural materials such as windows and interior finishes would be completely destroyed by a drift ratio this high. What would be more of a drastic failure is the attached roof framing and any 
adjacent floors. As a whole, buildings simply cannot be designed to resist differential lateral displacements of this magnitude.

\subsubsection{ASCE/SEI 41 Limit States}

A performance-based evaluation of the effectiveness of straw block walls was accomplished using the ASCE/SEI 41 (2007) limit states. Immediate Occupancy (IO) of a building is defined in seismic rehabilitation as having no permanent drift, retaining its original strength and stiffness and exhibiting only minor cracking of nonstructural components. In testing, this point is where permanent, visible damage occurs in the experiments, but not greater than 0.67 times the Life Safety limit.

Life Safety (LS) is defined as having some residual strength left in all stories, with gravity-load-bearing elements retaining functionality, but no out-of-plane failure of walls. Some permanent drift may have occurred and many architectural, mechanical, and electrical systems damaged beyond economical repair. In experimental results, this point is defined as 0.75 times the peak deformation on the backbone curve.

The Collapse Prevention (CP) performance level is when little residual stiffness and strength is remaining, but load-bearing columns and walls still function. Large permanent drifts are seen with some exits blocked because of extensive damage and the building is basically near collapse. This point is defined as the peak deformation on the backbone curve, but not greater than 0.75 times the deformation immediately prior to collapse on the residual strength branch. Figure 6-4 shows various types of wall behavior put forth by ASCE/SEI 41. 


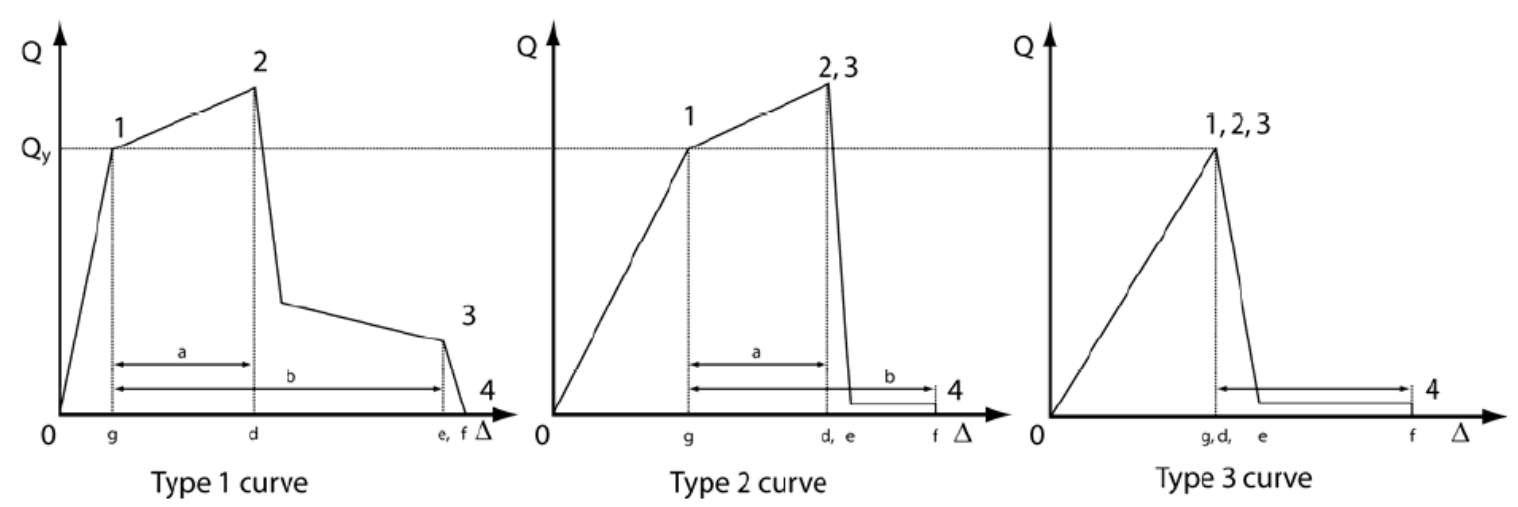

Figure 6-4: Types of Nonlinear Wall Responses

The Type 1 curve is characterized by ASCE/SEI 41 and FEMA 440 as Ductile or Deformation Controlled, Type 2 as Semi-ductile, and Type 3 as Brittle or Force Controlled. As iterated earlier in this report, no post-peak region was captured during testing, so the residual branch is unknown.

The linear representations of the backbones in this study do more closely match the relative slope differences between regions seen in Type 1, so the curves were classified as this. Also, it can be assumed that because of the prestressed wall, even after failure, the straw block walls should exhibit considerable residual force resistance.

Figure 6-5 presents the deformation acceptance criteria relative to one another; Table 6-5 presents the calculated limit states, normalized to the yield deformation. 


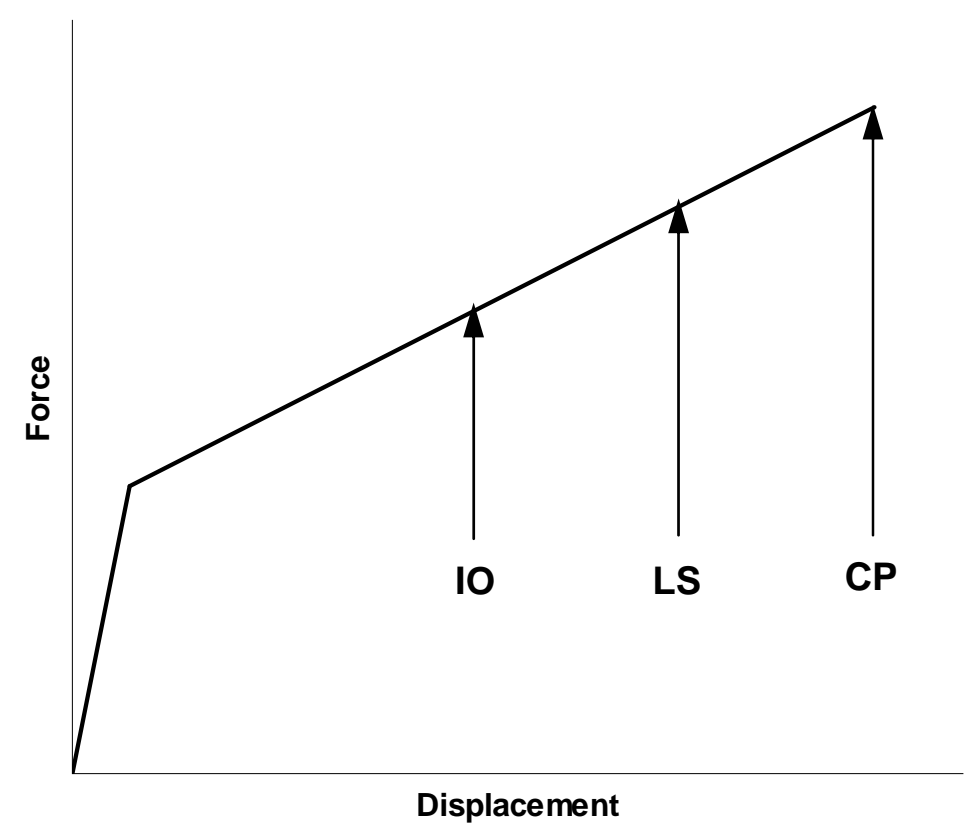

Figure 6-5: Capacity Curve with ASCE/SEI 41 Performance Limit States

Table 6-5: Calculated Ductility Demand Limit States per ASCE/SEI 41

\begin{tabular}{cccc} 
Specimen & $\begin{array}{c}\text { Immediate } \\
\text { Occupancy }\end{array}$ & $\begin{array}{c}\text { Life } \\
\text { Safety }\end{array}$ & $\begin{array}{c}\text { Collapse } \\
\text { Prevention }\end{array}$ \\
\hline \hline Wall 1 & 6.60 & 9.85 & 13.14 \\
Wall 2 & 7.14 & 10.66 & 14.22 \\
Wall 3 & 6.03 & 9.00 & 12.01 \\
Wall 4 & 7.74 & 11.56 & 15.41
\end{tabular}

In this case, the lack of post-peak information or data conservatively skews the limit states. If the residual branch had been captured, the Collapse Prevention deformation could be taken immediately prior to failure, as shown as point 3 in Figure 64. Instead, the peak-point was taken as the Collapse Prevention performance level and this in turn affected the other two levels which were calculated from this CP value. Despite this, calculating these limit states again shows the considerable ductility 
exhibited by this wall system, especially for Immediate Occupancy and Life Safety limits.

Dodge (2008) reported ductility demand limit states of 3.81, 5.69 and 12.09, respectively, for their similarly detailed stud framed wall. Although the Collapse limit is relatively close, the much higher (approximately 100 percent greater) calculated limit states for Immediate Occupancy and Life Safety show the increased ductility supply available in this system when compared with conventional construction materials and methods.

\subsection{Potential Shear Wall Design Strengths}

In developing a new building material such as Stak Block, a vital characteristic or finding would be the lateral design strength for engineers and architects in industry to use. While it would be ideal to supply this design value, the fact remains that only four full-scale shear wall tests have been performed and only one for each variable. Until further refinements of typical construction detailing and methods are developed and tested and more rigorous non-linear simulations are performed, the findings of this study will act to demonstrate the feasibility of using Stak Blocks as a load bearing and lateral force resisting system and to provide estimates of the lateral design strengths one would expect to achieve using Stak Blocks.

It is outside the scope of this investigation to perform the additional wall testing and detailed dynamic modeling needed to safely establish design shear values for the Stak Block walls. Despite this, the findings of this study will allow for some preliminary strength values to be determined by comparing to a code-accepted system such as stud framed shear walls. The following are three relatively simple ways the data can be 
evaluated to determine some approximate design values to be refined with further testing and analysis.

\subsubsection{Drift at Design Strength Approximation}

The first methodology used to investigate potential design shear strengths was to use the code-prescribed allowable shear strengths to estimate an acceptable drift level. This analysis assumes that the drift levels corresponding with the allowable shear forces would take into account damage to attached non-structural finishes and windows. The allowable shear strength from IBC 2006 Table 2306.4.1 (International Code Council, 2006), assuming $1 / 2$ inch OSB sheathing attached with $8 d$ nails spaced at 4 inches along the panel edges, was 430 plf. These are design details most similar to the walls constructed and tested by Dodge, Gatto and Uang, and Heredia. The design level shear force over an 8-foot length of wall would in turn be 3,440 lbs. With this design load and the backbone data, the drift level for each wall was determined as shown in Figure 6-6. Table 6-6 summarizes the drift levels for each wall corresponding to this design load. Using the mean drift of $0.45 \%$ determined from the data from Dodge, Gatto and Uang, and Heredia, the corresponding strengths for each of the Stak Block walls was then determined, also shown in Table 6-6.

It should be noted that Dodge's backbone reached the code prescribed shear at a much lower drift due to the higher initial stiffness seen in his wall testing. If his data were used alone, the result would be lower design strengths for the Stak Block walls. 


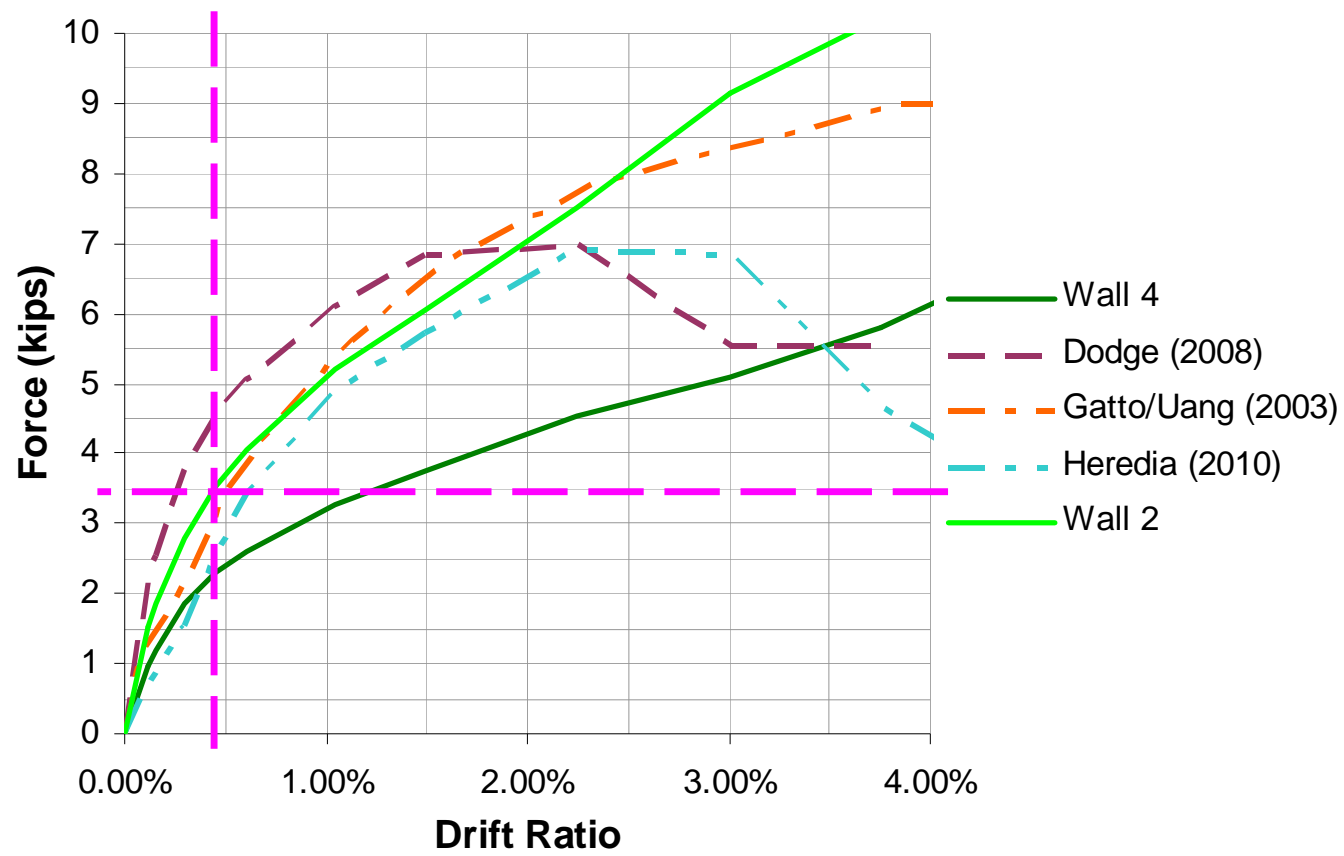

Figure 6-6: Backbone Comparison @ Code-Allowable Shear

Table 6-6: Design Shear based on Drift @ Code-Allowable Shear

\begin{tabular}{cccc} 
Specimen & $\begin{array}{c}\text { IBC Design Value } \\
\text { (plf) }\end{array}$ & $\begin{array}{c}\text { Drift @ Design } \\
(\%)\end{array}$ & $\begin{array}{c}\text { Factored Strength } \\
\text { (plf) }\end{array}$ \\
\hline \hline Wall1 & -- & -- & 189 \\
Wall2 & -- & -- & 442 \\
Wall3 & -- & -- & 175 \\
Wall4 & -- & -- & 287 \\
Dodge (2008) & 430 & 0.25 & -- \\
Heredia (2010) & 430 & 0.6 & -- \\
Gatto/Uang (2003) & 430 & 0.5 & --
\end{tabular}

\subsubsection{Force at 2.5\% Drift Approximation}

The IBC (International Code Council, 2006) and ASCE 7 (American Society of Civil Engineers, 2006) impose maximum allowed drift limits of 2.5\% on structures that are four stories or less in Occupancy Categories I and II. Stak Blocks are intended to be used on these smaller scale residential structures, so this drift limit is applicable. To 
apply the Ductility Demand limit states from Section 6.3, the 2.5\% drift limit is analogous to Life Safety, with Immediate Occupancy occurring near 1.5\% drift. Also, as the methodology employed in section 6.4 .1 was considering drift levels of only $0.45 \%$, it appears it may not be the most appropriate method of analysis looking at behavior well below design level drifts.

The following methodology used to approximate design shear strength based on 2.5\% drift levels and would be considered a Life Safety prescription. This calculation is done by obtaining the force per unit length from the backbone data corresponding to 2.5\% drift as shown in Figure 6-7.

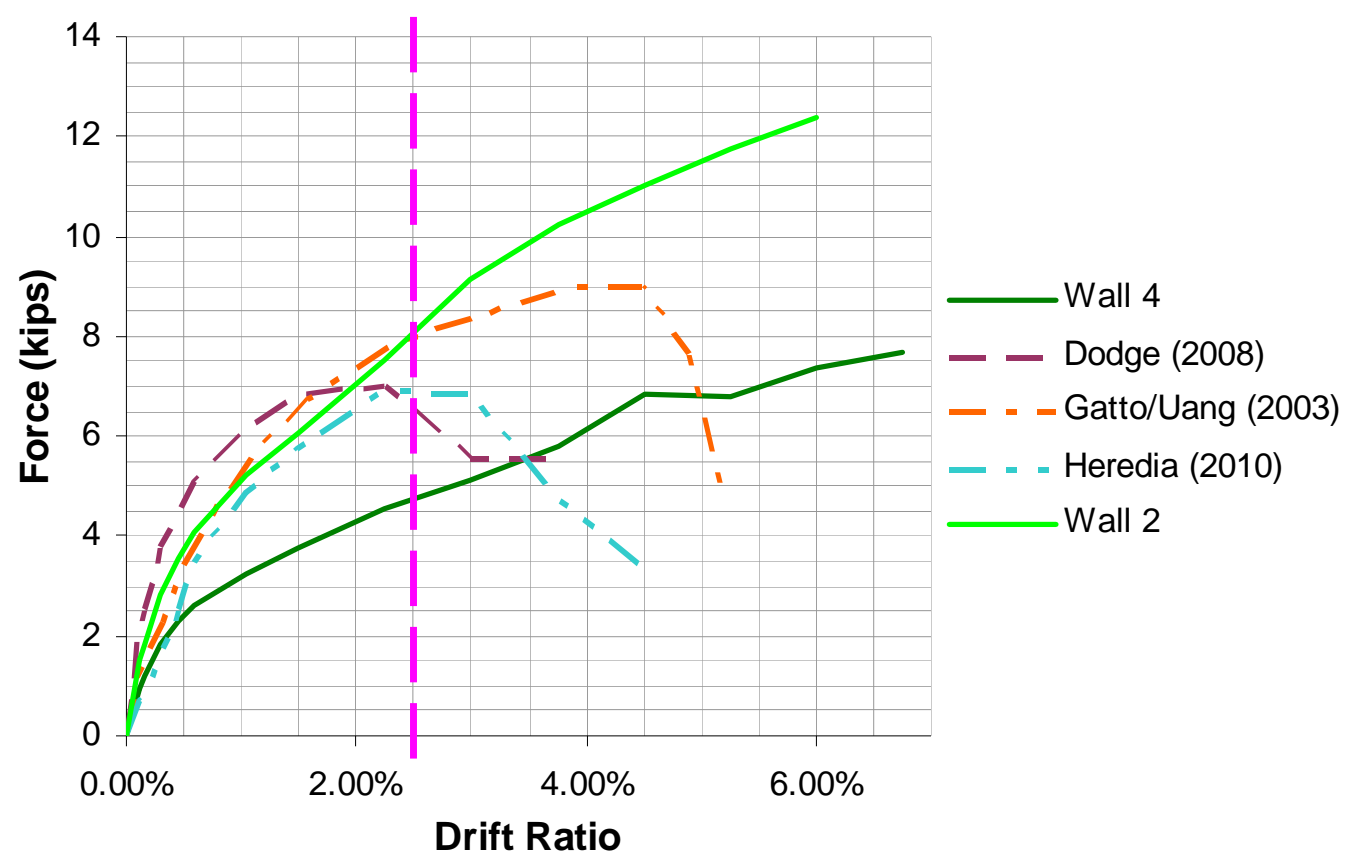

Figure 6-7: Backbone Comparison @ 2.5\% Drift

As discussed in section 6.4.1, the IBC allowable shear strength for the walls reported by Dodge, Gatto and Uang, and Heredia was 430 plf. Dividing the load/ft corresponding to $2.5 \%$ drift in Figure 6-7 by the IBC allowable shear strength provides 
the design factors of safety for Dodge (2008), Gatto and Uang (2003), and Heredia (2010). Table 6-7 below outlines the findings of this approximation.

Table 6-7: Design Shear based on Forces @ 2.5\% Drift

\begin{tabular}{|c|c|c|c|c|}
\hline Specimen & $\begin{array}{l}\text { Force per } \\
\text { Unit Length } \\
\text { (plf) }\end{array}$ & $\begin{array}{l}\text { IBC Design } \\
\text { Value } \\
\text { (plf) }\end{array}$ & $\begin{array}{c}\text { Factor of } \\
\text { Safety }\end{array}$ & $\begin{array}{l}\text { Factored } \\
\text { Strength } \\
\text { (plf) }\end{array}$ \\
\hline Wall1 & 388 & -- & -- & 187 \\
\hline Wall2 & 1000 & -- & -- & 482 \\
\hline Wall3 & 450 & -- & -- & 217 \\
\hline Wall4 & 594 & -- & -- & 286 \\
\hline Dodge (2008) & 813 & 430 & 1.89 & -- \\
\hline Heredia (2010) & 863 & 430 & 2.01 & -- \\
\hline Gatto/Uang (2003) & 1000 & 430 & 2.33 & -- \\
\hline
\end{tabular}

The mean factor of safety between the three wood shear wall investigations was 2.07. The factored strengths for Walls 1 through 4 were determined by dividing force/unit length corresponding to $2.5 \%$ drift by this mean factor of safety; the corresponding factored strengths for Stak Block Walls 1 through 4 are provided in Table 6-7. Comparing the results of the method of this section to those determined using the methodology in section 6.4.1, the results are very similar. The two methods give virtually identical results for factored strengths for Walls 1 and 4, while the factored strength for Wall 2 is 9\% larger using the method of this section as compared to the method of section 6.4.1. The largest discrepancy between the two methods is associated with Wall 3 with a net difference of $24 \%$.

\subsubsection{Hysteretic Energy Dissipated at 2.5\% Drift Approximation}

The third method used to approximate potential design shear strengths was to look at the dissipated hysteretic energy by the wall system up to the same $2.5 \%$ drift limit. Using the dissipated hysteretic energy plots from Section 5.9, the amount of energy 
dissipated in kip-inches could be determined at the $2.5 \%$ drift level. This value was then compared to the allowable shear strengths from IBC 2006 to obtain a ratio. In this case, Gatto and Uang did not supply detailed hysteretic energy plots and calculations, so data from Ibarra et al. (2005) was used for comparison purposes. Table 6-8 below outlines the findings of this approximation.

Table 6-8: Design Shear based on Dissipated Energy @ 2.5\% Drift

\begin{tabular}{cccccc} 
Specimen & $\begin{array}{c}\text { Hist Energy/ } \\
8 \text { feet } \\
\text { (kip-in/8 feet) }\end{array}$ & $\begin{array}{c}\text { Hist } \\
\text { Energy/foot } \\
(\mathrm{lb}-\mathrm{ft} / \mathrm{ft})\end{array}$ & $\begin{array}{c}\text { IBC Design } \\
\text { Value } \\
(\mathrm{lb} / \mathrm{ft})\end{array}$ & $\begin{array}{c}\text { Strength/Energy } \\
\left(\mathrm{ft}^{-1}\right)\end{array}$ & $\begin{array}{c}\text { Factored Strength } \\
(\mathrm{plf})\end{array}$ \\
\hline \hline Wall1 & 15.6 & 163 & -- & -- & 236 \\
Wall2 & 31.6 & 329 & -- & -- & 476 \\
Wall3 & 15.3 & 159 & -- & -- & 230 \\
Wall4 & 22.5 & 234 & -- & -- & 339 \\
Dodge (2008) & 31.0 & 323 & 430 & 1.33 & -- \\
Heredia (2010) & 27.1 & 282 & 430 & 1.52 & -- \\
Ibarra (2005)* & 29.8 & 310 & 460 & 1.48 & -
\end{tabular}

The first assumption made in this calculation was with the energy values from Dodge (2008). As discussed in Section 5.9, there appears to be a large discrepancy in the NHE values reported when compared to similar wood framed walls. Because of this, the Normalized Hysteretic Energy reported was assumed to be the dissipated hysteretic energy for the purposes of this calculation. The next assumption was with the construction detailing used by Ibarra et al. (2005); the report only indicated that plywood was used for the wood specimens, so it was assumed that the plywood was $1 / 2$ inch thick with $8 d$ nails spaced at 4 inches along the panel edges. The allowable strength per IBC 2006 Table 2306.4.1 for this assumption is 460 plf.

The average strength-to-energy-dissipated ratio per foot length of wall for the three wood studies was $1.45 \mathrm{ft}^{-1}$. All three values were quite close which indicates the assumption made about Dodge’s data may have been correct. Additionally, the design 
strength approximation values were reasonably close to those found above in Section

6.4.1 and 6.4.2; on average, they were $16 \%$ higher than the averages and ranged from $3 \%$ higher for Wall 2 to 26\% higher for Wall 1 as compared to the of the mean design strengths determined in sections 6.4.1 and 6.4.2 values.

\subsubsection{Design Strength Approximation Results}

As iterated before, without the necessary additional testing and modeling that are outside of the scope of this report, any values being reported should be taken as approximations. Ideally, future research will corroborate these values and design strengths for different wall configurations can be more confidently determined.

As discussed in section 6.4.3, using the dissipated hysteretic energy at 2.5\% drift yielded significantly higher values than the prior two methods. This is caused by the lower initial stiffnesses seen in Stak Block walls when compared to timber framed walls, which in turn led to lower strengths when back-calculated from force or displacement parameters.

Section 5.9 outlined the superior ability of the Stak Block walls to dissipate energy by means of block sliding, panel damage, and screw/nail slippage. All of these very ductile failure mechanisms allow for the wall to deflect well into its inelastic range without the loss of lateral resisting force. For example, Wall 1 saw the greatest increase in design strength in section 6.4.3, which is likely caused by the sliding of the blocks being the main source of energy dissipation due to the absence of sheathing. This ability to effectively dissipate energy at lower drift levels, even with a lower stiffness, is evidenced by the higher strength approximations seen with the final method. 
As shown in the backbone comparison from Section 5.8, Wall 2 performed as well, if not better than wood framed walls with sheathing on one side. This is shown again with the factored strength values of around 460 being slightly greater than those same wood framed walls. Also, the factored strengths for Wall 3 and Wall 4 are drastically different (around 200 plf versus 300 plf respectively) even though the walls were constructed exactly the same, with the only difference being the method of prestressing. Again this shows the performance issues associated with the switch to wire rope from thread bar. For comparison purposes, the IBC allowable shear strength of a 2x4 stud wall with $5 / 16$ inch thick OSB sheathing attached with 6 d nails spaced at 6 inches along the edges is 200 plf. Wall 4, with thread bar and sheathing on one side, has preliminary shear strength of nominally 300 plf. This compares to 280 plf for a wood framed wall sheathed on one side with $1 / 2$ inch OSB fastened with $8 d$ nails at 6 inches along the edges.

Further investigations including testing of different screw/nail spacing as well as various thicknesses and types of sheathing will provide more insight into possible design values for Stak Block wall systems. This report sought to develop construction detailing as well as basic lateral force resisting behavior and the shear strengths indicated in Tables 6-6 through 6-8 will hopefully be corroborated by future modeling and testing of Stak Block wall systems. 


\section{Chapter $7 \quad$ Conclusions and}

\section{Recommendations}

\subsection{Introduction}

This report sought to investigate the feasibility of using Stak Blocks as a loadbearing and lateral force resisting structural system. The results from materials testing, full-scale shear wall testing, and computer modeling have shown that not only is it feasible to use Stak Blocks, but that this material might, in some ways, be superior to conventional wood framed construction.

The Stak Block was developed as an evolution or improvement of the straw bales used in construction. This study has shown that the product was successful in this endeavor as the blocks performed very well as a gravity and shear force resisting structural system. The available ductility supply and ability to withstand increasing forces after the point of first yield without approaching collapse or instability at very high drifts is a very positive characteristic displayed by the Stak Block wall systems. 


\subsection{Conclusions}

This section provides some of the more specific conclusions that were drawn from this study based on the testing of the Stak Blocks as a material, in the form of structural walls, and through analytical modeling.

\subsubsection{Materials Testing Conclusions}

Conclusions drawn from material testing:

- Stak Blocks have an elastic modulus of approximately 860 psi and a proportional (yield) strength limit of 23 psi (or 3,300 plf of wall).

- Stak Blocks’ elastic moduli are twelve times stiffer than those seen in straw bales. Additionally, at a similar strain value of $10 \%$, the straw blocks withstood stresses six times greater than a conventional straw bale.

- The Stak Blocks exhibited satisfactory behavior when subjected to cyclic loading with the initial settlement "toe” region absent from secondary loadings.

- Modifications to the blocks, including sawing in half and removing the top portion, caused a slight $(\sim 10 \%)$ reduction in strength and stiffness.

- Following an initial long-term loading, block relaxation is not as significant.

- Blocks are influenced by changes in temperature and humidity.

- For prestressing, $1 / 2$ inch thread bar with $55 \mathrm{ksi}$ yield strength is sufficient for the loads anticipated in construction. An equivalent $5 / 8$ inch thread bar would only need to be Grade 36 .

- A loop of wire rope of $1 / 4$ inch diameter is much less stiff and has a failure load of just over one half that of $1 / 2$ inch thread bar. 
- Longer (3 inch) screws should be used to attach sheathing to Stak Blocks and have a yield strength of 65 lbs/fastener (versus 186 lbs/fastener for 8d nails into wood).

- The block-to-block shear interface strength provided by interlocking studs is entirely dependent on normal prestressing forces.

- Inter-block sliding controls wall shear strength instead of mechanical fasteners to transfer shear to top and sill plates.

\subsubsection{Structural Wall Construction Conclusions}

Conclusions drawn from construction of walls:

- Out-of-plane temporary shoring, as well as tamping to ensure snug block placement, are both recommended during erection of Stak Block walls.

- To avoid Building Code visual inspection issues with coupling nuts, drill and epoxy short lengths (3 to 4 feet) of thread bar into foundation and provide inspection holes in course of blocks where prestressing bars will splice.

- Use 11 7/8 inch LSL double top plate to provide even distribution of prestressing forces.

- Provide shear transfer between top and sill plates and straw blocks with Simpson ${ }^{\circledR}$ mending plates or nails driven into straw material.

- If possible, use screws with the full shaft threaded to the head to allow for the sheathing to be pulled snug to the surface of the straw and avoid stripping.

- Wire rope falls slack and provides numerous issues with installation and applying appropriate prestressing forces. 
- Because of irregularities from block construction, Stak Block walls will tend to “lean” out-of-plane until adjacent floor or roof framing is installed.

\subsubsection{Structural Wall Testing Conclusions}

Conclusions drawn from full-scale wall testing:

- Blocks do not experience stresses past their proportional limit because of prestressed system performance.

- Bare, unsheathed wall specimen performed well and despite diagonal shear failure, withstood increasing load up to $6.75 \%$.

- Wire rope prestressing does not perform nearly as well as thread bar due to decreased stiffness and strength. Wall 3 with sheathing on one side and wire rope prestressing only performed slightly better than the unsheathed, Wall 1 with thread bar.

- Elongation was an issue and re-tensioning of tightening nuts may become necessary following large shear forces.

- Gaps formed between panel edges; staggering OSB edges and block edges or more rigid attachment would help to prevent this.

- Nails spaced at 4 inches as well as Simpson ${ }^{\circledR}$ Mending Plates spaced at 24 inches both provided sufficient shear transfer and allowed practically zero slippage between the blocks and adjacent wood top or sill plates.

- Relaxation was less of an issue on a global wall scale than shifts in the wall that caused drastic drops in prestressing forces. 
- Considerable Hysteretic Energy was dissipated due to the fact that yielding of the walls occurred when blocks begin to slide instead of the irreversible structural damage to panels and fasteners seen in wood framed shear walls.

- The ability of the prestressed Stak Block walls to absorb and dissipate energy without incurring substantial damage or imminent collapse at drift levels well past a yield point is very important in areas of high seismicity.

- None of the four wall specimens experienced failure or collapse, and in fact exhibited sustained post-yield stiffness, at drift levels as high as 6.75\%.

- Wall 2, with sheathing on both sides, performed as well, if not better than similarly constructed and tested wood framed walls with sheathing on one side.

- Sheathing on one side increased wall yield strength by approximately $90 \%$, as well as the elastic and post-yield stiffnesses 75\%.

- All walls exhibited ductility capacities, $\delta_{c} / \delta_{y}$, between 12 and 16 and strainhardening ratios, $\alpha_{s}$, of 0.14 to 0.20 . Prior studies of wood framed walls have shown values of 8.7 and 0.046 respectively for these two ductility measures.

- Reloading stiffness values ranged from 35 to $44 \%$ of post-yield backbone values. Reloading of wood framed specimens typically is much softer than the post-yield backbone.

- Stak Block walls exhibited Immediate Occupancy and Life Safety limit states over two times larger than those seen in timber framed shear walls. 


\subsection{Recommendations for Future Work}

It is recommended that further investigations into the long-term creep or relaxation of Stak Block wall systems be performed. Also, shear wall testing with higher prestressing loads could be conducted as well as displacing to very high drift levels (10 to $15 \%)$ to force collapse or failure of the wall system for observation purposes.

Additionally, other prospective uses of the Stak Blocks in construction may need to be explored as more ideas for their use come about. These may include, but are not limited to: filling of the cylindrical voids with reinforced concrete, the use of other rapidly renewable materials in conjunction with the blocks such as bamboo prestressing, and outof-plane lateral loading in sound, or retaining, wall applications. Further investigations into the types of construction outlined in this report focusing on issues such as inter-block relative sliding and block compressive strains during testing could also prove useful. Furthermore, the development of models to evaluate the dynamic behavior of the Stak Block wall system will help to determine appropriate design shear forces to be used in industry.

At this time, the performance of the prestressed Stak Block system is adequate enough to move forward with further development and standardization of construction details and specifications. While code-acceptance of this brand new building material is still dependent on future research and a further understanding of this relatively complex structural system, it is not unreasonable to foresee one- and two-story structures made of Stak Blocks in the next decade. The fact that an agricultural byproduct such as rice straw that was burned in California just a decade ago can be utilized in new construction is very encouraging. Straw is a rapidly renewable, locally sourced product that is easily turned 
into a viable building material that exhibits encouraging ductility and load-bearing

behavior. Stak Block’s performance is comparable, and sometimes superior, to conventional timber stud framed shear walls and shows that straw-derived, composite materials have a future in building technology. 


\section{References}

Amada, Shigeyasu, and Untao, Sun (2001). "Fracture Properties of Bamboo.”

Composites: Part B, Vol. 32, pp. 451-459.

American Forest and Paper Association (2005). American Wood Council. National Design Specifications for Wood Construction - ASD/LRFD. Washington D.C.

American Society of Civil Engineers (2000). FEMA 356: Prestandard and Commentary for the Seismic Rehabilitation of Buildings United States, Federal Emergency Management Agency. Washington D.C.

American Society of Civil Engineers (2007). United States. ASCE/SEI 41/06: Seismic Rehabilitation of Existing Buildings. Reston, VA.

American Society of Civil Engineers (2006). United States. ASCE/SEI 7-05: Minimum Design Loads for Buildings and Other Structures. Reston, VA.

Applied Technology Council (2005). United States, Federal Emergency Management Agency. FEMA 440: Improvement of Nonlinear Static Seismic Analysis Procedures. Washington D.C.

Ash, Cale; Aschheim, Mark; and Mar, David (2003). "In-Plane Cyclic Tests of Plastered Straw Bale Wall Assemblies.” Ecological Building Network. Oct. EBNet, Website Accessed October 20, 2008.

$<$ http://www.ecobuildnetwork.org/pdfs/InPlane_Wall_Tests_Small.pdf>

Bentley Systems (2009). RAM Advanse v. 9.0, Computer Software. Bentley Systems. 
Blum, Brandice (2002). “Load Carrying Behavior of On Edge Straw Bale Walls,” MS thesis, University of Manitoba at Winnepeg, Manitoba, Canada.

Bou-Ali, Ghailene (1993). “Straw Bales and Straw Bale Wall Systems,” MS thesis, University of Arizona at Tuscon, AZ.

Breyer, Donald E., Kenneth J. Fridley, David G. Pollock Jr., Kelly E. Cobeen (2003). Design of Wood Structures - ASD. New York: McGraw Hill.

Brink, Francis E. and Rush, Paul J. (1966). “Bamboo Reinforced Concrete Construction,” February, U.S. Naval Civil Engineering Laboratory, Port Hueneme, CA.

California Rice Commission (2009). "Recycling of Straw in the Field." California Rice Commission - Recycling, Website Accessed October 15, 2009. <http://www.calrice.org/a5a_recycling.htm>

Carrasco, Andrea; Fronda, John; and MacRae, Brian (2002). "Mechanical Properties of Bamboo,” October, Undergraduate Presentation, University of Southern California, Los Angeles, CA.

Centre for Building Performance Research (2003). "Embodied Energy Coefficients." Victoria University of Wellington, New Zealand, Website Accessed November 10, 2009. <http://www.victoria.ac.nz/cbpr/documents/pdfs/ee-coefficients.pdf>.

Chiras, Daniel D. (2000). The Natural House. White River Junction, VT, USA: Chelsea Green Publishing Company.

Christian, Jeff (1998). “Steady State Thermal Conductance of Plastered StrawBale Walls”, Oak Ridge National Laboratories, Oak Ridge, Tennessee.

Dodge, Darius (2008). "Investigation of Seismic Performance of Timber Shear Walls Utilizing Spray Applied Polyurethane Foam,” MS thesis, California Polytechnic State University at San Luis Obispo, CA.

Elwood, Kenneth J.; Matamoros, Adolfo B.; Wallace, John W.; Lehman, Dawn E.; Heintz, Jon A.; Mitchell, Andrew D.; Moore, Mark A.; Valley, Michael T.; Lowes, Laura N.; Comartin, Craig D.; and Moehle, Jack P. (2007). "Update to ASCE/SEI 41 Concrete Provisions." Earthquake Spectra, Vol. 23, pp. 493-523.

Faine, Michael, and Zhang, John (2002). “A Pilot Study Examining the Strength, Compressibility and Serviceability of Rendered Straw Bale Walls for Two Storey Load Bearing Construction,” University of Western Sydney at Sydney, NSW, Australia. 
Field, Kristin; Woods, James; and Fedrigo, Claudia (2005). "Structural Testing of Straw Bales in Axial Compression," Undergraduate Presentation, University of Colorado at Boulder, CO.

Gatto, Kip, and Uang, Chia-Ming (2003). "Effects of Loading Protocol on the Cyclic Response of Woodframe Shearwalls.” Journal of Structural Engineering, Vol. 129, No. 10, pp. 1384-1393.

Heredia, Horacio (2010). “Cyclic Testing of Conventional Woodframe Shear Walls -Influence of Non-Structural Finish Material,” MS thesis, California Polytechnic State University at San Luis Obispo, CA.

Ibarra, Luis F.; Medina, Ricardo A.; and Krawinkler, Helmut (2005). "Hysteretic Models that Incorporate Strength and Stiffness Deterioration." Earthquake Engineering and Structural Dynamics, Vol. 34, No. 12, pp. 1489-1511.

International Code Council (2006). International Building Code 2006. Country Club Hills, IL.

Intertek (2007b). "2-Hr Fire Resistance Test of a Non-Loadbearing Wheat Straw Bale Wall,” Project No. 3098054A, Intertek Testing Services, Elmendorf, Texas, Research Report, Prepared for the Ecological Building Network, Sausalito, CA.

Jain, Seema; Kumar, Rakesh; and Jindal, U.C. (1992). "Mechanical Behaviour of Bamboo and Bamboo Composite.” Journal of Materials Science, Vol. 27, pp. 4598-4604.

Jenkins, Bryan M. (1991). "Survey Documents Open Burning in San Joaquin Valley." California Agriculture Magazine July-August, pp. 12-16

King, Bruce (2003). "Load-Bearing Straw Bale Construction." Ecological Building Network. June 30. EBNet, Website Accessed October 15, 2009. <http://www.ecobuildnetwork.org/pdfs/LoadBearing_SB_Const.pdf $>$.

King, Bruce (1998). "Straw-Bale Construction." Building Standards. September-October. pp. 18-24.

Korman, Benjamin Z. (2005) "Culm Blocks." U.S. Patent 6,951,080 B2. October 4.

Krawinkler, Helmut; Parisi, Francisco; Ibarra, Luis; Ayoub, Ashraf; and Medina, Ricardo (2001). Development of a Testing Protocol for Woodframe Structures. The CUREE Caltech Woodframe Project. 
Krawinkler, Helmut; Zarein, Farzin; Ibarra, Luis; Medina, Ricardo; and Lee, Sueng-Je (2003). Seismic Demands for Single and Multi-Story Wood Buildings. The CUREE Caltech Woodframe Project.

McCabe, Joseph (1994). “The Thermal Resistivity of Straw Bales for Construction,” MS thesis, University of Arizona at Tuscon, AZ.

National Science Foundation (2009). "Standing Strong - 2009 NEESWood Capstone Test.” Network for Earthquake Engineering Simulation. Dec. NSF, Website Accessed December 9, 2009.

$<$ http://www.nsf.gov/news/newsmedia/neeswood/index2.jsp>

Nirman, Luit (2009). "Bamboo as Viable Structural Material." Luit Nirman, Website Accessed November 10, 2009.

$<$ http://www.assambambooworld.com/bamboo-structuralmaterial.htm $>$.

Platts, Bob, and Chapman, Linda (1996). "Developing and Proof-Testing the 'Prestressed Nebraska’ Method for Improved Production of Baled Fibre Housing,” Canada Mortgage and Housing Corporation.

Rahnama, Moshen, and Krawinkler, Helmut (1993). "Effects of Soft Soils and Hysteresis Model on Seismic Demands,” Report No. 108, The John A. Blume Earthquake Engineering Center, Department of Civil and Environmental Engineering, Stanford University, Stanford, CA.

Reiner, Mark; Pitterle, Mark; and Whitaker, Michael (2007). "Embodied Energy Considerations in Existing LEED Credits." September, Symbiotic Engineering, Website Accessed November 10, 2009. $<$ http://www.symbioticengineering.com/includes/content/publications/embodied_energy_consi derations_in_existing_leed_credits.pdf $>$.

Ruppert, Jeff and Matt Grandsaert (1999). “A Compression Test of Plastered Straw-Bale Walls,” University of Colorado at Boulder, CO.

State of California (1991). Heath and Safety Code, § 41865. Connelly-AreiasChandler Rice Straw Burning Reduction Act.

Stephens, Don and Budinger \& Associates, Inc. (2000) Laboratory Test. Spokane, WA. July 13.

Stone, Nehemiah (2003). "Thermal Performance of Straw Bale Wall Systems." Ecological Building Network. Oct. EBNet, Website Accessed October 15, 2009. <http://www.ecobuildnetwork.org/pdfs/Thermal_properties.pdf>. 
United States Department of Agriculture (2008). "Statistics by Subject - Crops \& Plants - Field Crops - Rice.” National Agricultural Statistics Service. April. NAAS, Website Accessed April 30, 2009.

$<$ http://www.nass.usda.gov/QuickStats/index2.jsp>

United States Green Building Council (2005). LEED-NC: For New

Construction, Reference Guide Version 2.2. Washington D.C.

Vardy, Stephen P. (2009). "Structural Behaviour of Plastered Straw Bale Assemblies under Concentric and Eccentric Loading,” Dissertation. Queen’s University Kingston, Ontario, Canada.

Watts, K. Chris; Wilkie, Ken; Thompson, Kim; and Corson, Jennifer (1995). "Thermal and Mechanical Properties of Straw Bales As They Relate To a Straw House." Rep. No. 95-209, Canadian Society for Agricultural Engineering, Ottawa, Ontario.

Wilson, Alex (1995). "Straw: The Next Great Building Material?" Environmental Building News, May 1.

Zhang, John (2000). "Load-Carrying Characteristics of a Single Straw Bale Under Compression,” University of Western Sydney at Sydney, NSW, Australia, July. 